\title{
ORIENTATION OF CRYSTALLINE OVERLAYERS ON AMORPHOUS SUBSTRATES BY ARTIFICIALLY PRODUCED SURFACE RELIEF STRUCTURES
}

\author{
By \\ Dale Clifton Elanders \\ B.S., Massachusetts Institute of Technology \\ (1974) \\ M.S., Massachusetts Institute of Technology \\ (1974) \\ SUBMITTED IN PARTIAL FULFILLMENT \\ OF THE REQUIREMENTS FOR THE \\ DEGREE OF \\ DOCTOR OF PHILOSOPHY \\ at the \\ MASSACHUSETTS INSTITUTE OF TECHNOLOGY \\ (January 1978)
}

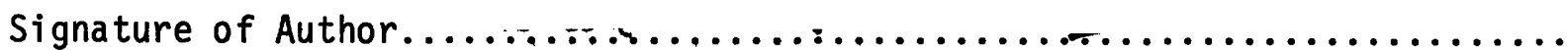 Department of Electrical Engineering and Computer Science, January 20, 1978

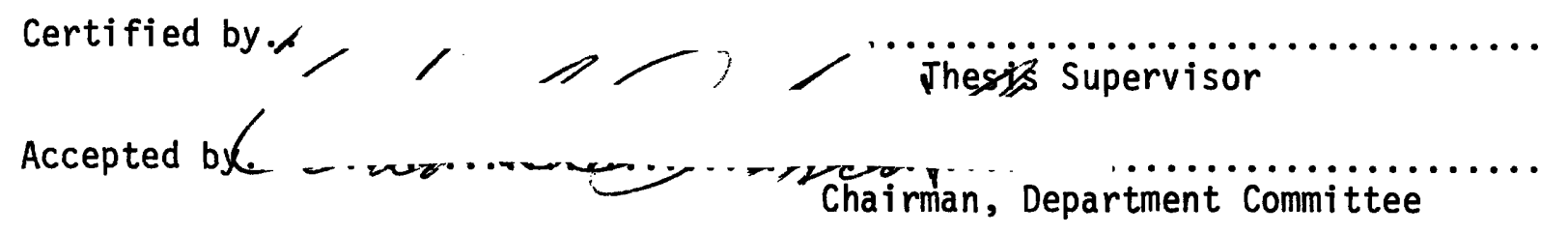

$$
\begin{gathered}
\text { ARO IIES } \\
\text { MASSACHUSETS INSTITUTE } \\
\text { OF TECHNOLOGY } \\
\text { JUL } 2 \% 1978 \\
\text { LIBRARIES }
\end{gathered}
$$




\section{CONTENTS}

ABSTRACT $\quad 5$

$\begin{array}{ll}\text { LIST OF FIGURES } & 7\end{array}$

LIST OF SYMBOLS 13

$\begin{array}{ll}\text { ACKNOWLEDGEMENTS } & 17\end{array}$

$\begin{array}{ll}\text { I. INTRODUCTION } & 19\end{array}$

II. OVERLAYER ORIENTATION EFFECTS INDUCED BY SURFACE RELIEF 28 STRUCTURES ON AMORPHOUS SUBSTRATES

2.1 Introduction 28

2.2 Anisotropic Interfacial Tensions 29

2.3 Generalized Interfacial Tensions for Surface Relief 34 Structures

2.4 Orientation Effects at Equilibrium 39

2.5 The Choice of Overlayer-Substrate Combinations and 42 Substrate Surface Relief Structure for an Experimental Demonstration of the Orientation Effect

III. FABRICATION OF SURFACE RELIEF STRUCTURES

$\begin{array}{lll}3.1 & \text { Introduction } & 47\end{array}$

3.2 Mask Pattern Generation by Holographic Lithography 50

3.3 Pattern Replication by X-ray Lithography 57

3.3.1 Process Capabilities 57

3.3.2 Considerations in the Choice of $\mathrm{Cu}_{\mathrm{L}}$ and $\mathrm{C}_{\mathrm{k}} \quad 58$ $X$-rays for Submicrometer Pattern Replication

3.3.3 The X-ray Source 66

3.3.4 The Fabrication of X-ray Masks 71

3.3.5 The Maintenance of Intimate Mask-Substrate 79 Contact 

3.3.6 Experimental Results - PMMA Profiles obtained Using $\mathrm{Cu}_{L}$ and $\mathrm{C}_{k} X$-rays

3.4 Etching of the Surface Relief Structures 89

3.4.1 Ion-Beam Etching - Redeposition 90

3.4.2 Reactive-Ion-Etching 93

3.4.3 Final Cleaning 99

3.5 Characterization of Surface Relief Structures 100

3.6 Conclusion 103

IV. ORIENTATION OF LIQUID CRYSTALS BY ARTIFICIAL SURFACE RELIEF 104 STRUCTURES

$\begin{array}{lll}4.1 & \text { Introduction } & 104\end{array}$

4.2 Properties of Liquid Crystals 105

4.3 The Generalized Interfacial Tension of a Liquid 112 Crystal and a Surface-Relief Structure

4.4 The Orientation of Liquid Crystals by Square-Wave 115 Gratings on Amorphous $\mathrm{SiO}_{2}$ Substrates - Experiment

4.4.1 The Grating Structures 117

4.4.2 Alignment of MBBA in the Nematic Phase 118

4.4.3 Alignment of M-24 in the Nematic and Smectic 123 A Phases

4.5 Conclusion

125

V. ORIENTATION OF SOLID CRYSTALLINE OVERLAYERS BY SURFACE RELIEF STRUCTURES ON AMORPHOUS SUBSTRATES

5.1 Introduction

5.2 General Features of Thin Film Growth 127

5.2.1 The Thin Film Growth Process 127

5.2.2 Equilibrium Form of a Crystalline Island on an 134 Amorphous Substrate 
5.2.3 Nucleation

5.2.4 Decoration

5.2.5 Is land Growth

5.2.6 Coalescence and Network Formation

5.2.7 Fiber Textures on Smooth Amorphous Substrates

5.3 A Model for Orientation of Thin Film Deposits on Amorphous Substrates by Square-Wave Surface-Relief Gratings

5.3.1 Surface-Relief Structure Characterization

5.3.2 Nucleation and Growth on a Square-Wave

5.4 Deposition of Crystalline Overlayers on Square-Wave Gratings in Amorphous $\mathrm{SiO}_{2}$ - Experiment

5.4.1 Experimental Procedure

5.4.2 Decoration Experiments

5.4.3 Potassium Chloride Deposition Experiments

5.4.4 Tin Deposition

VI. CONCLUSION

6.1 Summary of Work 207

6.2 Proposals for Future Work 209

APPENDIX A Anisotropic Etching of Silicon $\quad 215$

APPENDIX B Polyimide X-ray Mask Fabrication Procedure 220

APPENDIX C Preparation and Spinning of Polymethly Methacrylate 229 (PMMA)

APPENDIX D Properties of MBBA and M-24 


\title{
ORIENTATION OF CRYSTALLINE OVERLAYERS ON AMORPHOUS SUBSTRATES BY ARTIFICIALLY PRODUCED SURFACE RELIEF STRUCTURES
}

\author{
By \\ Dale Clifton Flanders \\ Submitted to the \\ Department of Electrical Engineering and Computer Science \\ on January 20, 1978 in partial fulfillment of the \\ requirements for the Degree of Doctor of Philosophy
}

\begin{abstract}
Overlayers of crystalline materials on smooth amorphous substrates tend to be more or less random polycrystalline. The absence of longrange order in the amorphous substrate is reflected in the absence of long-range order in the overlayer. The new concept investigated in this work is that a single crystal film can be produced on an amorphous substrate by introducing an artificial surface relief structure having long range order. A simple thermodynamic argument indicates that these surface relief structures need not have dimensions of the order of the lattice parameter of the crystalline overlayer but rather structures can be used whose dimensions are comparable to the size of the naturally occuring single crystal grains of polycrystalline films. It is argued that at equilibrium an overlayer material which exhibits an anisotropic interfacial tension (this includes the liquid crystal mesophases as well as solid crystals) will adopt a unique single-crystal orientation with respect to a suitable surface relief structure on an amorphous substrate. For example, it is shown that at equilibrium a cubic material whose $\{100\}$ planes have minimum interfacial tension will be oriented with $\{100\}$ parallel to a substrate, and a $\langle 100\rangle$ direction parallel to the groove direction of a square-wave grating on the substrate.

It was found that the major problem in experimentally demonstrating the predicted orientation effects was the fabrication of the required surface relief structure. New very soft $X$-ray $1 i$ thographic and reactiveion-etching fabrication techniques were developed. With these techniques $160 \mathrm{~nm}$ linewidth square-wave gratings having smooth vertical sidewalls and sharp corners with less than $5 \mathrm{~nm}$ curvature were fabricated in amorphous silicon dioxide.
\end{abstract}

A model of nematic and smectic A liquid crystals indicates that simple square-wave structures should induce uniform "single crystal" orientation of these materials. Experiments were performed using the 1 iquid crystals MBBA and M-24. As expected, uniform orientation was induced in MBBA in the nematic phase and in M-24 in the nematic and 
smectic A phases.

A detailed model of the (nonequilibrium) thin film growth process showed that under certain deposition conditions a surface relief structure could induce a solid crystalline deposit to acquire the single crystal orientation predicted by the equilibrium interfacial tension model. Experiments were performed using square-wave grating structures on amorphous $\mathrm{SiO}_{2}$ substrates. Depositions of potassium chloride from aqueous solution and tin by vacuum evaporation were done on these structures. Potassium chloride crystallites were oriented with $\{100\}$ parallel to the substrate and $\langle 100\rangle$ parallel to the groove direction as predicted by the thin film growth model. The orientation effect was not observed on structures whose square profile had been rounded. This is explained qualitatively by the model of thin film growth. A series of tin depositions on square-wave gratings yielded results consistent with the model of thin film growth, but a strong orientation effect was not observed. Only weakly preferred orientation seems to have been induced by the surface relief structure. It is concluded that smaller periodicity grating structures with sharper edges and corners will be required to induce a strong orientation effect with tin.

A new method of orienting crystalline (anisotropic) overlayers on an amorphous substrate by surface relief structures on the substrate has been analyzed and demonstrated. New submicrometer fabrication techniques had to be developed in order to demonstrate the orientation effect. These techniques may have broad application in the fields of microelectronics and integrated optics as well as in the work presented here. It is believed that the models and demonstrations of overlayer orientation presented here are but a first step in what will be an exciting new field of investigation. In essence, a new degree of freedom has been introduced in the science and technology of surfaces and thin film growth.

Thesis Supervisor: Dr. Henry I. Smith

Title: Adjunct Professor of Electrical Engineering and Computer Science and Assistant Group Leader at M.I.T. Lincoln Laboratory. 


\section{LIST OF FIGURES}

FIGURE

PAGE

1-1. A schematic illustration of the equilibrium orientations of a cubic material on an amorphous substrate with a surface structure.

2-1. Cross-sections of representative interfacial tension plots are shown for a conventional isotropic liquid, a liquid crystal and a cubic crystalline solid.

2-2. Schematic cross-sections are shown of a square-wave interface and a sawtooth interface between an amorphous and a crystalline material.

2-3. A polar plot is shown of the generalized interfacial tension, $\sigma_{q}$, of the square-wave interface depicted in Fig. 2-2(a).

3-1. A schematic illustration is shown of the process developed for fabricating submicrometer-period square-wave-grating surfacerelief structures in amorphous $\mathrm{SiO}_{2}$.

3-2. A schematic illustration is shown of the configuration of optical components used to expose gratings in Shipley AZ-1350 photoresist by interfering two laser beams.

3-3. An SEM micrograph of a holographic exposure of AZ-1350 photoresist on a $100 \mathrm{~nm}$ thick gold film is shown.

3-4. An SEM micrograph of a $320 \mathrm{~nm}(3200 \AA)$ period grating of Shipley AZ-1350J photoresist on a glass substrate produced using simultaneous-exposure-and-development holographic lithography.

3-5. Illustrates the $X$-ray lithographic replication process using an electron bombardment $X$-ray source.

3-6. Differential dissolution rate of an $\sim 0.3 \mu \mathrm{m}$ thick film of PMMA as a function of energy dissipated in the polymer for three different solvents: ethyl alcohol, a solution of $40 \%$ (MIK) and $60 \%$ (IPA), and pure MIK.

3-7. The calculated maximum range of photo electrons in PMMA

3-8. A plot of the attenuation $(\mathrm{db} / \mu \mathrm{m})$ of gold and polyimide versus wavelength for soft $X$-rays (1-100 $\AA$ ). 
3-9. A schematic diagram of the apparatus used to implement

$X$-ray lithography with $\mathrm{Cu}_{L}$ and $C_{k}$ X-rays.

3-10. The calculated $X$-ray power from a copper $X$-ray source, operating at $-8 \mathrm{kv}$, absorbed in 1 um of PMMA.

3-11. The processing sequence for fabricating polyimide membrane

$X$-ray masks with gold absorber patterns.

3-12. a). A schematic cross-section is shown of the bonding of a copper tube etching holder to a polyimide film prior to etching of the glass substrate.

b). The method of removing the glass substrate by etching in dilute hydroflouric acid is illustrated.

3-13. Schematic cross-section of a polyimide $X$-ray mask developed for high resolution $X$-ray lithography using the $\mathrm{Cu}_{L}$ and $C_{k} X$-ray wavelengths.

3-14. Cross-sections of two types of polyimide $X$-ray masks are shown where the gold absorber pattern in on the "back" of the mask.

3-15. Schematic cross-sections are shown of three mask and substrate geometries used to obtain intimate contact between a mask and substrate using electrostatic pressure.

3-16. SEM micrograph of a $320 \mathrm{~nm}(3200 \AA)$ period grating of PMMA on an amorphous $\mathrm{SiO}_{2}$ substrate which was exposed using the $\mathrm{Cu}_{\mathrm{L}}(13.3 \AA) \mathrm{X}$-ray.

3-17. A higher magnification SEM micrograph is shown of the

PMMA structure shown in Fig. 3-16.

3-18. SEM micrograph of a $320 \mathrm{~nm}(3200 \AA)$ period grating of

PMMA on an amorphous $\mathrm{SiO}_{2}$ substrate exposed using the $\mathrm{Cu}_{\mathrm{L}}$ $X$-ray.

3-19. A higher magnification SEM micrograph is shown of the PMMA structure shown in Fig. 3-18.

3-20. An SEM micrograph is shown of a $320 \mathrm{~nm}(3200 \AA)$ period grating of PMMA on an amorphous $\mathrm{SiO}_{2}$ substrate exposed using the $C_{k}(44.8 \AA) X$-ray. 
3-21. SEM micrographs showing (Top) AZ-1350J photoresist pattern on

a silicon substrate prior to ion-beam etching, (Center) after ion beam etching to a depth of $130 \mathrm{~nm}$, (Bottom) after dissolution of photoresist in solvent and plasma ashing in oxygen.

3-22. Schematic diagram of the apparatus used to do reactive-ionetching.

3-23. Schematic of the sequence of steps in the 1iftoff process.

3-24. This SEM micrograph shows a $320 \mathrm{~nm}(3200 \AA)$ period surface relief structure in amorphous $\mathrm{SiO}_{2}$. The structure was fabricated by reactive-ion-etching with $\mathrm{CHF}_{3}{ }^{2}$ gas through a $20 \mathrm{~nm}$ thick chromium mask.

3-25. A higher magnification SEM micrograph is shown of the amorphous 98 $\mathrm{SiO}_{2}$ surface relief structure shown in Fig. 3-24.

3-26. TEM micrographs are shown of a square-wave-grating surfacerelief structure in amorphous $\mathrm{SiO}_{2}$.

102

4-1. Schematic representations of the isotropic, nematic, smectic $A$ and crystalline phases of a liquid crystal material.

4-2. The possible equilibrium orientations of a nematic (or smectic) liquid crystal confined between smooth parallel interfaces.

4-3. Schematic illustration of possible configurations of a nematic liquid crystal at a square-wave grating interface.

4-4. A schematic cross-section and top view are shown of the "sand- 116 wich" assembly used to investigate surface-rel ief structure orientation effects in nematic and smectic $A$ liquid crystals. 4-5. An optical micrograph is shown of a texture of dis-
clinations of a nematic liquid crystal on a smooth amorphous substrate.

5-1. A schematic illustration is shown of the distinct stages of 128 thin film growth.

5-2. Transmission-electron-micrograph of a gold deposit on rocksalt at very low average film thickness.

5-3. An illustration of the application of the Gibbs-Wulff construction to the determination of the equilibrium form of a cubic deposit on a substrate. 
FIGURE

PAGE

5-4. A schematic representation of the equilibrium forms of the possible interfacial tensions as enumerated in Table 2-1.

5-5. The diagrams show cross-sections, as determined by the GibbsWulff construction, of the equilibrium form of a cubic crystalline material and an isotropic material in a $90^{\circ}$ corner of an isotropic material.

5-6. The equilibrium forms of a cubic deposit at a step and on a plane are shown.

5-7. A plot is shown of the calculated ratio of nucleation rate at a step, $I_{s}$, and on a flat plane, $I_{f}$, versus nuclei contact angle $\theta_{c}$ for an isotropic material.

5-8. A plot is shown of the calculated ratio of nucleation rates at a step and on a plane for a cubic material.

5-9. A transmissionmelectron-micrograph is shown of a tin deposition on $\mathrm{NaCl}$.

5-10. The coalescence and recrystallization phenomenon for two islands is shown.

5-11. Illustrations are shown of the coalescence of a group of islands for the cases of rapid and slow coalescence.

5-12. An idealized representation is shown of the square-wave surface-relief structure used for overlayer orientation experiments.

5-13. A schematic representation is shown of a grid-type squarewave structure which could be used to delay the onset of the network stage of $f i l m$ growth and induce overlayer orientation.

5-14. The sequence of growth of a cubic material on a squarewave structure is shown for the case of perfect decoration of vertical steps.

5-15. The sequence of growth of a cubic material on a square-wave structure is shown where nuclei occur both at the steps and between the steps.

5-16. The sequence of growth of a cubic material on a square-wave structure is shown where the nucleation is random and few nuclei occur at the steps. 
5-17. The procedure used for investigating the effect of surface relief gratings on thin film growth is illustrated.

5-18. The procedure used for fabricating square-wave surface

5-19. A transmission-electron-micrograph is shown of gold islands on an amorphous $\mathrm{SiO}_{2}$ surface with a 1.5-nm-deep 320-nm-period squarewave surface-relief grating fabricated using ion-beam-etching.

5-20. A transmission-electron-micrograph is shown of a gold deposit of 179 $1.5 \mathrm{~nm}$ average thickness on a square-wave-grating surface-relief structure in $\mathrm{SiO}_{x}$ fabricated by liftoff of evaporated $\mathrm{SiO}_{2}$.

5-21. A transmission-electron-micrograph is shown of a gold deposit

on a 5-nm-deep, 320-nm-period square-wave-grating in amorphous $\mathrm{SiO}_{2}$ fabricated using reactive-ion-etching.

5-22. A transmission-electron-micrograph is shown of a gold deposit of $1.5 \mathrm{~nm}$ average thickness on an $\mathrm{SiO}_{2}$ substrate on which a $320 \mathrm{~nm}$ period grating of $10 \mathrm{~nm}$ thick carbon strips has been defined by the liftoff process.

5-23. A scanning-transmission-electron micrograph is shown of $\mathrm{KCl}$ crystallites grown on a $320 \mathrm{~nm}$ spatial-period square-wave surfacerelief grating in amorphous $\mathrm{SiO}_{2}$ showing that the grating has induced an oriented crystal growth where $\langle 100\rangle$ directions are parallel to the grating grooves.

5-24. An optical micrograph is shown of $\mathrm{KCl}$ crystallites grown on a smooth $\mathrm{SiO}_{2}$ surface.

5-25. Transmission-electron micrographs are shown of KCl crystallites 188 grown on a $320 \mathrm{~nm}$ spatial-period square-wave surface-relief grating in amorphous $\mathrm{SiO}_{2}$.

5-26. A plot is shown of the measured deviation of the $\langle 100\rangle$ direction of the $\mathrm{KCl}$ crystallites from the grating groove direction for the micrographs shown in Fig. 5-25.

5-27. An optical micrograph at $1000 \mathrm{X}$ is shown of a $\mathrm{KCl}$ deposition on a square-wave surface-relief structure in $\mathrm{SiO}_{2}$ with an irregular step spacing which in some areas is as large as $3 \mu \mathrm{m}$. 
FIGURE

PAGE

5-28. Micrographs are shown of $\mathrm{KCl}$ depositions on a square-wave

194 structure which has been degraded by isotropically etching $1.5 \mathrm{~nm}$ of $\mathrm{SiO}_{2}$ from the structure shown in Fig. 5-23.

5-29. A scanning-transmission-electron micrograph is shown of an $0.8 \mathrm{~nm}$ average thickness tin deposit on a $50-\mathrm{nm}$-deep $320 \mathrm{~nm}$ period square-wave grating in amorphous $\mathrm{SiO}_{2}$.

5-30. Scanning transmission-electron micrographs are shown of a $4 \mathrm{~nm}$ average thickness tin deposit on a 50-nm-deep, 320-nm-period square-wave grating in amorphous $\mathrm{SiO}_{2}$.

5-31. A transmission-electron micrograph is shown of a 40-nmaverage thickness tin deposit on a 50-nm-deep 320-nm-period square wave grating in amorphous $\mathrm{SiO}_{2}$.

5-32. A transmission electron micrograph is shown of an $80 \mathrm{~nm}$ average thickness tin deposit on a $50 \mathrm{~nm}$ deep $320 \mathrm{~nm}$ period square wave grating in amorphous $\mathrm{SiO}_{2}$.

5-33. A TEM micrograph of a 80-nm-average-thickness tin deposition 205 and the corresponding selected area electron diffraction pattern are shown.

6-1. Schematic representations are shown of two proposed methods for producing small areas of single crystal semiconductor for incegrated circuit devices.

6-2. A hexagonal-close-packed array of "spoke" surface-relief structures is shown. The molecular arrangement in a myelinic structure is shown schematically.

A-1. A schematic cross-section of a $\mathrm{Si}_{3} \mathrm{~N}_{4}-\mathrm{SiO}_{2}$ membrane $\mathrm{X}$-ray mask 216 A-2. A schematic diagram is shown of the sample holder used during anisotropic etching of silicon. 


\title{
LIST OF SYMBOLS
}

\author{
$\gamma \quad$ Interfacial tension \\ $\gamma(\vec{n}) \quad$ The interfacial tension of an interface where the orientation of \\ the interface is specified by the unit vector $\vec{n}$ \\ $(\vec{n}) \quad$ Unit vector specifying orientation of a crystal \\ dW Increment of work \\ $\mathrm{dA} \quad$ Increment of area \\ F Helmholtz free energy \\ E Internal energy \\ T Temperature \\ S Entropy \\ $f_{s} \quad$ Specific surface free energy or Helmholz free energy per unit area \\ $\mu_{i} \quad$ Chemical potential of the $i^{\text {th }}$ component of a system \\ $\Gamma_{i} \quad$ Interfacial excess of the $i^{\text {th }}$ component of a system \\ $\vec{r} \quad$ Radius vector of a polar plot \\ $I(x, y) \quad$ A periodic function of the cartesian coordinates $x$ and $y$ which \\ specifies the position of an interface. \\ $\sigma(\vec{n}) \quad$ Generalized interfacial tension \\ s Period of a surface releif structure or as a subscript denotes a \\ sawtooth structure, in Fig, 3-5 gives mask-substrate gap \\ $h \quad$ Height of a surface relief structure \\ q Subscript denoting a square wave interface \\ $\theta \quad$ The angle of deviation of a $\langle 100\rangle$ direction of a cubic material \\ from the $X$ direction or the half angle between two interfering \\ light beams
}




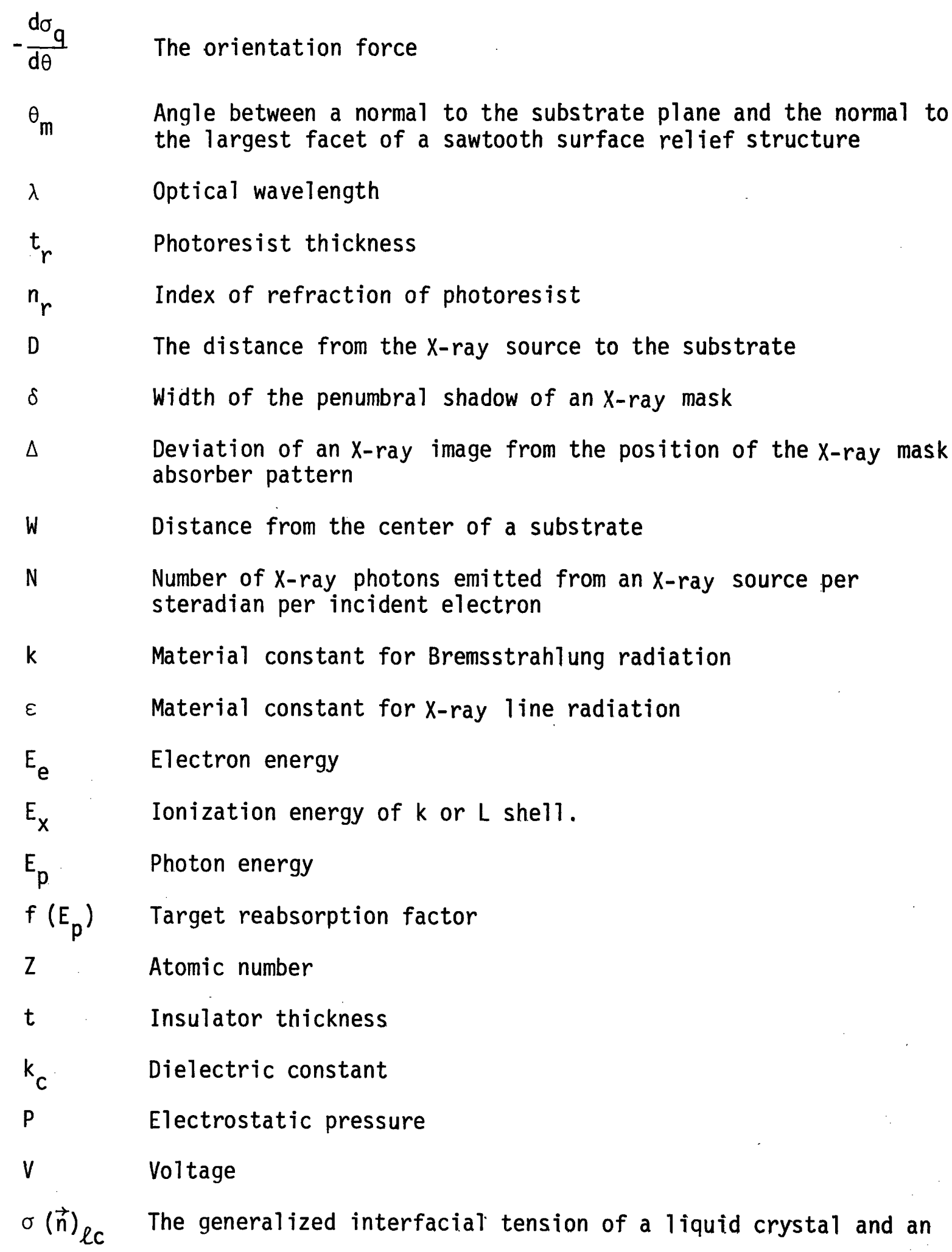


amorphous substrate

$F_{d} \quad$ Distortion free energy of a liquid crystal

$F_{d n} \quad$ Distortion free energy of a nematic liquid crystal

$F_{d s} \quad$ Distortion free energy of a smectic A liquid crystal

$\vec{k}$

Vector specifying the direction of propagation of a light wave

$\vec{D} \quad$ Vector specifying the direction of polarization of a light wave

sd Subscript denotes a substrate and deposit interface

$\mathrm{dv} \quad$ Subscript denotes a deposit and vapor or parent phase interface

sv Subscript denotes a substrate and vapor or deposit and parent phase interface

$\Delta G_{v} \quad$ (Gibbs) Free energy of formation per unit volume

K Boltzman's constant

$n_{1} \quad$ Adatom concentration

$n_{i e} \quad$ Adatom concentration at equilibrium

$\Delta G_{v t} \quad$ Density of Gibbs free energy of formation

$\gamma_{t} \quad$ Density of interfacial Gibbs free energy

$r \quad$ Nucleus size

$r^{*} \quad$ Critical nucleus size

$\Delta G_{f} \quad$ Gibbs free energy of formation

$\Delta G_{f} * \quad$ Critical Gibbs free energy of formation

$\mathrm{J} \quad$ Nucleation rate

$n_{0} \quad$ Number of nucleation sites per unit area

A Adatom impingement frequency

$\Delta g^{*} \quad$ Critical free energy of formation difference for two different nuclei 


$\begin{array}{ll}I_{f} & \text { Nucleation rate on a flat substrate } \\ I_{s} & \text { Nucleation rate at a step for an isotropic deposit } \\ \theta_{c} & \text { Contact angle between an isotropic deposit island and a substrate } \\ d & \begin{array}{l}\text { The small dimension of a cubic island on a substrate. X-ray source } \\ \text { size in Fig. } 3-5\end{array} \\ l & \text { The large dimension of a cubic island on a substrate } \\ w_{t} & \text { Width of the top of a square wave grating } \\ w_{g} & \text { Width of the groove of a square wave grating } \\ R_{t} & \text { Radius of the top edge of a square wave grating } \\ R_{g} & \text { Radius of the groove corner of a square wave grating } \\ i & \text { Subscript denotes an isotropic material } \\ c & \text { Subscript denotes a cubic material } \\ n_{s} & \text { Number density of nucleation sites at a step } \\ n_{f} & \text { Number density of nucleation sites on a flat substrate }\end{array}$




\section{ACKNOWLEDGMENTS}

The idea of using surface relief structures to manipulate thin film growth was originated by Dr. Henry I. Smith several years ago. During this work conducted at M.I.T. Lincoln Laboratory Dr. Smith has provided me with a great deal of guidance both personal and professional, for this I am deeply grateful.

Many members of Groups 86 and 87 at Lincoln Laboratory have contributed to this work. In particular I would like to thank Peter DeGraff for his expert assistance in microscopy and X-ray lithography, William Brogan and Richard Slattery for their assistance in deposition experiments, Muriel Dalomba, for her help in X-ray mask fabrication, and Pauline Phinney and Nick Efremow for their aid in sample preparation.

David Shaver has provided valuable ideas and assistance during this research. In particular I thank him for his work in automating the $X$-ray exposure process and in several facets of the liquid crystal experiments .

I am grateful to Ronald Pindak and Robert Myers, formerly of Harvard University, and Ralph Shaetzing of M.I.T. who contributed their knowledge and expertise in liquid crystals.

I wish to thank Professor John Vander Sande for his guidance and assistance with electron microscopy and Dr. Ernest Hall for his help in obtaining scanning transmission electron micrographs of several samples. 
I am deeply indebted to Dr. Hans Lehmann of R.C.A. Laboratory in Zurich Switzerland for his generous assistance in transferring the reactive-ion-etching technology to us.

I am also indebted to Stephen Bernacki who transferred to me his knowledge of X-ray lithography.

I wish to express my appreciation to Donna Mancuso and Deborah Pepe for their typing of the manuscript.

Finally I want to thank my wife Julia for her help in initial preparation of the manuscript and for her love and understanding which have helped to make this work pleasurable. 


\section{INTRODUCTION}

Single crystal thin films are of great importance in both basic research and for device applications. It is widely known that some single crystal films can be grown on smooth crystalline substrates. When a single crystal film of a material is grown on a substrate of the same material the process is known as homoepitaxy. If the film and substrate materials are different the phenomenon of single crystal film growth is known as heteroepitaxy. Homoepitaxy and heteroepitaxy are known to occur only on single crystal substrates ${ }^{1,2}$. Such substrates often tend to be relatively expensive and difficult to obtain in large sizes. Amorphous materials are much less expensive and large substrates are easily prepared. Therefore, the ability to produce single crystal films on amorphous substrates would be of great importance. However, deposition of crystalline materials on smooth amorphous substrates yields more or less randomly oriented polycrystalline films ${ }^{1,2,3}$. The failure to obtain single crystal films on smooth amorphous substrates is not surprising if one views the phenomenon of epitaxy as an ordering of the deposited overlayer induced by the "template" of the substrate. The local and long-range order of a crystalline substrate is reflected in the order of the overlayer. In the case of overlayers on smooth amorphous substrates the absence of long-range order in the substrate is reflected in the absence of long-range order in the overlayer.

The new concept demonstrated in this work is that a single crystal film can be grown on an amorphous substrate by introducing an 
artificially produced surface relief structure on the amorphous substrate. Furthermore, these surface relief structures need not have dimensions of the order of the size of the lattice parameter of the crystalline overlayer. Structures can be used whose dimensions are comparable to the size of the naturally occuring single crystal grains which usually constitute the polycrystalline films grown on smooth amorphous substrates. The possibility of orienting crystalline overlayers with such structures can be seen if the characteristics of overlayers deposited on amorphous substrates are considered.

Overlayers of crystalline material deposited on smooth amorphous substrates tend to form polycrystalline films $1,2,3$. These overlayers are thus composed of small regions or grains of single crystal separated by grain boundaries. Depending upon the overlayer material, the substrate, and the deposition conditions the size of the single crystal grains can vary from a few tenths of a nanometer to several micrometers (i.e., from nearly amorphous to large grain polycrystalline). Relative to one another the grains tend to be more or less randomly oriented. Under certain deposition conditions and sometimes following annealing of overlayers a single crystallographic direction may become highly preferred ${ }^{1,3}$. Such polycrystalline films which are not entirely random are said to have a fiber texture, (from a term which originated in work with polycrystalline natural fibers that exhibit preferred orientations). The orientations of the preferred direction in these fiber textures is usually associated with the only unique direction on the smooth amorphous substrate, namely, the substrate normal. (In some cases where the mobility of the 
deposit is very low the orientation can be determined by the deposition direction ${ }^{3}$ ). Thus, some particular crystallographic plane of each of the single crystal grains making up the overlayer tends to be parallel to the substrate surface while the crystallographic orientations of the grains in the plane of the substrate surface is random. This phenomenon has been explained using a simple thermodynamic argument ${ }^{3}$. The preferred plane simply corresponds to the plane at which the minimum interfacial tension occurs between the overlayer material and the substrate. For a smooth featureless substrate this interfacial tension is obviously independent of the orientation of the overlayer material in the plane of the substrate, thus there is no preferred "azimuthal" orientation in the substrate plane (i.e., the surface is isotropic in the substrate plane). However, if the substrate surface is not featureless but is instead characterized by some coherent surface relief structure, the surface will have certain unique directions (i.e., it will be anisotropic). For example, assume that this surface-relief structure is composed of a periodic array of planar facets such that the angles between the facets correspond to the angles between the planes of minimum interfacial tension. There can then be unique orientations of the overlayer with respect to the surface relief structure on the substrate which minimize the total interfacial tension. This point is illustrated in Fig. 1-1 for a cubic crystalline overlayer material and an amorphous substrate material where the $\{100\}$ planes of the cubic material parallel to the smooth amorphous surface correspond to the planes of minimum interfacial tension. The square-wave and saw-tooth surface relief structures as illustrated would 

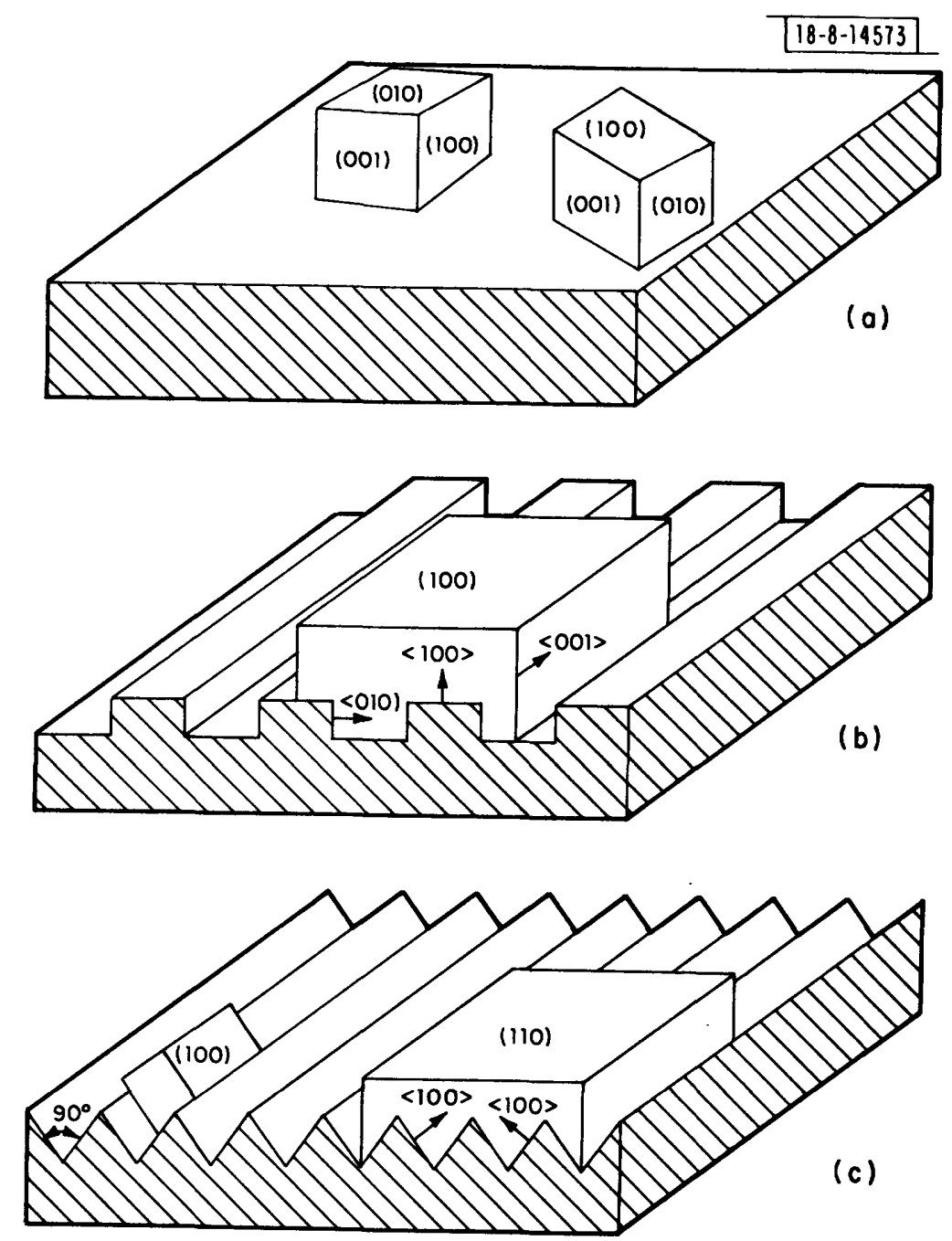

Fin. 1-1. A schematic illustration of the equilibrium orientations of a cubic material on an amorphous substrate with a surface structure, where the interfacial tension is a minimum for the $\{100\}$ family of nlanes parallel to a smooth surface of the amorphous substrate. a). On a smooth substrate, $\{100\}$ parallel to the substrate plane, random orientation in the plane. b). On a square wave arating, $\{100\}$ parallel to substrate plane, <001> parallel to grooves. c). On a sawtooth grating with facets at 90 degrees, $\{110\}$ parallel to substrate plane, <010> barallel to the grooves. 
be defined on the amorphous substrate such that the surface is composed of a periodic array of facets which meet at 90 degree angles. A deposit of the cubic material would see two unique directions at 90 degrees defined by the normals to the facets. The single crystal grains ordinarily comprising a polycrystalline film on an amorphous substrate would now tend to be constrained to 1 ie in a unique orientation with respect to the grating. For the square wave grating of Fig. 1-1 $\{100\}$ planes would be parallel to both the substrate normal and the grating groove direction. For the sawtooth grating the $\{110\}$ plane would lie parallel to the substrate plane. One would expect that such an orientation effect should occur as long as the spacing between the facets of the surface relief structure is smaller than the natural grain size of the film. Since the periodic-surface-relief structures shown in Fig. 1-1 are coherent over a large area and the orientations of the single crystal grains are constrained locally with respect to the grating structures large area single crystal films should result. Obviously geometries other than those shown in Fig. 1-1 are possible for other sets of crystal planes and for other crystal classes.

The plausibility argument given above for orientation of crystalline overlayers by sur.face relief structures on amorphous substrates is further elaborated in Chapter II. The argument is also generalized to include the orientation of the anisotropic mesophases commonly known as liquid crystals. It is shown that under equilibrium conditions that an overlayer which exhibits an anisotropic interfacial tension can be made to assume a uniform "single crystal" orientation relative to an amorphous 
substrate by the influence of a surface relief structure on the substrate. The applicability of this argument for a real substrate and overlayer combination is determined by the ability of the specific system to approach equilibrium. This issue is discussed in Chapter II as it relates to the choice of overlayer and substrate combinations and the determination of the required characteristics of the surface relief structure for an experimental demonstration of the orientation effect.

It is found in general that the limiting factor in an experimental demonstration of the predicted orientation effects for most overlayers is the type and quality of surface relief structure which can be fabricated. As indicated earlier, the spacing of the facets of structures like those shown in Fig. 1-1 should be smaller than the natural grain size of the overlayer, this usually implies spacings less than one micrometer. The facets of the structure should be smooth and flat on a scale much smaller than their spacing and the edges and corners where the facets meet should be as sharp as possible so that a maximum percentage of the overlayer substrate interface will correspond to minimum interfacial tension planes at the desired orientation. Furthermore, important growth and nucleation effects during deposition of crystalline overlayers can be dramatically affected by sharp edges and corners. This important point, discussed in Chapter $V$, indicates that it would be highly desirable if the radii of curvature at the corners between facets were less than one nanometer. For purposes of discussion, the spacing or period of structures on a surface is termed linewidth. The issues of control of facet flatness and corner and edge curvatures are grouped under the term profile control. 
Thus, for this work, linewidths must be smaller than one micrometer and profile control should be such as to provide well defined planar facets oriented at the desired angles with minimum radii of curvature at edges.

Before this research began several techniques using the planar process capable of defining submicrometer linewidths had been developed, including; scanning electron beam lithography, holographic lithography, optical projection and contact photolithography, and soft X-ray 1 ithography ${ }^{4}$. However, few researchers had considered the issue of profile control at the dimensions required here. Research for this thesis revealed that the existing technology could not provide adequate profile control and new techniques had to be developed. Because successful surface relief structure fabrication is a prerequisite for all further experiments this technology development has constituted a major part of the thesis research. Several new techniques were developed during this work which made possible the fabrication of $160 \mathrm{~nm}$ linewidth square-wave grating structures in amorphous $\mathrm{SiO}_{2}$ with smooth faces and radii of curvature at the edges and corners less than $5 \mathrm{~nm}$. The development of these techniques and the details of the new processes are presented in Chapter III. Of great importance in this work is the ability to observe and characterize the structures which have been fabricated. The techniques employed to do this and the results obtained are also given in Chapter III.

The plausibility arguments given for orientation effects in Chapter II indicate that even the simple square-wave-grating surface-relief structures in $\mathrm{SiO}_{2}$ which can be fabricated using the techniques described in Chapter III could be used to induce orientation of many different anisotropic 
overlayer materials. However, a particularly convenient class of overlayer materials for a first test of the concept are liquid crystals. The molecules of these ordered mesophases are highly mobile and thin overlayers can easily approach a near equilibrium configuration ${ }^{5}$. A model of surface relief orientation effects for liquid crystals is given in Chapter IV and an experimental demonstration of orientation of nematic and smectic $A$ liquid crystals by square-wave gratings in $\mathrm{SiO}_{2}$ is described.

In Chapter $V$ a model of the growth of overlayers of solid crystalline materials by thin film deposition on square-wave gratings is presented. Also presented are the results of an experimental investigation of the growth of potassium chloride, tin, and gold on square-wave gratings in $\mathrm{SiO}_{2}$. The model applies to a large number of deposition processes where growth proceeds by nucleation, growth, and coalescence of discrete deposit islands. The model predicts that under certain deposition conditions a solid crystalline overlayer should assume the single crystal equilibrium orientation with respect to a surface relief structure predicted by the arguments given in this introduction and in Chapter II. The orientation effect is demonstrated by the deposition of $\mathrm{KCl}$ from aqueous solution onto a $320 \mathrm{~nm}$ period square-wave grating in $\mathrm{SiO}_{2}$. As predicted the $\mathrm{KCl}$ crystallites grow with their $\{100\}$ planes parallel to the substrate plane and with a $<100>$ direction parallel to the groove direction of the surface relief grating. Depositions by sputtering and vacuum evaporation of gold and tin on square-wave gratings in $\mathrm{SiO}_{2}$ are described in Chapter $V$. The results are explained in light of the model of the growth process for 
these materials.

The Conclusion, Chapter VI, summarizes the work and presents some further speculation about the use of surface-relief structures on substrates for the manipulation of nucleation, growth, and orientation of overlayers. 


\section{OVERLAYER ORIENTATION EFFECTS INDUCED BY SURFACE RELIEF STRUCTURES ON AMORPHOUS SUBSTRATES.}

\subsection{INTRODUCTION}

The objective of this chapter is to give some plausibility arguments for orientation effects of surface relief structures on amorphous substrates, the basic idea being that a uniformly aligned overlayer of an anisotropic material can be produced on an amorphous substrate via the influence of a surface relief structure on the substrate.

Some background information about the interfacial tensions of anisotropic materials is given in section 2.2. In section 2.3 a generalized interfacial tension is defired for nonplanar interfaces and it is shown that the orientation at which the minimum of the generalized interfacial tension occurs between an amorphous substrate and an anisotropic overlayer can be specified by properly chosing the shape of the interface. In section 2.4 the equilibrium orientation of a semi-infinite

overlayer is found to correspond to the orientation of minimum generalized interfacial tension. Thus, it is concluded that under equilibrium conditions the orientation of an overlayer can be chosen by defining the appropriate surface relief structure on the substrate.

Considerations in the choice of overlayer-substrate combinations and substrate-surface-relief structure for an experimental demonstration of the orientation effect are discussed in section 2.5 . 


\subsection{ANISOTROPIC INTERFACIAL TENSIONS}

The effects described in this chapter involve the interaction of an amorphous surface and an overlayer with anisotropic properties. It is assumed that the interface between these two materials has a finite thickness. Outside of this interfacial region the materials can be completely characterized by their bulk properties. The thickness and structure of the interface will depend upon the materials and configuration within that region. A very useful thermodynamical quality which can be used to characterize the interface is the interfacial tension, $\gamma(\vec{n})$ which is defined as the reversible work involved in creating unit area of new surface at constant temperature, volume, and total number of moles.

$$
\gamma(\vec{n})=\lim _{d A \rightarrow 0} \frac{d w}{d A}
$$

where $d w$ is the amount of work required to increase the surface area by $d A$ and $\vec{n}$ is a unit vector which specifies the orientation at the interface.

At constant temperature and volume the work done in creating new surface is equal to the change in Helmholtz free energy, $F$, of the whole system $^{6}$. ( $F=E-T S$, where $E$ is the internal energy, $T$ is the temperature, and $S$ is the entropy.) From this one finds that the interfacial tension is related to the Helmholtz free energy per unit area or the specific surface free energy, $f_{s}$, by

$$
\gamma=f_{s}-\Sigma_{i} \mu_{i} \Gamma_{i}
$$


where $\Gamma_{i}$ is the interfacial excess of the $i^{\text {th }}$ component of the system, and $\mu_{i}$ is the corresponding chemical potential ${ }^{6}$. The relationship between $f_{s}$ and $\gamma$ has only been included for completeness and to emphasize that these quantities are not in general equal for multicomponent systems. For all the discussions which follow the distinction between the interfacial tension and the specific surface free energy is not important. Under equilibrium conditions both $\gamma$ and $f_{s}$ would be minimized. Neither $\gamma$ or $f_{s}$ is known completely for any of the material combinations considered in this research. However, the general form of both quantities can be deduced from symmetry, crystal structure and crystal habit ${ }^{6,7,8}$. Of the two quantities the interfacial tension can be directly measured ${ }^{6}$. Thus, in all of the following discussions the interfacial tension will be used. The form of the function $\gamma(\vec{n})$ is of course determined by the details of the interface. For instance, the interfacial tension between liquid-liquid, amorphous solid-liquid, amorphous solid-vapor, and liquidvapor is simply a constant with the units of energy per area, i.e., the surface tension is completely isotropic. On the other hand, interfacial tensions which involve an ordered phase, (where the term ordered phase includes anisotropic mesophases such as liquid crystals as well as solid crystals) are in general functions of the orientation of the ordered phase with respect to the interface. As an example, the interfacial tension, $\gamma(\vec{n})$, between an ordered phase and a non-ordered phase such as a vapor, liquid, or amorphous solid would be a function of the interface orientation specified by the unit vector $\vec{n}$. The variation of $\gamma(\vec{n})$ can, 
in these cases, be conveniently displayed on a polar plot, called a Wulff plot ${ }^{6}$, where the direction of the radius vector, $\vec{r}$, specifies the crystalline orientation of the surface, and the magnitude of the vector is equal to the magnitude of the interfacial tension. i.e.

$$
\vec{r}=\gamma(\vec{n}) \vec{n}
$$

Possible forms of the $\gamma$ plot are listed in the first two columns of table 2-1 and examples are shown in Fig. 2-1.

TABLE 2-1 Possible Forms of the $\gamma$-Plot of the Interfacial Tension Between a Given Material and Non-ordered Phase. (The equilibrium shape of a particle is discussed in Chapter $V_{\text {. }}$ )

\begin{tabular}{|l|l|l|}
\hline Material & \multicolumn{1}{|c|}{$\begin{array}{l}\text { Y Plot } \\
\text { See Figure 2-1 }\end{array}$} & $\begin{array}{l}\text { Equilibrium Shape } \\
\text { See Figure 5-4 }\end{array}$ \\
\hline Normal Liquid... & Sphere & Sphere \\
Liquid Crystal... & Smooth but anisotropic & a) b) \\
Solid Crystal & Finite number of cusps & c) d) e) \\
\hline
\end{tabular}

An important point to notice here is that for the interfaces specified in Table 2-1, which are between an ordered phase and a nonordered phase, the interfacial tension depends only upon the orientation of the ordered phase. If the interface is between two ordered phases the interfacial tension will depend upon the orientation of both of the phases 

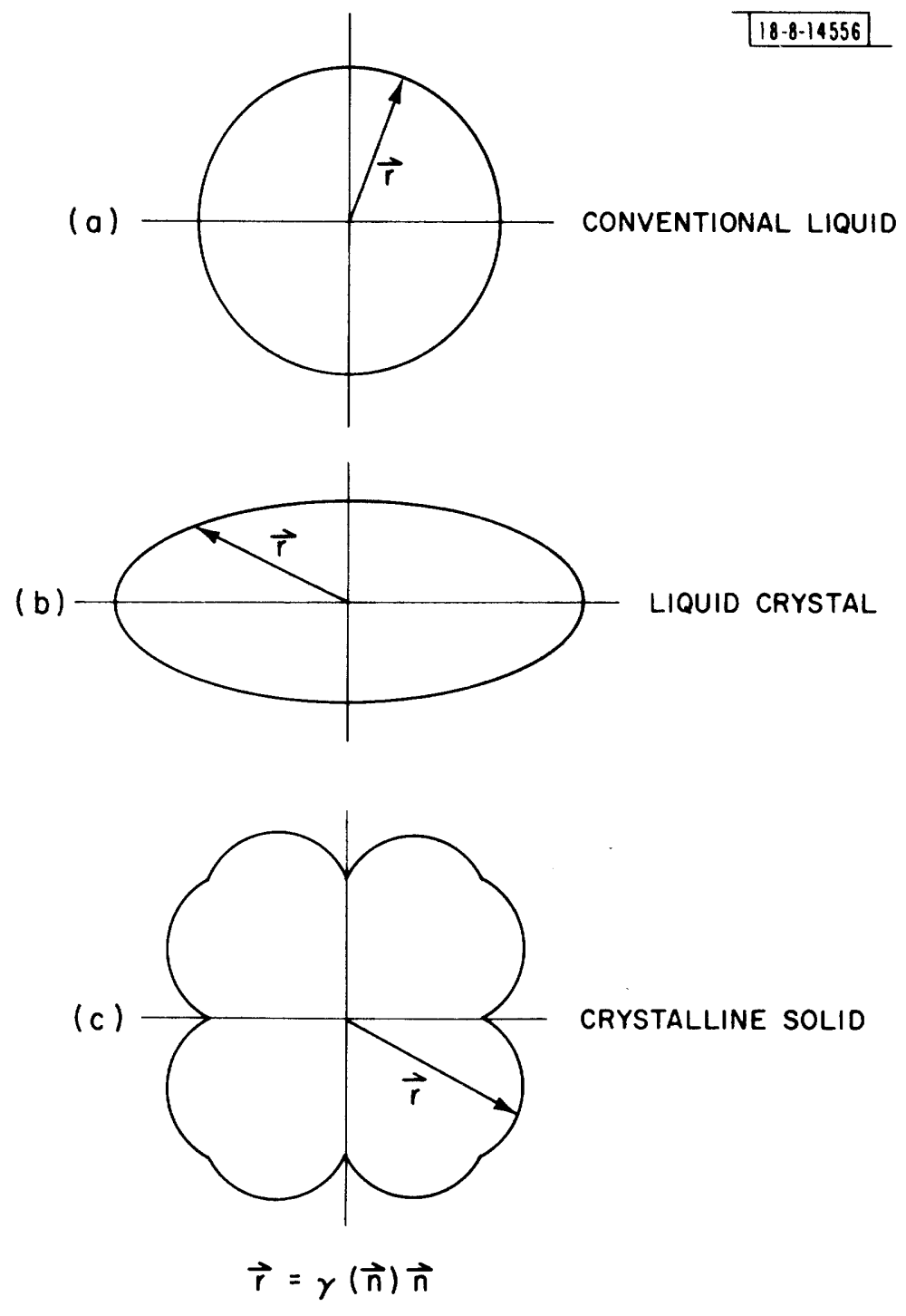

Fig. 2-1. Cross-sections of representative interfacial tension plots are shown for a conventional isotropic liquid, a liquid crystal, and a cubic crystalline solid where the cross-section is a $\{100\}$ plane. 
relative to one another. Obviously, the interfacial tension can not be represented in general on a polar plot in three dimensions when both phases are ordered. In this case the interfacial tension is most conveniently represented by the function $\gamma(\vec{n})$ where $\vec{n}$ uniquely specifies the relative orientation of the phases. Polar plots of various sections of the function $\gamma(\vec{n})$ can obviously be made and are very useful. 


\subsection{GENERALIZED INTERFACIAL TENSIONS FOR SURFACE RELIEF STRUCTURES}

All of the interfaces discussed so far have been assumed to be planar. However, the concept of interfacial tension can be generalized to include nonplanar surfaces as well. As an example, assume that the interface is specified by some periodic function I $(x, y)$. The average surface or substrate plane will simply be the $x-y$ plane. The generalized interfacial tension, $\sigma(\vec{n})$, is then defined as the energy required to create a unit area of new interface in the $x-y$ plane.

$$
\begin{aligned}
& \sigma(\vec{n})=\int \gamma(I(x, y)) d x d y \\
& \text { unit area } x-y \text { plane }
\end{aligned}
$$

where $\gamma(I(x, y))$ is the interfacial tension at the interface $z=I(x, y)$ for the crystalline overlayer orientation specified by $\vec{n}$. (In order that the concept of a generalized interfacial tension be usefur, the unit area must encompass an integral number of "unit cells" of the interface.)

If the periodic boundary $z=I(x, y)$ separates an amorphous substrate and a crystalline overlayer, the interfacial tension will now depend on both the orientation of the overlayer with respect to the interfacial $x-y$ plane and a plane perpendicular to this plane, say the $y-z$ plane. This orientation information can be specified uniquely by three coordinates. However, for purposes of description, a more convenient notation uses two sets of Miller indicies, the first specifying the crystalline direction parallel to the $z$ axis, and the second specifying the direction parallel to the $y$ axis. For instance, $\sigma\left(\langle 100\rangle_{z}\langle 110\rangle_{y}\right)$, specifies the generalized interfacial tension of a surface with a $\{100\}$ plane parallel to the surface 
where the $\langle 110\rangle$ direction is parallel to the $y$ direction in the plane of the interface.

By the introduction of a periodic nonplanar boundary (i.e., a surface relief structure on the substrate), the interfacial tension of an amorphous substrate and a crystalline overlayer has been made anisotropic with respect to orientation of the overlayer in the plane of the substrate. More explicitly, the non-ordered isotropic surface of a planar amorphous substrate has been made "ordered" and "anisotropic" by periodically modulating the surface.

To illustrate the form of an interfacial tension of an anisotropic surface and also to show how a surface-relief structure on an amorphous substrate can introduce an anisotropy such that a well defined minimum in $\sigma(\vec{n})$ occurs at a desired orientation, the hypothetical interfaces illustrated in Fig. 2-2 are considered.

The interface between an amorphous material ( $\mathrm{SiO}_{2}$ for instance) and a cubic material (such as an alkali halide) is described by a square-wave function or sawtooth function of period, $s$, and height, $h$, in the $x$ direction. Assume for simplicity that there is no variation in the $y$ direction. The interfacial tension for the interfaces of Fig. 2-2 can be constructed as a weighted sum of the interfacial tensions for plane interfaces, assuming the line tensions associated with the edges and corners can be ignored. A polar plot is shown in Fig. 2-3 of the generalized interfacial tension, $\sigma_{q}(\vec{n})$, calculated in this way, for the square-wave structure of Fig. 2-2(a) where the interfacial tension of a plane interface of these materials varies with orientation in the manner 


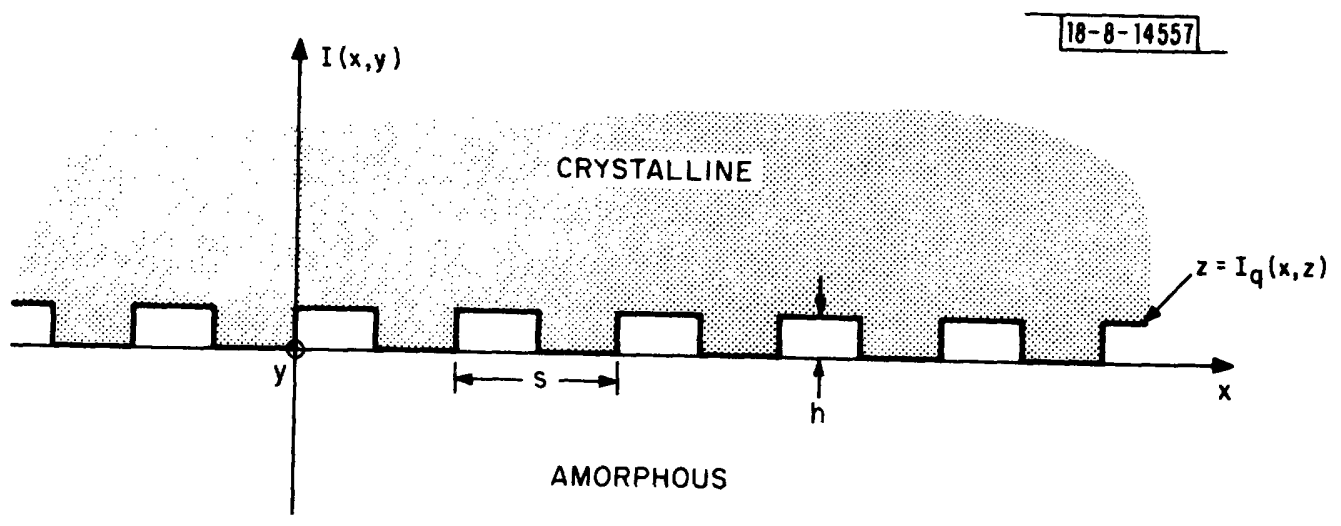

(a)

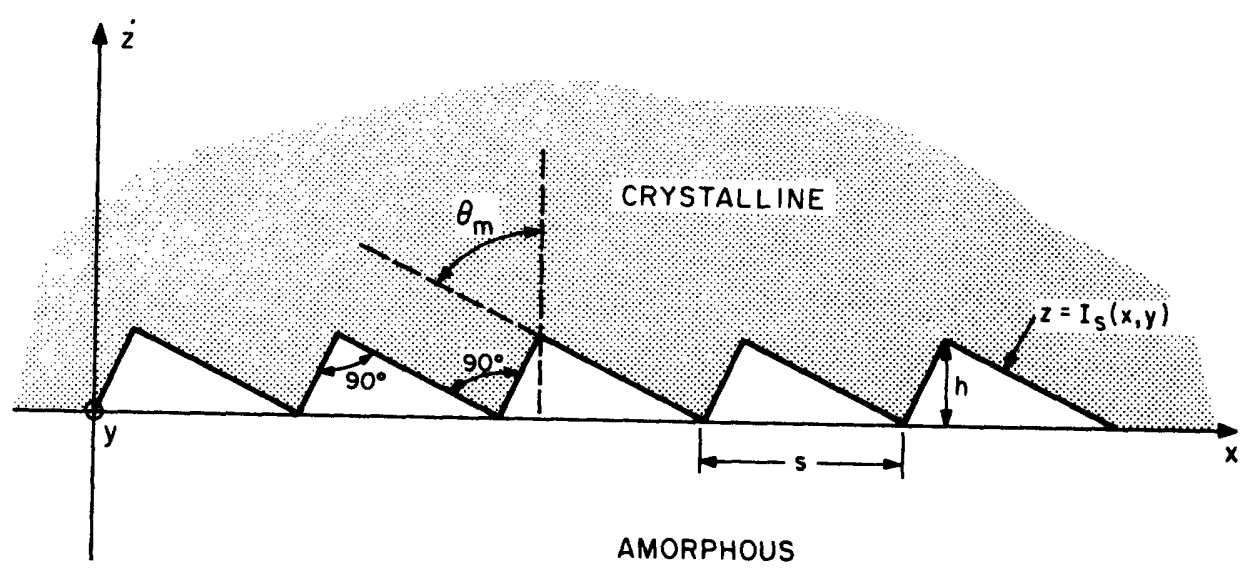

(b)

Fig. 2-2. Schematic cross-sections are shown of a square-wave interface and a sawtooth interface between an amorphous and a crystalline material. There is no variation in the $y$ direction. 

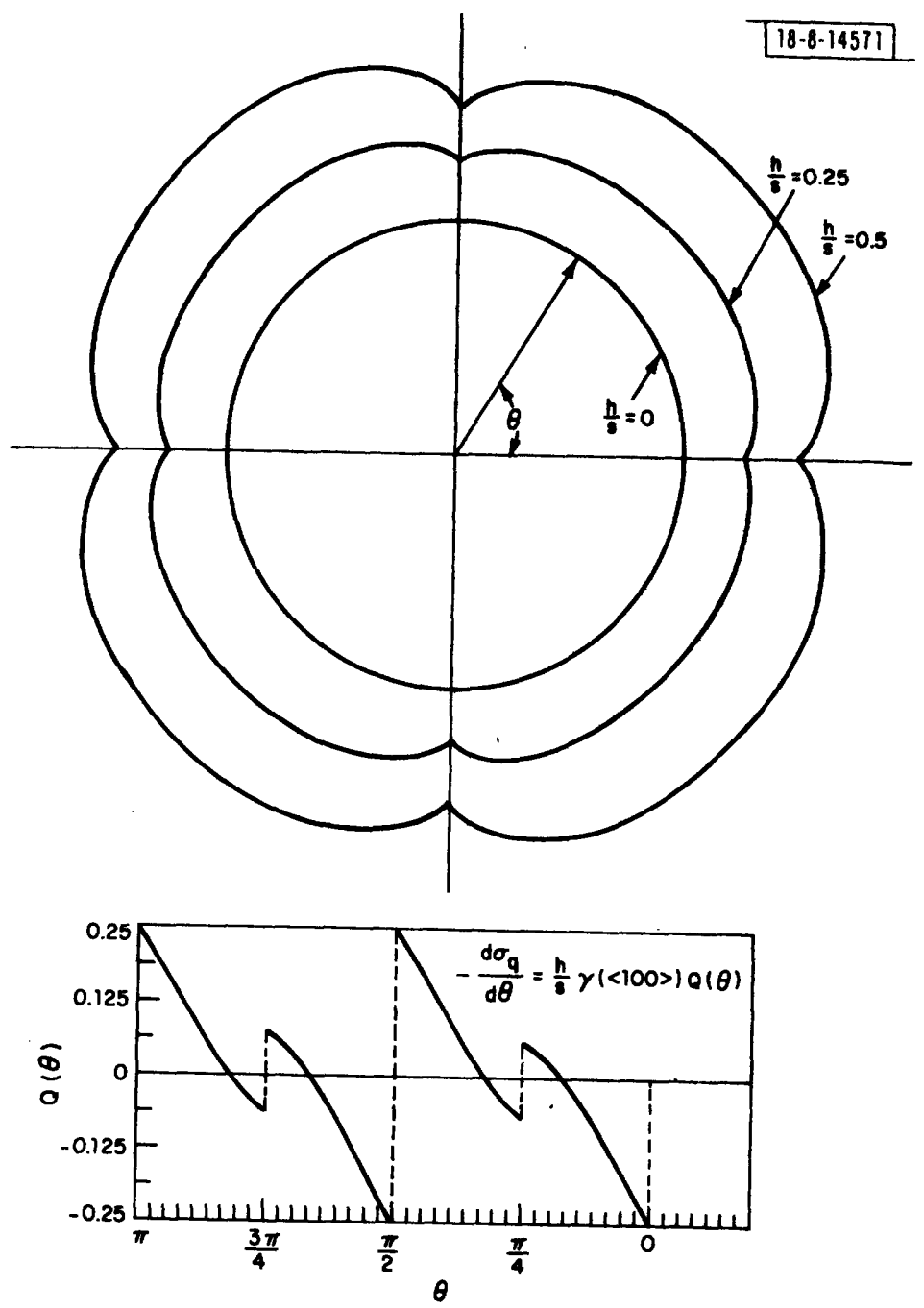

Fig. 2-3. A folar plot is shown of the generalized interfacial tension, $\sigma_{q}$, of the square wave interface depicted in Fig. 2-2(a) where $\gamma(\vec{n})$ of the crystalline material is given in Fig. 2-1(c). The portion of the function $\sigma_{q}(\vec{n})$ shown is for $\{100\}$ parallel to the substrate where $\theta$ measures the deviation of $\langle 010\rangle$ from the $y$ direction. Also shown is a plot versus $\theta$ of the "orientation force" $-\frac{d \sigma_{q}}{d \theta}$. 
illustrated by the plot in Fig. 2-1(c). The plot is shown for a $\{100\}$ plane of the cubic material fixed parallel to the $x-y$ plane as a function of $\theta$, the angle of deviation of the $\langle 010\rangle$ direction from the $y$ direction. Several plots are shown, each corresponding to a different value of the parameter $\mathrm{h} / \mathrm{s}$. Note that there will be four identical minima which occur at $\theta$ equals $0, \frac{\pi}{2}, \pi, \frac{3}{2} \pi$ and $\{100\}$ parallel to the $x-y$ plane. (i.e., $\left.\sigma_{q}\left(\langle 100\rangle_{z}<010\right\rangle_{y}\right)$ is minimum. $)$

Also plotted in Fig. 2-3 is the in-plane "orientation force", $-\frac{d \sigma q}{d \theta}$.

Obviously the magnitude of $\frac{\mathrm{d} \sigma_{\mathrm{q}}}{\mathrm{d} \theta}$ increases as the parameter $\mathrm{h} / \mathrm{s}$ increases.

Given that $\gamma(\vec{n})$ of the crystalline material corresponds to Fig. $2-1(c)$ where $\gamma(\langle 100\rangle)$ is minimum, it is clear that the minimum of the generalized interfacial tension, $\sigma_{s}$, for the sawtooth structure of Fig. 2-2(b) will occur for a $<100>$ direction parallel to the $y$ or groove direction and another $\langle 100\rangle$ direction at an angle $\theta_{\mathrm{m}}$ with respect to the substrate normal. Thus $\theta_{m}$ can be chosen such that $\sigma_{s}\left(\langle h k 0\rangle_{z}\langle 100\rangle_{y}\right)$ is minimum for any $h$ and $k$. By modulating the surface in the $y$ direction in a similar sawtooth manner the minimum of $\sigma_{s}$ can be chosen to occur at any crystal plane parallel to the substrate.

It is evident that for a given $\gamma(\vec{n})$ between a crystalline material and an amorphous substrate a periodic surface relief structure could be found such that the minimum of $\sigma(\vec{n})$ occured at a unique orientation in the substrate plane. 


\subsection{ORIENTATION EFFECTS AT EQUILIBRIUM}

Given the interfacial tensions $\gamma(\vec{n})$ for a specific system the equilibrium configuration can be found. At a fixed temperature and total volume the condition of equilibrium is one of minimum Helmholtz free energy ${ }^{6}$, or $\quad \int \begin{aligned} & \gamma(\vec{n}) d A \text { is minimum } \\ & \text { Interface }\end{aligned}$

Where $\gamma(\vec{n})$ is the ordinary interfacial tension of an interface orientation specified by the vector $\vec{n}$ and the integral is taken over the entire interfacial region.

To illustrate the application of equation (2.5) consider a system made up of a semi-infinite amorphous substrate, an overlayer of anisotropic material of finite thickness and a semi-infinite "superstrate" region above the overlayer. The integral of equation (2.5) would be taken over the upper and lower overlayer interfaces. If the substrate and superstrate interfaces are identical, equation (2.5) lends simply to the requirement at equilibrium that

$$
\sigma(\vec{n}) \text { is minimum }
$$

If the interfaces are different the requirement becomes

$$
\left(\sigma(\vec{n})_{\text {upper }}+\sigma(\vec{n})\right)_{\text {lower }} \text { is minimum }
$$

For most solid overlayers deposited on substrates the upper surface of the overlayer would be unconfined and would be in contact with the non-ordered parent phase of the deposit. If the minimum interfacial tension of the 
overlayer and its parent phase occur for the same plane parallel to the superstrate as the substrate the equilibrium requirement reduces once again to equation (2.6). This will be the case for all solid crystalline overlayers considered in this thesis. (In general it would be necessary to minimize equation 2.5 for all variations of overlayer orientation.)

The consequences of equation (2.5) can be seen by considering some specific material combination. For the case $\gamma$ is isotropic (such as in amorphous solid-liquid etc.) all orientations are of course equivalent. In the instance where the interfacial tension, $\gamma(\vec{n})$, is a function solely of the orientation of the overlayer material with respect to the substrate normal, all directions in the plane of the interface are equivalent. An example is the case of a crystalline material deposited on a smooth amorphous substrate where equation (2.5) requires that the minimum interfacial tension plane be parallel to the amorphous surface, but there is no preferred orientation in the plane. In real systems one could expect that locally the overlayer will assume the equilibrium single crystal orientation with the minimum interfacial tension plane parallel to the substrate while over longer distances on the substrate, the in-plane orientation of the overlayer will tend to wander. This leads to the fiber textures observed in crystalline deposits on amorphous substrates discussed in the introduction and Chapter V.

If the interfacial tension has a minimum at some unique orientations of overlayer and substrate then the equilibrium orientation will be unique. (In addition to the true minima of $\sigma(\vec{n})$ there may also be relative minima such that the system can exist in a metastable equilibrium.) In nature 
it appears that there exist some interfaces between two crystals which have strong and unique minima in their interfacial tensions. This may manifest itself in the phenomenon of heteroepitaxy where oriented single crystal overgrowth of a material occurs on a specific crystal plane of another material ${ }^{1,2}$. As an example, a system which has been widely studied is gold on rocksalt $(\mathrm{NaCl})$ where a $\{100\}$ plane of the gold grows parallel to a $\{100\}$ plane of the rocksalt and a $\langle 100\rangle$ direction of gold is parallel to a $<100>$ direction of $\mathrm{NaCl}$ in the plane of the interface. Of course heteroepitaxy does not occur smooth amorphous substrates, but as shown in section 2.3 an interface between an amorphous substrate and a crystalline overlayer can be made anisotropic which characteristics analogous to a single crystal surface by the introduction of a periodic surface relief structure on the substrate such that the generalized interfacial tension can have a unique minimum. Equations (2.6) and (2.7) indicate that an overlayer on such a substrate should assume the orientation corresponding to the minimum of generalized interfacial tension. Since this overlayer-substrate orientation would be uniform and unique, in effect, heteroepitaxy would be obtained on an amorphous substrate. The idea advanced in this thesis is that under suitable conditions real systems can approach this idealized equilibrium configuration and oriented single-crystal overgrowths can be induced on amorphous substrates by surface relief structures. 


\subsection{CONSIDERATIONS IN THE CHOICE OF OVERLAYER-SUBSTRATE COMBINATIONS AND SUBSTRATE SURFACE RELIEF STRUCTURE FOR AN EXPERIMENTAL DEMONSTRATION OF THE ORIENTATION EFFECT}

In the previous sections of this chapter it was shown that at equilibrium it should be possible to impart a desired orientation to an anisotropic overlayer on an amorphous substrate by defining an appropriate surface relief structure on the substrate. To assess the possibility of experimentally demonstrating the predicted effect, the elements required for an experimental investigation must be considered.

i) An overlayer material which exhibits an anisotropic interfacial tension, preferably a material with large anisotropy.

ii) A well-controlled and characterized surface-relief structure on an amorphous substrate with the required dimensions and shape.

iii) A convenient deposition technique for applying the overlayer material to the substrate such that the overlayer can approach the equilibrium configuration during deposition. (Another possibility is that the overlayer could approach equilibrium after deposition by being "annealed").

iv) A convenient unambiguous evaluation technique capable of determining the orientation of the overlayer with respect to the substrate surface relief structure.

Although a great many solid crystalline and liquid crystal materials exhibit anisotropic interfacial tensions, only a restricted class of materials are compatible with the shape of the surface relief structures which can be fabricated at the present time. As will be shown in Chapter III fabrication 
techniques have been developed which permit the fabrication of wellcontrolled square-wave-grating surface-relief structures in amorphous $\mathrm{SiO}_{2}$. (The structures illustrated in Figs. 1-1(b) and 2-2(b) could represent idealizations of the real structures which can be fabricated.) For a well defined minimum to occur in the generalized interfacial tension of the overlayer material and the square-wave structure such that the equilibrium orientation of the overlayer would be unique, the planes of minimum interfacial tension for the overlayer material must naturally occur at right angles to one another. As an example, if the $\{100\}$ or $\{110\}$ planes of a cubic overlayer material were the planes of minimum interfacial tension, the generalized interfacial tension would have a minimum at $\{100\}$ or $\{110\}$ parallel to the substrate plane and $\langle 100\rangle$ parallel to the grating groove direction. Another possibility would be a tetragonal overlayer material where $\{100\}$ is the plane of minimum interfacial tension. In this case the minimum of the generalized interfacial tension would occur for $\{100\}$ parallel to the substrate plane and $<001>$ parallel to the grating groove direction. For some liquid crystals, any plane parallel to the liquid crystal director or c-axis of the liquid crystal can correspond to a plane of minimum interfacial tension ${ }^{5}$. The minimum of generalized interfacial tension for those liquid.crystals on a square-wave grating structure would occur for the c-axis parallel to the plane of the substrate and parallel to the grating groove direction.

With the required material properties in mind the following overlayer materials were chosen for this first investigations of the orientation 
effect.

i) Liquid crystals - MBBA and M-24

ii) Alkali halides - KCl

iii) Metals - Tin

The argument presented in section 2.4 for the orientation effect was an equilibrium argument and its applicability to practical situations depends upon the ability of a real deposit-substrate system to approach equilibrium. Liquid crystals are known to exhibit highly anisotropic interfacial tensions. The liquid crystal molecules are also highly mobile and an equilibrium configuration in a thin overlayer is more easily achieved than for solid crystal systems. For these reasons liquid crystals are well suited for the initial overlayer orientation experiments. The alkali halides were determined to be an excellent choice for the first orientation experiments with solid crystalline materials because they have a cubic structure with a minimum of $\gamma(\vec{n})$ at $\{100\}$ parallel to $\mathrm{SiO}_{2}{ }^{3}$. Also the alkali halides are known to have much more anisotropic interfacial tensions than other cubic materials such as metals ${ }^{1,6}$. The alkali halides are easily depasited from an aqueous solution by a very simple evaporation technique or by electron beam evaporation in a high vacuum. Because $\mathrm{KCl}$ is water soluble and is easily removed, depositions can be performed several times on the same substrate.

Vacuum evaporated Tin, which has a tetragonal structure, exhibits a $\{100\}$ fiber texture when deposited on smooth amorphous $\mathrm{SiO}_{2}$ substrates ${ }^{3}$. This indicates that the $\{100\}$ planes are the planes of minimum interfacial tension. Tin has a low melting point which lends to a relatively high 
atomic mobility, it also can be annealed ${ }^{7,8}$ so as to approach equilibrium at modest temperatures of $100-200{ }^{\circ} \mathrm{C}$.

Although the underlying principle of overlayer orientation by surface relief structures is the same for both liquid crystals and solid crystalline materials the detailed models of the orientation effect for each material are quite different. The modeling of surface-relief-structure-induced orientation effects for liquid crystals and liquid crystal orientation experiments are discussed in Chapter IV. A detailed model of orientation of solid crystalline overlayers is presented in Chapter $V$. Because the atoms in a solid deposit are much less mobile than those of a liquid crystal, very careful consideration of the mechanism of deposition is necessary in the case of solid overlayers. In Chapter $V$ both deposition from aqueous solution and by vacuum evaporation are treated by the same model of thin film nucleation and growth.

A prerequisite for all orientation experiments is the availability of a well-controlled and characterized surface-relief structure on an amorphous substrate. The idealized square-wave structure shown in Figures 1-1(b) and 2-2(a) was assumed to have perfectly smooth facets oriented at exactly $90^{\circ}$ to one another joined by infinitely sharp edges. The facets of a real structure will have some finite roughness and they will not be oriented at exactly $90^{\circ}$ to one another. The edges will have some finite curvature. As shown in Fig. 2-3 for a given height, $h$, the anisotropy and orientation force of the square wave structure decreases as period s increases. Thus, in a real system, the structure will have some maximum 
period at which it will be effective in inducing overlayer orientation. The detailed models of thin solid film depositions indicate that squarewave grating periods less than one micrometer (corresponding to the natural grain size of polycrystalline overlayers of these materials) would be needed to induce single crystal overlayer growth. As the roughness of the facets increase and the facets deviate from intersection angles of $90^{\circ}$, and the sharpness of the edges decreases the anisotropy of the generalized interfacial tension of the overlayer and the surface relief structure will decrease. A priori it is difficult to place a limit on the maximum acceptable deviation from the idealized square wave structure. In order that the square-wave structure remain well defined, the roughness of the facets should probably deviate less than $10 \%$ of the structure period, and the sidewall angles should deviate less than $10 \%$ from $90^{\circ}$. Obviously the radius of curvature of the grating edges must be less than the height of the grating if it is to approximate a square wave. The thin solid film deposition model of Chapter $V$ indicates that important nucleation phenomenon could be induced by very sharp grating edges. For this reason radii of curvature as small as $0.1 \mathrm{~nm}$ would be desirable. In the case of liquid crystals a model indicates that the anisotropy of the generalized interfacial tension increases rapidly as the edge curvature decreases. The problem of fabricating and characterizing square-wave structures on amorphous substrates with the required dimensions is addressed in Chapter III. 


\section{FABRICATION OF SURFACE RELIEF STRUCTURES}

\subsection{INTRODUCTION}

The fabrication method used in this research has become known as the planar process. This very flexible technique has a long history and has been used for many years in the fields of art and printing. Recently the use of the planar process in the manufacture of integrated circuits has lead to a great deal of research into the capabilities of the process itself. As a result of this work high resolution techniques have been developed which make possible the fabrication of submicrometer structures. An excellent review of these advances has been published by $\mathrm{Smith}^{4}$. Despite the recent achievements in fabrication technology, prior to this thesis research, the ability to fabricate structures with the properties described in the introduction and section 2.5 had not been demonstrated. Because existing fabrication techniques were found to be inadequate for this work new methods were developed. The theory and practice of these new techniques as applied to the fabrication of grating type surface relief structures on amorphous silicon dioxide $\left(\mathrm{SiO}_{2}\right)$ for use in crystalline overlayer orientation experiments are described in this Chapter.

The basic fabrication steps used in the planar process are as follows. The substrate on which the structure is to be fabricated is coated with a layer of radiation sensitive polymer (resist). The resist is then exposed to a pattern of radiation. (Exposure to radiation either increases or decreases the solubility of the resist in its developer for 
positive and negative resists respectively.) After exposure, the resist is immersed in developer, the resulting resist pattern can then act directly as a mask during substrate etching or serve to define a pattern by the liftoff technique 4 . Many combinations of etching, liftoff, and multiple pattern exposure are of course possible. The specific fabrication process developed during the thesis work to produce the basic grating structure used for most overlayer orientation studies is shown schematically in Fig. 3-1.

The process is comprised of five major steps:

1. Mask pattern generation by holographic exposure and ion-beam etching.

2. Pattern replication by X-ray lithography.

3. Liftoff of chrome.

4. Reactive-ion-etching.

5. Final cleaning.

In the next sections each of these steps is discussed in detail. 


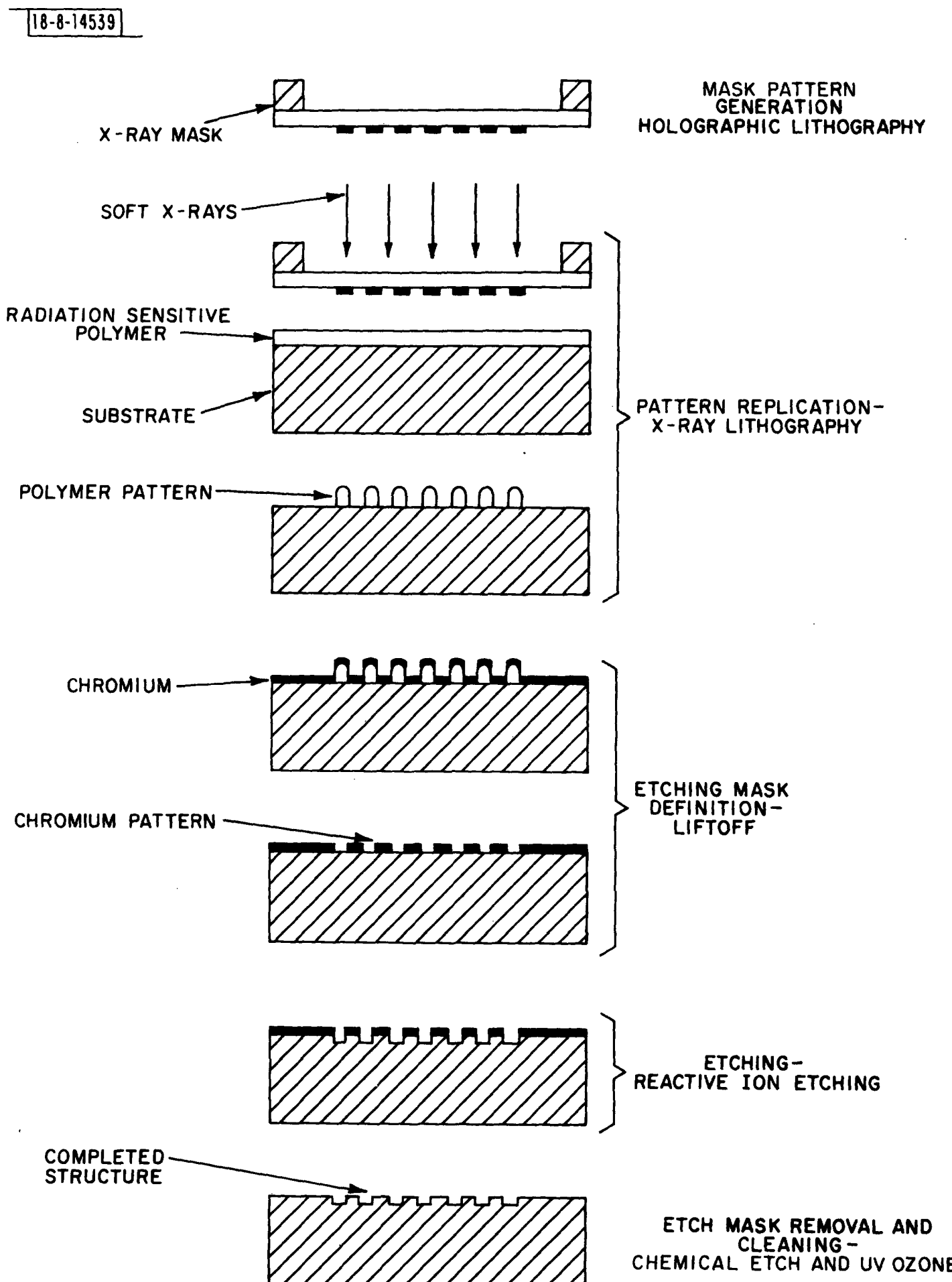

Fig. 3-1. A schematic illustration of the process developed for fabricating submicrometer period square-wave grating surface-relief structures in amorphous $\mathrm{SiO}_{2}$. 


\subsection{MASK PATTERN GENERATION BY HOLOGRAPHIC LITHOGRAPHY}

To generate a grating-type surface relief structure by the planar process depicted in Fig. 3-1 an X-ray mask pattern must first be generated. At the present time, only two pattern generation techniques are capable of directly producing polymer relief gratings with spatial periods smaller than $500 \mathrm{~nm}$, these are scanning electron beam lithography (SEBL) and holographic lithography. The use of an existing SEBL system at Lincoln Laboratory was considered for this work and rejected because of electron backscattering problems ${ }^{9}$ and the extremely long exposure times needed to produce gratings of $100 \mathrm{~nm}$ linewidth over the desired $1 \mathrm{~cm}^{2}$ areas.

Holographic 1 ithography, first proposed by Rudolph and Schmahl ${ }^{10}$, is well suited for producing large area grating patterns. The apparatus required is simple and the exposure times are short $(10-60 \mathrm{sec})$. However, during this research the technique was found to have several drawbacks which limit its use as a final lithography step in the fabrication of square-wave grating structures in $\mathrm{SiO}_{2}$. These drawbacks include, limited resist profile control as a result of substrate reflection, intrinsic granularity of the photoresist, and poor adhesion of photoresist to $\mathrm{SiO}_{2}$ surfaces. Nevertheless holographic lithography can be used for pattern generation during fabrication of the gold absorber patterns used in the $X$-ray masks described in section 3.3.4.

The apparatus used for holographic lithography exposures is shown schematically in Fig. 3-2. The beam from an argon ion laser (457.9 nm) is passed through a spatial filter and beam expander, and split into two 


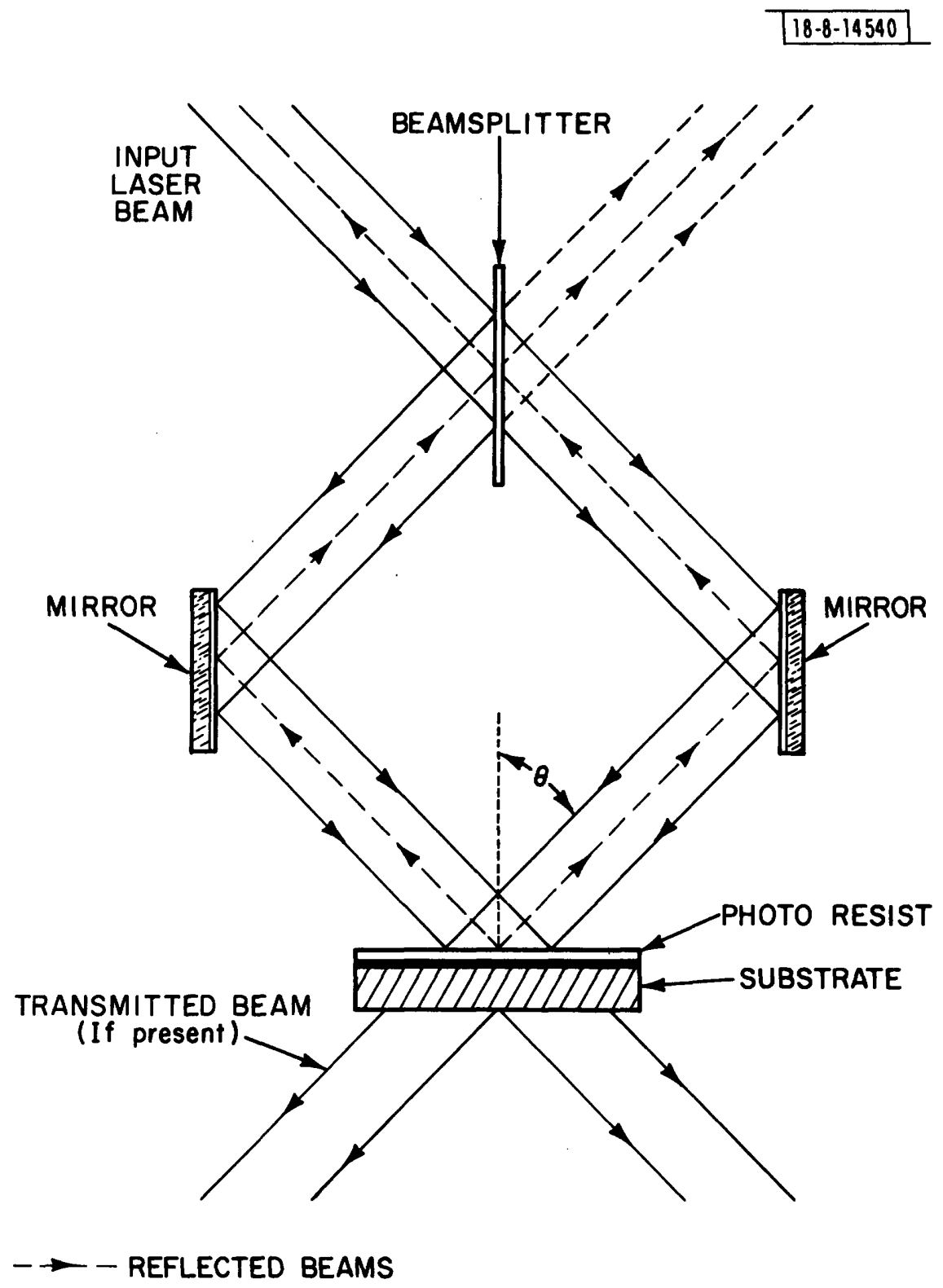

Fig. 3-2. A schematic illustration is shown of the configuration of optical compoenents used to expose gratings in Shipley AZ-1350 photoresist by interfering two laser beams. 
plane wave beams of equal intensity. These beams are then reflected from a pair of mirrors arranged so as to bring the two beams together at a known angle on a photoresist coated substrate. The two laser beams interfere to produce a sinusoidal intensity pattern in the plane of the substrate with a spacing given by

$$
s=\frac{\lambda}{2 \sin \theta}
$$

Where $s$ is the grating period, $\lambda$ is the wavelength of the radiation and $\theta$ is the angle between the beams and the substrate normal.

In the case of a reflective substrate surface the reflected waves will interfere with the incident waves and the intensity pattern will also vary sinusoidally in the direction normal to the substrate surface. The intensity will be approximately zero at the interface between the resist and a highly reflective substrate leading to under exposure at the interface. In general, to insure optimum exposure on a reflective substrate the thickness of the photoresist, $t_{r}$, should be less than one half the spatial period of the intensity pattern in the direction normal to the substrate or where $n_{r}$ is the index of refraction of the photoresist,

$$
t_{r}<\frac{\lambda}{4 n_{r} \sqrt{1-\left(-\frac{\lambda}{2 s n_{\eta}}\right)^{2}}}
$$

The smoothness of the final pattern is of great importance as indicated in section 2.5. The intensity pattern of two interfering laser beams is expected to be extremely smooth, thus the intrinsic smoothness of the resist will determine the amount of edge ripple in the exposed 
resist pattern. An SEM micrograph is shown in Fig. 3-3 of the type of photoresist pattern obtained on a reflective substrate. The photoresist is Shipley $A Z-1350 \mathrm{~J}^{11}$, a high resolution positive photoresist, which has been studied extensively by Dill and others ${ }^{12}$. Note that the intrinsic roughness of the resist appears to be $\approx 10 \mathrm{~nm}$. The resist thickness of $100 \mathrm{~nm}$ was obtained by diluting AZ-1350J resist with AZ thinner and spin-coating at $5000 \mathrm{rpm}$ for $30 \mathrm{sec}$. The substrate material is a thin film of gold. The incident exposure energy was $\approx 0.1$ joule $/ \mathrm{cm}^{2}$. The development was by immersion for $15 \mathrm{sec}$. at $20{ }^{\circ} \mathrm{C}$ in a $1: 1$ mixture of $A Z$ developer: $\mathrm{H}_{2} \mathrm{O}$.

The rounded photoresist profile shown in Fig. $3-3$ is useful as an ion-beam etching mask for materials such as gold which etch much faster than the photoresist, but such profiles are not adequate to define the desired square profiles in low etch-rate materials such as $\mathrm{SiO}_{2}$. Attempts to produce a square profile in Shipley AZ-1350 resist by holographic lithography were frustrated by back reflection from the substrate, which causes an unavoidable modulation in the intensity pattern of the interfering laser beams in the direction normal to the substrate surface.

It was found that by using a simultaneous exposure and development technique ${ }^{13,14}$ (which enhances resist nonlinearity) and a transparent substrate with nearly the same refractive index as the photoresist, a nearly square profile can be obtained. A SEM micrograph of this result is shown in Fig. 3-4. Unfortunately this technique could not be used as a final lithography step in the fabrication process outlined in Fig. 3-1. 


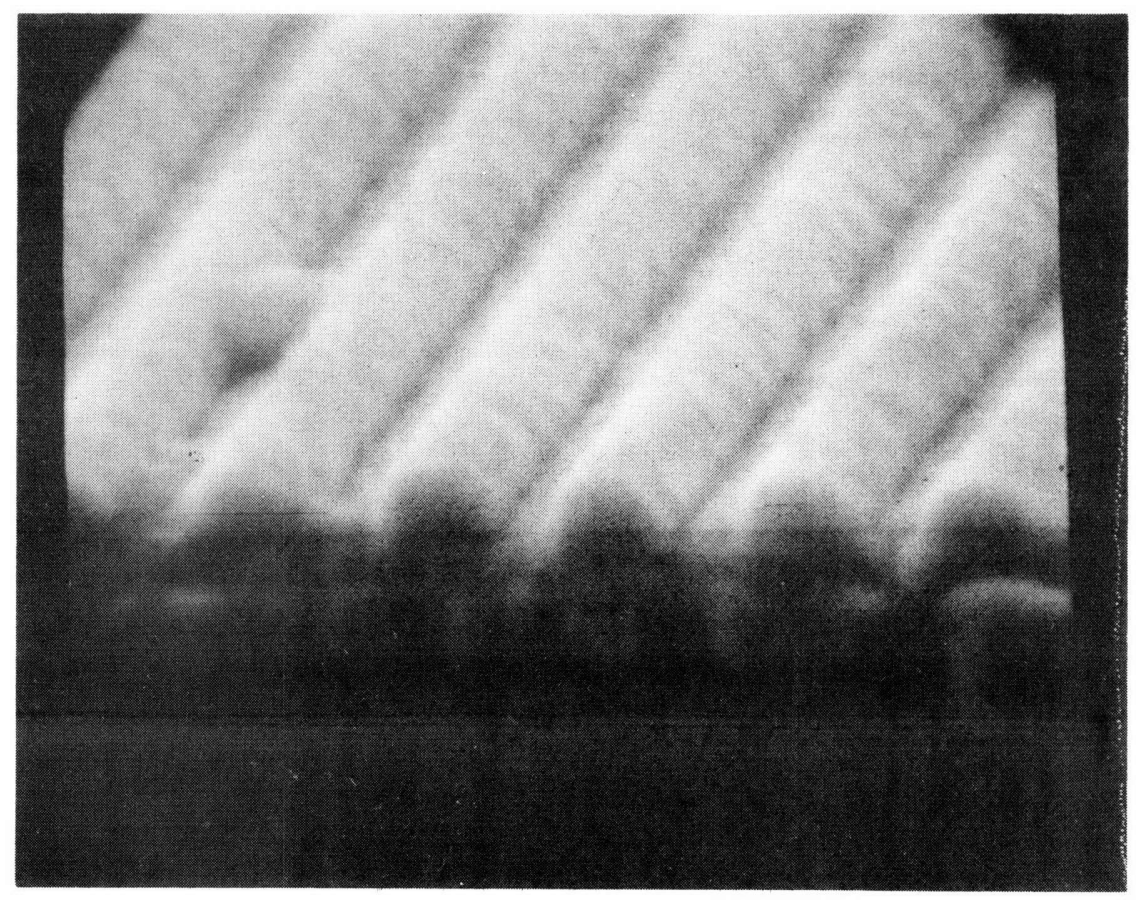

Fig. 3-3. An SEM micrograph of a holographic exposure of $A Z-1350$ photo resist on a $100 \mathrm{~nm}$ thick gold film. The grating period is $320 \mathrm{~nm}$, and the resist thickness $100 \mathrm{~nm}$. The exposing wavelength was $457.9 \mathrm{~nm}$ and the total exposure energy density was $\approx 0.1$ joule $/ \mathrm{cm}^{2}$. Development was by immersion for $15 \mathrm{sec}$. in a 1 to 1 solution of $A Z$-developer and water. 


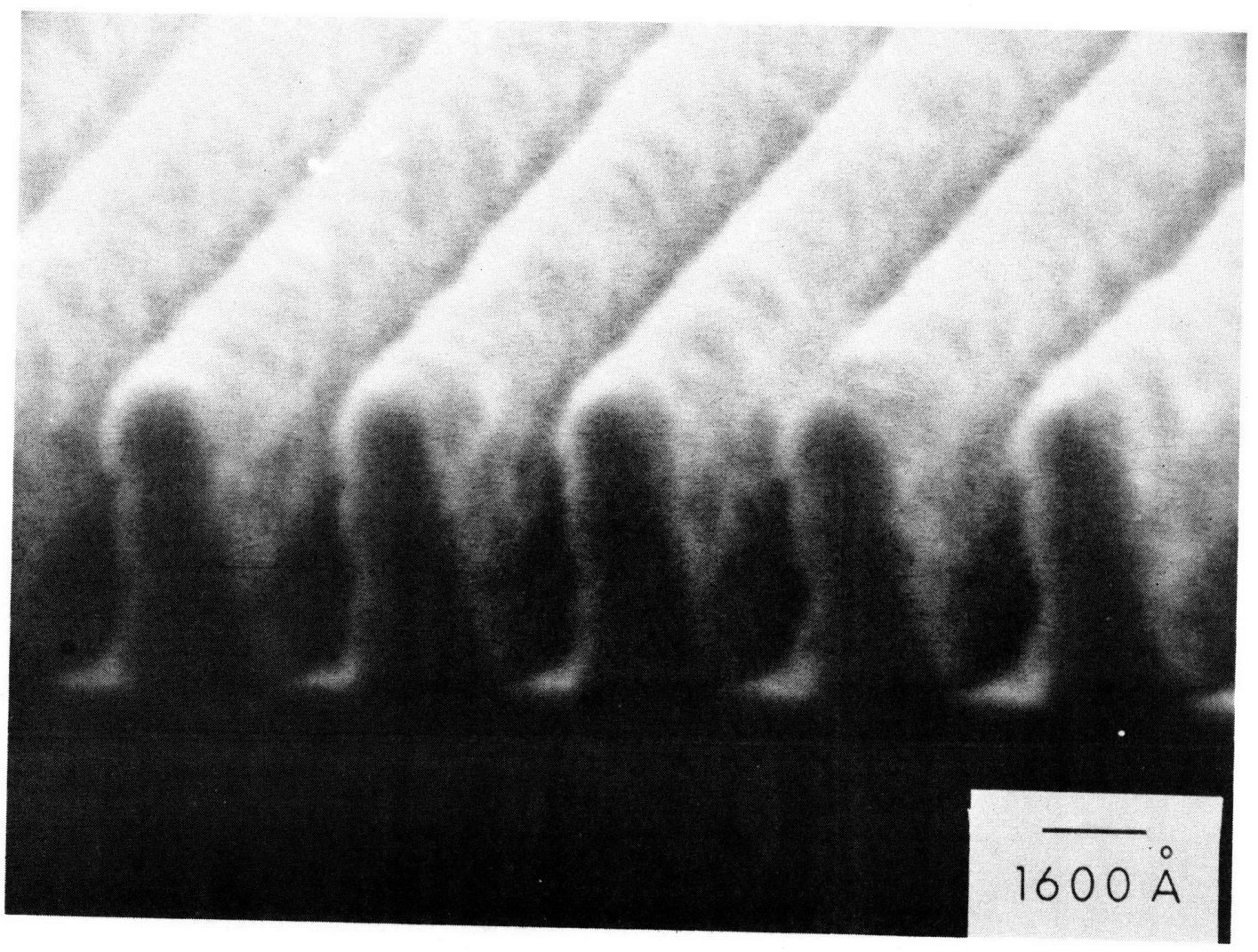

Fig. $3-4$. An SEM micrograph of a $320 \mathrm{~nm}(3200 \AA)$ period grating of Shipley $A Z$ - $1350 \mathrm{~J}$ photoresist on a glass substrate produced using simultaneous exposure and development holographic lithography. Note the roughness of
the sidewalls. 
The restriction to transparent substrates severely limits the range of substrate materials which can be used. The intrinsic rougness of the Shipley AZ-1350 photoresist which is quite evident in Fig. 3-2 and 3-4 appears to place a lower limit on the edge roughness which can be obtained directly with $A Z-1350$ resist. The poor adhesion of $A Z-1350$ resist to $\mathrm{SiO}_{2}$ also makes the process unreliable, since the photoresist can easily become detached from the substrate during normal processing. Because of the problems associated with the use of holographic lithography as a final lithographic step the use of the more flexible technique of X-ray lithography was explored. Details of this research are given in the next section. 


\subsection{PATTERN REPLICATION BY X-RAY LITHOGRAPHY}

\subsubsection{PROCESS CAPABILITIES}

Prior to this research $X$-ray lithography had been demonstrated to have several properties which made it particularly attractive as a final 1ithography step for the exposure of submicrometer surface relief patterns $^{4}$. These properties include:

i. Intrinsic high resolution because of the absence of significant diffraction at the 1-100 $\AA$ wavelengths which can be used.

ii. The compatibility of the extremely high resolution resist Polymethylmethacrylate (PMMA) with the process.

iii. The absence of back reflection problems, which makes possible the achievement of vertical sidewall resist structures.

iv. The relative simplicity of the technique and its low cost.

v. Reasonably short exposure time per sample. $(\approx 20-100 \mathrm{~min}$. for $\mathrm{Cu}_{\mathrm{L}}(13.3 \mathrm{~A})$ with a 400 watt electron bombardment source.) Despite these important properties, replication of square profiles with linewidths less than $500 \mathrm{~nm}$ had not yet been demonstrated. During this research the replication of very smooth-walled square cross-section profiles in PMMA with linewidths as small as $100 \mathrm{~nm}$ was achieved using the very soft $C_{L}(13.3 \AA)$ and $C_{K}(44.8 \AA) X$-rays. Details of this achievement are presented in this section. 


\subsubsection{CONSIDERATIONS IN THE CHOICE OF $\mathrm{Cu}_{L}$ and $C_{K} X$-RAYS FOR SUBMICROMETER PATTERN REPLICATION}

The X-ray lithography process using a conventional electron bombardment source is shown schematically in Fig. 3-5. An X-ray image is produced in the PMMA by modulating a uniform X-ray flux with a pattern of X-ray absorbing material carried on a relatively transparent mask membrane.

In general there will be a gap, $s$, between the mask and substrate. Since the $X$-ray source has a finite size and is not collimated, the shadow cast by the absorber pattern will exhibit penumbral blurring and geometric distortion. Both of these problems can be effectively eliminated by maintaining the mask and substrate in intimate contact and/or by increasing the source to substrate distance.

U1 timately the resolution of $\mathrm{X}$-ray lithography is limited by the instrinsic resolution of the $X$-ray sensitive resist. Therefore the resist characteristics are of utmost importance. The $X$-ray sensitive resist with the highest known resolution is PMMA. Its properties have been studied extensively be several workers $15,16,17$ and the mechanism of exposure and development is well understood. In this work, the unexposed resist consists of PMMA of a uniformly high molecular weight, $(950,000$ molecular weight PMMA was used). The exposing X-radiation breaks bonds in the long chain polymers, reducing the molecular weight in the exposed region. The resist is developed by immersion in a weak solvent which preferentially dissolves the low molecular weight material such as a 


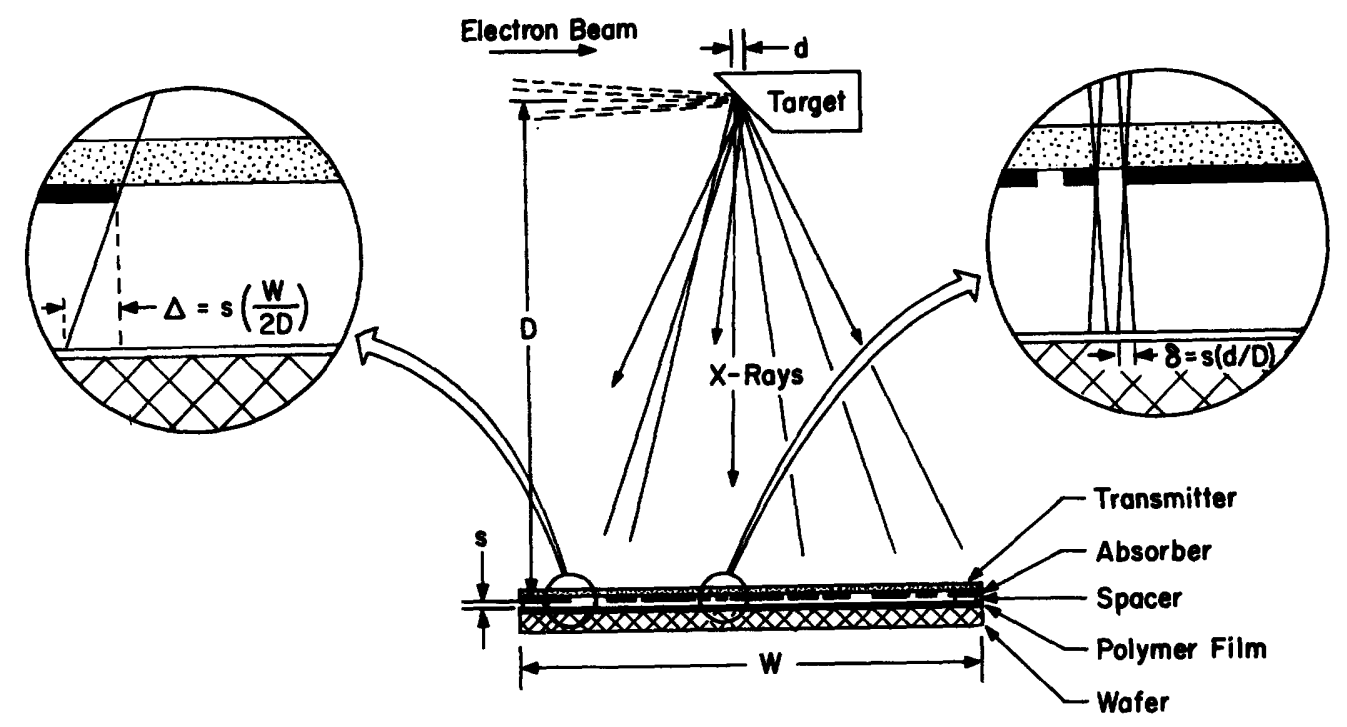

$X$-RAY LITHOGRAPHIC SCHEME

Fig. 3-5. I17ustrates the $x$-ray lithographic replication process using an eléctron bombardment $X$-ray source. An $X$-ray mask, consisting of an $X$-ray transparent membrane holding an absorber pattern, casts a shadow on the radiation sensitive polymer on the substrate. The insets show the effects of nenumbral blurring and geometric distortion of the replicated pattern caused by the finite source size, $d$, and noncollimated nature of the exposina radiation. 
mixture of $60 \%$ isoproply alcohol (IPA) and $40 \%$ Methyl Isobutyl Ketone (MIBK). The dissolution rate of PMMA for this mixture and ethyl alcohol and pure MIBK has been found to be a highly nonlinear function of the energy dissipated per unit volume in the PMMA $^{9}$. A plot of the measured dependence of dissolution rate on energy dissipation for electron exposure is shown in Fig. 3-6. (Similar results have been obtained by Bernacki ${ }^{18}$ for $\mathrm{Al}_{k}$ X-ray exposure.)

In the case of $X$-ray lithography the resolution of PMMA appears to be limited only by the range of the photoelectrons emitted upon absorption of an $\mathrm{X}$-ray photon. Figure 3-7 shows a calculation ${ }^{19}$ of the maximum range of photoelectrons as a function of energy. Also shown is a curve extrapolated from measurements of Spiller's ${ }^{20}$ of an effective electron range in PMMA, a measure of the distance over which a significant exposure occurs. Note that the effective range is $400 \AA, 200 \AA$, and $50 \AA$ at $A 1_{k}, 8.3 \AA$, $C u_{L}(13.3 \AA)$, and $C_{k}(44.8 \AA)$ respectively. This indicates that the longer $X$-ray wavelengths are more desirable for the highest resolution work, than the $\mathrm{Al}_{k} \mathrm{X}$-ray which is commonly used for linewidths of $>0.5 \mu \mathrm{m}$.

The choice of the optimum $X$-ray wavelength for replicating $\approx 1000 \AA$ linewidth grating patterns with smooth vertical sidewalls is determined by several considerations.

i. The absorber attenuation must be $>5 \mathrm{db}$ to provide adequate contrast for optimum exposure.

ii. In general the gold absorber pattern is not perfect and has considerable high spatial frequency edge roughness and some edge smoothing is desirable. 


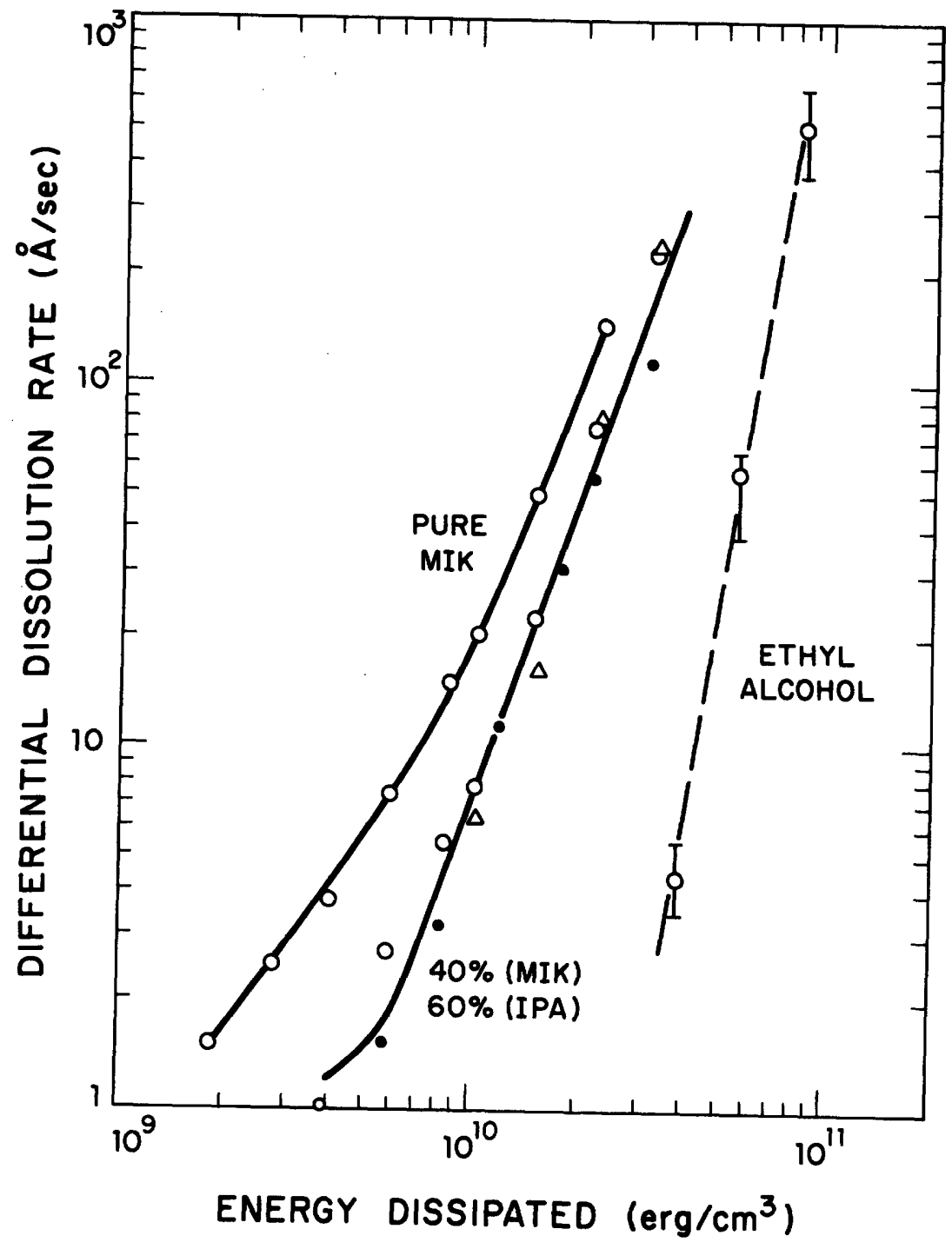

Fin. 3-6. Differential dissolution rate of a $\sim 0.3 \mu \mathrm{m}$ thick film of PIMA as a function of eneray dissipated in the polymer for three different solvents: ethyl alcohol, a solution of $40 \%$ (MIK) and $60 \%$ (IPA), and pure MIK. (From reference (9)) 


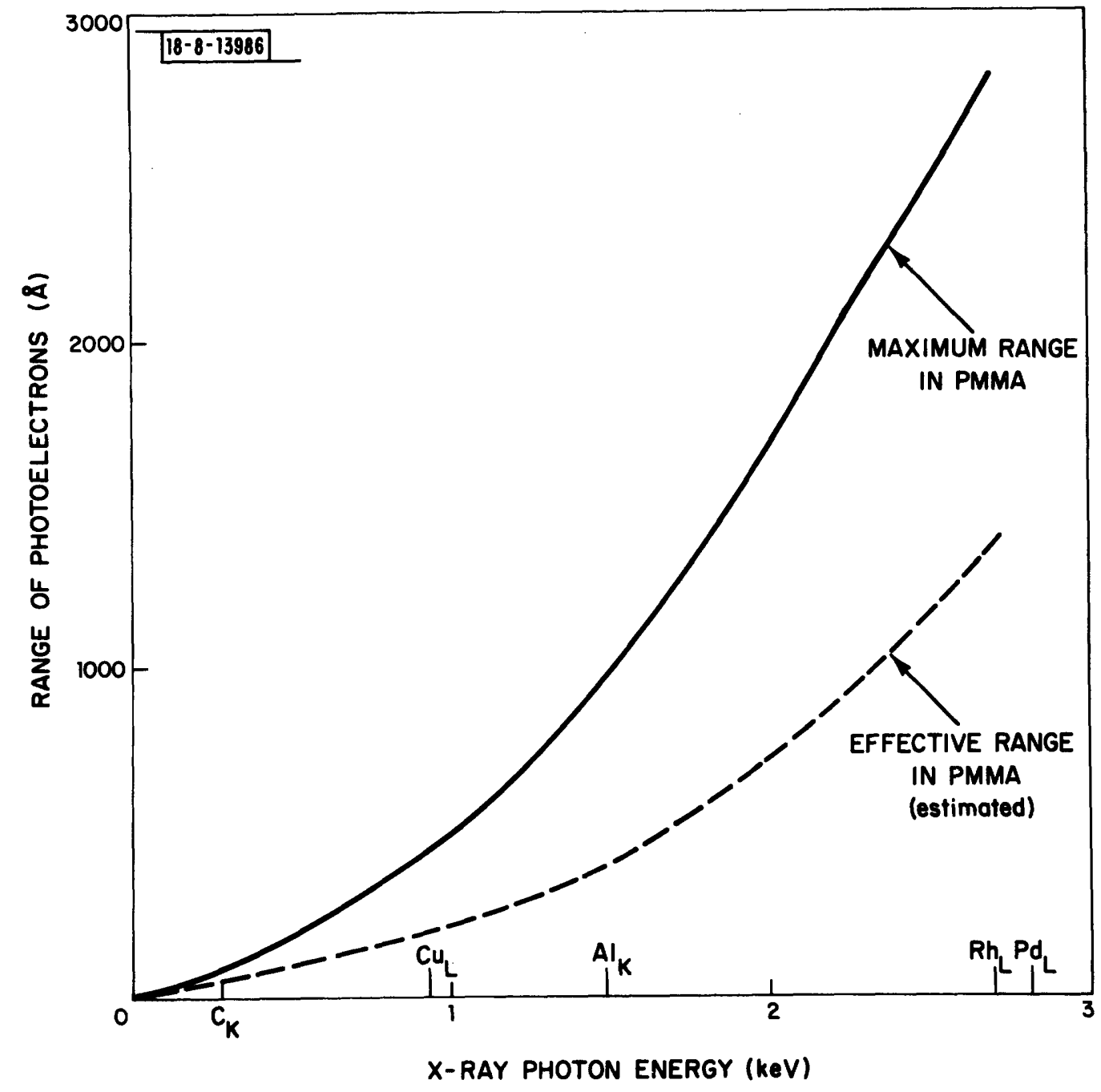

Fic. 3-7. The calculated maximum rance of photo ${ }^{19}$ electrons in PI:MA yersus electron eneray. Shown on the same plot is a curve extrapolated from data obtained by Spiller of the effective range of photo electrons, the rance over which sianificant exposure of the PMAA occurs. 
iii. A mask membrane material with reasonably low attenuation must be available.

iv. An X-ray source with a strong $X$-ray line must be available. It has been found that the $13.3 \AA \mathrm{C} \mathrm{L}_{L} X$-ray is nearly optimum given the above constraints. The reasons for this are outlined below:

Figure 3-8 shows a plot of the attenuation in gold and polyimide versus $X$-ray wavelength ${ }^{21,22,29}$. Gold is generally the material chosen for an X-ray absorber. It exhibits one of the highest material absorptions of any element and is a very convenient material to use in mask pattern fabrication because of its high sputter etching rate. Nevertheless fabrication of gold structures with aspect ratios much greater than one by ion beam etching is difficult. Therefore, if replication of linewidths less than $100 \mathrm{~nm}$ is desired, reference to Fig. 3-8 indicates that the $X$-ray wavelength used should be greater than $\approx 12 \AA$ in order that the pattern will have contrast $>5 \mathrm{db}$. At the same time, because the edges of the grating pattern are rough, some blurring or smoothing of the replicated pattern is desirable. For this reason using the shortest practical wavelength is advantageous since photoelectron range increases with decreasing wavelength.

The attenuation of most materials increases rapidly with wavelength above $10 \AA$. To minimize absorption and maximize film thickness for reasons of strength and stability, the mask membrane should have a low material attenuation. Fortunately several organic polymers which are particularly convenient to use in thin film form as mask membranes have 


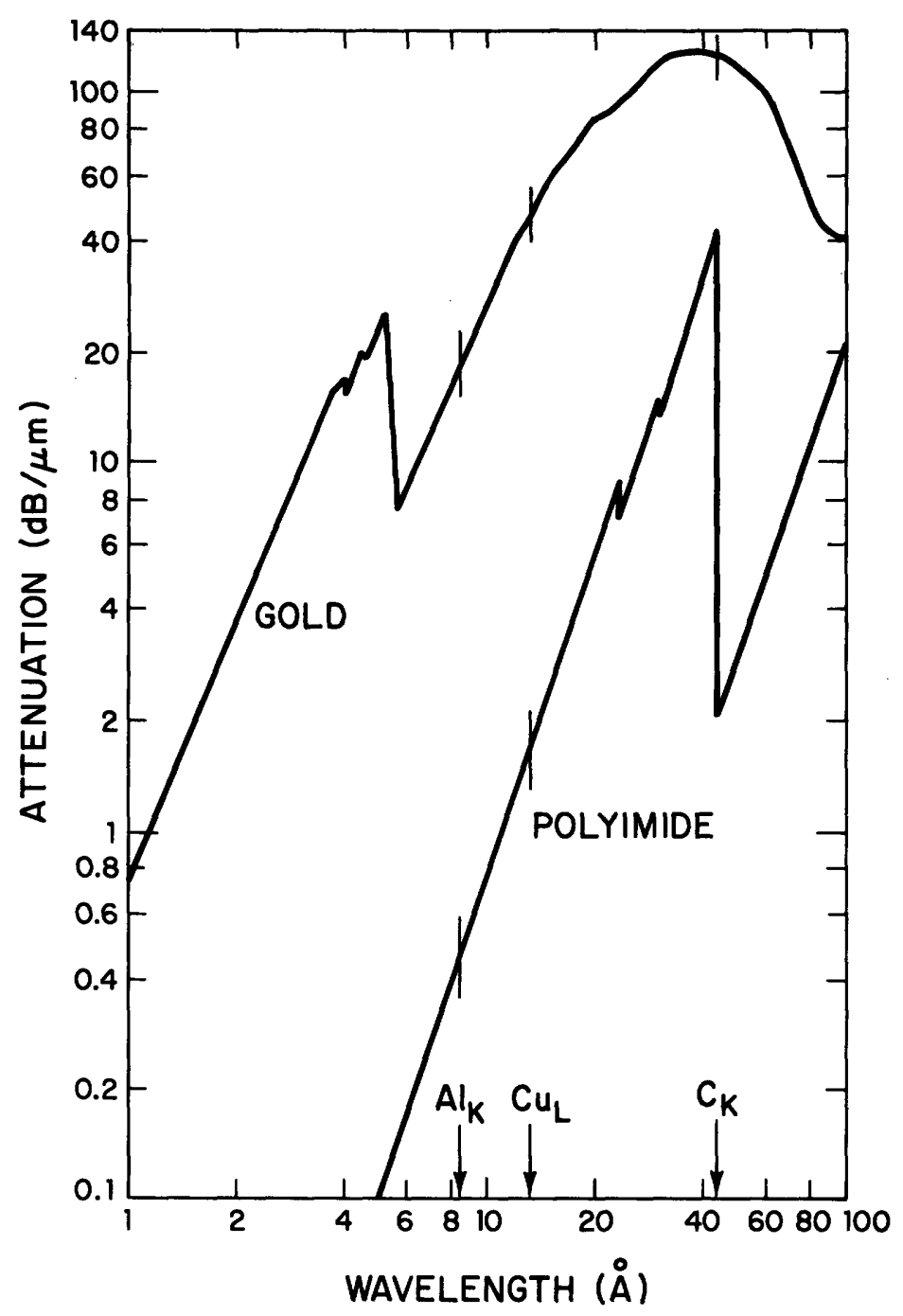

Fic. 3-8. A plot of the attenuation ( $\mathrm{gb} / \mathrm{um}$ ) of gold and polyimide versus wavelenath for soft $x$-rays $(1-100 \AA)$. The data was obtained from several sources. 21,22,29 
acceptable attenuations of $<3 \mathrm{db} / \mu \mathrm{m}$ for wavelengths below $15 \AA$ and in the region of the carbon absorption edge at $44.8 \AA$. The attenuation curve of polyimide shown in Fig. 3.8 is typical of many high carbon content polymers. Lastly, copper which exhibits a strong $L$ line at $13.3 \AA$ is a convenient material to use as an electron bombardment source because of its high thermal conductivity and relatively high melting point.

The $44.8 \AA C_{k} X$-ray is also very well suited for high resolution $X$-ray 1 ithography ${ }^{20}$. In fact it appears to be the optimum wavelength for the highest resolution work because the maximum attenuation of gold occurs near $44.8 \AA$ and low attenuation mask membrane materials are available at $44.8 \AA$ because of the occurance of the carbon absorption edge. For wavelengths greater than $50 \AA$ the resolution limit is probably determined by diffraction rather than photoelectron range. If better absorber patterns can be made and the edge smoothing effect of the $\mathrm{Cu}_{L}$ radiation is not necessary the $C_{k} X$-ray is expected to be the optimum wavelength for high resolution work. 


\subsubsection{THE X-RAY SOURCE}

A schematic of the apparatus which was developed to implement the $X$-ray lithographic process is shown in Fig. 3-9. X-rays are generated by electron bombardment of a copper or carbon target. Any electrons escaping from the $X$-ray source are deflected by an electron deflection assembly and thus prevented from striking the substrate. The PMMA coated substrate is mounted in thermal and electrical contact with a water cooled copper block. The X-ray mask is maintained in intimate contact with the substrate by an electrostatic hold down scheme.

The electron bombardment X-ray source consists of a modified Vacuum Generators EG-1 electron beam evaporation unit ${ }^{24}$. Electrons emitted from the resistance heated tungsten ring cathode are electrostatically focused to an $\approx 1 \mathrm{~mm}$ diameter spot in the center of the water cooled anode. The anode consisted either of a $2 \mathrm{~mm}$ thick copper disk or sandwich of $1 \mathrm{~mm}$ thick high density graphite, $250 \mu \mathrm{m}$ thick indium, and $1 \mathrm{~mm}$ thick copper. It was determined experimentally that the copper and carbon anodes would begin to evaporate at total power levels of $\approx 550$ watts into an $\approx 1 \mathrm{~mm}$ spot. In practice a maximum total power dissipation of 400 watts was used.

Early X-ray exposures showed much less contrast than predicted. This problem was traced to spurious PMMA exposure from stray electrons emitted from the electron gun assembly. This problem was eliminated by installing an electron deflector assembly above the X-ray source, (See Fig. 3-9).

The $X$-radiation emitted by the electron bombardment source consists of line radiation and continuum or bremsstrahlung radiation. The wave- 


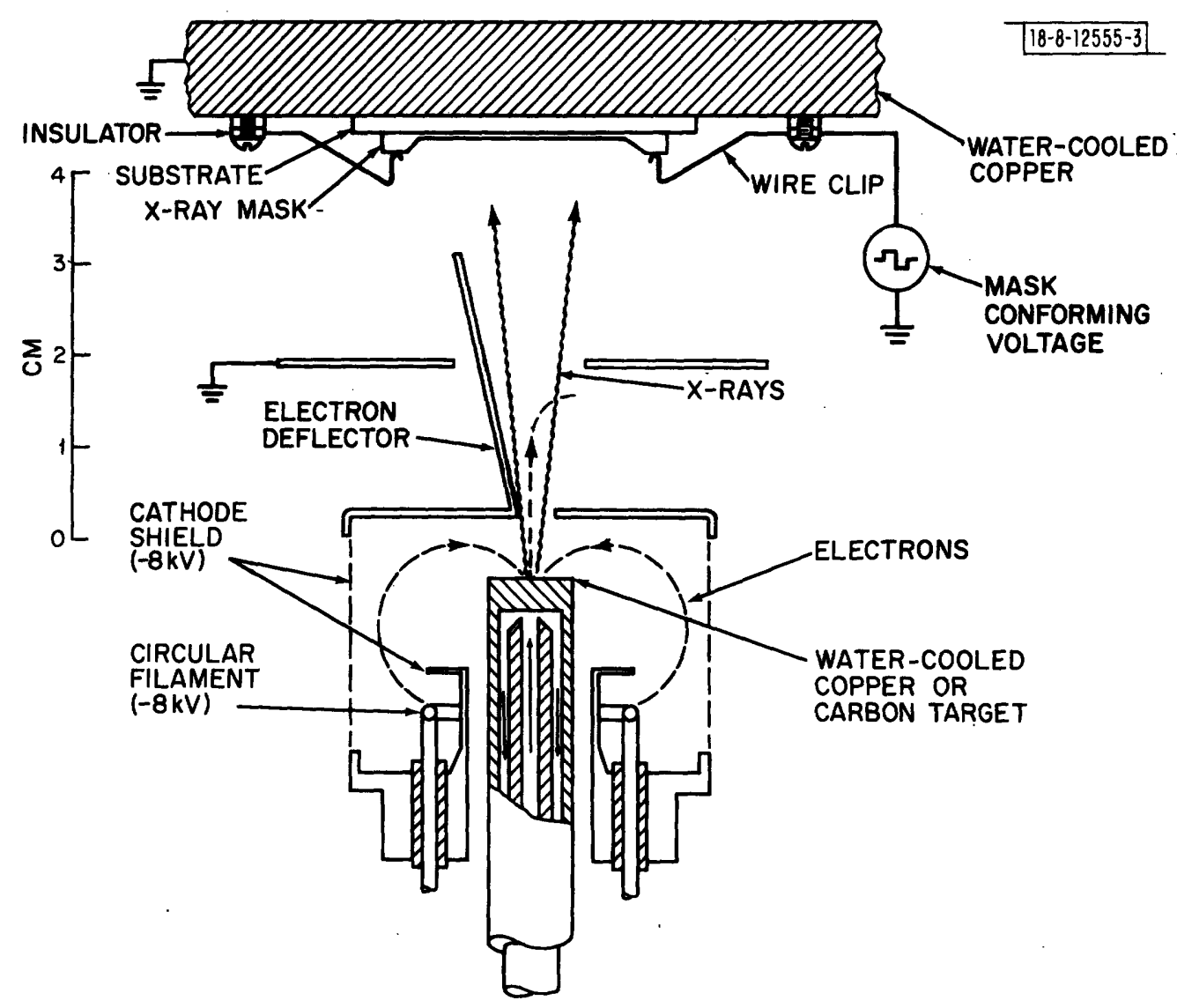

Fig. 3-9. A schematic diagram of the apparatus used to implement $X$-ray lithography with the $\mathrm{Cu}_{1}$ and $C_{k} X$-rays. The dimensions and voltages shown are typical operating parameters. A source to substrate distance of $7.5 \mathrm{~cm}$, and accelerating voltage of $6.5 \mathrm{kv}$ were also commonly used. 
length of the desired line radiation is of course determined by the target material. The spectrum of the continuum is determined by the material and the energy of the incident electron, with the upper limit of the photon energy equal to the incident electron energy.

The following empirical expressions ${ }^{25}$ indicate the dependence of $x$-ray line and continuum emission on material parameters and electron energy.

The number of photons emitted, $N$, per steradian, per electron is given by

$$
\frac{N}{4 \pi}=\frac{1}{4 \pi} \quad \text { f.e }\left(E_{e}-E x\right)^{1.63}
$$

The power in the continuum in total watts emitted per KeV interval per watt of electrical power is given by

$$
P\left(E_{p}\right)=k \cdot f\left(E_{p}\right) \cdot Z\left(1 \frac{-E_{p}}{E_{e}}\right)
$$

where $\varepsilon$ is characteristic of the target element, $f$ is the target reabsorption factor, $E_{e}$ is the energy of the bombarding electrons in KeV, $E_{X}$ is the ionization energy of the shell ( $K$ or $L$ ) of interest in KeV, $E_{p}$ is the photon energy in KeV, Z, is the atomic number of the substrate, and $k$, is a number characteristic of the material.

of primary importance is the relative power absorbed in the PMMA from the line and continuum radiation since the mask will have low contrast for the higher energy continuum radiation. Using equations 3.3 and 3.4 and the measured ${ }^{29}$ absorption versus wavelength of PMMA the total power in watts/watt/steradian absorbed in 1 um of PMMA per unit photon energy was 
calculated for a copper source operated at $8 \mathrm{kV}$ (just below the threshold for excitation of the $C u_{k} 1.5 \AA$ line). The result is plotted below in Fig. 3-10. The total power absorbed from the continuum is $\approx 5 \%$ of the power absorbed from the line radiation. Because of the high nonlinearity of the resist development rate as shown in Fig. 3-6 the effect of this additional exposure by the continuum should be negligible, (provided that the mask membrane does not significantly alter the spectrum of the exposing radiation). 


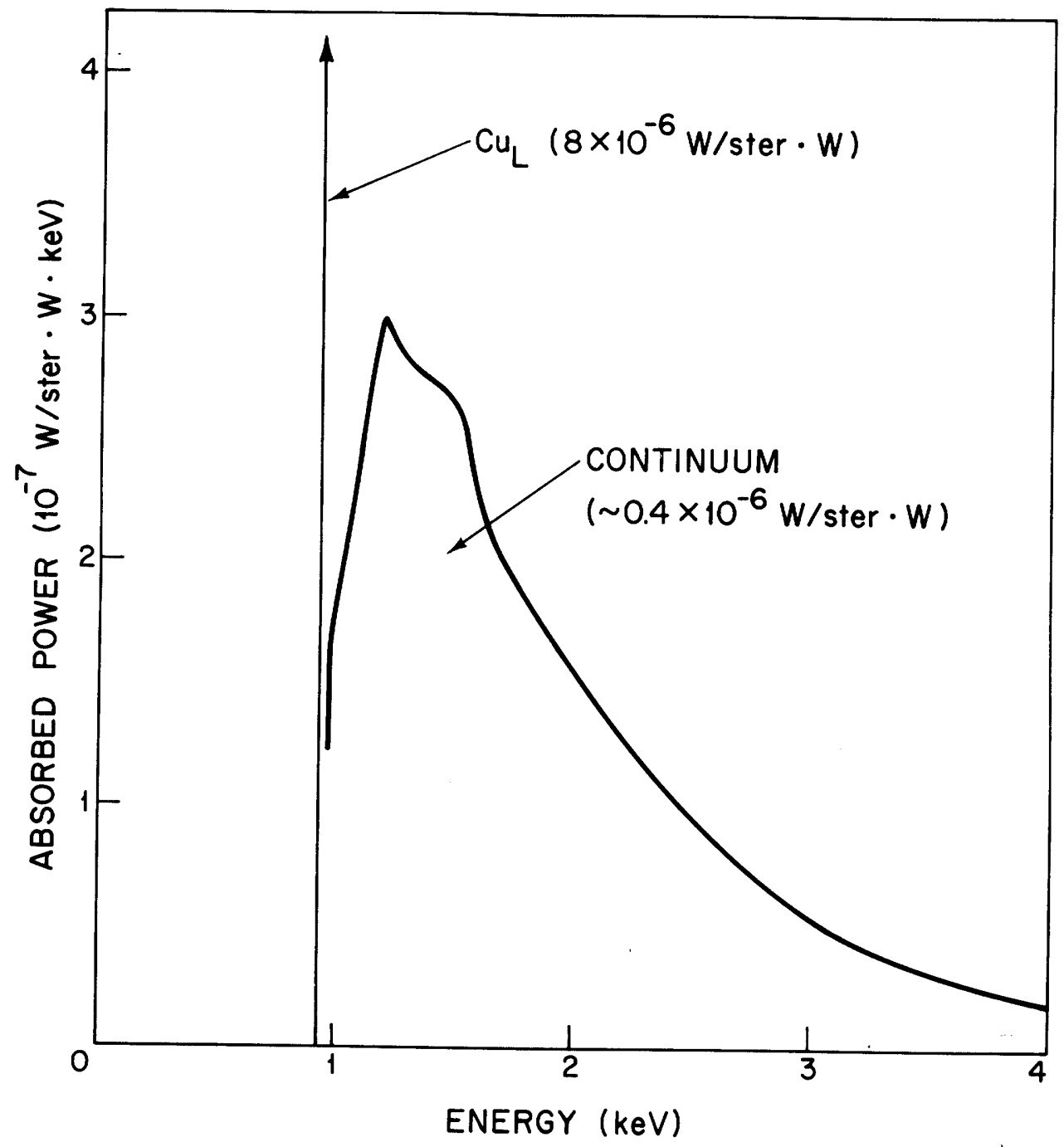

Fia. 3-10. The calculated X-ray power from a copper X-ray source operating at $-8 \mathrm{kV}$ absorbed in $1 \mu \mathrm{m}$ of PIMAA (the units are, power absorbed in watts, per steradian, per watt of pover dissipated in the conner taraet, ner Kev interval of photon eneray). 


\subsubsection{THE FABRICATION OF X-RAY MASKS}

A major problem encountered in implementation of X-ray lithography with the $\mathrm{Cu}_{L}$ and $\mathrm{C}_{k} X$-rays was the fabrication of the $X$-ray mask. Several techniques had been developed by other researchers $26,27,28$ to produce masks for use with the $8.3 \AA \mathrm{Al}_{k} \mathrm{X}$-ray, and other shorter wavelength $X$-rays. However, the attenuation in the mask membrane of these masks at the $\mathrm{Cu}_{\mathrm{L}}$ and $\mathrm{C}_{k}$ wavelengths were unacceptably high (i.e., $>3 \mathrm{db}$ ). Membranes used at the shorter wavelengths are usually a few um thick while material attenuation at wavelengths longer than $\approx 12 \AA$ forces one to use thicknesses $1 \mu \mathrm{m}$ or less.

To solve this problem, several new types of mask membranes were investigated, including, very thin $(\approx 200 \mathrm{~nm}) \mathrm{Si}_{3} \mathrm{~N}_{4} / \mathrm{SiO}_{2}$ membranes, and various thin organic polymer films including mylar, polethylhene, and polyimide. Useful X-ray masks were fabricated with silicon nitride using a process similar to that developed by Spiller 29,30 . These masks were found to be extremely fragile and their use was abandoned. Nevertheless, the technology developed to fabricate silicon nitride masks has been very useful in another part of the thesis work, namely, sample preparation prior to observation in transmission electron microscopy as described in section 3.5. The fabrication of silicon nitride $X$-ray masks is detailed in Appendix A. Commercial Mylar film which has been used as a mask membrane with the $A 1_{k} X$-ray and shorter wavelengths ${ }^{27,28}$ was found to have an extremely rough surface unsuitable for high resolution. work. Polyethlylene films which can be easily prepared in $0.5 \mu \mathrm{m}$ thicknesses by 
stretching a $25 \mu \mathrm{m}$ thick sheet were found to be extremely heat sensitive and could not withstand ion-beam etching of the gold mask pattern. Eventually this research led to a very successful new process for fabricating $X$-ray masks with polyimide mask membranes ${ }^{31}$. This new mask was used for nearly all the soft X-ray lithography work presented here. The process developed for fabricating polyimide $X$-ray masks is shown schematically in Figures $3-11$ and 3-12. In the first step of the process, a glass substrate is coated with a film of polyamic acid (Dupont product PI-2530) $)^{32}$ using a conventional spinning technique. Both $200 \mu \mathrm{m}$ thick Corning $0211 \mathrm{glass}^{33}$ substrates and $1.5 \mathrm{~mm}$ thick optically flat glass substrates were used. Polyamic acid thicknesses ranging from $0.5 \mu \mathrm{m}$ to $5 \mu \mathrm{m}$ were obtained by varying the spinning speed and the dilution of the polyamic acid in a solution of equal parts of $\mathrm{N}$-Methyl-2-pyrollidone and acetone. To obtain the film thickness of $0.9 \mu \mathrm{m}$ used for most of the $\mathrm{Cu}_{\mathrm{L}}$ work a mixture of 4 parts PI-2530, 1 part N-Methyl-2-pyrollidone, and 1 part acetone was spun at $6000 \mathrm{rpm}$ for $60 \mathrm{sec}$. After spinning, the polyamic acid is converted to polyimide by curing the film at $150{ }^{\circ} \mathrm{C}$ for 15 minutes and then at $250^{\circ} \mathrm{C}$ for 60 minutes. Next the absorber pattern is fabricated. A layer of $10 \mathrm{~nm}$ of chrome (for adhesion) and a layer of $100 \mathrm{~nm}$ of gold are evaporated onto the polyimide surface, then $100 \mathrm{~nm}$ of $A Z-1350 \mathrm{~J}$ resist is spun onto the gold surface (2:7, AZ-1350J: AZ-thinner at $5000 \mathrm{rpm})$. A grating pattern is exposed in the resist using the holographic technique described in section 3.2. This resist grating pattern then acts as a mask during ion-beam etching ${ }^{4}, 36$ of the gold. After 

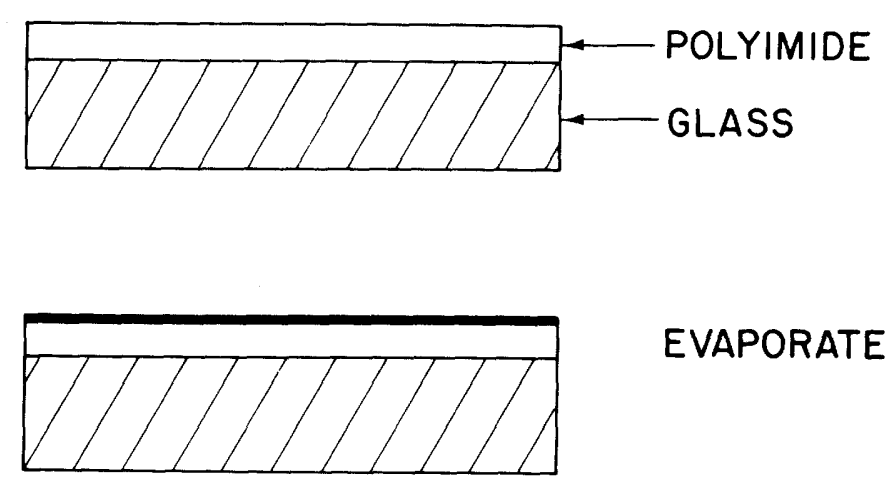

EVAPORATE GOLD

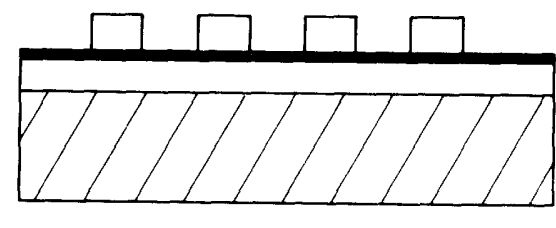

\author{
EXPOSE RESIST
}

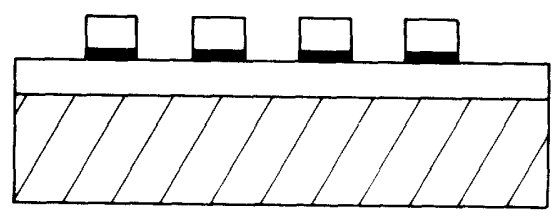

ION BEAM ETCH

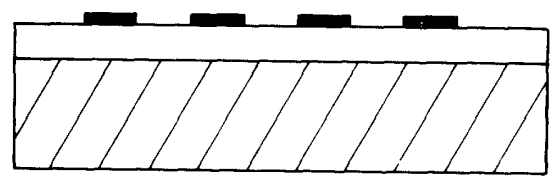

REMOVE RESIST

Fig. 3-11. The processing sequence for fabricating polyimide membrane $X$-ray masks with gold absorber patterns. 

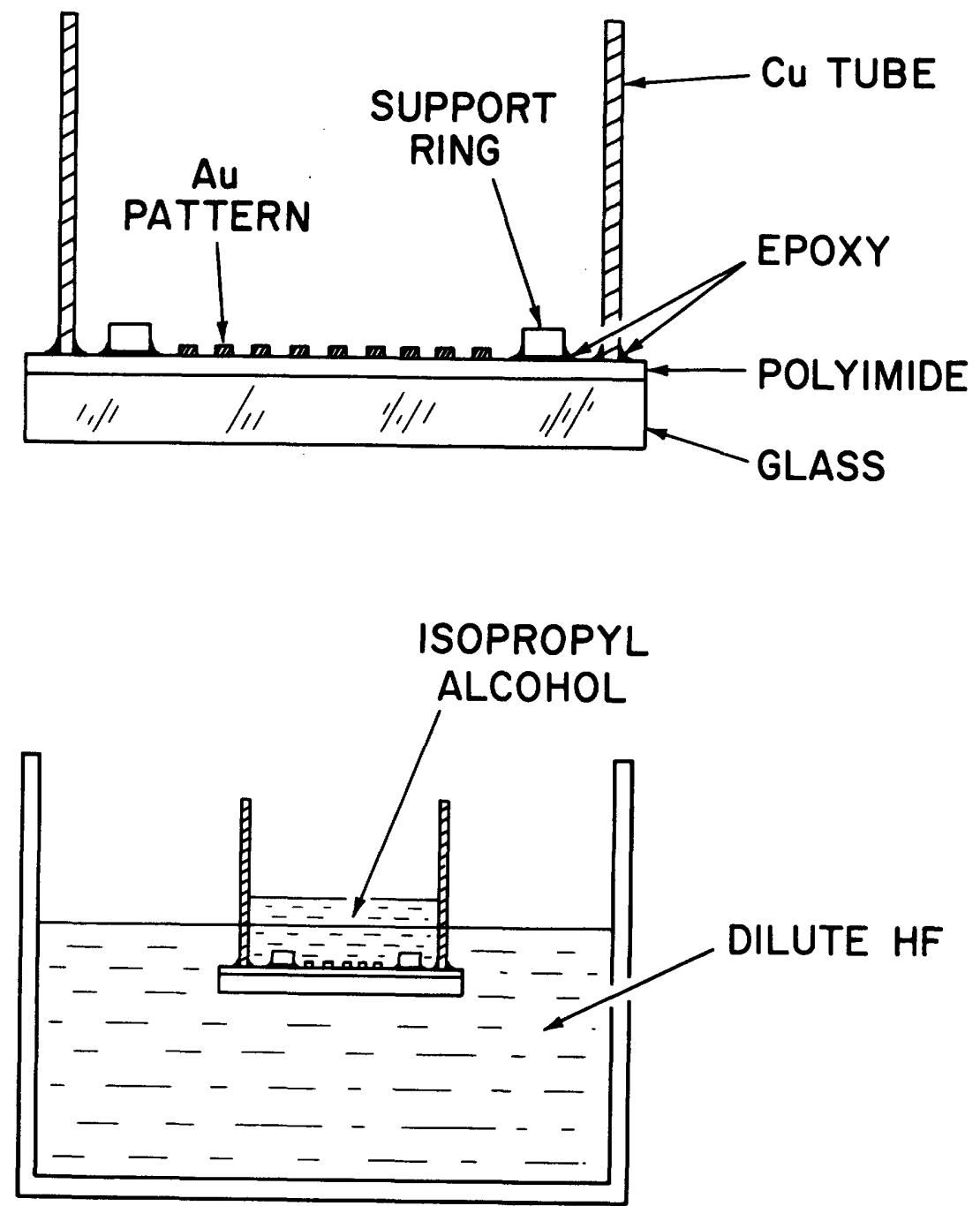

Fia. 3-12. a). A schematic cross-secti in is shown of the tonding of a conner tube etching holder to a polyimide film prior to etching of the alass substrate. An optional step of bonding of a support ring is also shown. b). The method of removina the alass substrate by etching in dilute hydroflouric acid is illustrated. Isopropyl alcohol can be used as shown to prevent any acid from leaking through pinholes in the nolvimide which may be present when the glass has been removed. 
ion-beam etching of the gold a copper tube is bonded to the polyimide using epoxy ${ }^{34}$ as indicated in Fig. 3-12(a). (At this point a support ring can also be bonded to the polyimide.) The glass substrate is etched away by immersing the assembly in a solution of 2 parts water to 1 part concentrated hydroflouric acid. Following removal of the glass a beveled support ring of aluminum or stainless steel is bonded to either side of the polyimide membrane using epoxy ${ }^{35}$, (unless it was already done before the glass was removed). After the membrane has been mounted on a support ring a layer of $50 \mathrm{~nm}$ of aluminum is evaporated over the absorber side of the mask membrane. Electrical contact is made between the aluminum layer and the support ring using conductive paint or by insuring a continuous path between the aluminum and the ring. Cross-sections of the completed polyimide $X$-ray mask configurations which result are shown in Figures 3-13 and 3-14.

Further details about the fabrication of polyimide X-ray masks are given in Appendix $B$. 


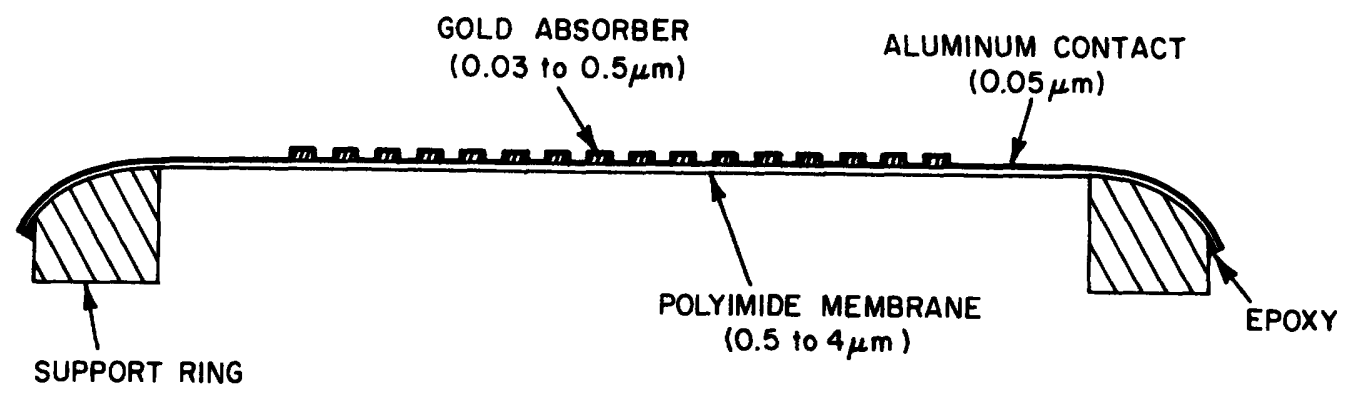

Fig. 3-13. Schematic cross-section of a polyimide $X$-ray mask developed for hiah resolution $\mathrm{X}$-ray lithoaraphy using the $\mathrm{Cu}_{L}$ and $\mathrm{C}_{k} \mathrm{x}$-ray wavelencths. Here the gold absorber pattern is on the "front side of the mask. 


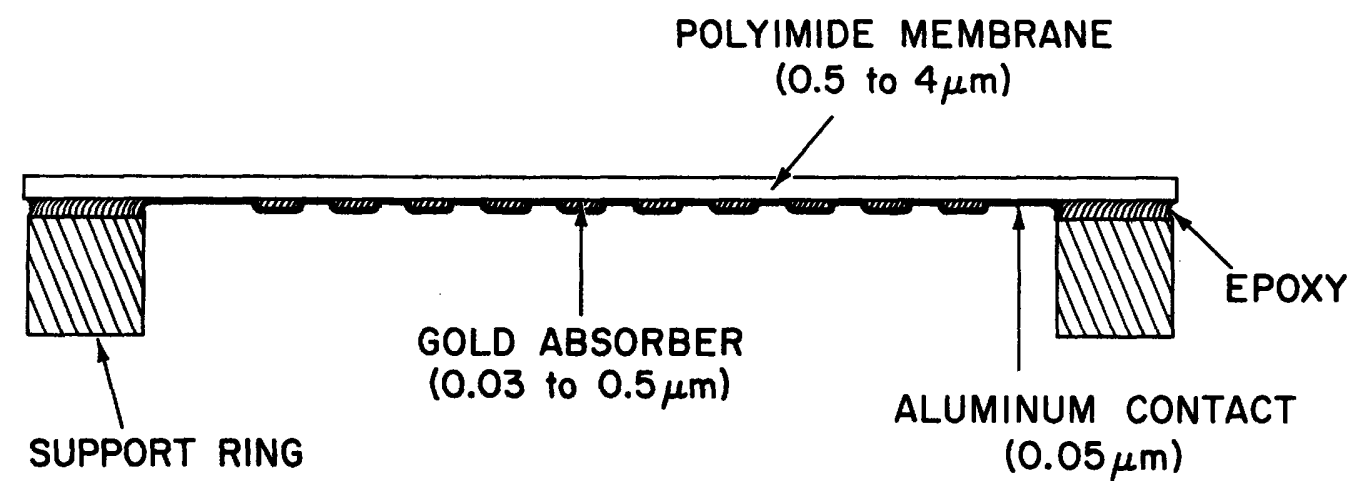

Fig. 3-14. Cross-sections of two types of polyimide X-ray masks are shown where the gold absorber pattern in on the "back" of the mask.

a.) The configuration which results when a flat support ring is bonded to the polyimide before etching, as shown in Fig. 3-12. 


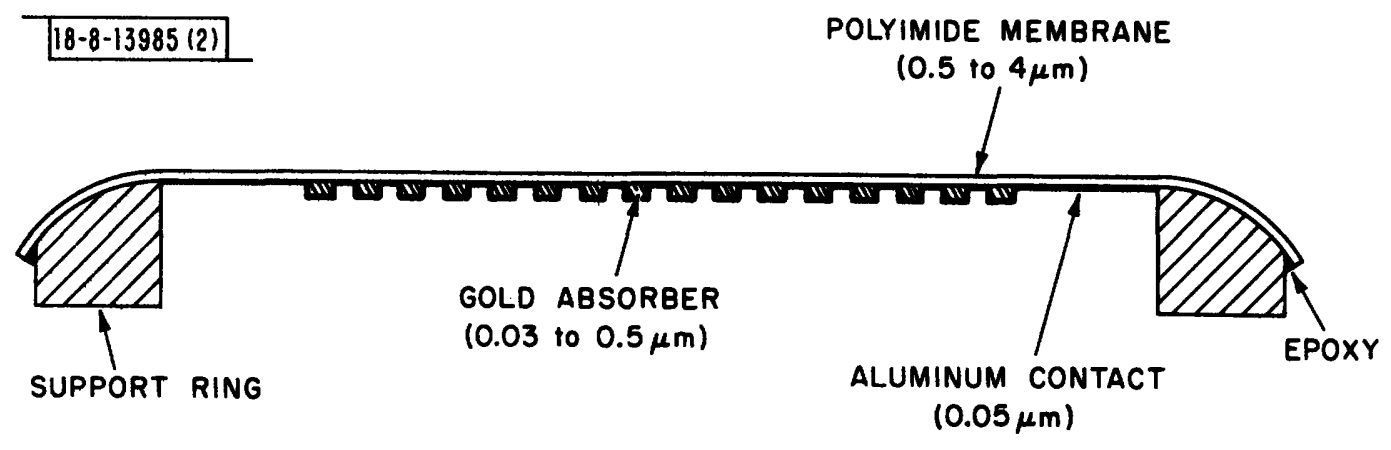

Fig. 3-14. Cross-sections of two types of polyimide X-ray masks are shown where the gold absorber pattern in on the "back" of the mask.

b.) The configuration which results when a beveled support ring is bonded after etching of the substrate. 


\subsubsection{THE MAINTENANCE OF INTIMATE MASK-SUBSTRATE CONTACT}

To minimize penumbral blurring during $X$-ray exposure the mask and substrate are held in intimate contact. Since the exposure takes place in a vacuum the usual pneumatic hold down methods cannot be used. For this reason an electrostatic hold-down technique was developed which employs the force developed across a parallel plate capacitor when a voltage is applied. Three distinct exposure geometries have been used in this work as shown in Fig. 3-15.

Given the insulator thickness, $t$, in $\mu \mathrm{m}$ and dielectric constant, $k_{c}$, of the insulator, the pressure, $P$, in atmospheres exerted upon the $X$-ray membrane will be

$$
P=\left(4.4 \times 10^{-5}\right) \quad k_{c} \frac{v^{2}}{t^{2}}
$$

For typical values of $t=1 \mu \mathrm{m}, V=40$ volts, and $k_{c}=10$, the pressure, $P=0.7$ atmospheres, is more than adequate to maintain intimate contact. The integrity of the insulator between the mask and substrate conductors is essential to the maintenance of reliable contact since a short circuit will eliminate the hold down force. PMMA has been found to be an unreliable insulator because it is easily scratched and often contains pinholes, thus, either the substrate itself or the polyimide membrane must act as the insulator.

It was found that during exposure to $X$-rays a fixed charge tends to accumulate in the insulator such as to cancel the field between the 


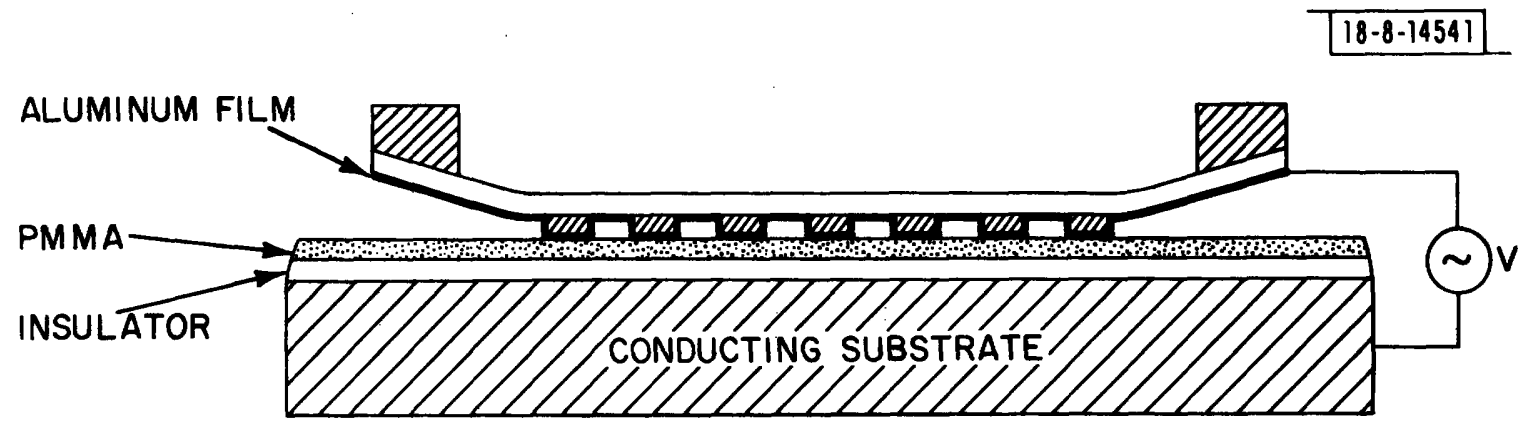

(a)

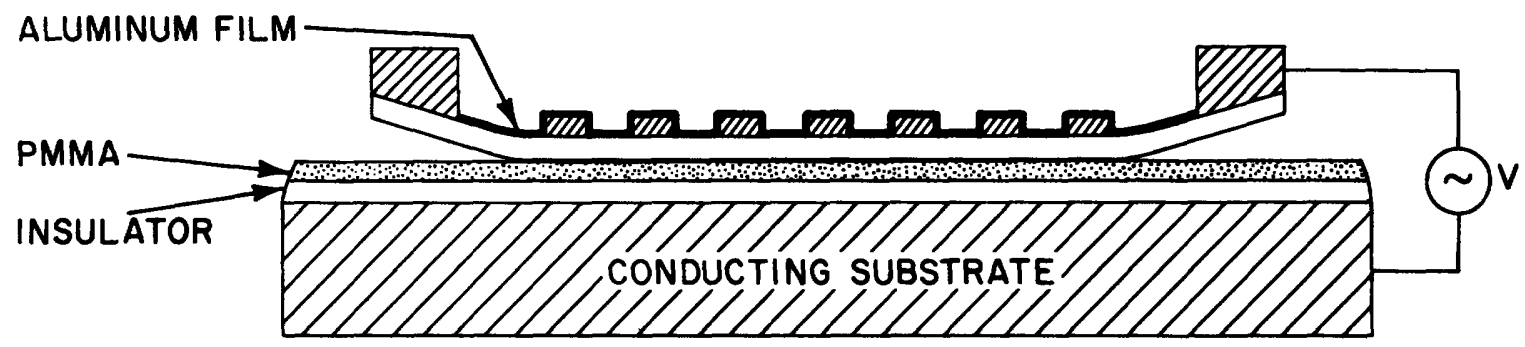

(b)

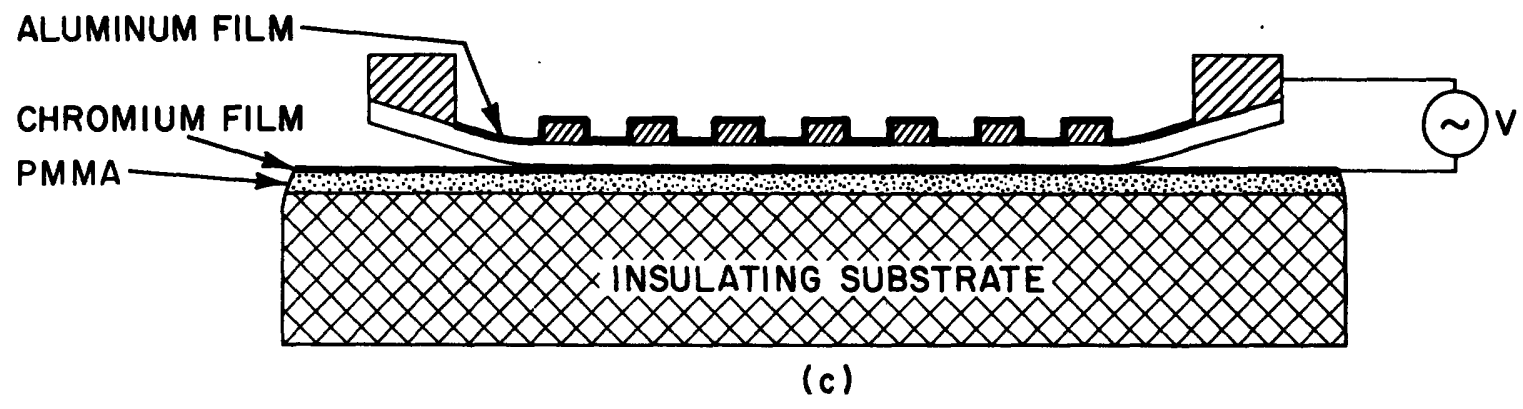

Fig. 3-15. Schematic cross-sections are shown of three mask and substrate geometries used to obtain intimate contact between a mask and substrate using electrostatic pressure. 
mask and substrate. If this process is allowed to continue the mask and substrate eventually lose contact. However, contact can be maintained by reversing the voltage across the mask and substrate before the field is completely cancelled. An automatic monitoring system was developed for this purpose. The area of contact of the mask and substrate is monitored by measuring the capacitance of the mask substrate combination. Before X-ray exposure, at a given voltage, the balance of electrostatic pressure and mask tension determine the area of contact. As the electrostatic pressure decreases, due to charging, the area of contact also decreases. At a preset threshold value of capacitance the sign of the contact voltage is automatically reversed and (at least) the intital contact area is regained. This process then repeats as long as the $x$-ray flux is incident on the mask. Continuous intimate contact is thus maintained. 


\subsubsection{EXPERIMENTAL RESULTS - PMMA PROFILES OBTAINED USING $C u_{L}$ AND $c_{k} X$-RAYS}

To test the X-ray replication process grating patterns of $320 \mathrm{~nm}$ period were replicated in PMMA layers $0.5 \mu \mathrm{m}$ and $1 \mu \mathrm{m}$ thick. SEM micrographs of the results are shown in Figures 3-16 to 3-20.

All of the exposures shown were done on $500 \mathrm{~nm}$ thick $\mathrm{SiO}_{2}$ which was thermally grown on silicon wafers. PMMA was spun onto the $\mathrm{SiO}_{2}$ as described in Appendix C. A polyimide X-ray mask of the type shown in Fig. 3-13 was used. The polyimide thickness was $\approx 0.9 \mu \mathrm{m}$ and the gold absorber thickness was $100 \mathrm{~nm}$. The exposure configuration shown in Fig. 3-9 was used where the source to substrate distance was $5 \mathrm{~cm}$ and a power of 400 watts was used. Switching of the voltage applied between the mask and substrate was employed to maintain mask-substrate contact. Exposure time under these conditions was $75 \mathrm{~min}$. The development rate (which decreased slowly with depth because of absorption of the X-rays) averaged $3.5 \mathrm{~nm} / \mathrm{sec}$. Development was monitored by observing the PMMA thickness in a fully exposed area adjacent to the grating. When the PMMA was completely removed in that area the development was terminated.

To observe the structures in the SEM the profiles, which are insulators, must be coated with a conducting film to prevent charging by the electron beam. This was done by RF sputtering of gold. It was found that the PMMA profiles could easily be distorted by heating during the gold sputtering. To minimize this effect the sputtering. was done at a 


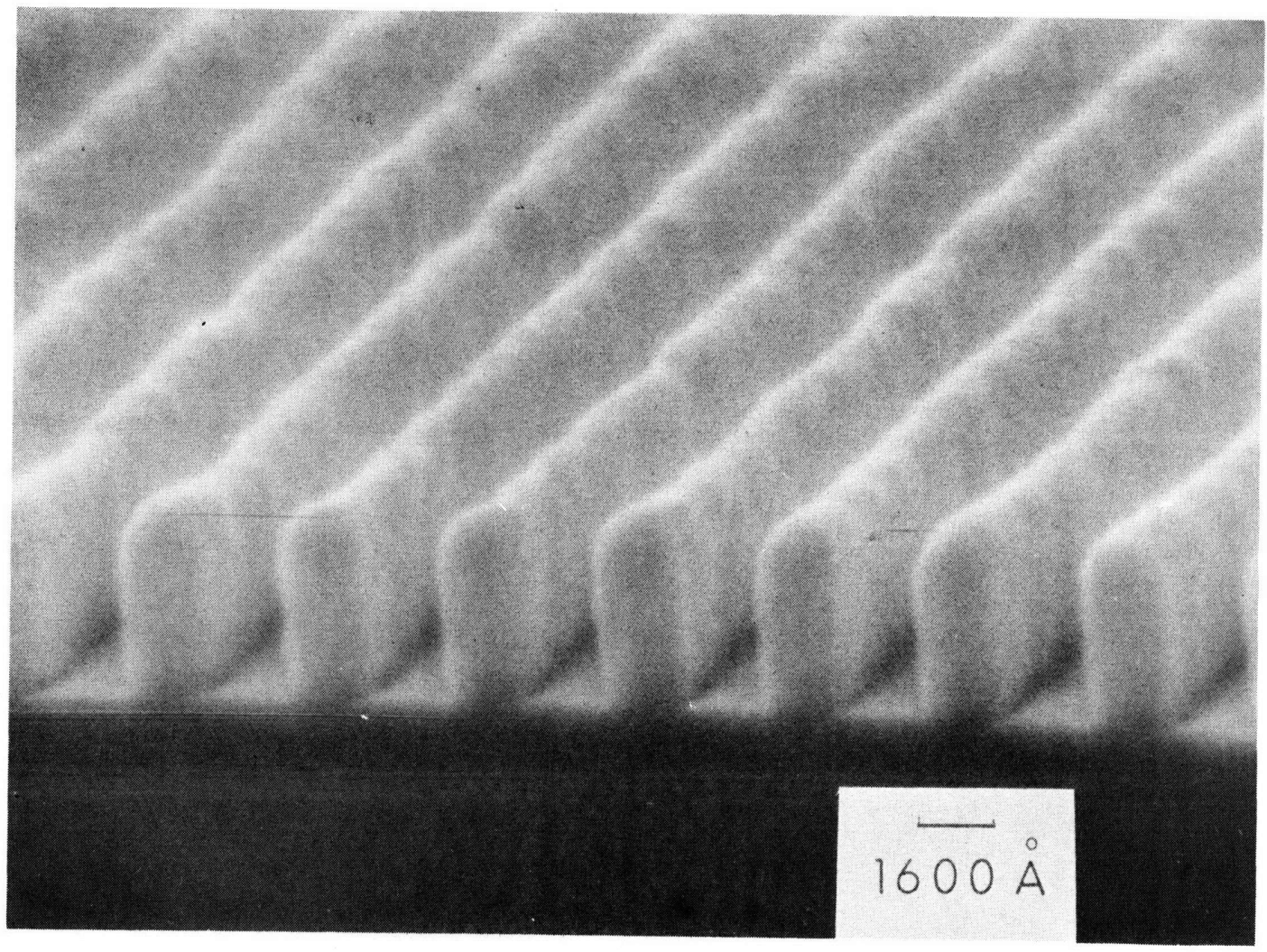

Fig. 3-16. SEM micrograph of a $320 \mathrm{~nm}(3200 \AA)$ period grating of PMMA on an amorphous $\mathrm{SiO}_{2}$ substrate which was exposed using the $\mathrm{Cu}_{\mathrm{L}}(13.3 \AA) \mathrm{X}$-ray. The depth of the grating is $400 \mathrm{~nm}$. The slight sidewall roughness is caused by roughness in the gold X-ray mask absorber pattern. 


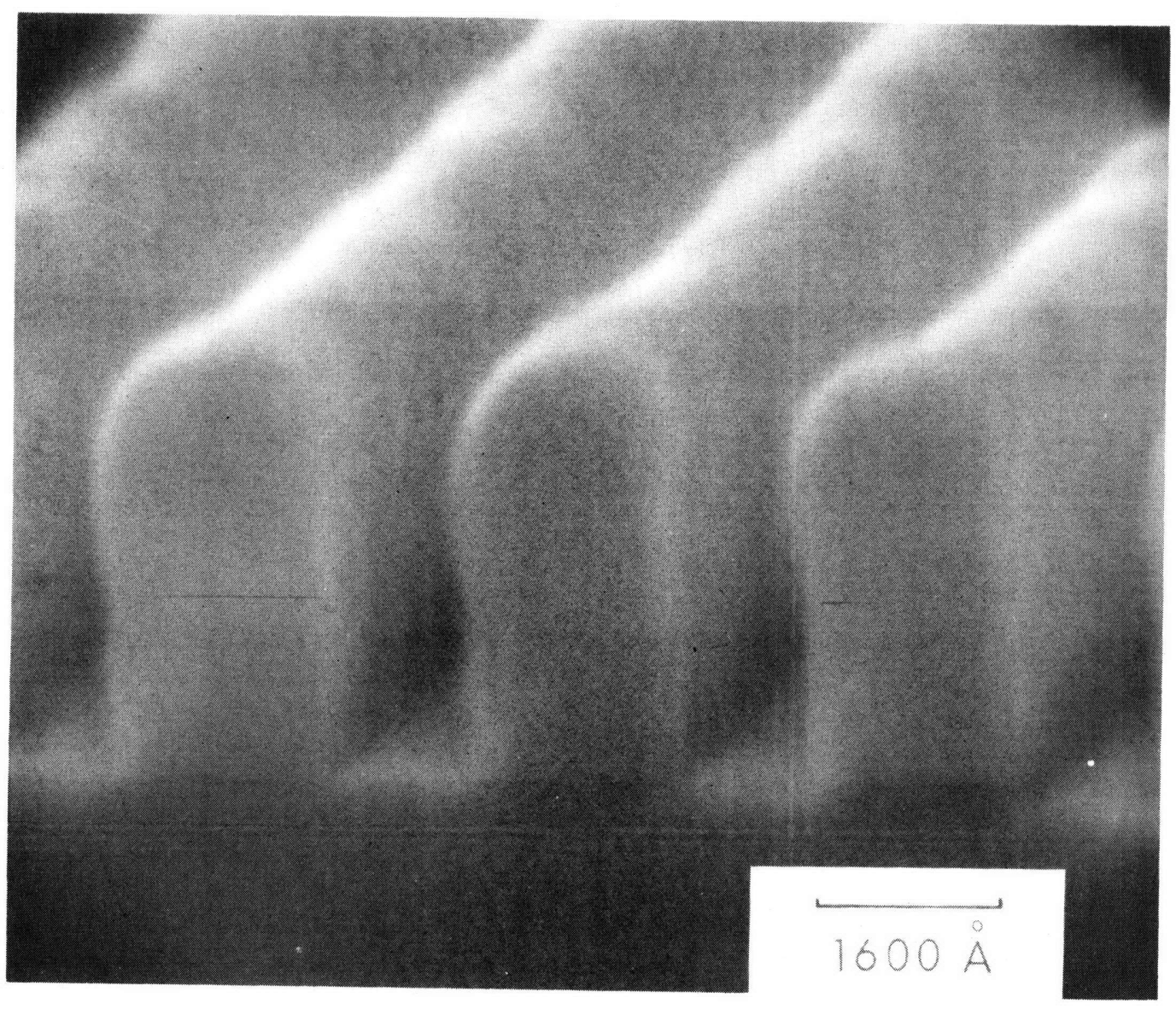

Fig. 3-17. A higher magnification SEM micrograph of the PMMA structure shown in Fig. 3-16. 


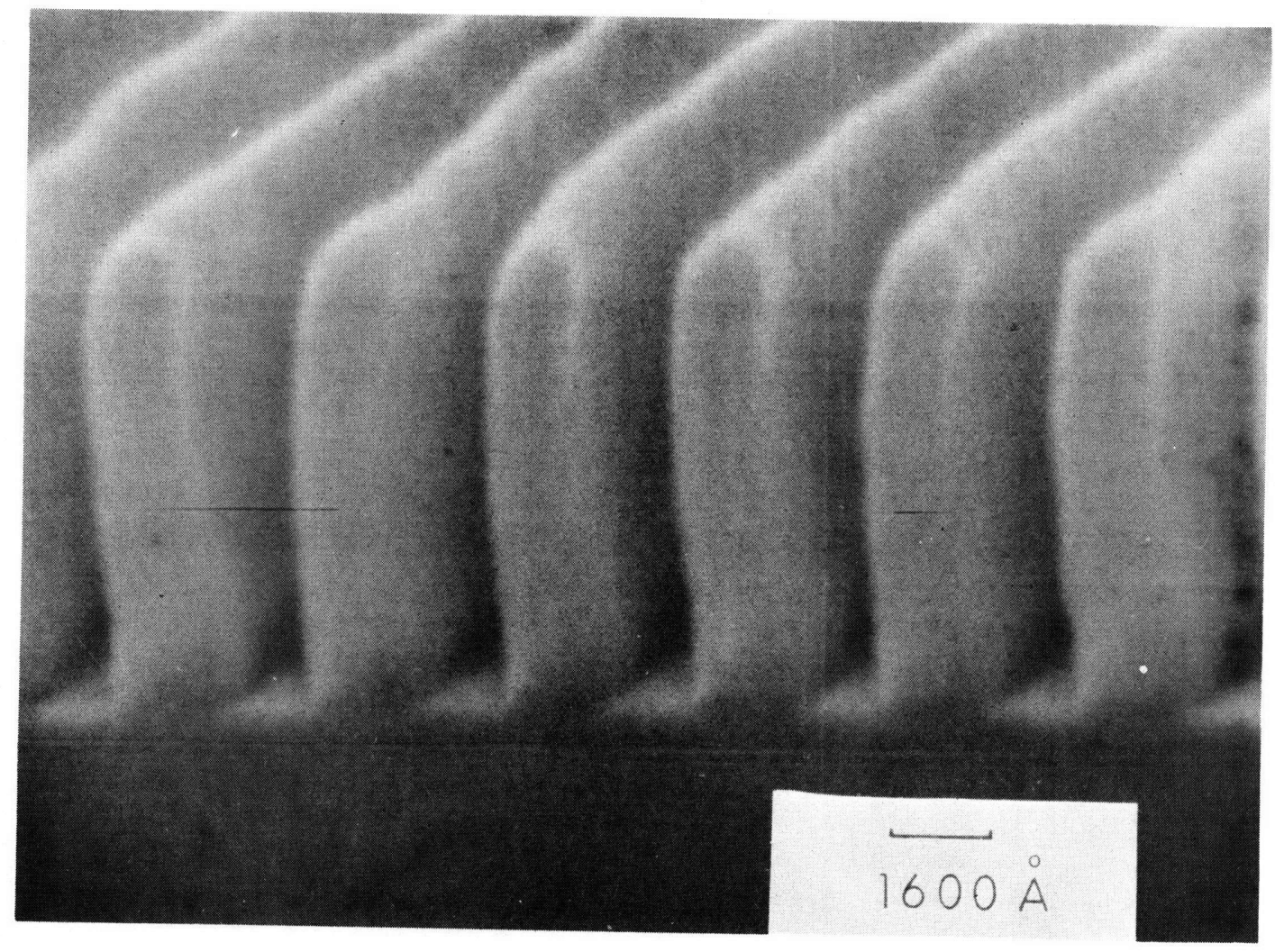

Fig. 3-18. SEM micrograph of a $320 \mathrm{~nm}$ (3200 $\AA$ ) period grating of PMMA on an amorphous $\mathrm{SiO}_{2}$ substrate exposed using the CuL $X$-ray. The grating depth is $\approx 900 \mathrm{~nm}$. The slight curvature of the PMMA slabs was probably caused by heating of the PMMA during gold coating of the sample prior to SEM examination or possibly by overdevelopment which lead to softening of the PMMA. 


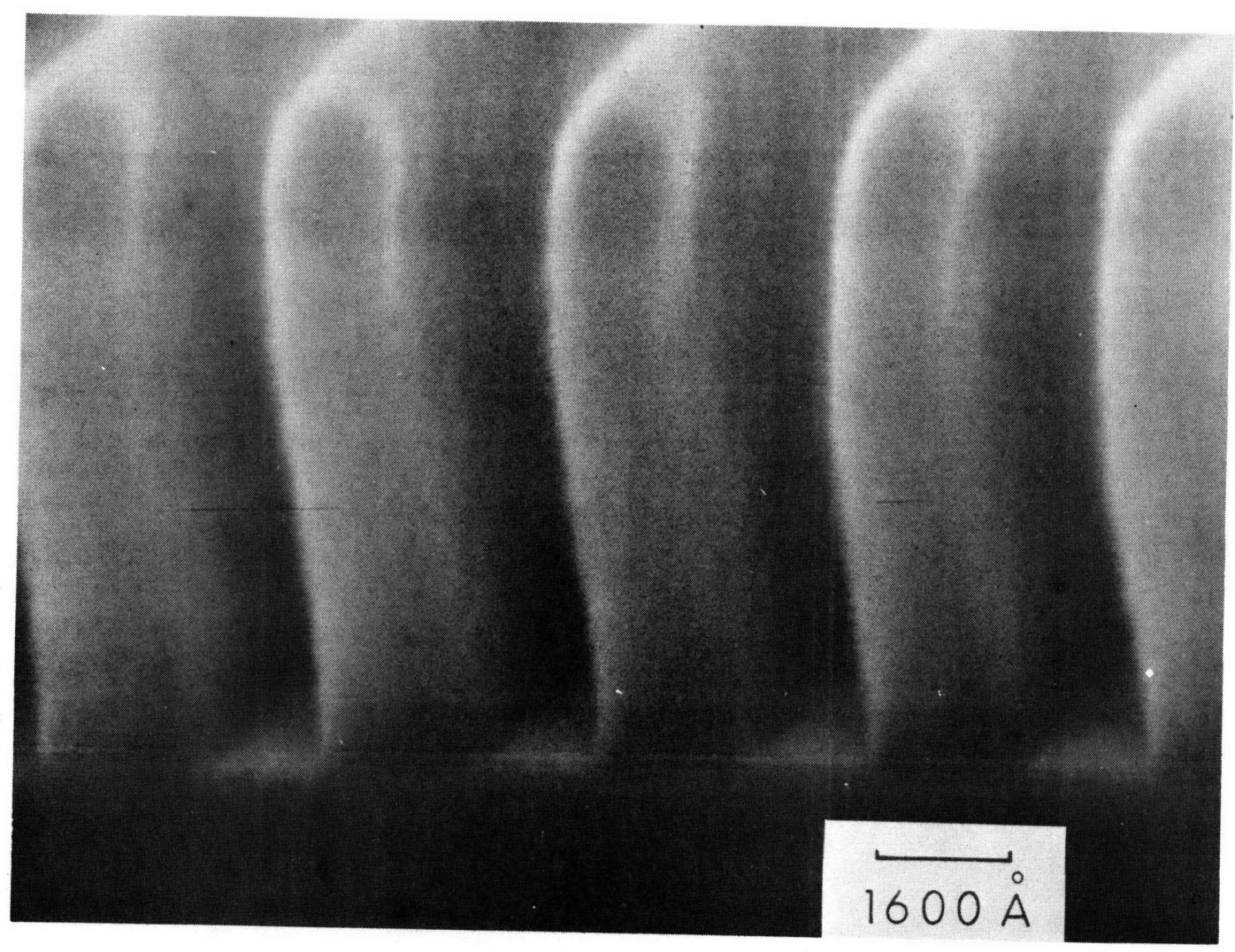

Fig, 3-19, A higher magnification SEM micrograph of the PMMA structure shown in Fig. 3-18. 


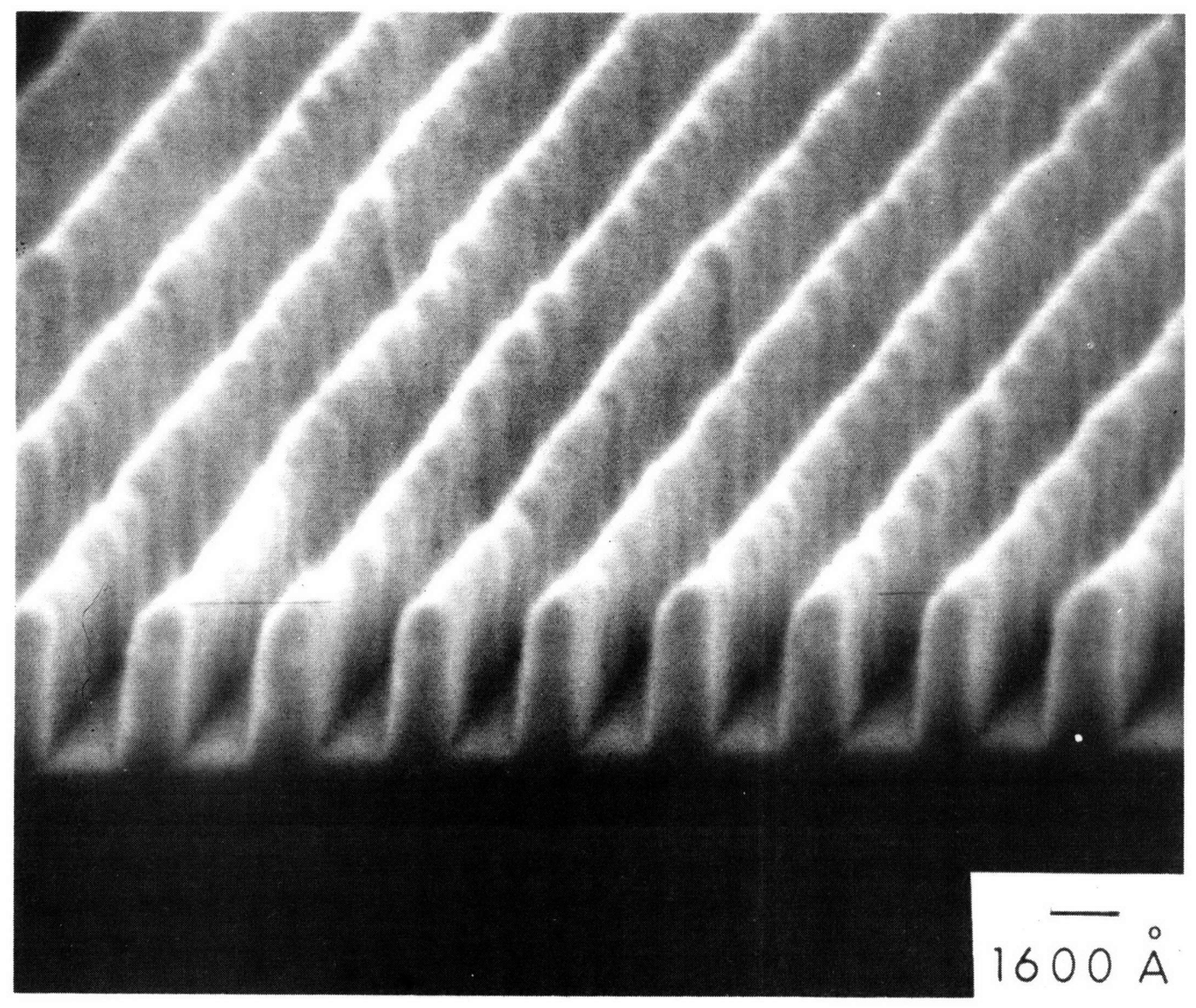

Fig. 3-20. SEM micrograph of a $320 \mathrm{~nm}(3200 \AA)$ period grating of PMMA on an amorphous $\mathrm{SiO}_{2}$ substrate exposed using the $C_{k}(44.8 \AA)$ x-ray. The sidewall roughness is believed to be a faithful reproduction of the mask edge roughness. 
power of 20 watts in 20 second intervals seperated by 5 minute cooling intervals during which the entire sputtering system was shut down. Note that the exposures done with the $\mathrm{Cu}_{L} X$-ray at $13.3 \AA$ shown in Figures 3-16 to 3-19 are smoother than the exposure done with the $C_{k} X$-ray at $44.8 \AA$. Although direct comparison is not valid because different masks were used, it appears that the mask edge roughness has been more faithfully reproduced by the carbon X-ray because of the shorter photoelectron range and higher intrinsic resolution. Also observe that the sidewalls of the $1 \mu \mathrm{m}$ deep exposure shown in Figures 3-18 and 3-19 are smoother than the shallower exposures. This is probably due to the increased penumbral blurring in the deep exposure. al though the slight curvature of the PMMA profile evident in the micrographs indicate that the structure may have been overheated during gold sputtering. Conceivably the increased smoothness could be due to surface melting. 


\subsection{ETCHING OF THE SURFACE RELIEF STRUCTURE}

To define a square-wave surface-relief structure such as illustrated in Fig. 2-2(a) a directional etching technique must be used. (Aqueous chemical etching techniques are isotropic in amorphous solids, thus they are entirely unsuitable for defining structures with vertical sidewalls.) Sputter etching ${ }^{4}$ is a highly directional etching technique which has been used extensively in the fabrication of integrated circuits, integrated optics devices, and surface acoustic wave devices. Nevertheless ion-beam sputter etching was found to be inadequate for defining straightwalled surface-relief structures because of redeposition of sputtered material ${ }^{36}$. To solve this problem in the case of $\mathrm{SiO}_{2}$ a new reactive sputter etching technique $37,38,39$ was developed in collaboration with $\mathrm{Dr}$. Hans Lehmann of RCA Laboratories in Zurich, Switzerland. The extraordinary capabilities of this technique have made $\mathrm{SiO}_{2}$ the most advantageous material for this research at the present time. 


\subsubsection{ION BEAM ETCHING-REDEPOSITION}

When the research began it was hoped that ion-beam etching would provide a general means of defining square-wave profiles. The intital results of etching of very fine linewidths were disappointing so a study of the technique was initiated. The results of this study are dramatically illustrated by the series of micrographs shown in Fig. 3-21.

The SEM micrographs show an AZ-1350J photoresist pattern on a silicon substrate prior to ion beam etching, after ion beam etching, and after removal of the photoresist by dissolution in acetone and plasma ashing in oxygen. Note that the sidewall ripple of the original photoresist profile, (a), has been replicated by the redeposited sputtered material, $(c)$. The faceting of the resist profile observed in (b) is a well known effect ${ }^{4}$ caused by the angular variation of the sputter yield.

The composition of the redeposited material is not known. It may consist entirely of redeposited substrate material or it may be some mixture of sputtered photoresist and substrate material. In any case, significant redeposition was observed with etching masks of aluminum, AZ-1350 photoresist, and PMMA after etching of such relatively low sputterrate materials as silicon, and silicon dioxide. Redeposition was not observed on high sputter-rate materials such as gold. These observations were confirmed by H.W. Lehmann 40 and P. Gloersen ${ }^{41}$. Subsequently Lehmann modeled the sputter etching process including the redeposition effect $^{40}$. Excellent agreement between the model and experiments has been obtained. 

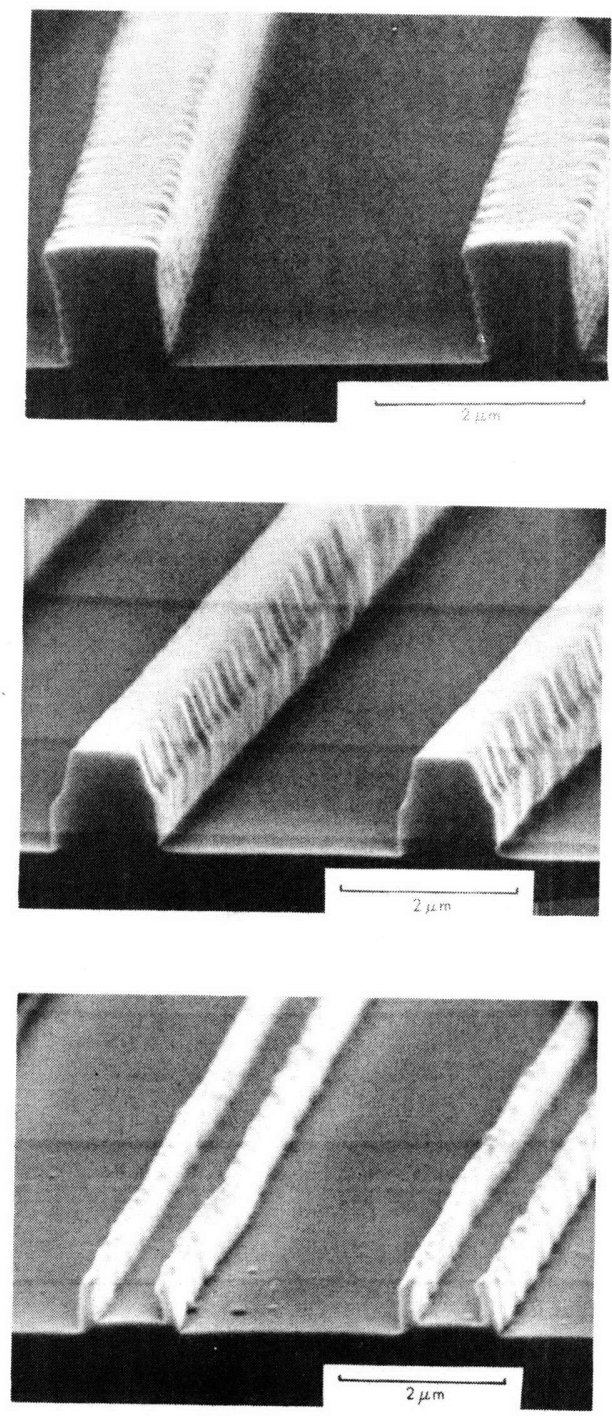

Fig. 3-21. SEM micrographs showing (Top) AZ-1350 J photoresist pattern on a silicon substrate prior to ion-beam etching, (Center) after ion-beam etching to a depth of $130 \mathrm{~nm}$, (Bottom) after dissolution of photoresist ina solvent and plasma ashing in oxygen. Note that the inner surface of the redeposited material left standing shows ripples identical to those on the sidewalls of the photoresist indicating that the redeposited material makes a cast of the photoresist. 
Lehmann's model predicts that in effect it will be impossible to obtain a square-wave vertical-walled surface-relief structure in a low sputter-rate materials such as silicon dioxide. Obviously a new technique was needed, in the case of silicon and silicon dioxide this new technique has proven to be reactive-ion etching. 


\subsubsection{REACTIVE ION ETCHING}

H. Lehmann developed a reactive-ion etching technique 37,38 for silicon dioxide for the fabrication of straight-walled-grating surface-relief structures of $1.4 \mu \mathrm{m}$ period for use in Zero Order Diffraction color filters $^{42}$. In collaboration with Dr. Lehmann the process was transferred to M.I.T. Lincoln Laboratory. The technique was then successfully applied to the etching of linewidths as small as $90 \mathrm{~nm}^{39}$. The process does not suffer redeposition problems and the etching is highly directional. Research is underway to determine the mechanism of etching which at present is not fully understood. It is believed that the highly directed ions which bombard the surface strongly enhance etching of the bombarded substrate area by the active species in the RF plasma 43 .

Etching is performed in a conventional RF sputtering system using $\mathrm{CF}_{4}$ or $\mathrm{CHF}_{3}$ gas at a pressure of $\approx 10^{-2}$ torr. The configuration is shown in Fig. 3-22. It was determined that the etch rate of $\mathrm{SiO}_{2}$ was $\approx 25 \mathrm{~nm} / \mathrm{min}$. at a total $\mathrm{RF}$ input power of 40 watts. Unfortunately the etching rate of PMMA was also found to be $\approx 25 \mathrm{~nm} / \mathrm{min}$. PMMA is therefore unsuitable as an etching mask in the reactive-ion etching process with $\mathrm{CF}_{4}$ and $\mathrm{CHF}_{3}$. To circumvent this problem a chromium mask is fabricated by the liftoff process. Chromium functions as an excellent etch mask since its etch rate is $\approx 0.9 \mathrm{~nm} / \mathrm{min}$.

The liftoff process ${ }^{4}$ used to fabricate a chromium etch-mask from a PMMA grating relief structure is shown schematically in Fig. 3-23. Chromium is evaporated (to a thickness of $10 \mathrm{~nm}$ in most cases) onto 


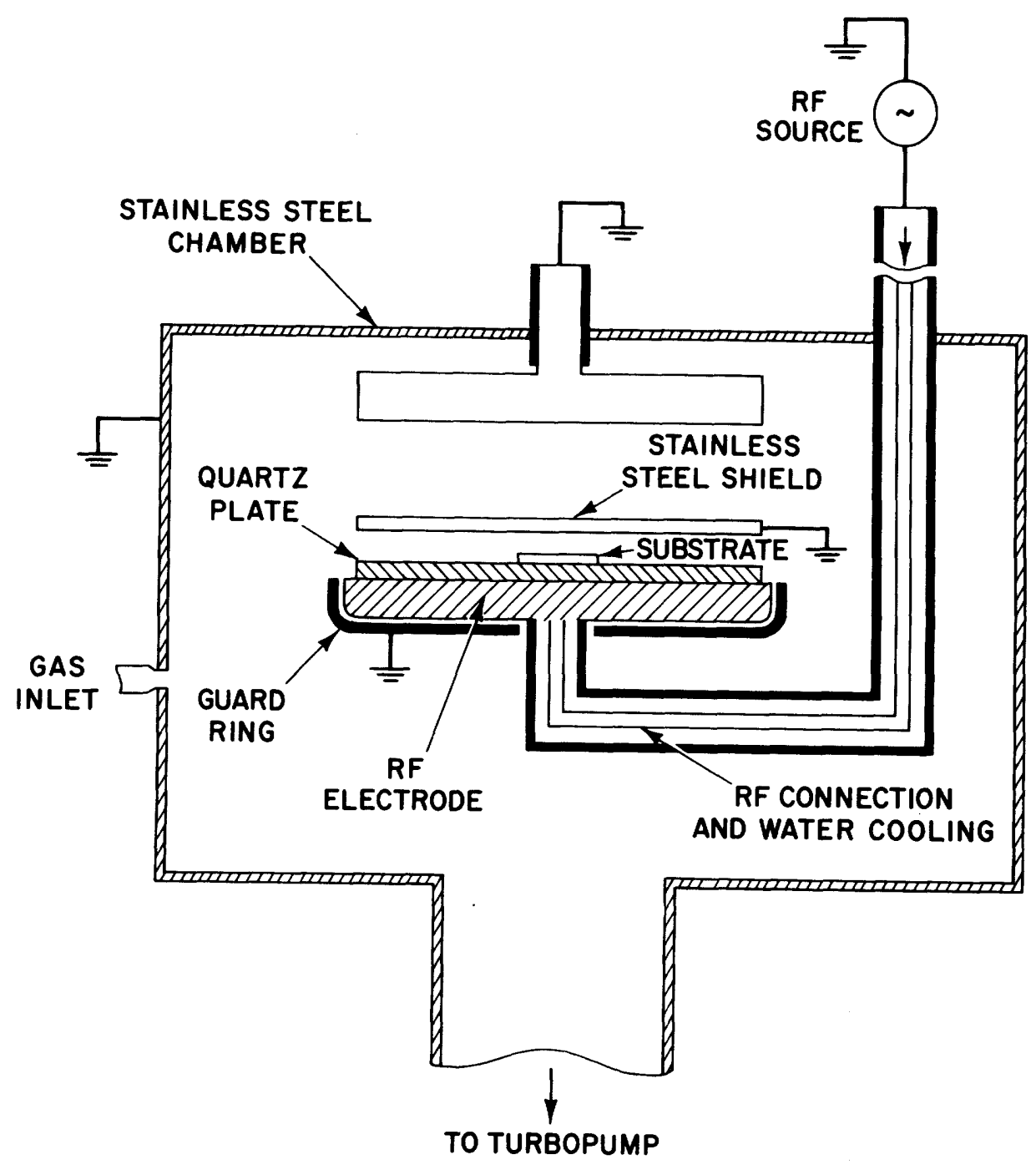

Fig. 3-22. Schematic diagram of the apparatus used to do reactive-ion etching. It is a conventional RF sputtering system where $\mathrm{CHF}_{3}$ or $\mathrm{Ci}_{4}$ gas is substituted for argon. 
18-8-14235-1]
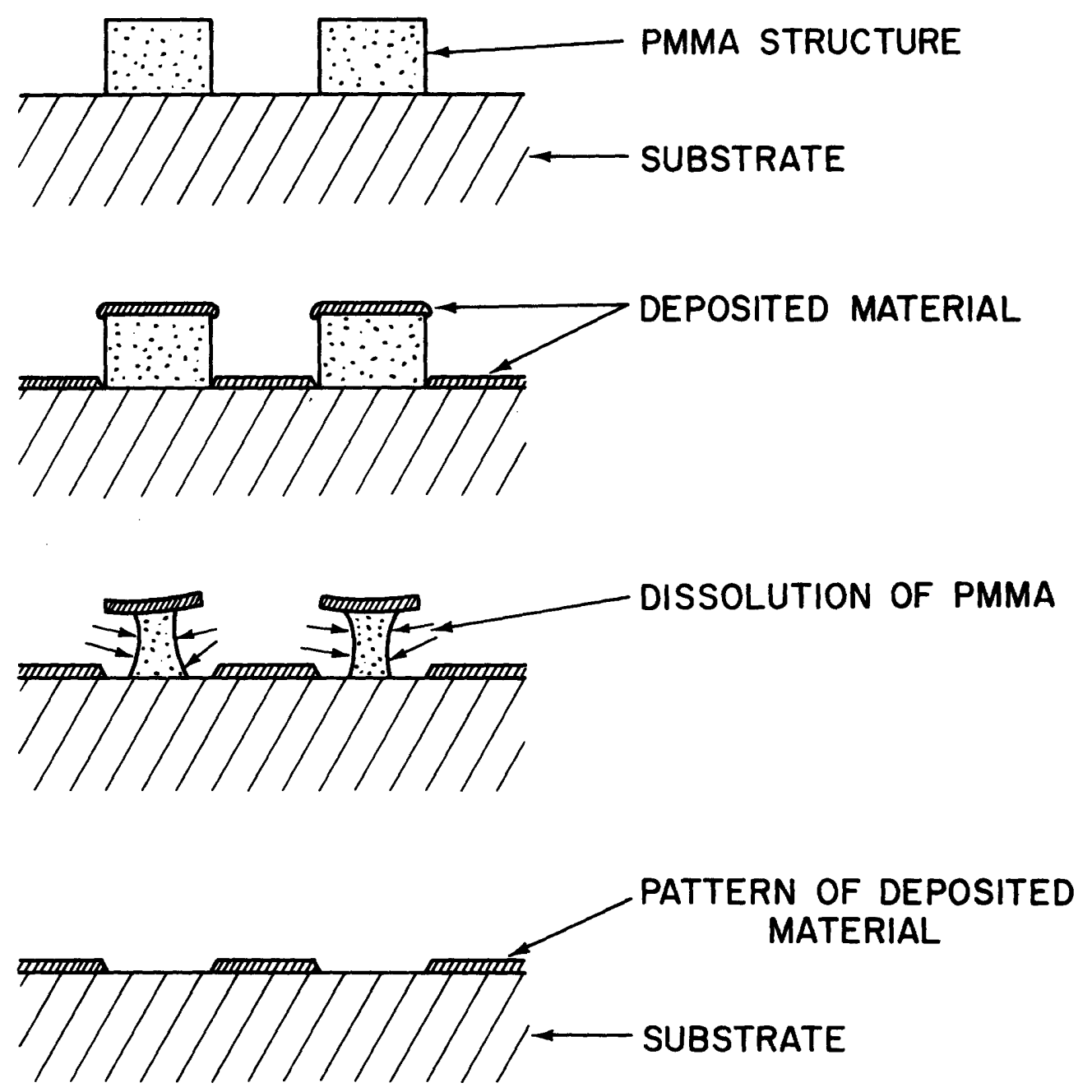

Fig. 3-23. Schematic of the sequence of steps in the liftoff process. 
the PMMA surface relief structure such that the chromium atoms arrive at normal incidence. Because the PMMA structure's walls are parallel to the incoming atoms, a continuous chrome film is not formed on the walls, The PMMA and the metal which has been evaporated upon it are subsequently removed by immersion in monochlorobenzene $\mathrm{C}_{6} \mathrm{H}_{5} \mathrm{Cl}$. (It was found that in the case of $320 \mathrm{~nm}$ period gratings ultrasonic agitation is usually necessary to completely remove the superfluous metal.)

Using a $20 \mathrm{~nm}$ thick chrome etch-mask, a $320 \mathrm{~nm}$ period surface relief structure was etched into $\mathrm{SiO}_{2}$ using $\mathrm{CHF}_{3}$ gas. The gas pressure was $1.5 \times 10^{-2}$ torr, flow rate of $15 \mathrm{cc} / \mathrm{min}$., 40 watts of R.F. power, for an etch time of 10 minutes. SEM micrographs of the resulting structure are shown in Figures 3-24 and 3-25. These structures are of course somewhat deeper than the structures employed in overlayer orientation experiments, but they do demonstrate the remarkable directionality and absence of redeposition characteristic of the reactive-ion-etching process. 


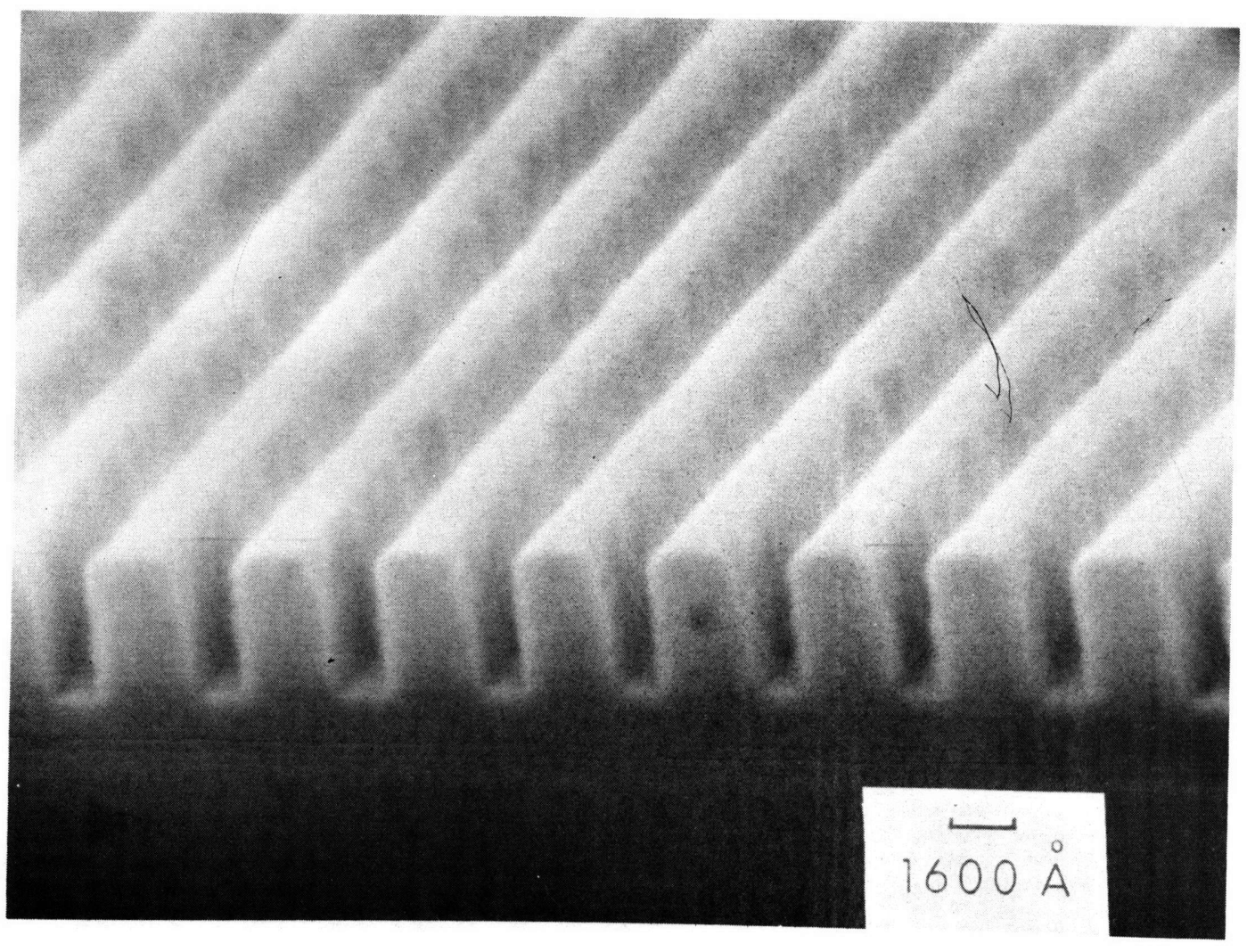

Fig. 3-24. This SEM micrograph shows a $320 \mathrm{~nm}$ ( $3200 \AA$ ) period surface relief structure in amorphous $\mathrm{SiO}_{2}$. The structure was fabricated by reactive-ion etching with $\mathrm{CHF}_{3}$ gas through a $20 \mathrm{~nm}$ thick chromium mask. The chromium mask was fabricated on the substrate by liftoff using the PMMA structure shown in Fig. 3-16. 


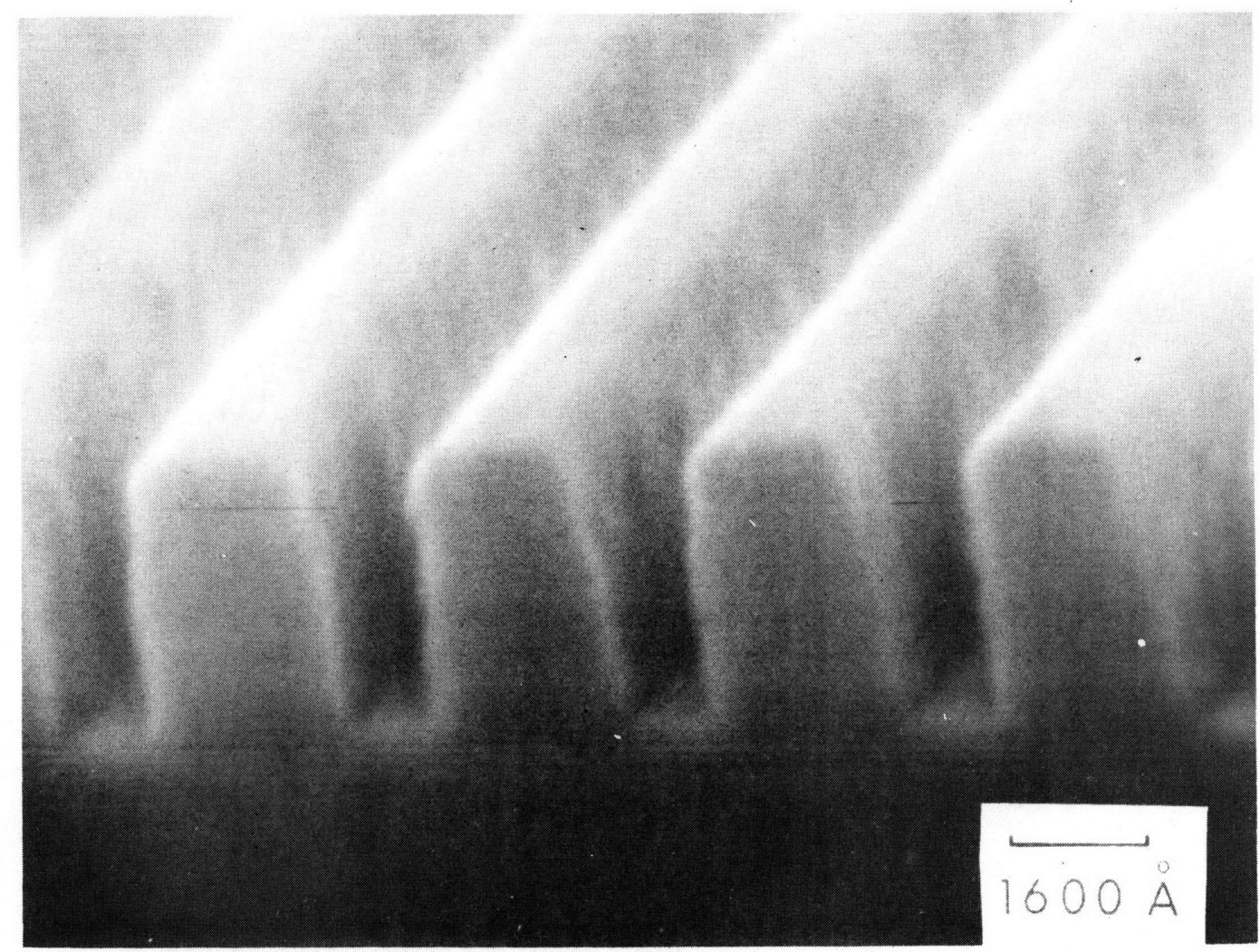

Fig. 3-25. Higher magnification SEM micrograph of the amorphous $\mathrm{SiO}_{2}$ surface relief structure shown in Fig. 3-24. 


\subsubsection{FINAL CLEANING}

Following reactive-ion etching, the chromium etching mask is chemically removed using an aqueous chromium etch (Ceric Ammonium Nitrate, perchloric acid, and water, $164.5 \mathrm{grms}, 43 \mathrm{ml}$, and $1000 \mathrm{ml}$ respectively) ${ }^{*}$ and the substrate is given a thorough rinse in water. At this point it has been observed that the substrates do not wet uniformly in water indicating some surface contamination. Researchers have found that a thin layer of carbon can be deposited on a substrate during reactive-ion etching with $\mathrm{CHF}_{3}{ }^{43}$. To remove any carbon or organic contamination the substrates were subjected to UV-Ozone cleaning ${ }^{44}$. The samples were placed approximately $2 \mathrm{~mm}$ from a quartz low pressure mercury $1 \mathrm{amp}^{45}$ in atmosphere for 30 minutes. The combined action of ozone and ultraviolet light is known to be effective in the removal of carbon and organic residues. Following this treatment substrates were found to wet uniformly indicating a clean uncontaminated $\mathrm{SiO}_{2}$ surface.

* This is sometimes designated Kodak Chrome Etch. 


\subsection{CHARACTERIZATION OF SURFACE RELIEF STRUCTURES}

The ability to observe and characterize surface relief structures before and after fabrication is of great importance. Without precise knowledge of the results of a procedure process development and improvement are very difficult.

For observations where resolution of only $10 \mathrm{~nm}$ is required Scanning Electron Microscopy (SEM) is adequate. A drawback of the SEM is the necessity of coating nonconducting samples with metal which can alter or obscure detail. Another difficulty is that electron diffraction cannot be done in the SEM to determine the crystallinity of a sample.

To obtain higher resolution, perform selected area diffractions, and avoid the necessity of coating samples conventional Transmission Electron Microscopy (TEM) or Scanning Transmission Electron Microscopy (STEM) must be used. The price one pays for these improvements over SEM is that the samples must be very thin $\left(<200 \mathrm{~nm}\right.$ thick for $\left.\mathrm{SiO}_{2}\right)$ and of small diameter $(<3 \mathrm{~mm})$.

Sample thinning prior to observation by TEM or STEM is done using the techniques developed for the fabrication of $\mathrm{Si}_{3} \mathrm{~N}_{4}-\mathrm{SiO}_{2} \mathrm{X}$-ray masks. As described in Appendix A, a film of $\mathrm{Si}_{3} \mathrm{~N}_{4}$ approximately $100 \mathrm{~nm}$ thick and $\mathrm{SiO}_{2}$ approximately $100 \mathrm{~nm}$ thick is grown on a $\{100\}$ silicon wafer, or a film of approximately $200 \mathrm{~nm}$ of $\mathrm{SiO}_{2}$ alone is grown. A surface.relief structure is then fabricated in the $\mathrm{SiO}_{2} \mathrm{film}_{\text {. When }}$ structure is completed or after an overlayer deposition experiment, the underlying silicon is etched away. The silicon substrate is placed in 
a special holder (see Appendix A) which protects the surface relief structure. The silicon is anisotropically etched through a mask pattern on the back of the silicon using a solution of Ethylene Diamine Pyrocatachol in water. ${ }^{83}$ The mask pattern on the back of the silicon defines small square frames which support the thin $\approx 200 \mathrm{~nm}$ thick membrane.

After thinning the samples can be viewed directly by either TEM or STEM. TEM micrographs of an $\mathrm{SiO}_{2}$ structure similar to that shown in the SEM micrographs of Figures 3-24 and 3-25 are shown in Fig. 3-26. Careful measurement of these and other TEM micrographs indicate that the sidewalls of square-wave surface-relief structures fabricated using reactive-ion etching deviate a maximum of 6 degrees from vertical and the radii of curvature of the top edge and groove corners are less than $5 \mathrm{~nm}$. 

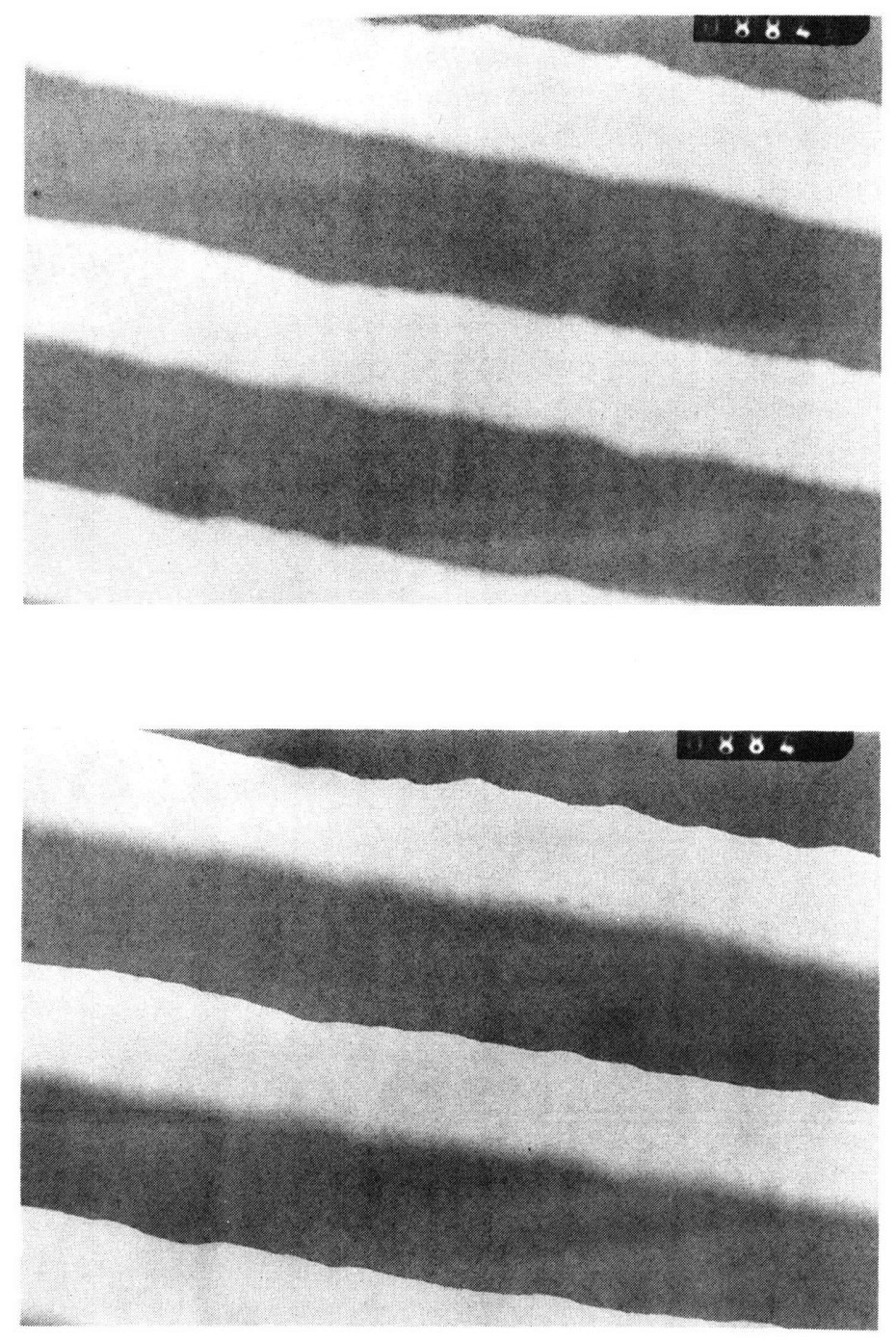

Fig. 3-26. TEM micrographs are shown of a $320 \mathrm{~nm}$-period $50 \mathrm{~nm}$-deep square-wave surface-relief structure in amorphous $\mathrm{SiO}_{2}$. The upper micrograph was taken with the sample tilted $0^{\circ}$ with respect to the electron beam. The lower micrograph was taken with the sample tilted $12^{\circ}$ with respect to the electron beam. The magnification is $150 \mathrm{kx}$. 


\subsection{CONCLUSION}

New $X$-ray lithographic and reactive-ion etching techniques have been developed. These techniques have been applied to the fabrication of $160 \mathrm{~nm}$ linewidth square-wave gratings in $\mathrm{SiO}_{2}$ for use in overlayer orientation experiments. Improvements in the quality of the gold absorber pattern of $X$-ray masks, improved $1 \mathrm{iftoff}$ techniques, and a better understanding of reactive-ion etching should lead to further improvement in the smoothness of the sidewalls and sharpness of the edges of square-wave gratings. The techniques themselves are quite general and can be used for the fabrication of a wide variety of structures with submicrometer dimensions. 


\section{ORIENTATION OF LIQUID CRYSTALS BY ARTIFICIAL \\ SURFACE RELIEF STRUCTURES}

\subsection{INTRODUCTION}

As explained in section 2.5, liquid crystals are a particularly convenient material to use to test the concept of orientation of anisotropic overlayer materials by surface relief structures. The interfacial tensions of liquid crystals are highly anisotropic, their orientation is easily measured by optical techniques, and an equilibrium configuration is easily achieved because of the high mobility of the molecules in the 1iquid crystal. In this Chapter, the properties of the nematic and smectic A phases of liquid crystals are discussed, a proposed mechanism of liquid crystal orientation by an artificial surface-relief structure is presented, and details are given of an experimental demonstration of uniform "single crystal" orientation of nematic and smectic A liquid crystals by square-wave surface-relief gratings in amorphous $\mathrm{SiO}_{2}$. 


\subsection{PROPERTIES OF LIQUID CRYSTALS}

Certain phases of some organic materials whose molecules are elongated have mechanical properties and symmetry properties which are intermediate between those of a liquid and those of a solid crystal ${ }^{5}$. For this reason such materials are often called liquid crystals. The more proper name is mesomorphic phases (mesomorphic meaning; of intermediate form). The molecules of a conventional isotropic liquid are both positionally and orientationally disordered while in a crystal the molecules are located and oriented on a three dimensional lattice. Two of the possible mesophases which are of interest here are:

(i) Nematic phases, where certain organic liquids show a low temperature phase where the elongated molecules are aligned preferentially along one direction. They are positionally disordered, but orientationally ordered. At higher temperatures they undergo a transition to a conventional isotropic liquid phase.

(ii) Smectic phases, obtained by imposing positional order in one direction only. Such a system is simply a set of two dimensional liquid layers stacked on each other with a well defined spacing. If the molecules are oriented normal to the layers, within each layer, it is known as a smectic A phase.

Fig. 4-1 illustrates schematically the nematic and smectic $A$ phases. Some liquid crystal materials exhibit a series of phase transitions from isotropic, to nematic, to smectic, to crystalline as the temperature is lowered. The sequence is clear from Fig. 4-1. 

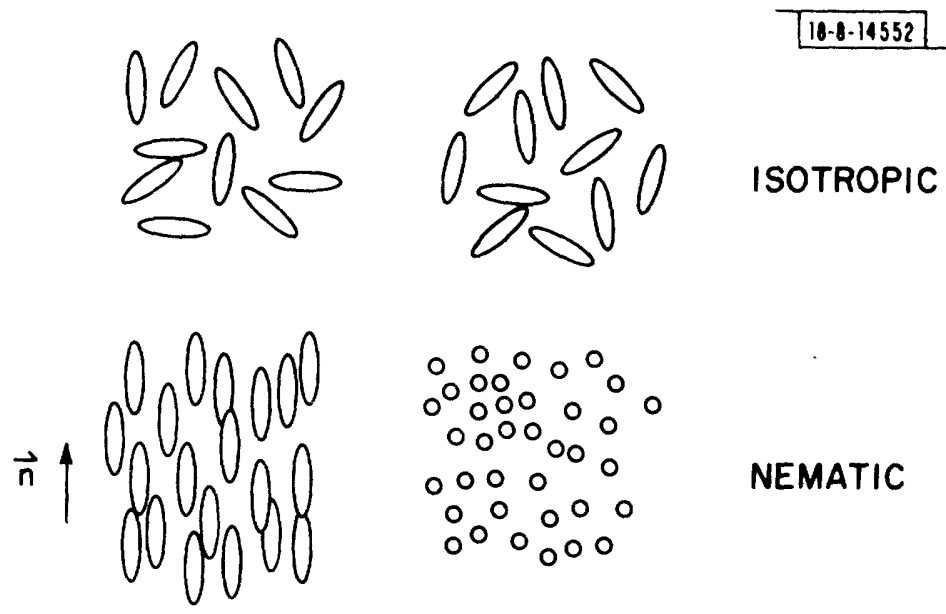

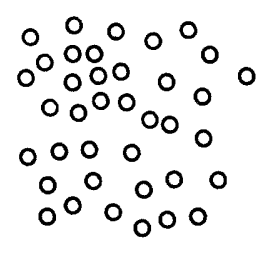

NEMATIC

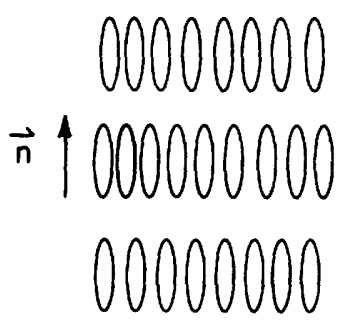

$$
\begin{aligned}
& \text { ०० } 0000 \\
& \text { } \\
& 0000000
\end{aligned}
$$

SMECTIC A

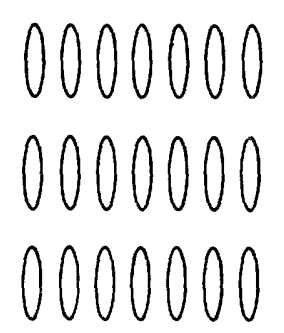

0000000 0000000 0000000 0000000 0000000 0000000

CRYSTAL 0000000

Fig. 4-1. Schematic representations of the isotropic, nematic, smectic $A$ and crystalline phases of a liquid crystal material. 
The local orientation of both a nematic and smectic $A$ phase can be specified by a unit vector, $\vec{n}$, which is parallel to the average direction of the elongated molecules. Since both the smectic A and nematic phases are optically uniaxial the local optic axis will also correspond to $\vec{n}$. The terms optic axis and director are thus interchangeable.

The polar plot of the interfacial tension, $\gamma(\vec{n})$, of an interface between a nematic or smectic A phase and a nonordered phase will be a smooth nonspherical surface as described in section 2.2 Since the function $\gamma(\vec{n})$ is cylindrically symmetric the entire plot can be represented as a two dimensional curve. The interfacial tension of interest here will be that of the specific liquid crystals MBBA and M-24. (See Appendix D for properties of these materials.) The entire plot of $\gamma(\vec{n})$ is not available for either material. However, the orientation at which the minimum interfacial tension occurs can be determined by observing the orientation of a thin slab of the liquid crystal confined between two identical interfaces. 5,46 The equilibrium orientation will correspond to the orientation at which the minimum interfacial tension occurs. The possible results of such a measurement are shown in Fig. 4-2.

For smooth clean glass or $\mathrm{SiO}_{2}$ surfaces, most investigators 5,47 report that the minimum interfacial tension occurs with, $\vec{n}$, parallel to the surface (Fig. 4-2(C)) for MBBA and M-24. By coating an $\mathrm{SiO}_{2}$ surface with various silane compounds ${ }^{48}$ or lecithin ${ }^{49}$ it is possible to obtain a surface with the minimum interfacial tension at the orientation where $\vec{n}$ is perpendicular to the surface (Fig. 4-2(a)). Other surface treatments 


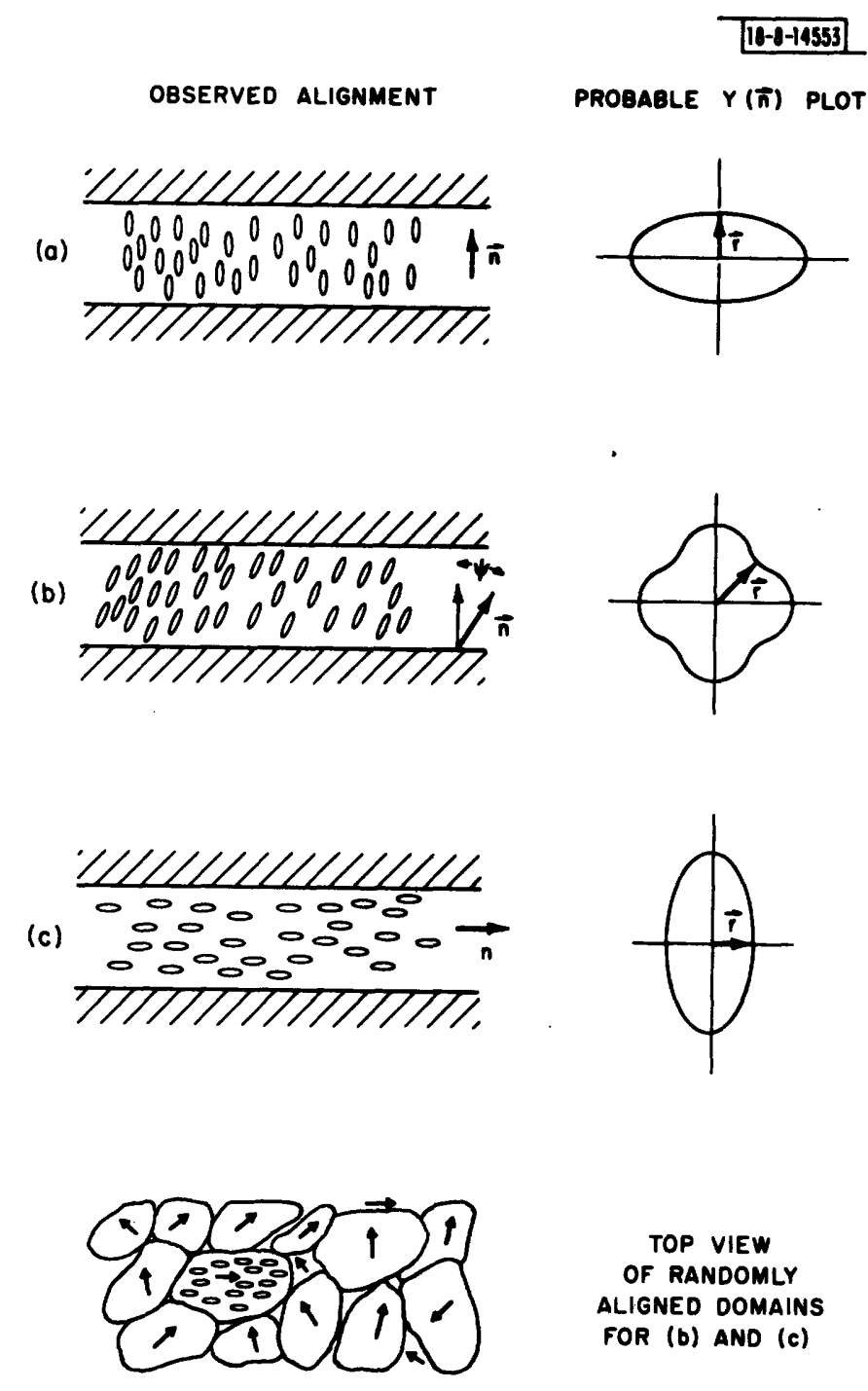

Fig. 4-2. The possible equilibrium orientations of a nematic (or smectic) liquid crystal confined between smooth parallel interfaces. In practice the component of $\vec{n}$ in the plane of the interfaces will have no preferred direction for a smooth amorphous material. In this case the confined liquid crystal will be composed of small randomly oriented domains as shown in the lower diagram. 
including certain silanes 48 and evaporated carbon ${ }^{50}$ can also produce surfaces with minimum interfacial tension for, $\vec{n}$, parallel to the surface. It is worth nothing that Creagh and Kmetz have claimed that MBBA will orient with, $\vec{n}$, perpendicular to a glass surface if it is "thoroughly cleaned" with chromic acid. It appears likely that the glass surface has been etched by their "cleaning" step and the surface is no longer planar ${ }^{5}$. In any event, it is well established that the minimum interfacial tension of MBBA and M-24 occurs for $\vec{n}$ parallel or very near parallel to a conventionally cleaned smooth $\mathrm{SiO}_{2}$ surface.

For many experimental purposes 5 and for some commercially important nematic liquid crystal display devices 46 it is often desirable to maintain uniform alignment of the director, $\vec{n}$, parallel to a substrate surface in some specified direction. In practice, uniform parallel alignment is not obtained between parallel smooth amorphous substrate surfaces even though the minimum interfacial energy occurs for $\vec{n}$ parallel to the substrate. This is explained by noting that the interfacial tension is isotropic for variations of the director in the substrate plane, thus the equilibrium orientation in the plane is indeterminate, and very small random perturbations can cause the orientation of the director to wander in the plane of the substrate over a macroscopic sample. Samples of nematic liquid crystal confined between smooth glass or amorphous $\mathrm{SiO}_{2}$ surfaces where the interfacial tension is a minimum for $\vec{n}$ parallel to the substrate tend to be made up of regions where $\vec{n}$ varies slowly (on the order of a micrometer in diameter) separated by disclinations ${ }^{5,51}$ (see Fig. 4-2). Smectic A 1iquid 
crystals confined in the same manner also tend to form domains or grains of micrometer size separated in this case by both dislocations and disclinations 5,51 .

It has been determined empirically that uniform parallel alignment of nematics and smectics can be induced by employing surfaces which are anisotropic in the plane. Successful orientation has been obtained on planar single crystal surfaces ${ }^{52}$ (i.e., heteroepitaxy of liquid crystals) and on surfaces which have been unidirectionally rubbed with paper, cotton, rouge, leather, diamond paste, etc. ${ }^{5,46}$. Alignment has also been obtained on $\mathrm{SiO}_{x}$ surfaces evaporated at a steep angle 53,54 and on surfaces which have been unidirectionally pulled from various solutions ${ }^{55}$. In all cases the liquid crystal alignment direction coincides with the direction of rubbing, pulling, evaporation, crystal anisotropy etc.

Various models $56,48,57,58$ have been proposed to explain the observed alignment in the case of rubbing and evaporation of $\mathrm{SiO}_{2}$. The most generally accepted model is Berreman's ${ }^{56}$ which maintains that surface relief structures produced on the substrate by the rubbing and evaporation processes lead to an anisotropy in the interfacial tension for variations of the director in the plane such the the minimum total free energy configuration occurs with the director parallel to the rubbing or evaporation direction. Direct correlation of surface structure with orientation has been difficult because of uncertainties in the preparation of the surfaces and the difficulty of observing the surface 57 . Now that well controlled surface relief structures on amorphous substrates can be 
prepared using the techniques described in Chapter III, a direct test of the theory of liquid crystal orientation by surface relief structures is possible. 


\subsection{THE GENERALIZED INTERFACIAL TENSION OF A LIQUID CRYSTAL AND A SURFACE RELIEF STRUCTURE}

If the interface between a liquid crystal and a substrate is not planar but is described by the periodic function, I $(x, y)$, the generalized interfacial tension $\sigma_{\ell c}(\vec{n})$ as defined in section 2.3 will not be isotropic in the substrate plane. In general the interfacial tension $\sigma_{l c}(\vec{n})$ will now be described by a polar plot which will be a surface which is not cylindrically symmetric.

Insight into the form of $\sigma_{\ell c}(\vec{n})$ for an interface described by $I(x, y)$ can be gained by considering the interfaces illustrated in Fig. 4-3. Note that the liquid crystal can be distorted in the interface region as in Figs. 4-3(a) and 4-3(b). Associated with this distortion is a distortion free energy, $F_{d}$. In general the total interfacial tension will consist of a "physiochemical part", determined by the interfacial tension of the liquid crystal at the liquid crystal-substrate discontinuity, and a distortion free energy part determined by the excess free energy resulting from the surface relief structure induced distortion. The total interfacial tension will thus be given by

$$
\sigma_{l c}(\vec{n})=\int_{I(x, y)}^{\gamma_{l c}}\left(\vec{n}_{i}(x, y, z)\right) d A+\int F_{\text {Interface region }} F_{d}(x, y, z) d v
$$

Where $\vec{n}$ specifies the orientation just outside the interface region, and $\vec{n}_{j}(x, y, z)$ gives the local director.

The distortion free energy for a nematic liquid crystal can be 
(a)

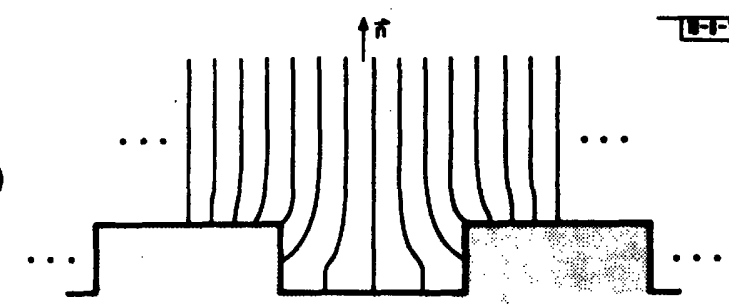

(b)

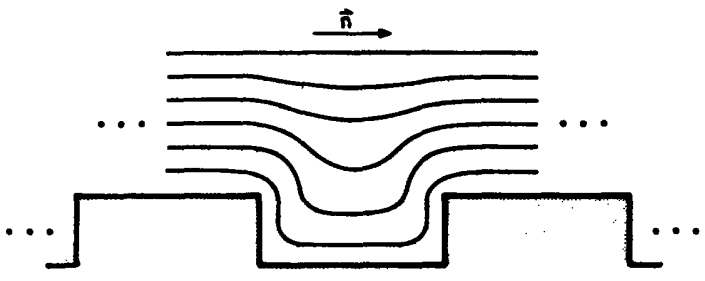

(c)

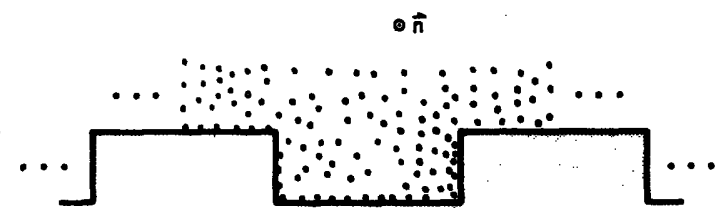

(d)

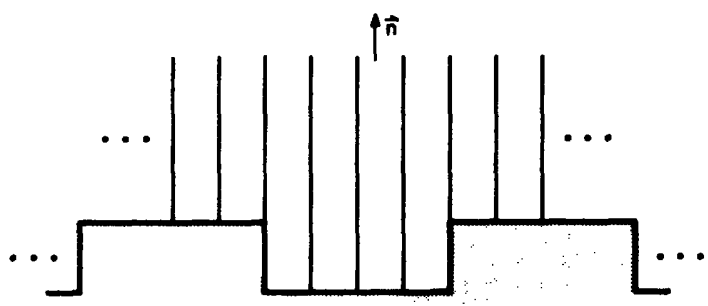

Fig. 4-3. Schematic illustration of possible configurations of a nematic liquid crystal at a square-wave grating interface. The director is tangent to the curves drawn above the interfaces. a). Configuration for $\vec{n}$ perpendicular at $z=I(x, y)$. b). Configuration for $\vec{n}$ tangent to $z=I(x, y)$ and perpendicular to the $y$ or groove direction. c). Configuration for $\vec{n}$ tangent to $z=I(x, y)$ and parallel to the groove direction. d). Configuration where $\vec{n}$ is everywhere perpendicular to the $x-y$ plane. 
written in general as

$$
\begin{aligned}
& F_{d n}=1 / 2 K(\operatorname{div} \vec{n})^{2}+1 / 2 K_{2}(\vec{n} . \operatorname{curl} \vec{n})^{2} \\
& +1 / 2 K_{3}(\vec{n} \times \operatorname{curl} \vec{n})^{2}
\end{aligned}
$$

Where $K_{1}, K_{2}$, and $K_{3}$ are the splay, twist, and bend elastic coefficients. Assuming that the interplanar spacing remains constant, the distortion free energy for a smectic $A$ phase is given by

$$
F_{d s}=1 / 2 K_{1}(\operatorname{div} \vec{n})^{2}
$$

Both the bend and twist distortion are forbidden in the smectic $A$ so these terms are absent.

Evidently the minimum interfacial tension $\sigma_{\ell c}(\vec{n})$ will be obtained if the director orientation is constant in the interface region making $F_{d}=0$ and $\int r_{\ell c} n_{i}(x, y, z) d A$ is a minimum. This will be the case for both nematics $I(x, y)$ and smectics $A$ on a square-wave grating such as shown in Fig. 4-3(c), when $\gamma_{\ell c}(\vec{n})$ is a minimum for $\vec{n}$ parallel to a smooth surface of the grating material. If the minimum of $\gamma_{\ell c}(\vec{n})$ should occur at some orientation other than parallel, then the orientation at which minimum $\sigma_{\ell c}(\vec{n})$ occurs will be determined by the competition between the distortion free energy term and the physiochemical interfacial tension term. This will also be the case if the surface relief structure varies in both $X$ and $Y$ directions since there will be no orientation where the distortion free energy term vanishes. 


\subsection{THE ORIENTATION OF LIQUID CRYSTALS BY SQUARE WAVE GRATINGS ON AMORPHOUS $\mathrm{SiO}_{2}$ SUBSTRATES - EXPERIMENT}

Several liquid crystal alignment experiments were done in the following manner. Square-wave grating structures were fabricated on amorphous $\mathrm{SiO}_{2}$ substrates using the techniques described in Chapter III. A "sandwich" consisting of two substrates with surface-relief grating structures oriented parallel to one another, spacers, and liquid crystal was then assembled as shown in Fig. 4-4. The orientation of the liquid crystal layer was measured by observing the sandwich in transmitted light between crossed polarizers in an optical microscope. Uniform alignment was observed (as predicted) in the direction of the grating grooves for $M-24$ in both the nematic and smectic A phases. The same result was obtained for the nematic phase of MBBA. 

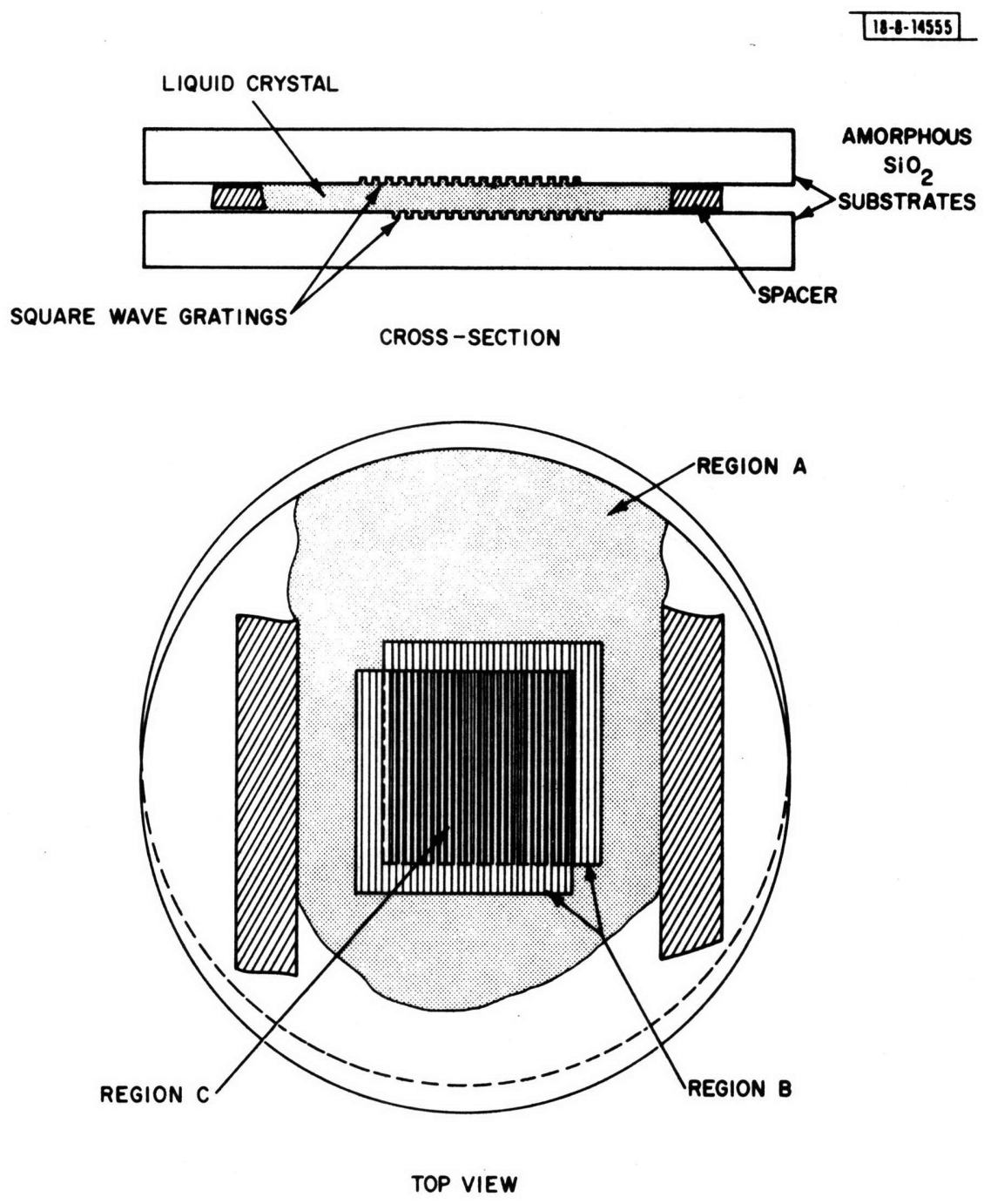

Fig. 4-4. A schematic cross-section and top view are shown of the "sandwich" assembly used to investigate surface-relief structure orientation effects in nematic and smectic A liquid crystals. 


\subsubsection{THE GRATING STRUCTURES}

Two substrates were prepared with grating surface-relief structures

using identical procedures. Optically polished fused quartz substrates 59 (amorphous $\mathrm{SiO}_{2}$ ) $3.75 \mathrm{~cm}$ in diameter, and $1.5 \mathrm{~mm}$ thick were used.

A $320 \mathrm{~nm}$ period grating, $1.25 \mathrm{~cm}$ square, was exposed in $300 \mathrm{~nm}$ of PMMA using $\mathrm{Cu}_{L} X$-ray lithography. Following development of the $X$-ray exposure $10 \mathrm{~nm}$ of chromium was lifted off. The substrate was then reactive-ion etched in $\mathrm{CHF}_{3}$ gas to a depth of $25 \mathrm{~nm}$. Finally the chromium was chemically removed using an aqueous cheomium etch. Prior to the first alignment experiments the only cleaning of the completed substrate relief structure was a thorough rinse in distilled water. At that point the surface was not wet by water indicating the presence of surface contamination. The most likely contaminent appears to have been a carbon film produced during reactive-ion etching ${ }^{43}$. After the first alignment experiments the substrates were cleaned with organic solvents and were subjected to UV ozone cleaning ${ }^{44}$. This cleaning procedure resulted in uniform wetting of the substrates by water indicating an uncontaminated $\mathrm{SiO}_{2}$ surface. However, no significant difference was noticed between alignment experiments using substrates which wet and those that did not. 


\subsubsection{ALIGNMENT OF MBBA IN THE NEMATIC PHASE}

Two substrates were assembled in a sandwich as shown in Fig. 4-4 using two 50 micrometer thick teflon spacers. To align the two gratings parallel to one another the light diffracted by the two gratings from an incident collimated light beam was observed while rotating the samples relative to one another. Near grating groove parallelism a moire pattern was observed. By minimizing the number of fringes in the moire pattern the two gratings were aligned parallel to within less than one milliradian. 60 The grating areas on the two substrates did not overlap exactly, because of this, the area between the two substrates was comprised of three distinct regions:

A. A region outside the grating area of both substrates where smooth surfaces faced each other.

B. Regions where a grating on either the top or bottom substrate faces a smooth substrate area.

C. A region where parallel gratings face one another.

Following assembly the entire sandwich was heated on a hotplate to a temperature above the nematic-isotropic transition of MBBA. MBBA was then introduced between the substrates by capillary action. The sandwich was removed from the hot plate and allowed to cool rapidly to room temperature. Upon transition to the nematic phase at $\approx 46.5{ }^{\circ} \mathrm{C}$ a large number of defects or disclinations ${ }^{51}$ were observed in all regions of the sandwich. The density of defects began to decrease immediately. After about 60 seconds the density of disclinations in region $C$ was much lower than in region $A$. 
The density of defects in region $B$ was intermediate.

The sandwich was observed in transmission in a microscope between crossed polarizers. The incident light arrived normal to the thin liquid crystal layer and the entire sandwich could be rotated in the plane of the layer.

An aligned nematic or smectic A liquid crystal behaves as an optically uniaxial medium with its optic axis parallel to the director $\vec{n}$. From the indicatrix construction ${ }^{61}$ one sees that linearly polarized light passing through a slab of uniaxial medium at normal incidence will remain linearly polarized (for all frequencies and slab thicknesses) only if the direction of propagation, $\vec{k}$, the polarization direction $\vec{D}$, and the optic axis, $\vec{n}$, lie in the same plane, or if $\vec{D}$ is in the direction of $\vec{K} \times \vec{n}$ In other words, a normally incident linearly polarized wave will remain linearly polarized if the incident light is polarized parallel or perpendicular to the projection of the director on the plane of the slab. Thus, by observing the light transmitted through a uniaxial slab between crossed polarizers one can determine the direction of the in plane component of the director to within 90 degrees by finding the angular orientation of the slab relative to the polarizers which results in a transmission null.

The intensity of the light passing through the MBBA liquid crystal sandwich between crossed polarizers was observed in regions $A, B$, and $C$, as the sandwich was rotated relative to the polarizers. Within region A a complex pattern of disclinations was observed. A micrograph of the type of pattern observed is shown in Fig. 4-5. 


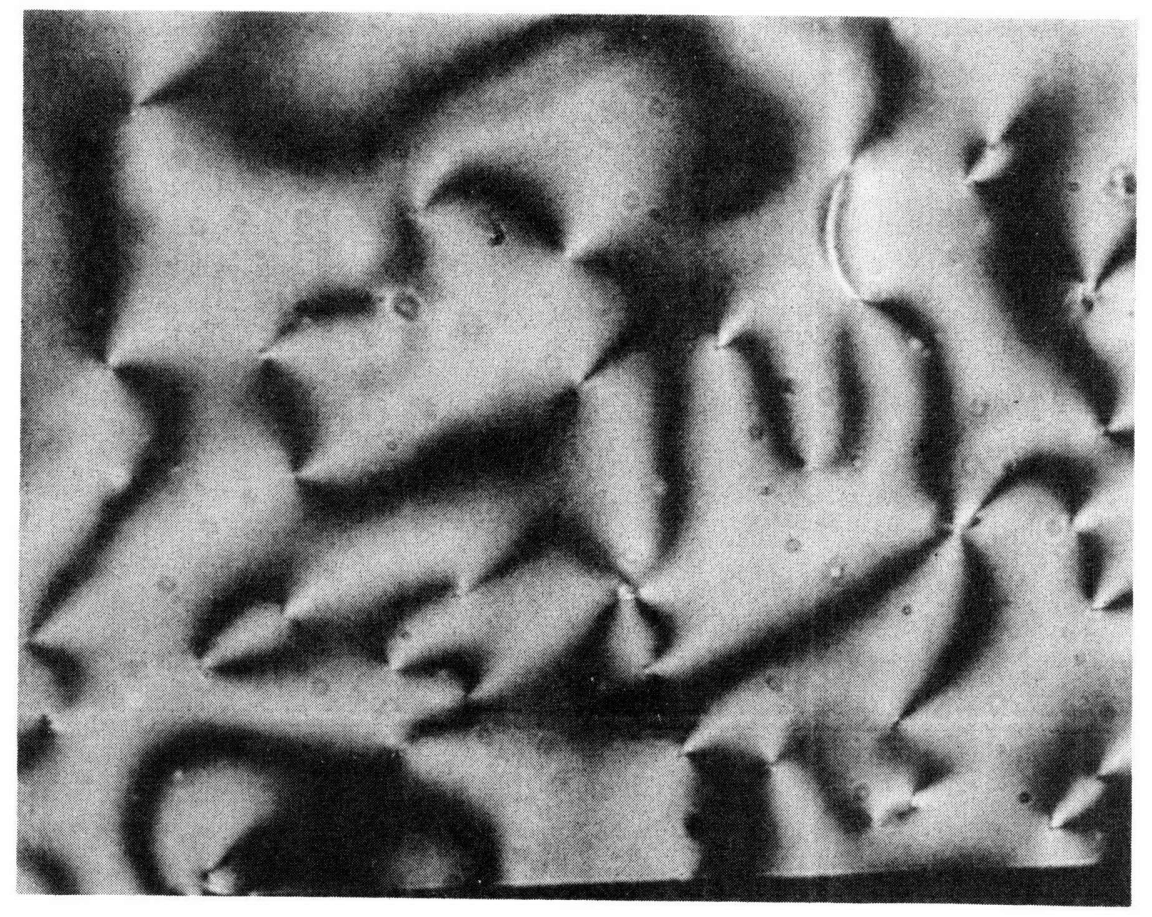

Fig. 4-5. An optical micrograph is shown of a $50 \mu \mathrm{m}$ thick slab of MBBA in the nematic phase confined between smooth amorphous substrates. The slab is observed between crossed polarizers. Several wedge disclinations are visible. 
The rapid variations in intensity indicate essentially random orientation of the nematic director in the plane in this region. Within region $C$, transmission nulls were obtained when the incident polarization was precisely parallel or perpendicular to the grating direction to within the experimental error of \pm .25 degrees. This evidence indicates that within region $C$ the projection of the nematic director was uniformly aligned either parallel or perpendicular to the grating groove direction. Uniform alignment was observed in a1l areas of region $C$. At the border between regions $A$ and $C$ the uniform alignment of region $C$ abruptly changed to the random alignment of region $A$. In the intermediate region, $B$, alignment was not uniform, yet it was generally in the direction of the grating. Further experiments were performed to determine more precisely the orientation of the director. The MBBA in the sandwich was lightly doped with a dye whose elongated molecule is known to orient parallel to the liquid crystal director ${ }^{62}$. It is also known that this dye preferentially absorbs light polarized parallel to the dye molecule's long axis. Within region $C$ the doped MBBA was found to preferentially absorb light polarized parallel to the groove direction. Thus it was concluded that the projection of the director was definitely oriented parallel to the grooves. To determine whether the director was parallel to the plane of the substrates or tilted with respect to the plane the phase delay for a normally incident wave was measured. Within experimental error the director was found to be parallel to the substrate plane. Because of the large uncertainty of $\pm 15^{\circ}$ associated with the measurement technique it is possible that there could be a slight tilt of the director from the plane. The analysis presented in section 4.3 
predicts the observed orientation. This is seen by noting that orientations of the liquid crystal in the sandwich will correspond to the equilibrium orientation determined by the minimum free energy configuration. The contributions to the free energy will include the interfacial free energies associated with the grating structures and the free energy of the liquid crystal layer between the grating structures. As argued in section 4.3 the minimum interfacial free energy for MBBA on a square grating should occur for $\vec{n}$ parallel to the groove direction since this minimizes both the "physiochemical" and distortion parts of the interfacial tension for MBBA. Minimum free energy for the bulk nematic phase obviously occurs for constant $\vec{n}$. Thus the equilibrium orientation of MBBA in region $C$ of the sandwich should indeed correspond to $\vec{n}$ parallel to the groove direction. After the alignment experiments with MBBA were completed, the sandwich was disassembled and the MBBA was removed from the substrates by immersion in acetone, followed by UV ozone cleaning ${ }^{44}$. 


\subsubsection{ALIGNMENT OF M-24 IN THE NEMATIC AND SMECTIC A PHASES}

An experiment similar to the one described above for MBBA was undertaken using the liquid crystal M-24 (see appendix D), which has a smectic A phase as well as a nematic phase. A sandwich was constructed as shown in Fig. 4-4 in the manner described in section 4.4.2. In this case, Mylar spacers $25 \mu \mathrm{m}$ thick were used rather than Teflon. The sandwich was heated to above the isotropic transition of $M-24$ on a temperature-stabilized microscope hot stage. The liquid crystal was then introduced between the substrates by capillary action. The sample was next cooled, very slowly, to the nematic-isotropic transition at $78.3{ }^{\circ} \mathrm{C}$ while being observed between crossed polarizers. When the transition occured, a small number of disclinations appeared and then shrank very rapidiy (in less than one second) and disappeared. In the nematic phase the three regions (see section 4.4 .2 and Fig. 4-4) A, B, and $C$ as observed between crossed polarizers in transmitted light had very distinct properties. In region $C$, nulls in the transmission wera observed for polarization precisely parallel or perpendicular to the grating grooves. In region $A$, the orientation of the polarizers with respect to the sample at which the transmission null occured wandered randomly from area to area. In region $B$, the orientation of the nulls varied only slightly from the grating direction. The interpretation of this result is the same as given in section 4.4 .2 for MBBA. The absence of disclinations in all regions in this case was particularly striking, however. This could possibly be explained by the very slow cooling of the M-24 through the nematic-isotropic transition which would allow oriented 
"nucleation and growth" (see Chapter 5) of the nematic phase from the isotropic phase.

After observing the M-24 sandwich in the nematic phase, it was cooled very slowly $\left(<10^{-1}{ }^{\circ} \mathrm{C} /\right.$ minute $)$ through the nematic to smectic $\mathrm{A}$ transition at $66.5^{\circ} \mathrm{C}$. Within region $\mathrm{C}$ the only observed change below the transition was the absence of director fluctuations which is characteristic of the smectic phase. The entire area of region $C$ appeared uniformly dark between crossed polarizers for the groove direction parallel or perpendicular to the incident polarization. However, below the transition, region $A$ had a remarkable appearance. Observed between crossed polarizers it presented a kaleidoscopic pattern of dislocations and focal conic textures ${ }^{5}$. Region B exhibited a striated texture of dislocations, but the average director orientation coincided with the grating direction. The transition was slowly traversed in temperature several times with the result that the texture in regions $A$ and $B$ changed slightly: The other features of the phenomenon were unchanged.

The temperature of the sandwich was then lowered $3^{\circ} \mathrm{C}$ below the nematic-smectic A transition. As the temperature decreased very faint striations could be observed in region $C$. These are believed to be dislocations in the smectic phase ${ }^{5}$ caused by defects at the edge of region $c$.

Once again the observed orientation of the liquid crystal by the square-wave surface-relief structure can be explained by the minimization of free energy arguments given in sections $2.4,4.2,4.3$, and 4.4.2. 


\subsection{CONCLUSIONS}

An experiment has been done where a well controlled and characterized surface relief structure was fabricated and shown to align both nematic and smectic liquid crystals with the orientation consistent with theory. Berreman $^{56}$, Kahn ${ }^{48}$, and others ${ }^{57}$ have attempted to explain the observed orientation of nematic liquid crystals by rubbed and evaporated surfaces with a theory of liquid crystal orientation by surface-relief structures. Their efforts have been frustrated by the difficulty of observing and characterizing the surfaces. Here a different approach has been taken and a theory similar to theirs has been shown to be qualitatively correct. It is worth noting that Berreman's theory was formulated to explain the specific case of liquid crystal alignment and that it is only a specific case of the broader theory of crystalline overlayer orientation by surface relief structures which is presented here. Further liquid crystal orientation experiments are suggested in Chapter 6. 
V. ORIENTATION OF SOLID CRYSTALLINE OVERLAYERS BY SURFACE RELIEF STRUCTURES ON AMORPHOUS SUBSTRATES

\subsection{INTRODUCTION}

In this chapter the orientation of solid crystalline overlayers on amorphous substrates by surface-relief structures is discussed. The general features of thin solid film growth are first presented to provide background. Specific models are then discussed for the growth processes of nucleation, island information, and coalescence. From the models of the individual steps in the growth process, a model is constructed for oriented thin film growth on a surface.relief structure on an amorphous substrate. Finally, experimental results, which agree with this model,are presented showing oriented growth of $\mathrm{KCl}$ on squarewave grating structures on amorphous $\mathrm{SiO}_{2}$. The results of gold and tin depositions on square-wave gratings are also presented and explained in light of the growth model. 


\subsection{GENERAL FEATURES OF THIN FILM GROWTH}

\subsubsection{THE THIN FILM GROWTH PROCESS}

The deposition of thin films on solid substrates has been the subject of extensive research. A large number of deposition techniques have been developed including evaporation, sputtering, chemical vapor deposition, molecular beam, and liquid phase growth techniques. Many substrate-deposit combinations have been investigated. Several comprehensive reviews of this subject have recently been published, $1,2,63$

Despite the great variety of deposition techniques and substrate and deposit combinations, the general features of the mechanism of film formation is similar in most cases (a possible exception is the case where the deposit-substrate bonding is very strong and layer by layer growth occurs). The several distinct stages of thin film growth are illustrated in Fig. 5-1. In the initial stage of deposition a large number of small nuclei form on the substrate. These nuclei often have a multiplicity of crystallographic orientations regardless of the substrate crystalline structure. On a smooth substrate the nuclei are randomly distributed, however, an extremely interesting instance of nonrandom nucleation is sometimes seen on cleavage steps and other substrate topographic features. An example of this phenomenon (from work of Basset's ${ }^{64}$ ) termed "decoration" is shown in Fig. 5-2. (Note that the tiny gold nuclei have preferentially nucleated on the $\mathrm{NaCl}$ cleavage steps.) 


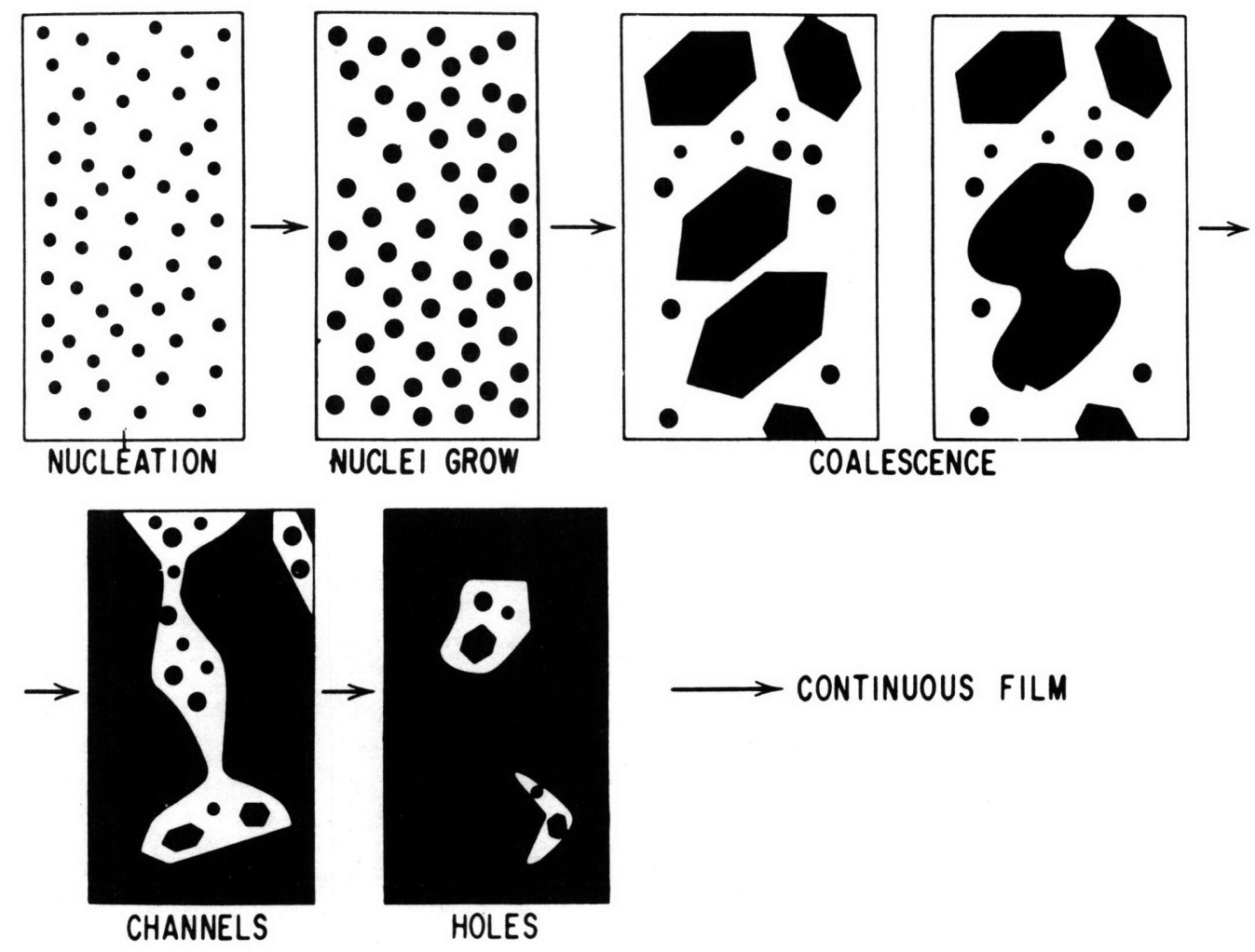

Fig. 5-1. A shematic illustration of the distinct stages of thin film growth. This sequence of film growth is characteristic of a large number of deposit-substrate combinations where the deposit-substrate bonding is weaker than the deposit-deposit bonding. 


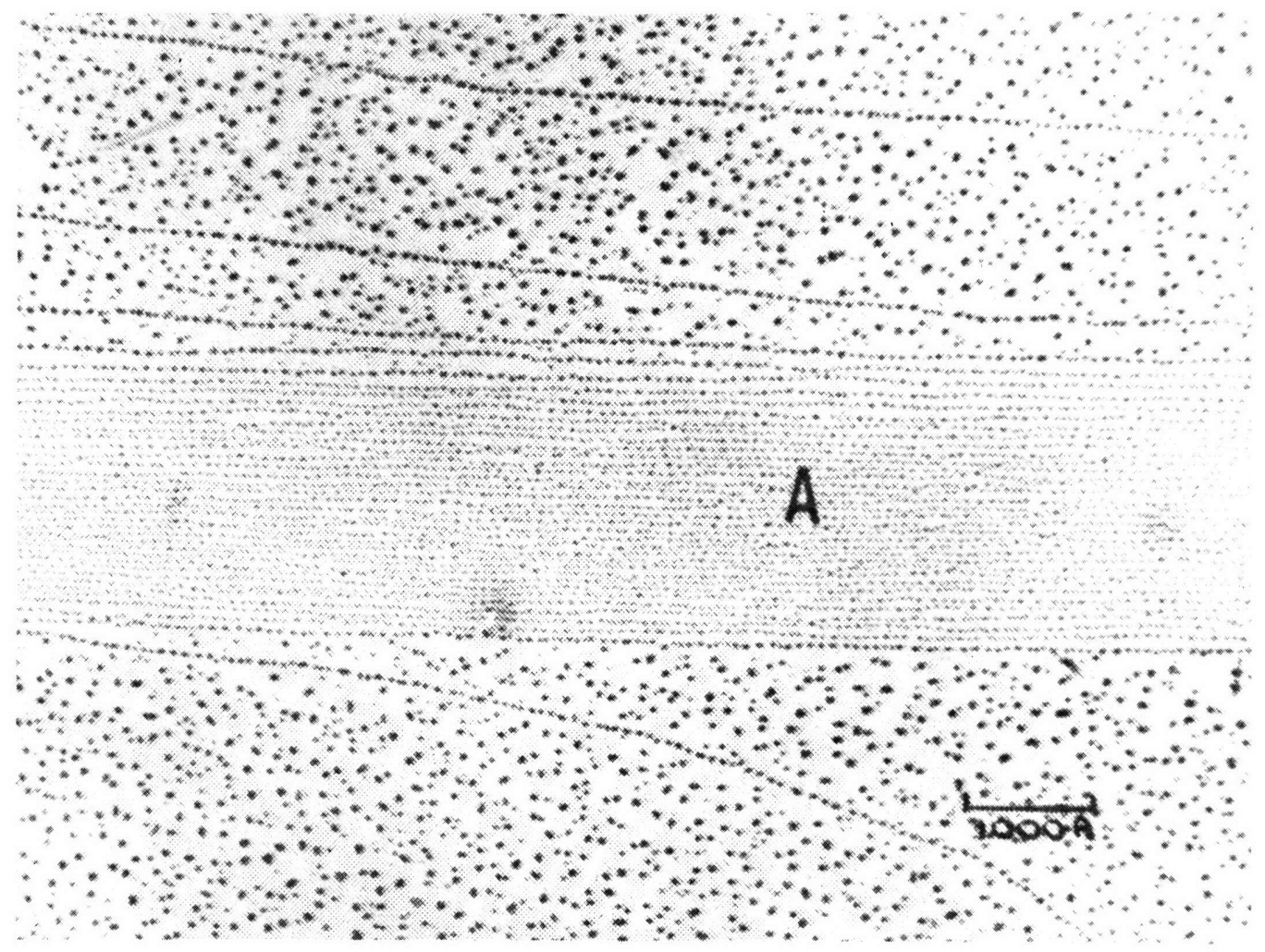

Fig. 5-2. Transmission electron micrograph of a gold deposit on rocksalt at very low average film thickness. Note that the natural cleavage steps have been "decorated" with gold nuclei and that in the region where the steps are closely spaced, the nuclei are completely confined to the steps. (Bassett et al.) 64 
Following nucleation, the nuclei or "islands" grow in size. In some cases, those with a particular crystallographic orientation grow more rapidly than others. It also appears that these small islands often have considerable mobility on the substrate surface and can easily change their crystallographic orientation.

Eventually, as growth continues, some islands coalesce with one another to form larger islands. During coalescence of two isolated islands, there is a considerable amount of mass transfer, and a tendency for the compound island to take on a single crystallographic orientation. It is often at this stage that the formation of large area single crystal islands takes place through the reorientation of many small islands. It has been observed that in some cases the islands exhibit a liquid-like behavior during coalescence. ${ }^{65}$ Explanations of the phenomenon of coalescence attribute this behavior to mass transfer by surface selfdiffusion of the deposit atoms driven by the tendency of the small islands to minimize their surface free energy. The process of coalescence and recrystallization of islands continues until the growing islands reach a size at which coalescence is inhibited and a more or less continuous network of islands is formed. Once this "network" stage has been reached, recrystallization is inhibited. The voids or channels that still exist between the joined islands are usually quite irregular in shape. These become filled in as small islands located in the channels grow and coalesce with the network structure. The size of the islands just prior to the network stage can vary greatly with temperature, 
substrate-deposit combination, and deposition conditions. If a continuous film is formed at a very small island size and the islands are randomly oriented, an amorphous or small grained polycrystalline film results. This is the case with most depositions done at low temperatures (relative to the deposit melting point) and high deposition rates on both amorphous and single crystal substrates. At higher temperatures and lower deposition rates the islands tend to be larger at the network stage because of the lower nucleation rate and increased mobility of the deposit. Island sizes of $100 \mathrm{~nm}$ or more are not uncommon. If these large islands are randomly oriented, a large grained polycrystalline film is formed. If the islands have a single or highly preferred orientation at the network stage, a single crystal heteroepitaxial film can result.

Heteroepitaxy is known to occur only on single crystal substrates and for a 1 imited number of substrate-deposit combinations. Because of the complexity of the problem and the large number of often uncontrolled and unknown parameters involved no.generally successful theory of heteroepitaxy has been developed and there is a great deal of debate about the details of the mechanisms which lead to heteroepitaxy.

Al though heteroepitaxy does not occur on amorphous or polycrystalline substrates ${ }^{*}$, the polycrystalline films deposited on smooth amorphous substrates are not necessarily entirely random. In fact, under the proper deposition conditions, films with a "fiber texture" can be grown. *With the possible exception of some very controversial work by G. Distler, where it has been claimed that single crystal films have been grown on "replicas" of single crystal substrates in amorphous materials. 
where a single crystallographic direction of the individual grains has a highly preferred direction. This phenomenon is discussed in detail in section 5.2.7.

The above general features of thin film growth appear to be well understood. It is also widely known that the surface structure of a single crystal substrate can dramatically affect the final quality of a heteroepitaxial film. Poorly polished substrates with many scratches and other defects always tend to produce inferior films. However, the influence of the specific structures on a substrate surface on the growth process has received little attention aside from a few studies of decoration of cleavage steps. The uncontrolled nature of the surfaces prepared by conventional techniques makes systematic study of these effects very difficult. Thus researchers have endeavored to eliminate the uncontrolled influence of accidentally produced surface structures by producing a featureless smooth substrate. (An interesting exception to this general rule occurs in homoepitaxial or layer by layer heteroepitaxial film growth where the substrate plane is cut slightly off the desired growth plane such that the surface consists of an array of monotomic steps). Even less is known about the influence of surface topography on film growth on amorphous substrates.

To understand how artificially-produced surface-relief structures on an amorphous substrate might influence the thin film growth process a detailed understanding of each of the distinct stages of thin film growth process is useful. Each of these growth stages is discussed in the 
next sections and a model of thin film growth on surface-relief structures is presented in section 5.3 . 


\subsubsection{EQUILIBRIUM FORM OF A CRYSTALLINE ISLAND ON AN AMORPHOUS SUBSTRATE}

During the nucleation, island growth, and coalescence stages of thin film growth the deposit exists in the form of small isolated islands. Insight into the behavior of these islands can be obtained by considering the equilibrium form of a crystalline material on a substrate.

The shapes of large growing crystals are, in most cases, determined by competition between the rates of various transport mechanisms and a complete understanding of the development of crystal shape requires a detailed knowledge of the kinetics of growth. Fortunately, the small size of the islands involved in thin film growth (usually less than $200 \mathrm{~nm}$ ) makes an equilibrium treatment of the problem realistic since for small islands the tendency of the crystal to lower its surface free energy is often the principle motivation for changes in surface structure. A partial treatment of the problem of determining the equilibrium form of a small crystalline island has been given by Winterbottom. The following development closely parallels his arguments.

Three interfacial tensions are involved, $\gamma_{s v}, \gamma_{s d}, \gamma_{d v}$, the substrate-vapor, substrate-deposit, and deposit-vapor. Under conditions of constant volume, temperature, and total number of moles, the equilibrium configuration is determined by equation 2.5. More explicitly this requirement becomes

$$
\delta \int_{s v} \gamma_{s v} d A_{1}+\delta \int_{s d} \gamma_{s d} d A_{2}+\delta \int_{d v} \gamma_{d v} d A_{3}=0
$$


For a constant deposit volume, where $\delta$ specifies a variation of the surface shape and the integrals are taken over the entire interfacial area of the specified interface.

If it is assumed that the substrate surface is not deformed by the deposit, $-d A_{1}=d A_{2}$ and expression 5.1 can be simplified to yield

$$
\delta \int_{s d}\left(\gamma_{s d}-\gamma_{s v}\right) d A_{1}+\delta \int_{d v} \gamma_{d v} d A_{3}=0
$$

$\mathrm{OR}$

$$
\delta \int_{d} \gamma^{*} d A=0
$$

Where the integral is taken over the entire deposit surface and $\gamma^{\star}$ is an interfacial tension for the deposit defined by

$$
\gamma^{*}=\left\{\begin{array}{l}
\gamma_{\mathrm{dv}} \text { for the deposit-vapor interface } \\
\gamma_{s d}-\gamma_{s v} \text { over the substrate-deposit } \\
\text { interface }
\end{array}\right.
$$

If the deposit-substrate orientation is specified, the function $\gamma^{*}$ is unique. Equation 5.3, subject to the volume constraint, can then be solved directly by the calculus of variations. Landau and Lifshitz have obtained a general solution to this problem which has a simple geometric interpretation known as the Gibbs-Wulff construction. The solution for the equilibrium form of a cubic deposit material with $a^{\prime}\{100\}$ face parallel to an amorphous substrate is illustrated in Fig. 5-3. First the polar plot of $\gamma^{*}$ is drawn, the Gibbs-Wulff construction then consists of planes drawn perpendicular to each radius vector at the 
$18-8-14542$
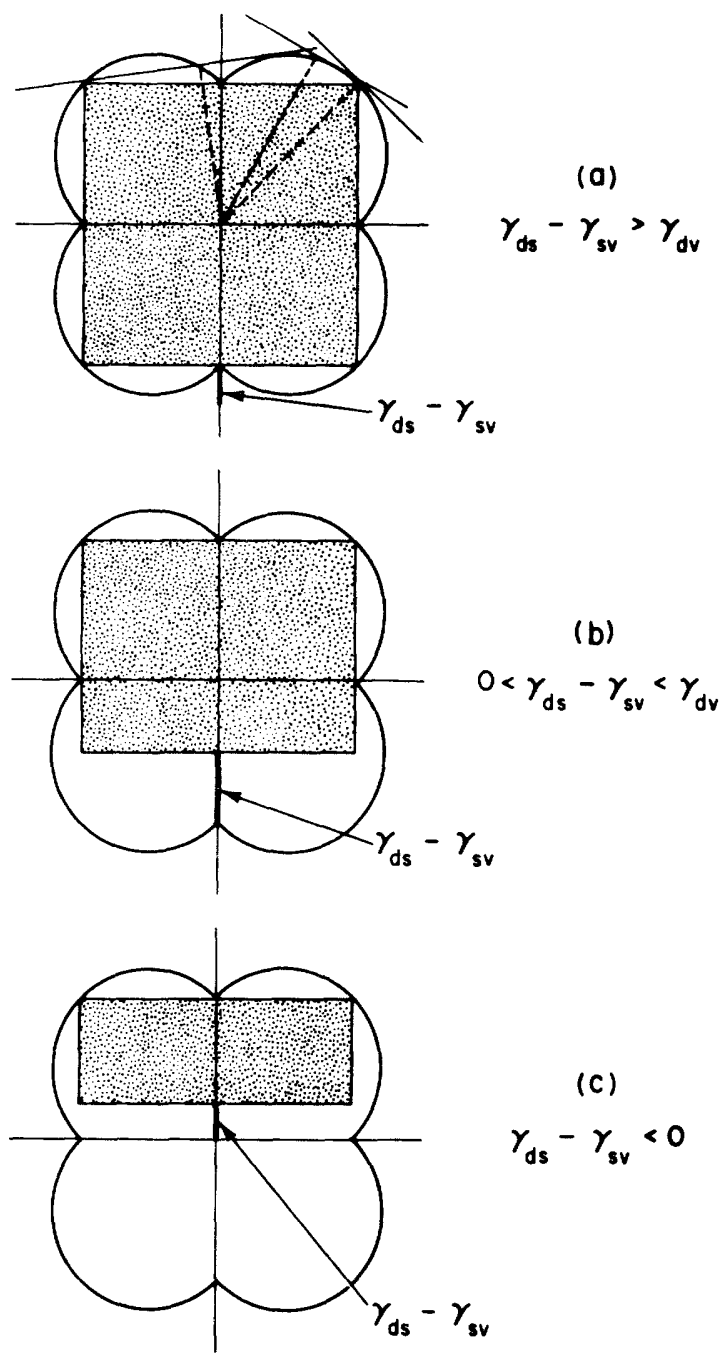

Fig. 5-3. An illustration of the application of the Gibbs-Wulff construction to the determination of the equilibrium form of a cubic deposit on a substrate. The equilibrium form is geometrically similar to the inner envelope of all possible planes drawn normal to the $\gamma(\vec{n})$ (Wulff) plot. 
$\gamma^{*}$ surface. The inner envelope formed by all possible planes is geometrically equivalent to the equilibrium form of the deposit. Three cases are shown in Fig. 5-3 where $\gamma_{s d}-\gamma_{s v}$ is $<0,>0$, and $>\gamma_{d v}$. Note that the case $\left(\gamma_{s d}-\gamma_{s v}\right)<0$ can be handled by placing the origin (Wulff point) outside the envelope of the construction. (Insight into the equilibrium forms of various deposits on flat substrates can be gained by considering the possible equilibrium shapes of free particles of anisotropic and crystalline materials. From the possible shapes of the $\gamma$ plot as enumerated in Table 2-1, representative equilibrium shapes ${ }^{8}$ can be drawn as shown in Fig. 5-4. Flat faces are obtained only if the $\gamma$ plot is cusped. (Note that a polyhedral shape is obtained only in the case of a cusped $\gamma$ plot with a large anisotropy.) The Gibbs-Wulff construction shown in Fig. 5-3 does not give the absolute minimum free energy configuration, it simply gives the minimum free energy configuration for a given substrate-deposit orientation. To determine the absolute minimum of free energy, variations of the substrate-deposit interfacial orientation must also be considered. However, it is easily seen that if the function $\gamma_{s d}-\gamma_{s v}$ is cusped at some orientation there is a relative minimum of free energy at the orientation of the cusp. At the cusp with minimum $\gamma_{s d}-\gamma_{s v}$ an absolute minimum of free energy should be obtained. If we assume that the interfacial tension varies for a cubic deposit. an an amorphous substrate as shown in Fig. 2-1(c). then the equilibrium form determined by the modified Gibbs-Wulff construction in Fig. 5-3 will represent the absolute minimum of free 


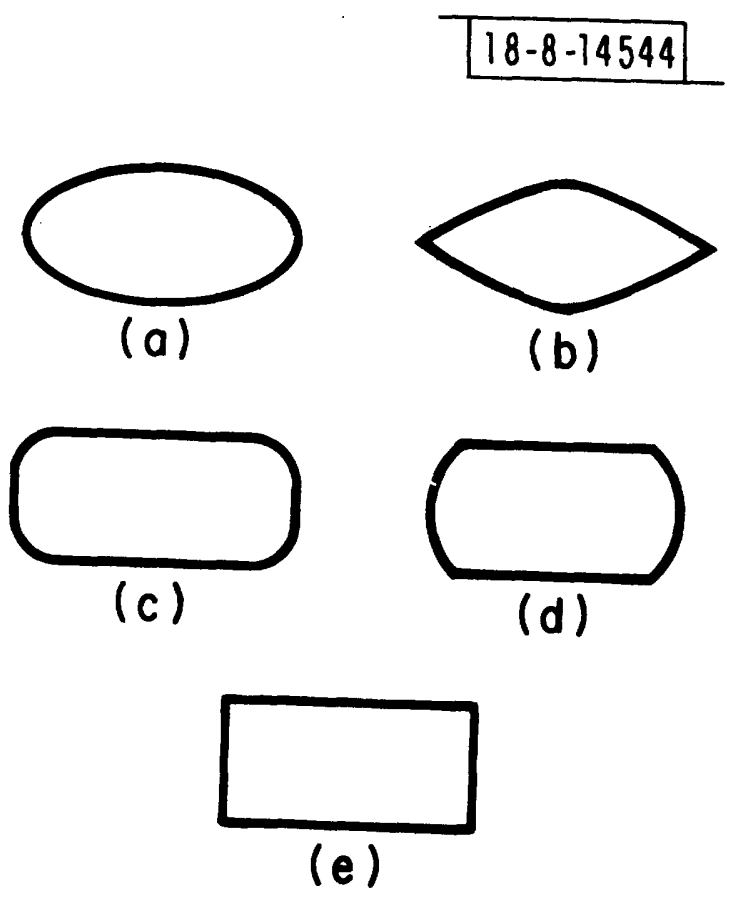

Fig. 5-4. A schematic representation of the equilibrium forms for the possible interfacial tensions as enumerated in Table 2-1. 
energy for the system and will be the true equilibrium form.

The modified Gibbs-Wulff construction can also be used to find the equilibrium form of a deposit island at a step for a specified deposit orientation relative to the step. The required construction is illustrated in fig. 5-5 for both an isotropic and a cubic material. Once again the equilibrium form for the cubic material shown in Fig. 5-5 will correspond to a true equilibrium for the system because both deposit-substrate interfaces occur at cusped minimum when $\gamma_{d s}$ varies as in Fig. 2-1(c).

In general to find the minimum free energy configuration for a deposit contacting a nonplanar substrate the full variational problem must be solved. 


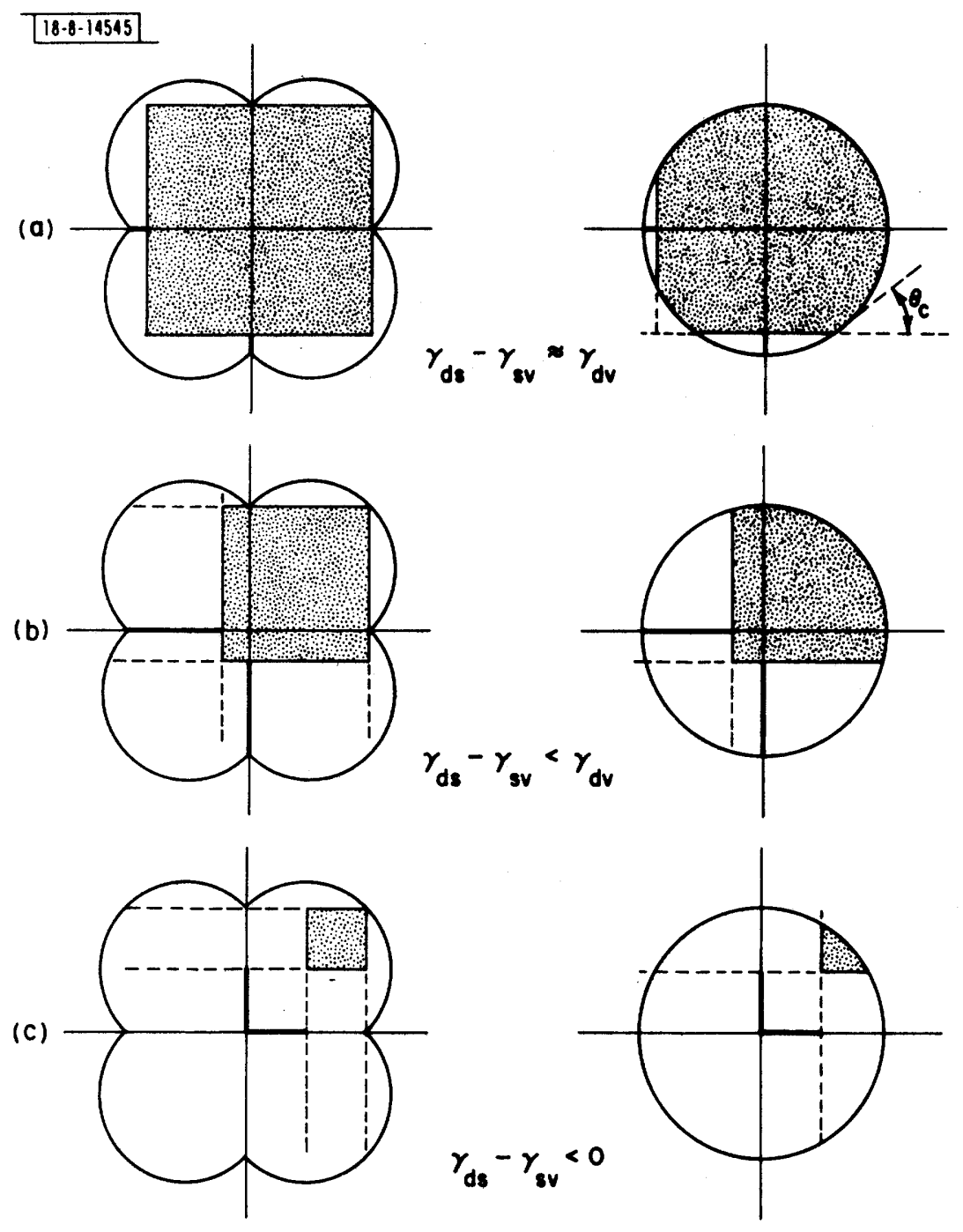

Fig. 5-5. The shaded area of the above diagrams are cross-sections determined by the Gibbs-Wulff construction of the equilibrium form of a cubic crystalline material and an isotropic material in a $90^{\circ}$ corner of an isotropic material. The cross-section is taken in a plane which is perpendicular to both faces of the $90^{\circ}$ corner. 


\subsubsection{NUCLEATION}

Both classical thermodynamic and atomistic theories have been developed to explain nucleation. The theory of nucleation on a foreign substrate has been treated extensively in a recent review. 71 The classical theory is considerably less involved than the atomistic theory, and the problem can be handled with a smaller number of variables. The crucial assumption in the classical theory is that small cluster of atoms can be characterized by the same thermodynamic properties as those of the stable bulk phase. Nuclei are thus assumed to have an interfacial tension, $\gamma$, and a free energy of formation per unit volume, $\Delta G_{v}$, identical to that of the bulk phase. If the nuclei are sufficiently large, this appears to be a reasonable assumption, for very small nuclei the use of macroscopic thermodynamics is highly questionable. In any case the analysis presented here is able to provide only a qualitative understanding of the nucleation phenomenon. Quantitative comparisons with theory could not be made in this study due to the lack of accurate knowledge of even the bulk values of the thermodynamic quantities. The treatment given below follows closely the work of Robertson and Pound, ${ }^{71}$ Kenty and Hirth, ${ }^{72}$ and Chakraverty and Pound. ${ }^{73}$

The mechanism of formation of nuclei is as follows. Atoms from the parent phase, which is supersaturated with respect to the bulk solid phase, strike the surface and either immediately rebound or are absorbed and become thermally equilibrated with the substrate. These absorbed atoms (adatoms) diffuse over the surface and through 
fluctuations in concentration, form small clusters of atoms which grow or decay by the addition or loss of atoms. Increase in the size of a cluster is energetically unfavorable until it exceeds a critical size, at which time it grows rapidly, limited only by the arrival of adatoms.

Energy to drive the nucleation process comes from the volume (Gibbs) free energy change, $\Delta G_{v}$, for the transformation from the parent phase to the solid phase given by;

$$
\Delta G_{v}=\frac{-K T}{v} \ln \frac{n_{1}}{n_{1}}
$$

where, $u$, is the atomic volume and $n_{1} / n_{1} e$ is the super saturation where $n_{1}$ is the actual adatom concentration and $n_{1} e$ is the adatom concentration which would exist in equilibrium with the solid phase.

The "barrier" to nucleation is provided by the interfacial free energy associated with the nuclei. If the equilibrium form of a nucleus is known, the density of free energy of formation, $\Delta G_{v t}$, and the total interfacial free energy density, $\gamma_{t}$, can be calculated for a nucleus of unit size, $r$. The free energy of formation $\Delta G_{f}$ of a nucleus will then be:

$$
\Delta G_{f}=\Delta G_{v t} r^{3}+\gamma_{t} r^{2}
$$

The critical size of the nucleus, $r^{*}$, will correspond to the size at which the maximum value of the free energy of formation, $\Delta G_{f}$, occurs since growth will be energetically favorable for a nucleus larger than $r^{*}$. Maximizing equation (5.6) yields $r^{*}=-\frac{2}{3} \frac{\gamma_{t}}{\Delta G}$

Although not numerically equal the interfacial tension and interfacial free energy will have the same symmetry properties. For purposes of discussion, here they will be assumed equal. 
and

$$
\Delta G_{f}^{*}=\frac{4}{27} \quad \frac{\gamma_{t}^{3}}{\Delta G_{v t}} 2
$$

Since $\Delta G_{v t}$ depends upon supersaturation (see equation 5.5 ) one sees that $r^{*}$ can be large if the supersaturation is low.

The steady state nucleation rate, $J$, with units of number of nuclei formed $/ \mathrm{cm}^{2} / \mathrm{sec}$ has been formulated as:

$$
\begin{aligned}
& \text { been formulated as: } \\
& J=n_{0} A \text { e } \times p\left(\frac{-\Delta G_{f}^{*}}{K T}\right)
\end{aligned}
$$

where $n_{0}$ is the number of nucleation sites $/ \mathrm{cm}^{2}$ and $A$ is an adatom impingement frequency which is expected to change only slightly for the variations in nuclei geometry considered in this analysis, and will be considered to be independent of such factors. It is obvious from equation (5.9) that the nucleation rate decreases rapidly as the critical free energy of formation $\Delta G_{f}{ }^{*}$ increases.

An important quantity which can be obtained from equation (5.9) is the relative nucleation rate of two nuclei with slightly different critical free energies of formation, $\Delta G_{f 1}{ }^{*}$ and $\Delta G_{f 2}$ such that $\Delta G_{f 1}-\Delta G_{f 2}=\Delta g^{*}$. From equation (5.9) the ratio of their nucleation rates will then be:

$$
\frac{\mathrm{J}_{1}}{\mathrm{~J}_{2}}=\mathrm{e} \times \mathrm{p}\left(-\frac{\Delta \mathrm{g}^{*}}{\mathrm{KT}}\right)
$$

where the number of nucleation sites is assumed equal for both nuclei.

The implications of equation (5.10) will be discussed in the context of the observed nucleation phenomena of decoration and fiber texture in 


\section{sections 5.2.4 and 5.2.7.}

For the case of deposition from a vapor, relative nucleation rate equation (5.10) has been discussed in great detail by Kenty and Hirth. Their basic conclusion is that even for very small $\Delta \mathrm{g}^{*}$ the lower free energy nuclei can be highly preferred under conditions of low supersaturation (meaning in the case of vapor deposition, high substrate temperatures and/or low impingement rates). The size of the critical nucleus, calculated using equation 5.7, for deposition from a vapor tends to be less than 1 nanometer under most deposition conditions. Nucleation experiments ${ }^{2}$ by Chopra indicate that the size of the critical nucleus is less than three atoms for metals deposited on amorphous carbon. It appears that equation 5.7 overestimates the size of the critical nucleus in the case of small critical nuclei. 


\subsubsection{DECORATION EFFECTS}

One of the most dramatic examples of the influence of the substrate surface relief structure on thin film growth is the decoration of natural cleavage steps at the nucleation stage. This effect, first described by 64 Basset, has mainly been used to make surface defects of single crystals visible in transmission electron microscopy. An example of the phenomenon shown earlier in Fig. 5-2. shows small gold nuclei which have nucleated preferentially on natural cleavage steps on a $\mathrm{NaCl}$ surface. (A remarkable feature of this particular example is that all of the nuclei are confined to the steps in the region where the steps are closely spaced).

Decoration has been observed on natural cleavage steps of a great many deposit-substrate combinations, including gold-on-graphite ${ }^{74}$, goldon-rocksalt $t^{64}$, silver-on-mica ${ }^{75}$, and tin-on-rocksalt ${ }^{76}$. In the case of gold-on-rocksalt it has been shown conclusively that even monatomic cleavage steps can be decorated.

Preferential nucleation at steps (where the number density of nuclei is greater in the region of the step than on a flat area) has been explained with both classical nucleation theory and an atomistic theory. The classical theory explanation can be illustrated very easily using the results derived in sections 5.2 .2 and 5.2.3.

Evidently the nucleation rate must be higher at the steps than on a flat region of the substrate if decoration is to be observed. To find the nucleation rate ratio on the flat part of a substrate versus 
at a step on the same substrate one can use equation (5.10) if the difference in free energy of the critical nuclei at the two sites is known. This difference, $\Delta \mathrm{g}^{\star}$, can be found by first determining the equilibrium form of the nuclei at a step and on a plane using the modified Gibbs-Wulff construction detailed in section 5.2.2. The free energy of formation of the critical nucleus is then found by maximizing $\Delta G_{f}$ for the equilibrium shape. $\Delta g^{*}$ is then the difference between the critical free energy of formation at the step and at the plane.

The equilibrium forms of nuclei on a plane and at a step for a material with a cubic interfacial tension are illustrated schematically in Fig. 5-6.

The corner of the step in Fig: 5-6 has a finite radius, $R_{a}$. If the critical radius $r^{*}$ is large compared to $R_{g}$, the step can be regarded as a perfect 90 degree step, if $r^{*}$ is comparable to $R_{g}$ or smaller the problem will reduce to a problem of nucleation on a concave surface of radius $R_{g}$, versus nucleation on a plane. This point is discussed further in section 5.3 for the case of decoration of artificial surface relief structures. In the following discussion it is assumed that $r^{*} \gg R_{g}$. The critical nucleus free energy difference, $\Delta g_{i}{ }^{*}$ for a nucleus with isotropic interfacial tensions calculated from the equilibrium form given by the Gibbs-Wulff construction of Fig. , 5-5 is found to be:

$$
\underset{i}{\Delta g^{*}}=\frac{4 \pi}{3} \frac{\gamma_{d v}{ }^{3}}{\Delta G_{v}{ }^{2}} \quad\left(F\left(\theta_{c}\right)\right)
$$




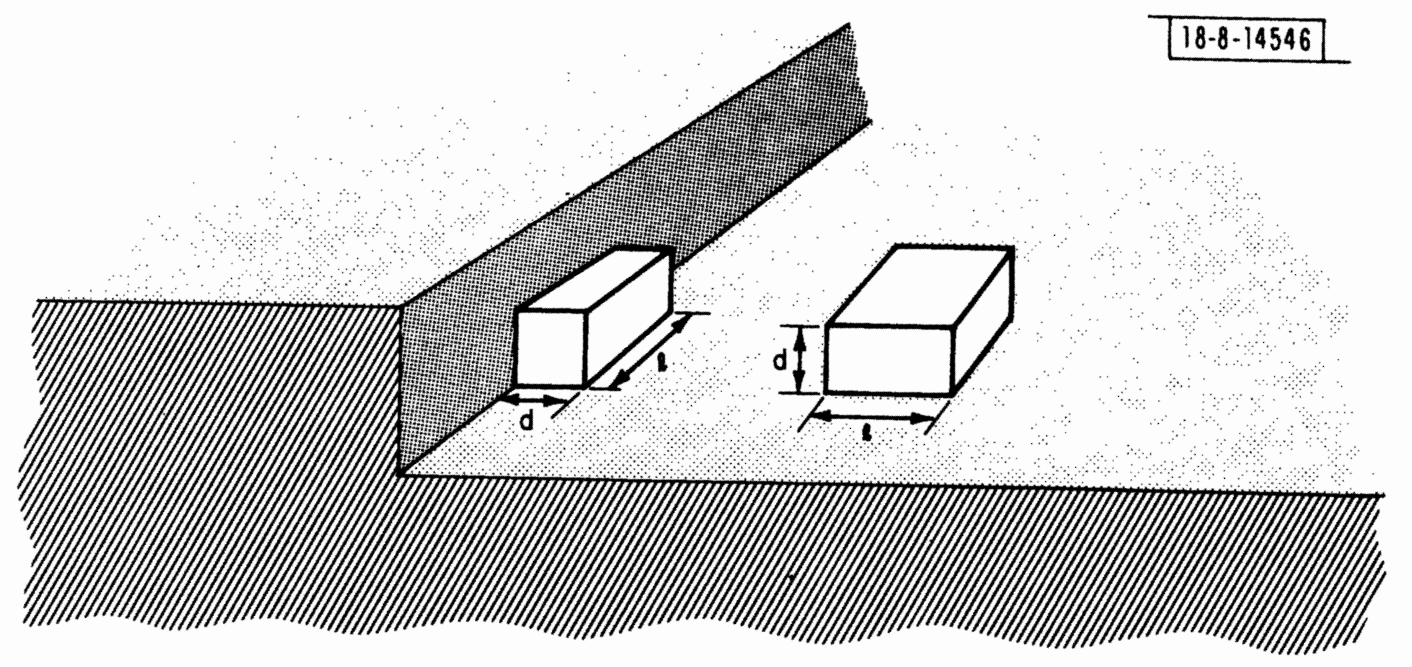

Fig. 5-6. The equilibrium forms of a deposit at a step and on a plane are shown for a deposit material where the interfacial tensions are the same as those used to construct Figures $5-3(b)$ and $5-5(b)$. 
where $F(\theta)$ is a function of the contact angle $\theta_{c}$, Where contact angle is defined as $\theta_{c}=\cos ^{-1}\left(\frac{\gamma_{d s}-\gamma_{s v}}{\gamma_{d v}}\right)$. From equation (5.10), the nucleation ratio $\frac{I_{s}}{I_{f}}$, for a nucleus with isotropic interfacial tension will be:

$$
\ln \left(\frac{I_{s}}{I_{f}}\right)=\ln \left(\frac{n_{s}}{n_{f}}\right)+\frac{\Delta g_{i}^{*}}{K T}
$$

Equation (5.12) is plotted versus contact angle in Fig. 5-7 using experimentally determined values of $\gamma_{d v}, \frac{n_{s}}{n_{f}}$, and $\Delta G_{v}$. (The shape of the curve does not depend on these parameters).

The critical nucleus free energy difference $\Delta g^{*}$, for a cubic nucleus is found to be:

$$
\Delta g_{c}^{*}=\frac{4}{3} \frac{\gamma_{d v}^{3}}{\Delta G_{v}{ }^{2}}\left(k-k^{2}\right)
$$

The ratio of nucleation rates at a step and on a plane for the case of the cubic nucleus, $\frac{J_{S}}{J_{f}}$, is plotted in Fig. $5-8$ as a function of $K$. Where $K=\frac{d}{l_{i}}$ where $d$ and $\ell$ are defined in Fig. 5-6.

Several important qualitative features of the decoration effect are apparent from equations (5.12) and (5.13) and Figures 5-7 and 5-8. It is apparent that the effect is greatly enhanced for low supersaturations, or equivalently low $\Delta G_{v}$. It is also seen that the ratio of nucleation rates becomes small as the substrate nuclei interaction becomes negligible (i.e., $\theta_{C} \rightarrow 180^{\circ}$ or $K \rightarrow 1$. It is also worth noting that the nucleation 


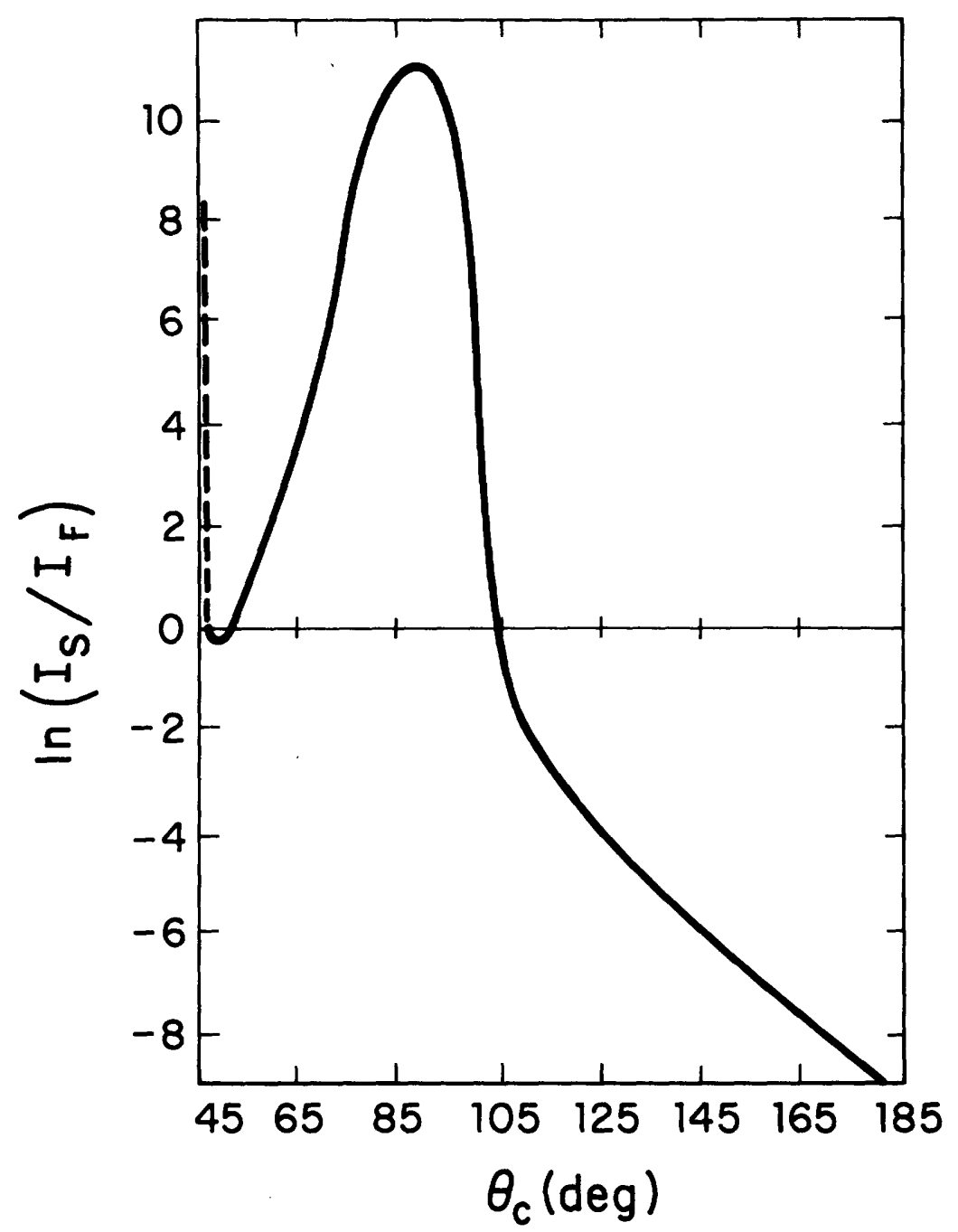

Fig. 5-7. A plot ${ }^{73}$ of the calculated ratio of nucleation rate at a step, $I_{s}$, and on a flat plane, $I_{f}$. versus nuclei contact angle $\theta_{c}$. Plotted for $n_{s} / n_{f}=10^{-4}$ and $(4 / 3) \gamma_{d v}^{3} /\left(\Delta G_{v}^{2} K T\right)=20$. 


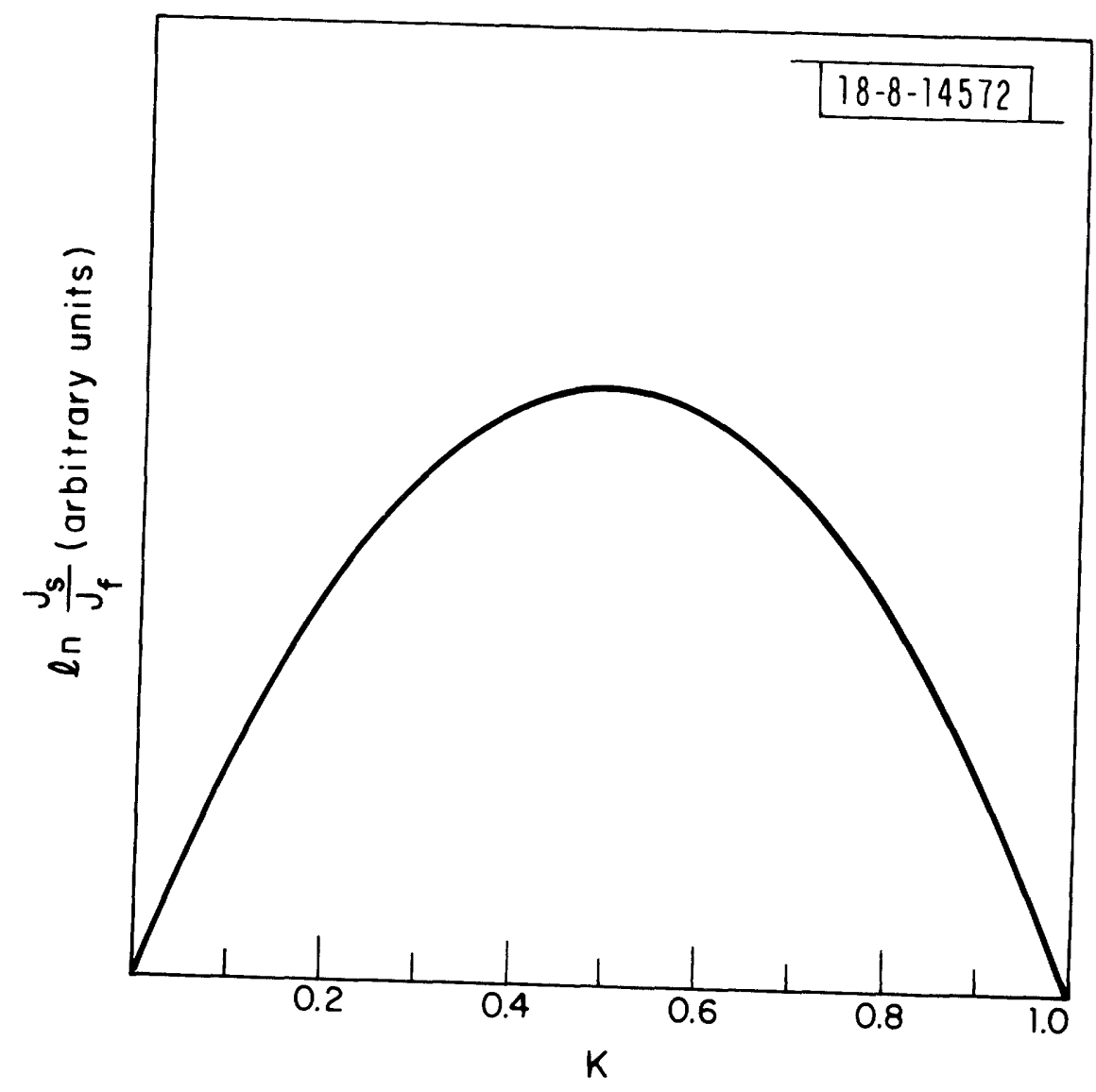

Fig. 5-8. A plot of the calculated ratio of nucleation rates at a step and on a plane, versus $k=\frac{d}{l}$ for a cubic crystalline deposit material. 
rate at a step site is always greater than the rate at a site on the flat plane. Another point is that the equilibrium nuclei orientation should be highly favored at a step because of its lower free energy. An example of this phenomenon for the case of a single crystal substrate appears in some work shown in Fig. 5-9 of G. Shinaoka and G. Komoriya for tin deposited on 76

steps in $\mathrm{NaCl}$. The tin islands which decorate the steps have a highly preferred orientation where $<001>$ is parallel to the cleavage steps and $\{100\}$ is parallel to the substrate.

In practice it has been difficult to test nucleation and decoration theories. This is because the critical nuclei sizes tend to be very small and are difficult to observe. In general deposited films which are claimed to be at the "nucleation" stage are actually well past nucleation and considerable coalescence may have already taken place. Some of the observed "decoration" effects may be the result of preferential growth at steps. 




Fig. 5-9. Transmission electron micrograph of tin nucleation on $\mathrm{NaCl}$. Note that the islands which lie along cleavage steps are preferentially oriented. (From work by Shinaoka and Komoriya) 76 


\subsubsection{ISLAND GROWTH}

As soon as nuclei form on a substrate the nucleation rate is significantly lowered in a region around each nucleus because of adatom depletion by the growing islands. Thus, following nucleation, a maximum density of nuclei is soon reached. Growth of the islands is then a diffusion controlled process where adatoms diffuse over the surface and are captured by stable islands. Under equilibrium conditions the islands, irrespective of their orientation at nucleation, should assume the equilibrium form predicted by the Gibbs-Wulff construction. This tends not to be the case during most deposition processes and the growing islands often continue to have a multiplicity of orientations at this stage. 


\subsubsection{COALESCENCE AND NETWORK FORMATION}

It is generally observed that the density of islands decreases monotonically with time from the maximum determined by the nucleation conditions. This is explained by the coalescence of growing islands illustrated in Fig. 5-10. When two islands touch, a neck or bridge if formed. This generally high curvature neck is rapidly el iminated by surface and volume diffusion until the two islands have coalesced to form a single island. As a general rule, two islands which have different orientations tend to recrystallize upon coalescence to yield a single crystal island. Such behavior has been observed to occur in times less than one second for islands as large as $100 \mathrm{~nm}$, for larger islands coalescence tends to be quite slow and reorientation is less likely. As a rule of thumb coalescence of islands less than $100 \mathrm{~nm}$ in size tends to result in a single island which tends to assume an equilibrium configuration. The dynamics of coalescence are not completely understood (as is very iittle of thin film growth), but its mechanism can probably be adequately described as a sintering process where the basic driving force is provided by the tendency of the island to minimize its surface free energy.

A convincing argument for the sintering model of coalescence is that it predicts the onset of the network stage of thin film growth (see Fig. $5-1$ ). According to the sintering model, the time required for coalescence of two islands increases approximately as the fourth power of the island radius. In effect this predicts a critical island size where coalescence will become slow compared to island growth. At this critical size coal- 


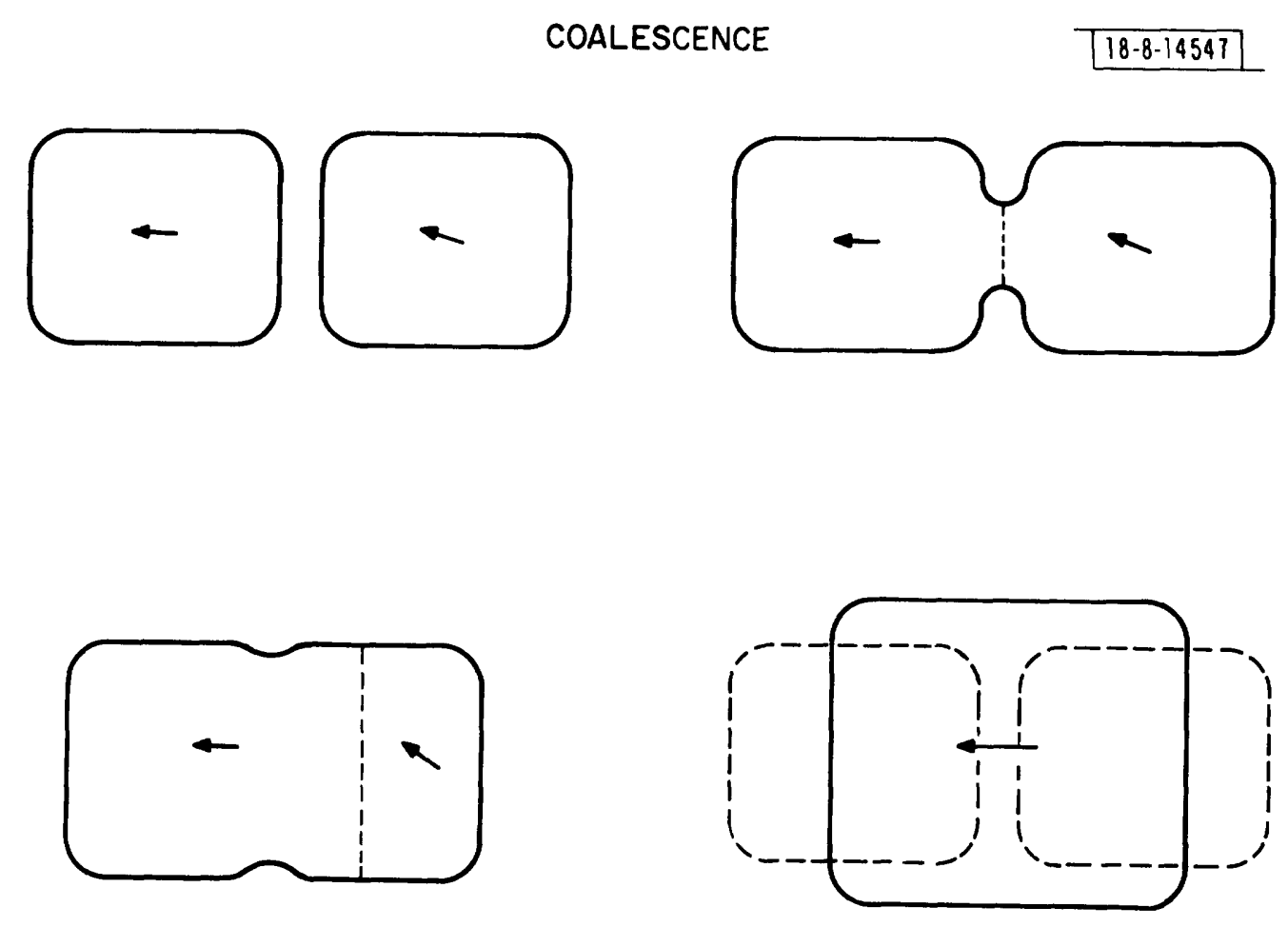

Fig. 5-10. The coalescence and recrystallization phenomenon for two islands is shown. 
escence of two islands is not completed before a third island contacts the compound island, etc. At this point a continuous network of interconnecting islands is rapidly formed and large scale reorientation essentially ceases. This phenomenon is illustrated in Fig. 5-11. If coalescence is rapid compared to growth a set of small randomly oriented islands can eventually form a large single crystal island as shown in Fig. 5-11(a). If coalescence is slow compared to growth the initial set of islands will form a continuous network of interconnecting grains as shown in Fig. 5-11(b). As growth continues the remaining channels and holes are filled by deposited material which tends to coalesce with the surrounding network structure and take on the local orientation of the network.

It is generally observed that single crystal deposits are not formed unless the individual islands have a single uniform orientation immediately prior to the network stage because of the difficulty of reorientation at that stage. 


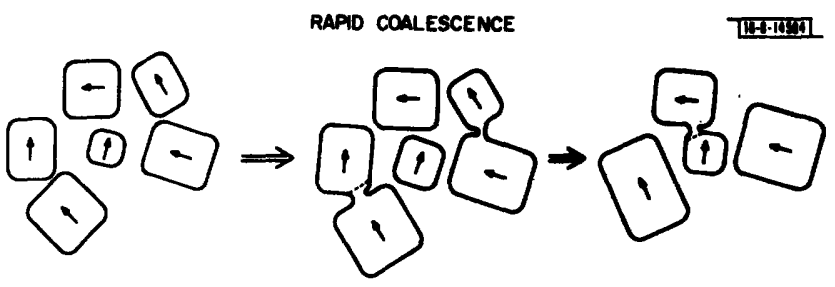

(a)
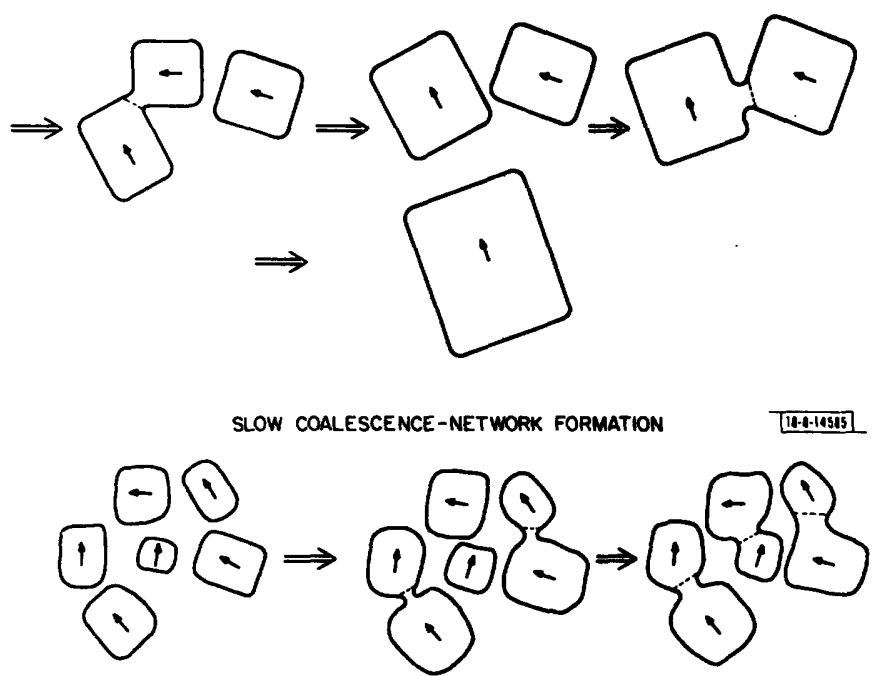

(b)

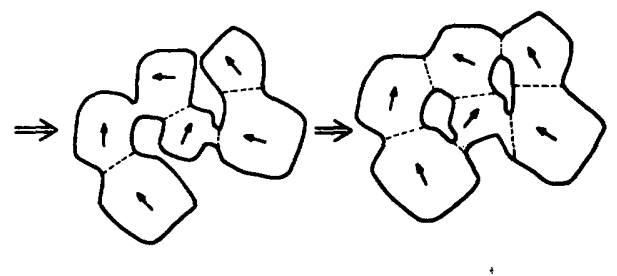

Fig. 5-11. a.) The coalescence of a group of growing islands is shown for the case of rapid coalesence and recrystallization. b.) The onset of the network stage of film growth is shown to occur when coalescence and recrystallization proceed slowly with respect to growth. 


\subsubsection{FIBER TEXTURES ON SMOOTH AMORPHOUS SUBSTRATES}

As mentioned in the introduction to this chapter the polycrystalline films which result from deposition of crystalline overlayers on smooth amorphous substrates are not necessarily entirely random. These films can be textured having some preferred crystallographic orientation. This phenomenon known as "fiber texture" has been observed for a great many substrate-deposit combinations. Bauer has reviewed the subject ${ }^{3}$ and has proposed a model to explain the observed textures. He finds that there are generally two kinds of textures; nucleation-equilibrium textures and growth textures. Nucleation-equilibrium textures appear to occur in systems which have a high adatom and island "mobility". Growth textures occur in systems with low mobility where the orientation of the deposit is determined by the direction of impinging atoms and the direction of fastest growth. The phenomenon of interest here is the nucleationequilibrium texture since its orientation is determined by the substratedeposit interfacial tensions.

The mechanism of formation of a nucleation-equilibrium textured film can be seen by considering the thin film growth of a crystalline overlayer material on a smooth amorphous substrate in light of the nucleation model given in sections 5.2.2 and 5.2.3.

From equation (5.10) and the arguments of section 5.2.2 one sees that under conditions of low supersaturation the minimum free energy nucleus orientation will be favored at the nucleation stage. These nuclei will have some particular crystallographic plane parallel to the substrate 
although they will have a random in plane "azimuthal" orientation. As growth continues the islands will coalesce as described in section 5.2.6 and can be expected to retain or acquire the minimum free energy configuration. Since there is no anisotropy in the plane of the smooth amorphous surface the individual single crystal islands will continue to have random orientations in the plane of the surface. When the network stage of film growth is reached, coalescence of the islands effectively ceases and because the islands had no preferred direction in the plane a polycrystalline film results. However, because the individual islands comprising the film had a preferred plane parallel to the plane of the substrate the final film has a preferred crystallographic direction normal to the substrate.

Bauer shows that this simple model correctly predicts the observed textures of most vapor deposited BCC, FCC, and tetragonal metals, and the alkali halides as deposited on smooth glass, amorphous $\mathrm{SiO}_{2}$, and amorphous carbon substrates. The model also predicts the observed textures of alkali halides deposited from aqueous solutions. 


\subsection{A MODEL FOR ORIENTATION OF THIN FILM DEPOSITS ON AMORPHOUS SUBSTRATES BY SQUARE-WAVE SURFACE-RELIEF STRUCTURES}

A detailed model of the influence on thin film growth of surfacerelief structures on amorphous substrates is presented in this section. General arguments given in Chapter II indicated that at equilibrium a crystalline overlayer should assume a uniform orientation relative to a suitable surface-relief structure on an amorphous substrate. The nonequilibrium process of thin film deposition was described in section 5.2 as a series of steps which can be understood qualitatively using familar thermodynamic concepts. We have seen in section 5.2.3 that the nucleation process can be dramatically influenced by the substrate surface-relief structure and in section 5.2.7 we have seen that a crystalline material deposited on a smooth amorphous substrate can have a fiber texture which is partially oriented. Here it is shown that a surface-relief structure on an amorphous substrate can have an influence at all stages of thin film growth and that this influence can result in the formation of a single crystal film on an amorphous substrate with the orientation predicted by the equilibrium model of section 2.4. 


\subsubsection{SURFACE RELIEF STRUCTURE CHARACTERIZATION}

The type of surface relief structure considered in this section is an array of vertical steps which is an idealization of the square-wave-grating structures which can be fabricated in amorphous $\mathrm{SiO}_{2}$ by the techniques described in Chapter III. The cross section of this idealized array of steps is shown in Fig. 5-12.

The step array will have a period, $s$, a height, $h$, a top width, $w_{t}$, and a groove width, $w_{g}$. The corners of the step will also have finite radii $R_{T}$ and $R_{G}$. The deposit material will be assumed to be cubic with interfacial tensions described by the polar plot of Fig. 2-1 (c). The arguments given here can of course be generalized to other materials and other surface-relief structures including the saw tooth structures shown in Figures $1-1$ and 2-2.

It is known, as detailed in section 5.2, that in order to obtain a single crystal film by the thin film growth process, the individual islands of a deposit should be uniformly oriented before the onset of the network stage of growth. Since it is expected that vertical steps as shown in Fig. 5-12 will directly influence only those islands which contact the step, there will be a maximum spacing of the steps determined by the island size at the network stage. For most materials and deposition conditions this requires that the step spacing be less than $200 \mathrm{~nm}$ (It is possible that this requirement could be relaxed if the network stage could be delayed by the use of a two dimensional grid type structure such as shown in Fig. 5-13). 


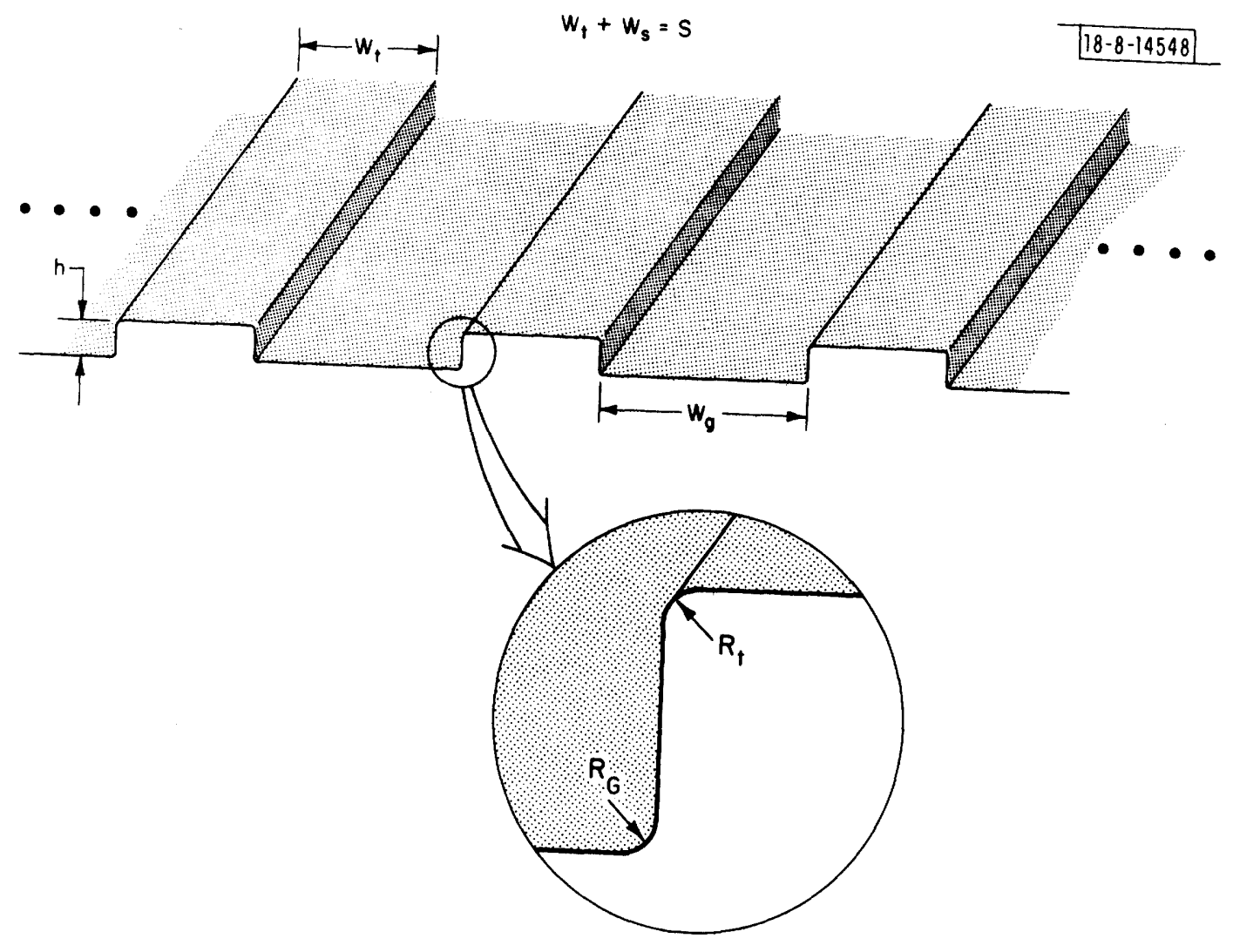

Fig. 5-12. An idealized representation is shown of the square-wave surface-relief structure used for overlayer orientation experiments. The structure consists of a periodic array of vertical steps of height, $h$, period, $s$, top width, $w_{t}$, and groove width, $w_{g}$. The edges have some finite radii of curvature $\mathrm{R}_{t}$ and $\mathrm{R}_{\mathrm{g}}$. 


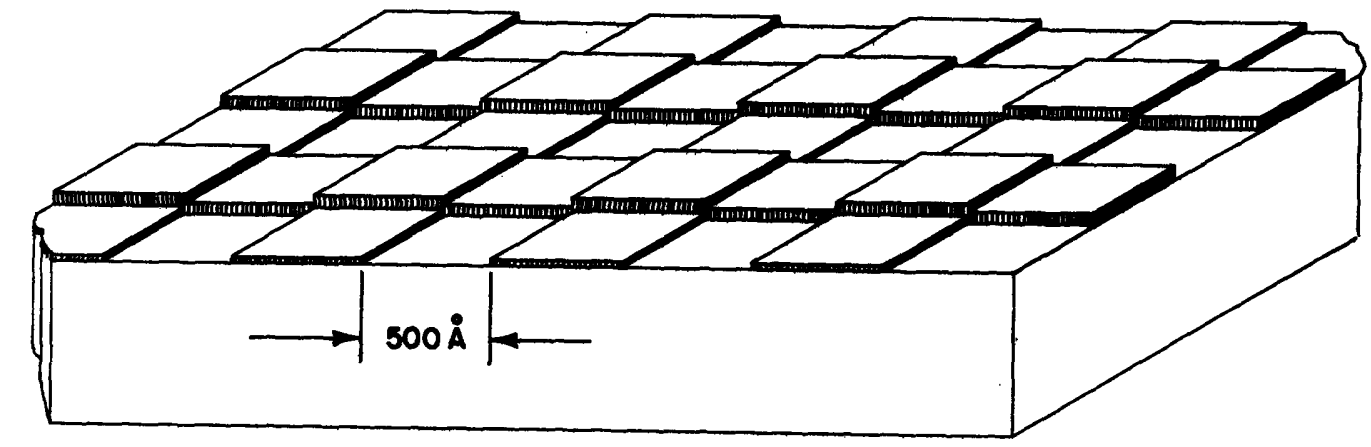

Fig. 5-13. A schematic representation of a grid-type square-wave structure which could be used to delay the onset of the network stage of film growth and induce overlayer orientation. 
Conceptually one would also want to minimize the top width, $w_{t}$, since islands in that region would not contact steps (once again the more complex structure of Fig. 5-13 would reduce this problem). However, for the first experiments, minimization of the top width is not important and perhaps not desirable since unexpected orientation effects might very well occur on the grating tops.

The optimum height of the steps is expected to be determined by several factors. As indicated in section 2.3 the anisotropy in the plane of the interfacial tension increases with the step height. Thus, there will probably be some minimum step height required to induce orientation in any particular case. The smoothness and possibly the dislocation density of the final film will also depend upon the step height. For these reasons the height should be minimized. Because the deposit on the top of the grating is likely to experience a strong orientational effect only after it has coalesced with the deposit in the groove, the height should also be less than the average thickness of the islands at the network stage.

The radii of curvature $R_{g}$ and $R_{t}$ at the groove corners should be minimized to enhance decoration and alignment effects. $\left(\mathrm{SiO}_{2}\right.$ structures fabricated by the techniques of Chapter III have $\mathrm{Rg}$ and $\mathrm{R}_{t}$ estimated to be less than $5 \mathrm{~nm})$. 


\subsubsection{NUCLEATION AND GROWTH ON A SQUARE WAVE GRATING ON AN AMORPHOUS SUBSTRATE}

If the radius, $\mathrm{Rg}$, is considerably less than the radius, $r^{*}$, of the critical nucleus of the deposit and the deposit adheres to the substrate, the corners of the grooves should be decorated at the nucleation stage by the deposit. This is evident from . Fig. 5-8 of section 5.2.4. To insure that this occurs the deposition should begin under conditions of low supersaturation. The sequence of growth on the surface-relief structure should depend critically upon the "strength" of the decoration of the steps and the size of the islands at the network stage. Several deposition senarios are illustrated in Figures 5-14, 5-15, and 5-16. In these illustrations a schematic top view is shown of hypothetical growth sequences from nucleation to network formation for a cubic deposit material on a square-wave structure.

If the steps are sufficiently close together and decoration is strong it is possible that all islands could be confined to the steps at the nucleation stage as shown in Fig. 5-14. (It is also possible that nearly all of the idlands coild be confined to the steps at an early stage if that configuration is energetically favorable and the small islands are mobile such that they could migrate to the steps irrespective of their position at nucleation). The confinement of all growing islands to the steps is probably an optimum situation, since it insures that the growing islands would continuously experience the orienting influence of the steps. 


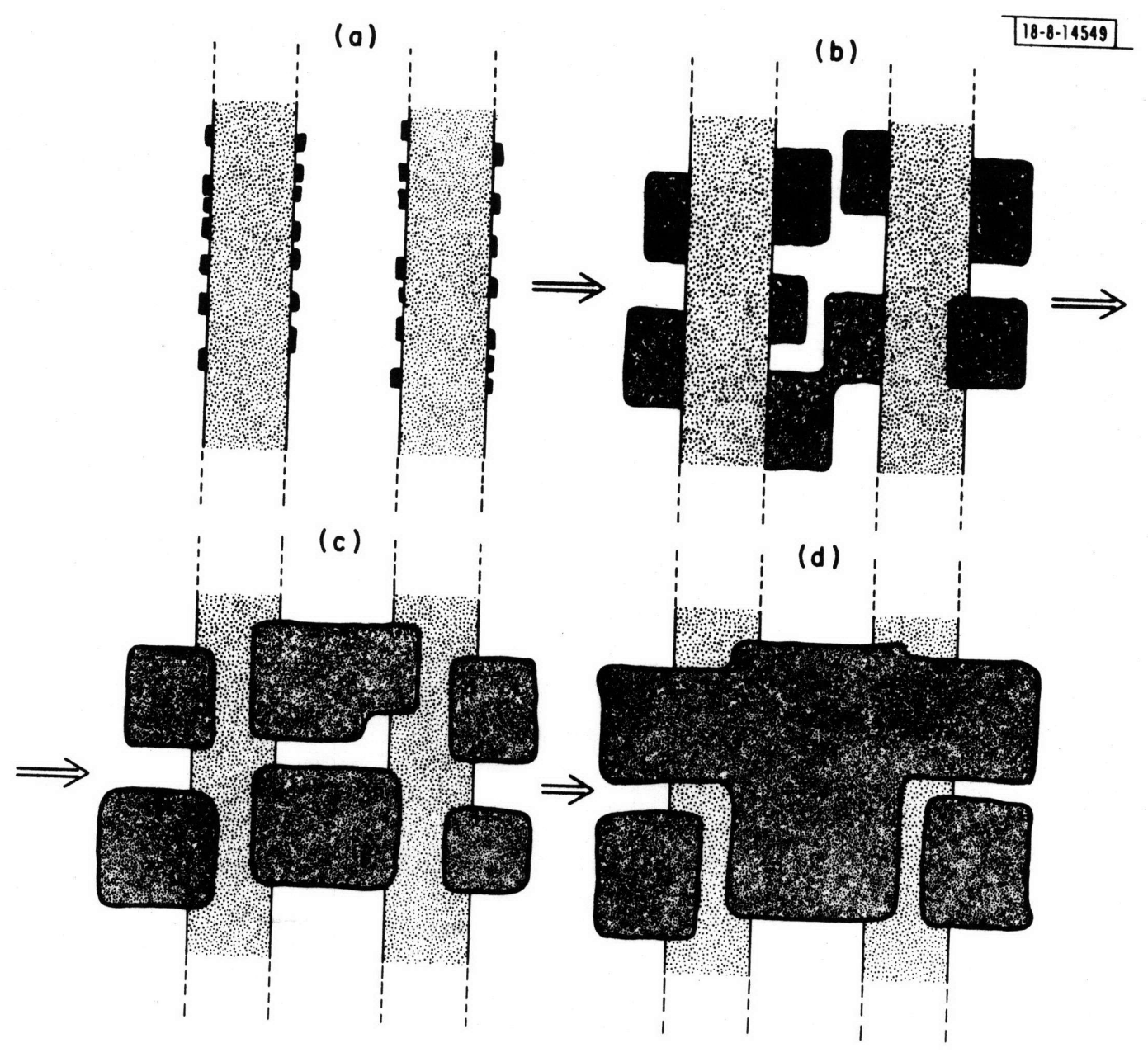

Fig. 5-14. The sequence of growth for a cubic material on a squarewave structure for the case of perfect "decoration" of the vertical steps. 


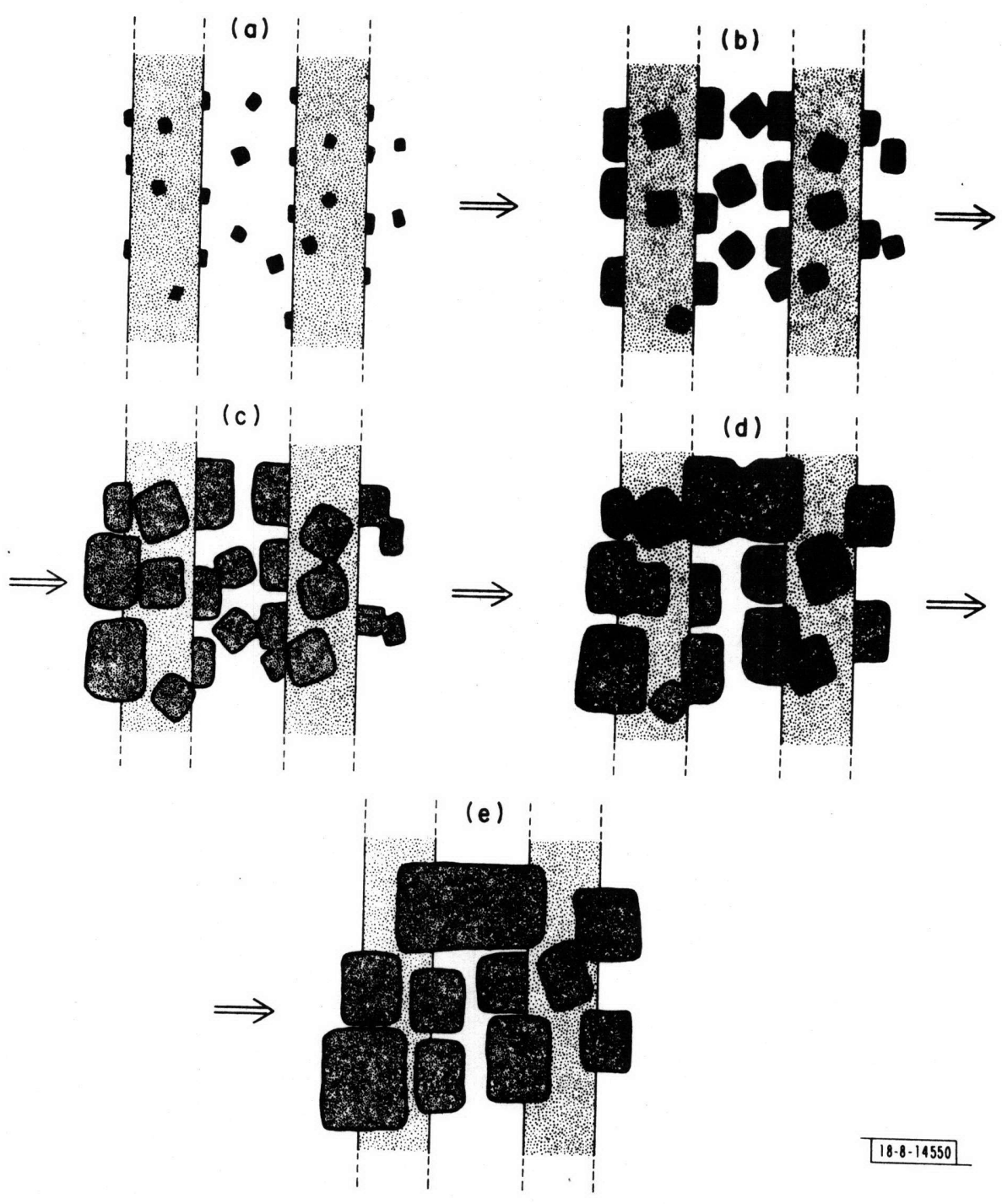

Fig. 5-15. The sequence of growth of a cubic material on a square-wave structure where nuclei occur both at the steps and between the steps. 


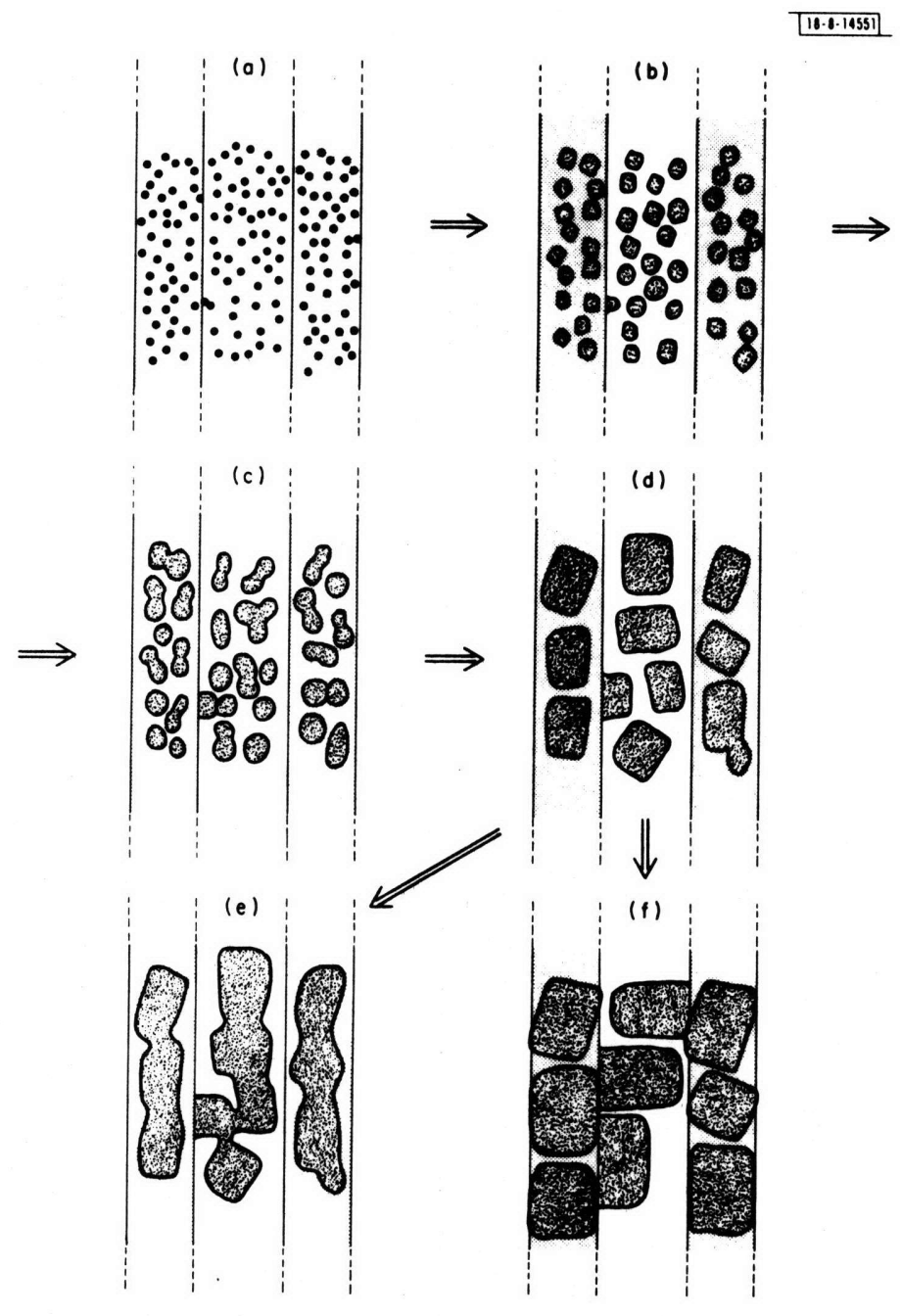

Fig. 5-16. The sequence of growth of a cubic material on a square wave structure where the nucleation is random and few nuclei occur at the steps. If the network stage of thin film growth occurs before the islands contact the steps, as in $(e)$, oriented growth would be unlikely. 
As shown in Fig. 5-6 the minimum free energy equilibrium orientation of a cubic island at a step with the assumed interfacial tensions would be with $\{100\}$ paralle $\}$ to both the substrate plane and the step wall. In addition it is easily seen that if the deposit adheres to the substrate the free energy of an island of constant volume with the equilibrium orientation would be smaller at the step than on the flat substrate. Thus, as explained in section 5.2.4, under conditions of low supersaturation and small $\mathrm{Rg}$ (as compared to the critical nuclei size) a cubic deposit should preferrentially nucleate and grow at the steps with the equilibrium orienation. Under these ideal conditions the oriented deposit would nucleate and grow from the steps to fill the grating grooves with single crystal material, which would then grow to cover the top of the grooves to yield a uniform single crystal film.

If decoration of the steps was not perfect and islands also nucleated in the groove area between the steps or on the grating tops as shown in Fig. 5-15(a) the mobility of the islands would play an important role in determining the growth sequence. If the nuclei were highily mobile they could migrate to the steps and would tend to become fixed at the steps because the step location is energetically favored. If essentially all of the nuclei were confined to the steps in this way, growth would proceed in the way shown in Fig. 5-14. If the islands were not mobile growth would proceed until islands contacting the steps coalesced with the unconfined islands at which time the compound islands should take on the minimum free energy configuration in contact with the step. This sequence 
is shown in Fig. 5-15. As the oriented islands in the grooves grew. above the steps they would eventually contact the unoriented islands on the top of the grating. Upon coalescence these islands should also assume the minimum free energy orientation and a single crystal film would result. If nucleation was random, with few nuclei occuring at the steps and the islands were not mobile, the orientation effect would be considerably reduced. This situation is illustrated in Fig. 5-16. As nuclei grew and coalesced, they would tend to recede from the step. The reason for this is evident from Fig. 5-10. The position of the center of the island resulting from the coalesence of two islands tends to be at the "center of gravity" of the two parent islands. Thus, an island which was originally near a step but not contacting a step, would tend to move away from the step because of the unidirectional population of islands. Eventually the islands would fill the region between the steps and contact the vertical walls of the step. If this occurred at an island size such that reorientation was still possible the island could then assume the oriented equilibrium configuration as shown in Fig. 5-16 (f). The oriented islands in the grooves would then grow to contact the islands on the top of the gratings, etc. If growth should proceed as in Fig. 5-16 (a)-(d), but the islands did not contact the steps before the network stage was encountered in the grating grooves, the emergence of an oriented film would be unlikely since the randomly oriented islands between the steps and on top of the grating would be effectively locked in their random orientations before they contacted the steps. This situation is illustrated in Fig. 5-16(é). 
It is of course possible that a randomly oriented film resulting from the above senario could be annealed after deposition to yield an oriented equilibrium configuration. In fact there is considerable experimental evidence that randomly oriented continuous polycrystalline films of several materials including $A 7$, , tin, and silicon, on amorphous substrates can undergo considerable reorientation and acquire strong fiber textures during annealing by heating. It is also known that water soluble films such as alkali halides can be annealed by placement in humid atmospheres. 
5.4 DEPOSITION OF CRYSTALLINE OVERLAYERS ON SQUARE-WAVE GRATINGS IN AMORPHOUS $\mathrm{SiO}_{2}$-EXPERIMENTS

\subsubsection{EXPERIMENTAL PROCEDURE}

The experimental procedure used to investigate the deposition of thin solid films on square-wave surface relief structures is outlined in Fig. 5-17. The surface-relief structure is fabricated in amorphous $\mathrm{SiO}_{2}$ on a silicon substrate using the methods described in Chapter III. The overlayer material is then deposited on the surface-relief structure. At this point the crystallinity of the overlayer can be examined using reflection high energy electron diffraction (RHEED). Alternatively, the sample can be thinned by anisotropically etching the silicon away. After thinning, the deposit and surface-relief structure can be observed in a transmission electron microscope. At the same time selected area electron diffraction can be performed to determine the crystallinity of the deposit. (Sometimes for convenience the substrate is thinned before deposition.) 


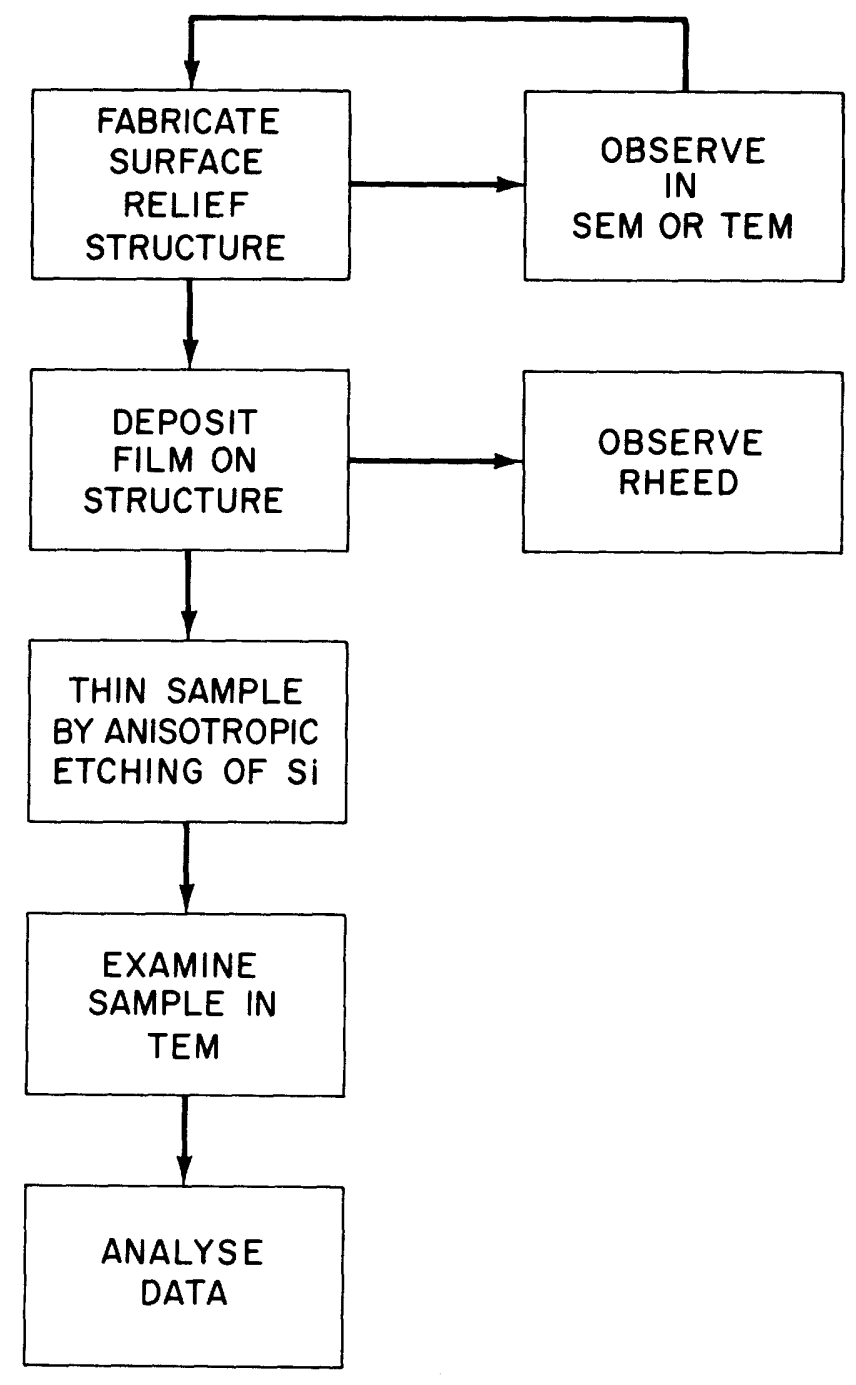

Fig. 5-17. The procedure used for investigating the effect of surface relief oratings on thin film growth. 


\subsubsection{DECORATION EXPERIMENTS}

The proposed optimum growth sequence on a square-wave structure described in section 5.3.2 and illustrated in Fig. 5-14 relies upon strong decoration of the grating steps at the nucleation stage. Because of the perceived importance of decoration in determining the growth sequence a study of the decoration effect was conducted. From the classical thermodynamic analysis of nucleation-decoration presented in section 5.2.4 it is seen that a critical parameter which determines whether decoration will occur is the curvature, $\mathrm{Rg}$, of the grating groove corner as compared to the size of the critical nucleus. If the radius of curvature, $\mathrm{R}_{\mathrm{g}}$, is smaller than the radius of the critical nucleus decoration should occur. If, $\mathrm{Rg}$, is larger than the critical nucleus decoration is expected to be very weak or nonexistant. The critical nuclei for materials deposited by evaporation or sputtering in high vacuum are believed to be smaller than $1 \mathrm{~nm}$. At the present the resolution of direct methods for viewing surface-relief structures is inadequate to determine accurately the radi $i$ of curvature $R_{t}$ and $R_{g}$. TEM and SEM observations indicate that an upper limit on $\mathrm{Rg}$ for the best structures is $\approx 5 \mathrm{~nm}$.

To determine whether decoration would occur on an array of steps fabricated by the techniques of Chapter III several experiments were performed. The first decoration experiments were done using gold because of the ease of observation of small islands in TEM due to the relatively large scattering of electrons by gold. Fiber textures with a. $\langle 111\rangle$ direction normal to a smooth $\mathrm{SiO}_{2}$ substrate are observed for. 
gold, thus the minimum interfacial tension of gold with $\mathrm{SiO}_{2}$ occurs for $\{111\}$ parallel to the interface. However, the anisotropy of the interfacial tension of gold is sma11. In fact, the decoration phenomenon for gold can probably be accurately modeled by assuming an isotropic interfacial tension. Using 1 published values of interfacial tension for gold and $\mathrm{SiO}_{2}$ the contact angle of gold on $\mathrm{SiO}_{2}$ is estimated to be $90^{\circ}$. Fig. 5-7 indicates that decoration should be very strong (assuming $\mathrm{Rg}$ is sufficiently smal1). Decoration experiments were done on square-wave $\mathrm{SiO}_{2}$ structures fabricated by three different techniques. The procedures used are outlined in Fig. 5-18. Details of the techniques are given in Chapter III. Briefly, a $320 \mathrm{~nm}$ period grating was exposed in PMMA using $\mathrm{Cu}_{L} X$-ray lithography. The PMMA was then used directly as an etching mask during ion-beam etching or was used to define a grating structure in another material by the liftoff process. A square-wave grating structure in amorphous $\mathrm{SiO}_{2}$ was produced by liftoff of evaporated $\mathrm{SiO}_{2}$. (Actually the evaporated $\mathrm{SiO}_{2}$ is not stochiometric and is designated $\mathrm{SiO}_{\mathrm{X}}$.) The third fabrication method also used the liftoff process. A $10 \mathrm{~nm}$ thick film of chromium was evaporated over the PMMA structure and lifted off to yield a chromium grating on the $\mathrm{SiO}_{2}$ substrate which in turn was used as an etching mask during reactive ion etching of the $\mathrm{SiO}_{2}$. Following reactive-ion-etching the chromium was removed using an aqueous chemical etch. These three different structures will be referred to as the ion-beam etched, liftoff, and reactive-ion-etched structures.

Because of the redeposition effects discussed in section 3.4.1 the ion 
PREPARE SUBSTRATE MATERIAL

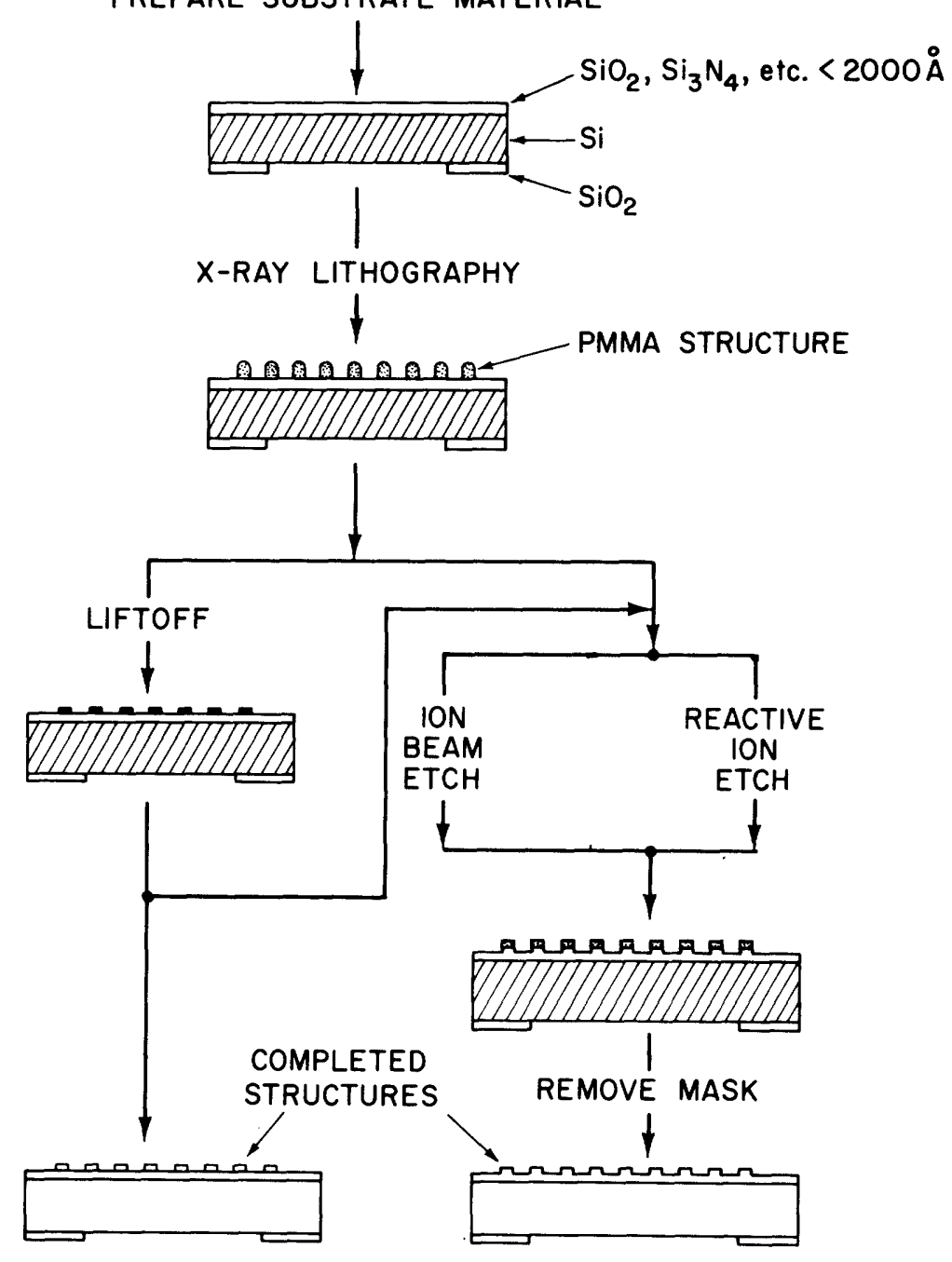

Fia. 5-18. The procedure used for fabricating square-wave surface relief structures. 
beam etched structure probably had the largest $\mathrm{Rg}$. The profiles of the liftoff and reactive-ion-etched structures more closely approached the ideal square-wave structure and $\mathrm{Rg}$ for these structures was probably somewhat smaller than the $\mathrm{Rg}$ of the ion-beam-etched structure.

Gold was deposited on the structures by ion-beam sputtering. The substrates were heated to $\approx 300{ }^{\circ} \mathrm{C}$. The deposition rate was $\approx 0.1 \mathrm{~nm} / \mathrm{sec}$. An average thickness of $\approx 1.5 \mathrm{~nm}$ of gold was deposited. (The deposition parameters closely approximate those used by Bassett for the decoration of cleavage steps on $\mathrm{NaCl}$ shown in Fig. 5-2). Following deposition the substrate was allowed to cool slowly to room temperature. The substrate was then thinned by anisotropically etching the silicon using the methods described in section 3.5 and Appendix A. The deposit was examined in a TEM. Micrographs of deposits on the ion-beam etched, liftoff, and reactiveion-etched structures are shown in Figures 5-19, 5-20, and 5-21 respectively. The size and shape of the islands indicates that some coalescence has already occurred. None of the structures exhibit strong decoration. The islands appear to have avoided the steps in all of the Figures. . This is probably explained by the coalescence effect illustrated in Fig. 5-16 where nucleation is random and coalescence tends to make the islands migrate toward their center of gravity and hence away from the steps. The larger island size and more pronounced avoidance of the steps in the case of the reactive-ion-etched structure of Fig. 5-21 is explained by the fact that this deposition was done at a substrate temperature of $500{ }^{\circ} \mathrm{C}$ versus $300{ }^{\circ} \mathrm{C}$ for the other structures. The lower density of islands in the 


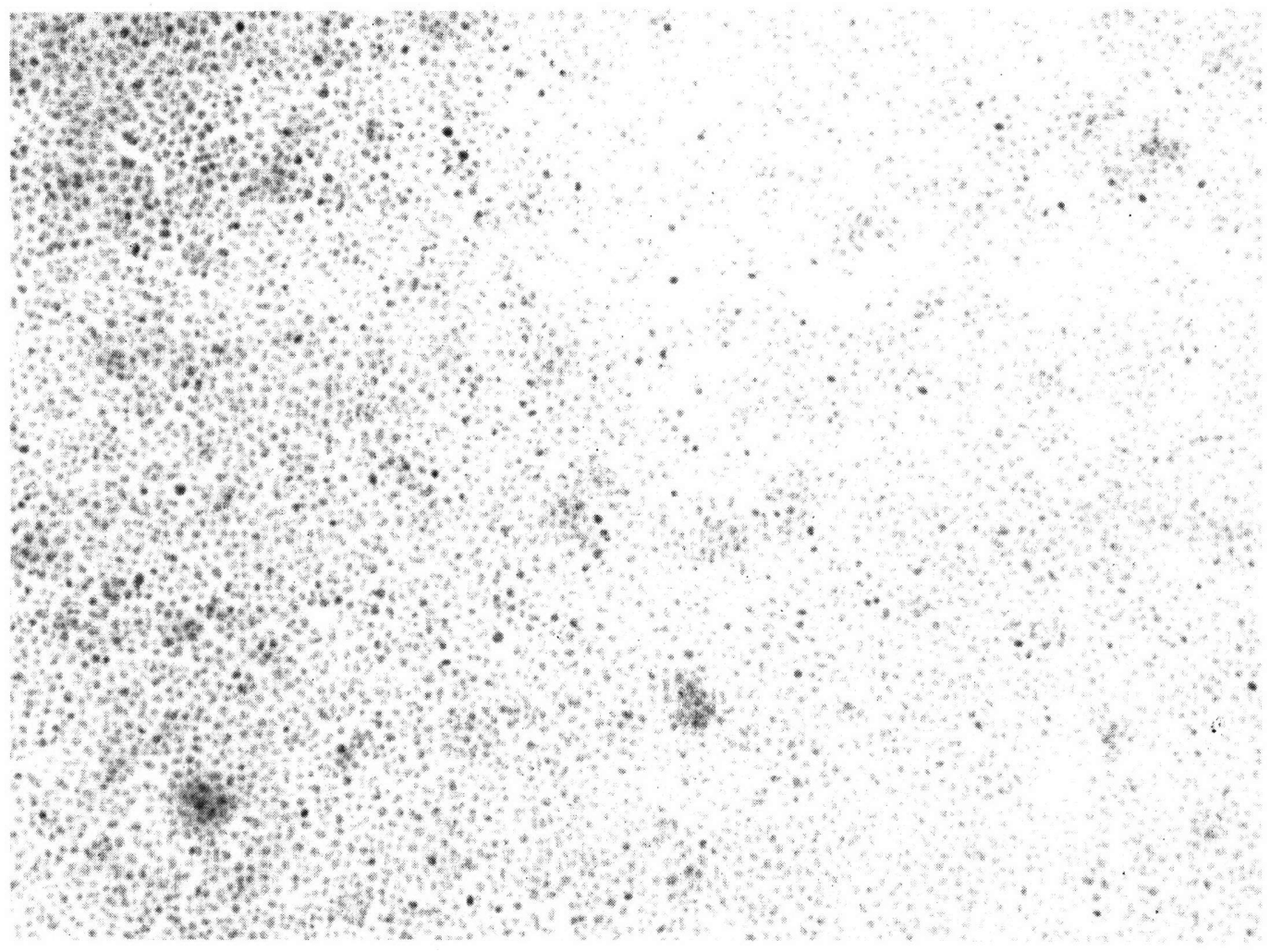

Fig. 5-19. Transmission electron micrograph of gold islands on an amorphous $\mathrm{SiO}_{2}$ surface with a $1.5 \mathrm{~nm}$ deep, $320 \mathrm{~nm}$ period, square-wave surface-relief grating fabricated using ion-beam etching. The magnification is $\approx 100 \mathrm{KX}$. 


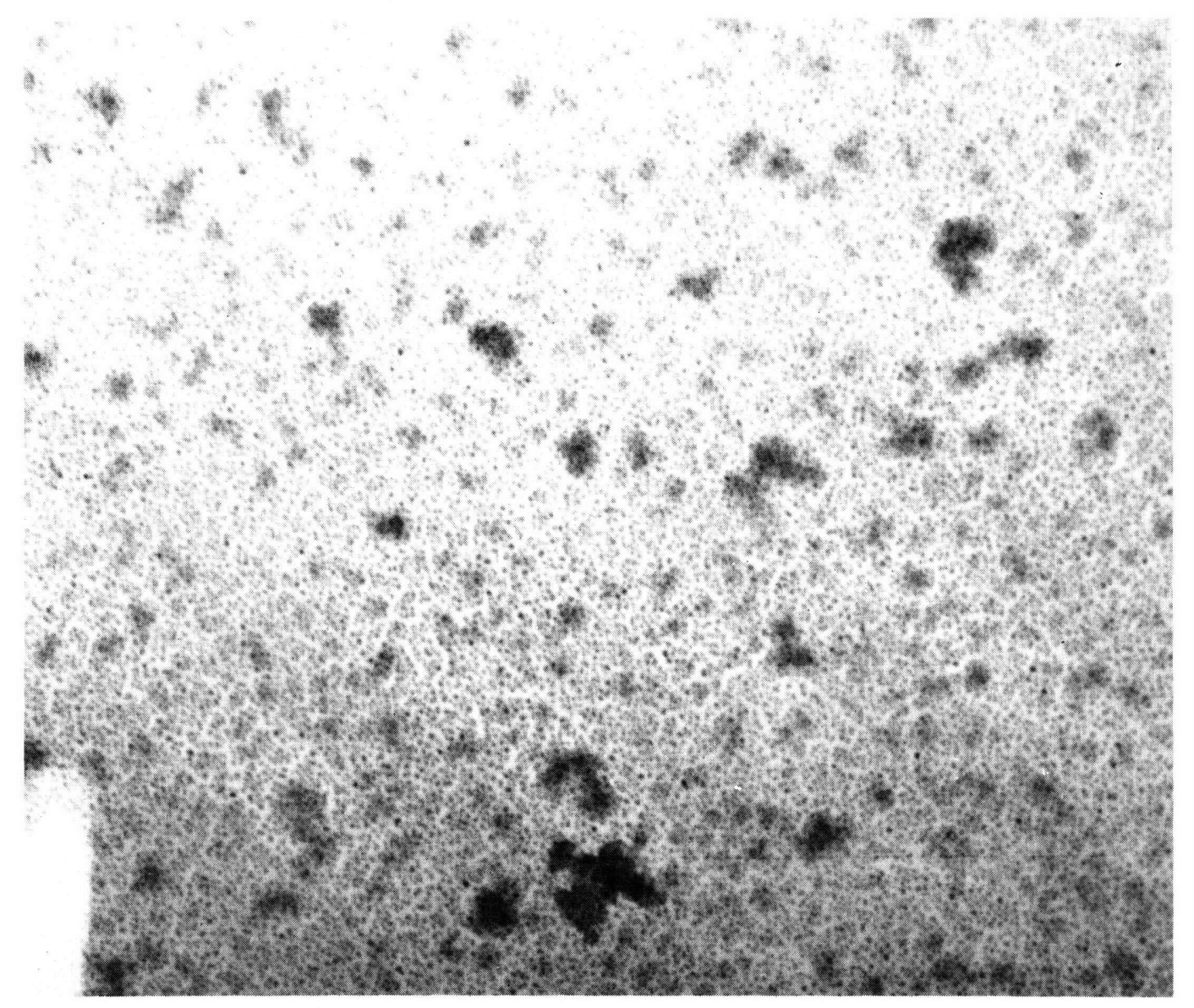

Fig. 5-20. Transmission electron micrograph of a gold deposit $1.5 \mathrm{~nm}$ average thickness on a square-wave grating surface-relief structure in $\mathrm{SiO}_{2}$ fabricated by liftoff of evaporated $\mathrm{SiO}_{2}$. The grating depth is $\approx 10 \mathrm{~nm}$ and the period is $320 \mathrm{~nm}$. The magnification is $\approx 62 \mathrm{KX}$. 


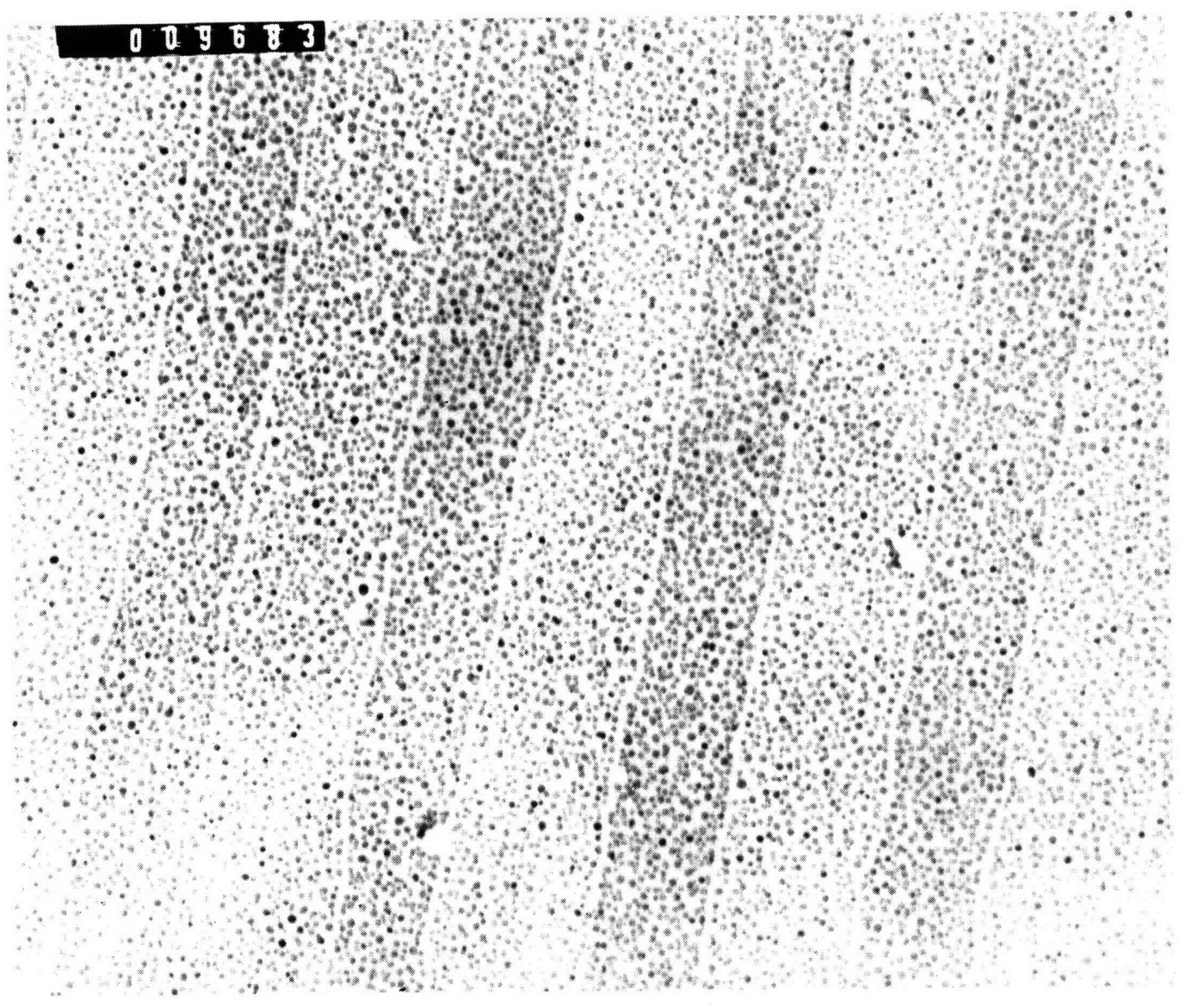

Fig. 5-21. Transmission electron micrograph of a gold deposit on a $5 \mathrm{~nm}$ deep, $320 \mathrm{~nm}$ period square-wave grating in amorphous $\mathrm{SiO}_{2}$ fabricated using reactive-ion etching. The average gold thickness is $\approx 1.5 \mathrm{~nm}$. The magnification is $\approx 115 \mathrm{KX}$. 
grooves versus the grating tops in Fig. $5-21$ is probably explained by contamination in the grooves. Possibly a layer of carbon was deposited in the grooves during reactive-ion-etching. If carbon was present the lower density of nuclei would be consistent with gold deposition experiments done on carbon strips defined on an $\mathrm{SiO}_{2}$ substrate by liftoff. A micrograph of the results of this experiment is shown in Fig. 5-22 which shows a much lower nuclei density on the carbon strips.

A slightly higher density of islands in the grooves at or near the steps can be discerned in some areas of the micrographs particularly for the liftoff structure of Fig. 5-20. This may be a weak decoration effect. The lack of strong decoration is probably due to the small critical nucleus size as compared to the radius of the corners of the $\mathrm{SiO}_{2}$ structures.

The fact that strong decoration does not occur for gold is disappointing because it is believed that the growth scenario of Fig. 5-14, where oriented growth proceeds from the steps, cannot be realized for gold and other materials that nucleate in the same fashion. Nevertheless, as shown in section 5.3.2 and Figures 5-15 and 5-16, orientation could still occur during the island growth and coalescence stage even in the absence of decoration.

Further deposition experiments of gold on square-wave structures were not done because the well known $\{111\}$ fiber texture of gold on $\mathrm{SiO}_{2}$ is not directly compatible with the square-wave structure since the angles between $\{111\}$ planes do not equal 90 degrees, and strong alignment effects are not expected. 


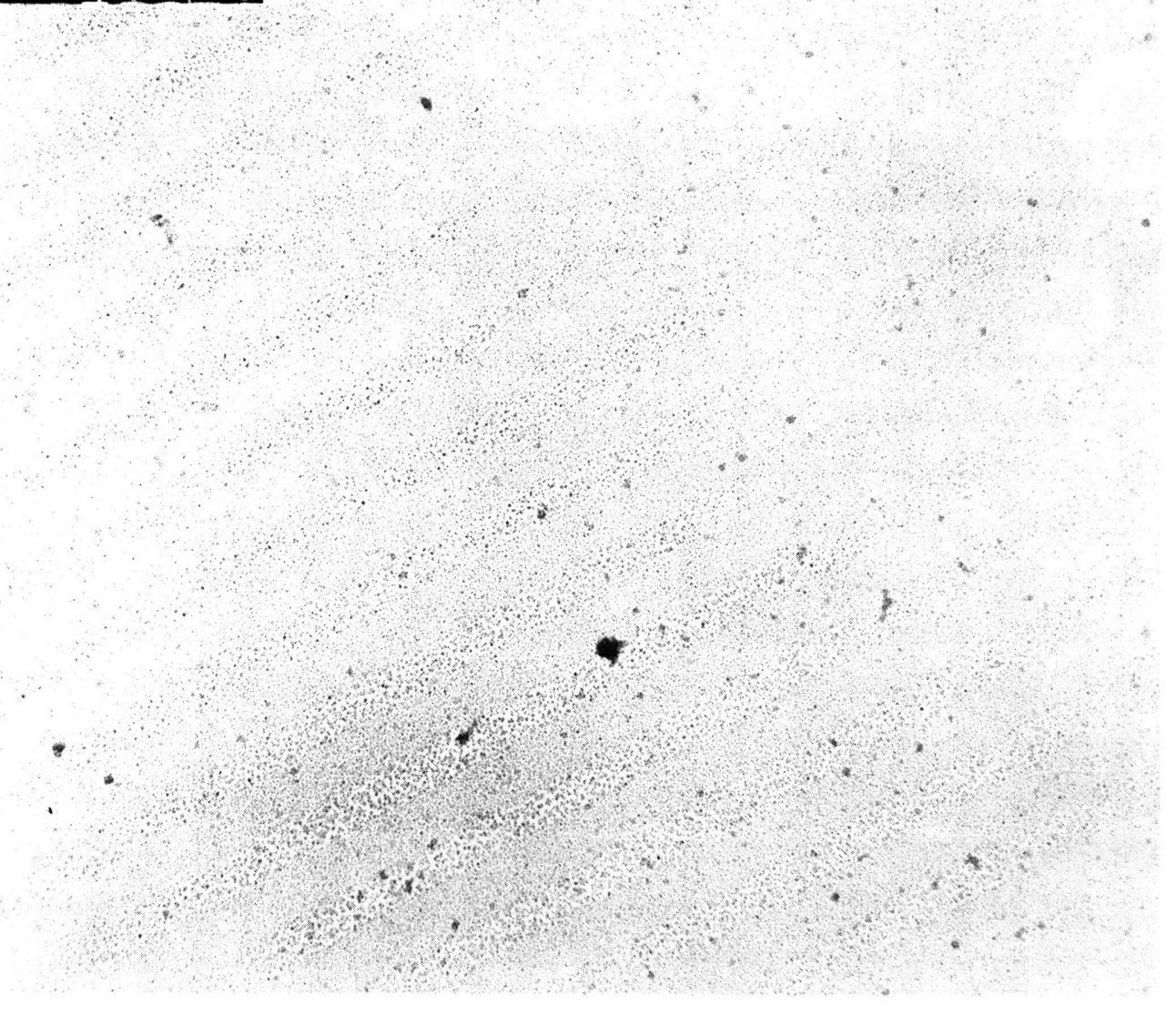

Fig. 5-22. Transmission electron micrograph of a gold deposit of $1.5 \mathrm{~nm}$ average thickness on an $\mathrm{SiO}_{2}$ substrate on which a $320 \mathrm{~nm}$ period grating of $10 \mathrm{~nm}$ thick carbon strips has been defined by the liftoff process. The lower island density occurs on the carbon strips. The magnification is $\approx 28 \mathrm{KX}$. 


\subsubsection{POTASSIUM CHLORIDE DEPOSITION EXPERIMENTS}

The alkali halides deposited by evaporation in high vacuum or from an aqueous solution are known to exhibit strong $\{100\}$ fiber structures on smooth amorphous materials. Deposited on a square-wave grating structure on $\mathrm{SiO}_{2}$ they should orient with' $\{100\}$ parallel to the substrate and <100> parallel to the groove direction.

Deposition from solution is convenient because the deposit can be applied, examined, and removed without damaging the substrate. Several deposition experiments can be performed on the same structure. Unfortunately deposition from solution is difficult to control. If small deposit islands are to be obtained relatively high supersaturations must be achieved during deposition. These high supersaturations are obtained by rapidly evaporating the water from a thin layer of saturated solution placed on the surfacerelief structure. This process is by its nature uncontrolled. However, with this deposition process small deposit islands can be obtained consistently. Deposition by thermal evaporation in high vacuum can be controlled with great precision. Deposition times and rates are easily measured. However, the mobility of the deposit is much smaller during vacuum evaporation than during deposition from aqueous solution. A practical difficulty associated with vacuum evaporation deposition of $\mathrm{KCl}$ is that $\mathrm{KCl}$ is hydroscopic and its presence in a vacuum system can damage it an degrade the vacuum performance. For these reasons all depositions of $\mathrm{KCl}$ were done from aqueous solutions. Tin has a higher mobility than $\mathrm{KCl}$ and is more 
compatible with high vacuum practices. It was used for thermal-evaporation-invacuum deposition experiments. Tin deposition experiments are discussed in section 5.4.4.

The surface-relief structures used for $\mathrm{KCl}$ depositions were fabricated using reactive-ion-etching. The $\mathrm{SiO}_{2}$ substrates used were films $100 \mathrm{~nm}$ thick over a $100 \mathrm{~nm}$ thick amorphous $\mathrm{Si}_{3} \mathrm{~N}_{4} \mathrm{film}$. Both films were grown on $a^{\prime}\{100\}$ oriented silicon wafer by a commercial chemical vapor deposition process. As illustrated in Fig. 5-18 and detailed in Chapter III, a grating was exposed in PMMA using $\mathrm{Cu}_{L} X_{\text {-ray }}$ lithography, followed by liftoff of $10 \mathrm{~nm}$ of chromium, followed by reactive-ion etching of the $\mathrm{SiO}_{2}$ to a depth of $25 \mathrm{~nm}$ in $\mathrm{CHF}_{3}$ gas. The chromium was removed with an aqueous chromium etch and the substrate was thoroughly rinsed in water. $10 \mathrm{~nm}$ of chromium and $100 \mathrm{~nm}$ of gold were then evaporated over the entire grating area to act as a protective covering during anisotropic etching of the substrate. The silicon underlying the $\mathrm{Si}_{3} \mathrm{~N}_{4}$ and $\mathrm{SiO}_{2}$ films was etched away using ethylene diamine pryrocator.hol (See Appendix A). After etching of the silicon, the gold and chromium were removed by chemical etching. Because the substrates were now only $200 \mathrm{~nm}$ thick they could be viewed by transmission electron microscopy using both conventional TEM and STEM systems.

The $\mathrm{KCl}$ was deposited by flooding the $\mathrm{SiO}_{2}$ grating with a solution of $\mathrm{KCl}$ in water and then gently blowing nitrogen gas over it to promote evaporation, supersaturation, and crystal growth. (Experiments indicate that crystal orientation in the grating area was uncorrelated with the blowing direction.) Prior to storage in a desicator, while waiting for observation in the STEM, the sample is unavoidably exposed to a humid atmosphere for a few minutes. This may very well result in some annealing of the deposit. 
Figure $5-23$ is a scanning-transmission-electron-micrograph of a $\mathrm{KCl}$ deposition. The sides of nearly all of the rectangular $\mathrm{KCl}$ islands are aligned parallel and perpendicular to the surface grating. Virtually all of the small islands are located in the grating grooves (the lighter stripes). Selected area electron diffraction patterns, taken in a scanning transmission electron microscope (STEM), confirm that the' $\{100\}$ npilanes of the crystallites are parallel to the substrate surface and the $\langle 100\rangle$ directions are normal to the flat sides of the crystallites. Outside the grating area the $\langle 100\rangle$ directions of the crystallites showed no preferred orientation in the plane of the substrate. (An optical micrograph of a $\mathrm{KCl}$ deposit on a flat $\mathrm{SiO}_{2}$ substrate is shown in Fig. 5-24). A series of (TEM) micrographs of different areas of $\mathrm{KCl}$ deposit on the same sample are shown in Fig. 5-25. A histogram of the measured deviation of the $400>$ direction of the crystallites from the grating groove direction is shown in Fig. 5-26. The equivalent random distribution is also plotted in the Figure.

Because the smallest islands observed in the micrographs are located at the steps and nearly all of the islands are located in the grating grooves it appears that the steps may have been"decorated", and oriented growth has proceeded from the steps. Because of the relatively low supersaturations which are achieved during deposition from aqueous solution the critical radius of the $\mathrm{KCl}$ nucleus is expected to be larger than the critical radii for depositions by thermal evaporation in vacuum. In fact it is believed that the size of the critical nucleus under these low supersaturation conditions could be larger than the $5 \mathrm{~nm}$ (maximum) radius of the 


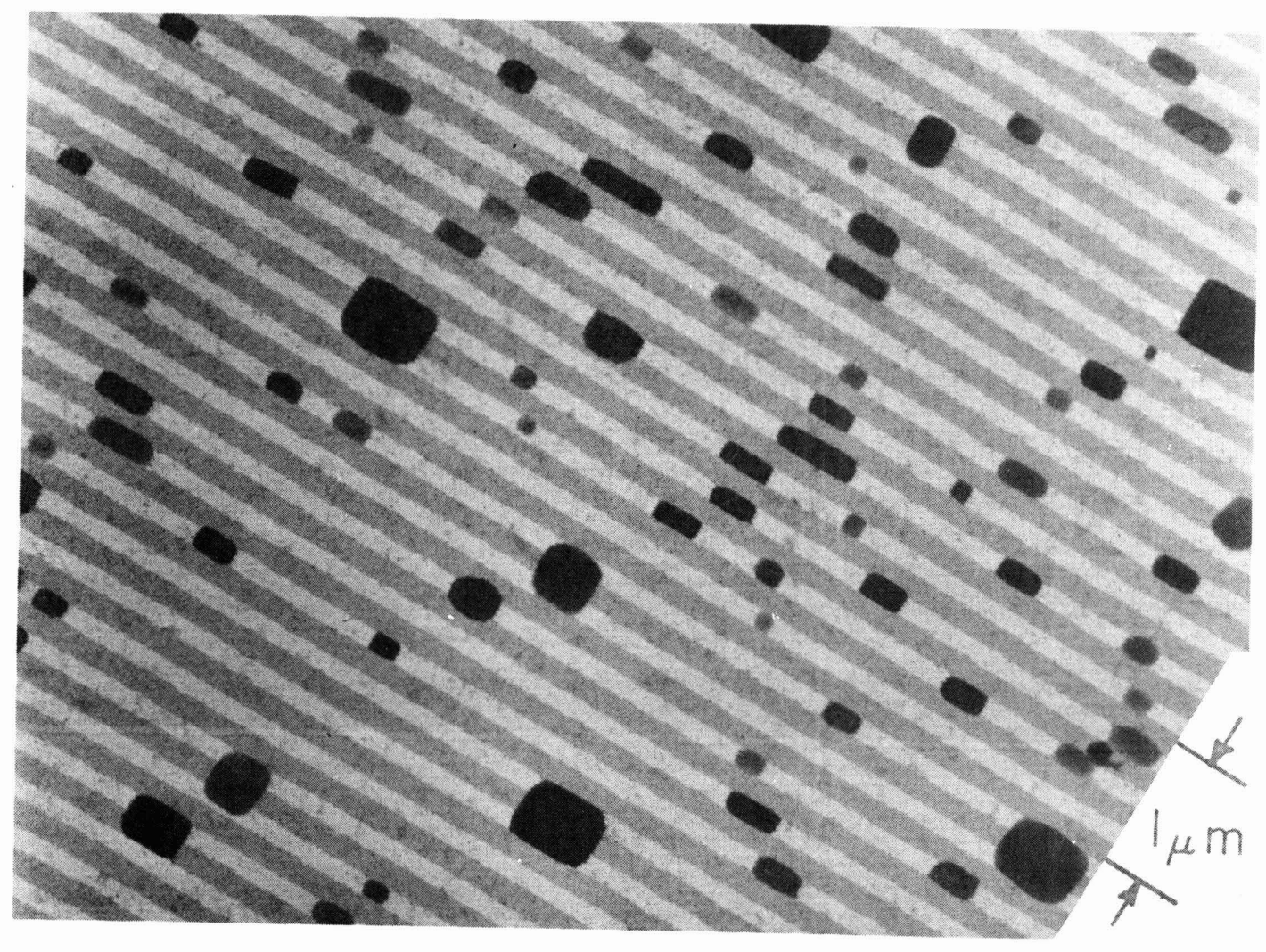

Fig. 5-23. Scanning-transmission-electron micrograph of KCl crystallites grown on a $320 \mathrm{~nm}$ spatial-period square-wave surface-relief grating in amorphous $\mathrm{SiO}_{2}$ showing that the grating has induced an oriented crystal growth where $\langle 100>$ directions are parallel to the grating grooves. The grooves are $25 \mathrm{~nm}$ deep and have the lighter shading. The electron microscopy produces some decomposition of the KCl crystallites during viewing leading to the serrated edges of some of the crystallites. 


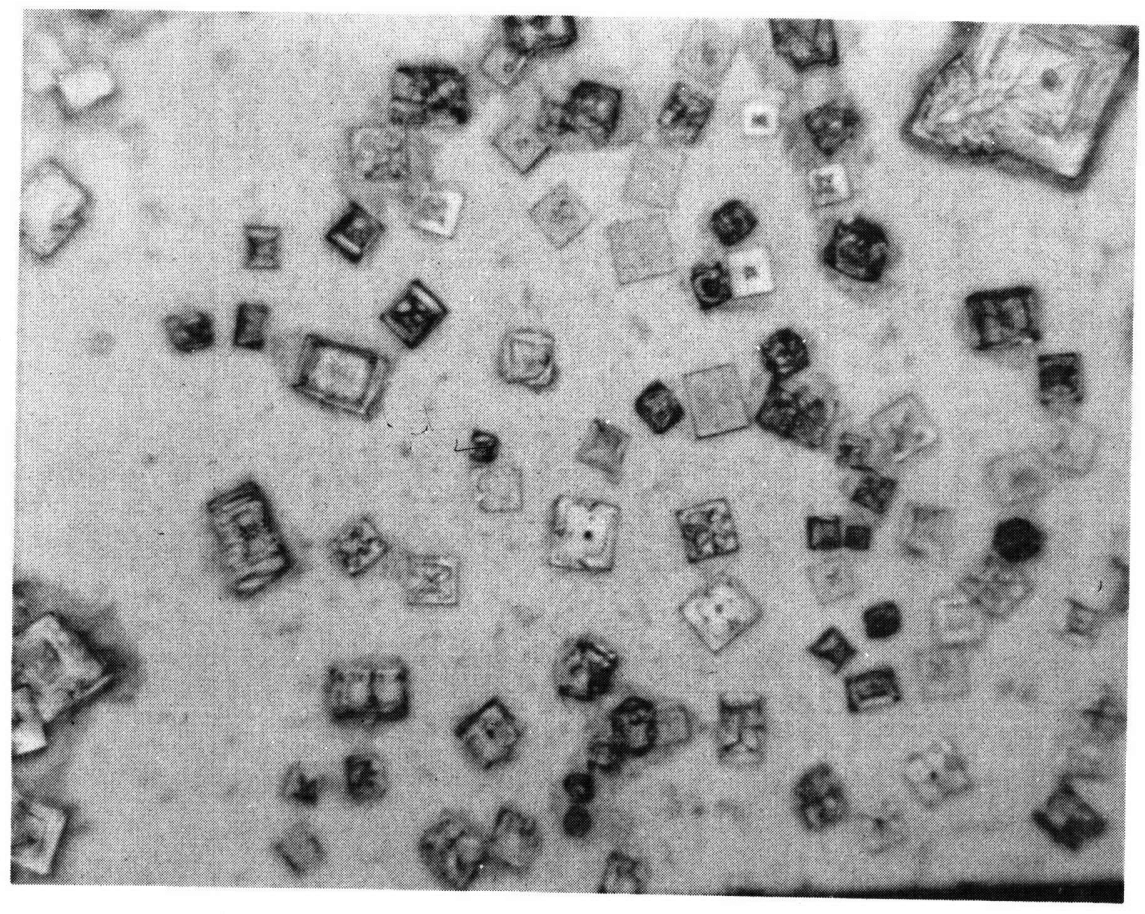
Fig. 5-24. An optical micrograph of $\mathrm{KCl}$ crystallites grown on a smooth $\mathrm{SiO}_{2}$
surface. The magnification is $500 \mathrm{X}$. 


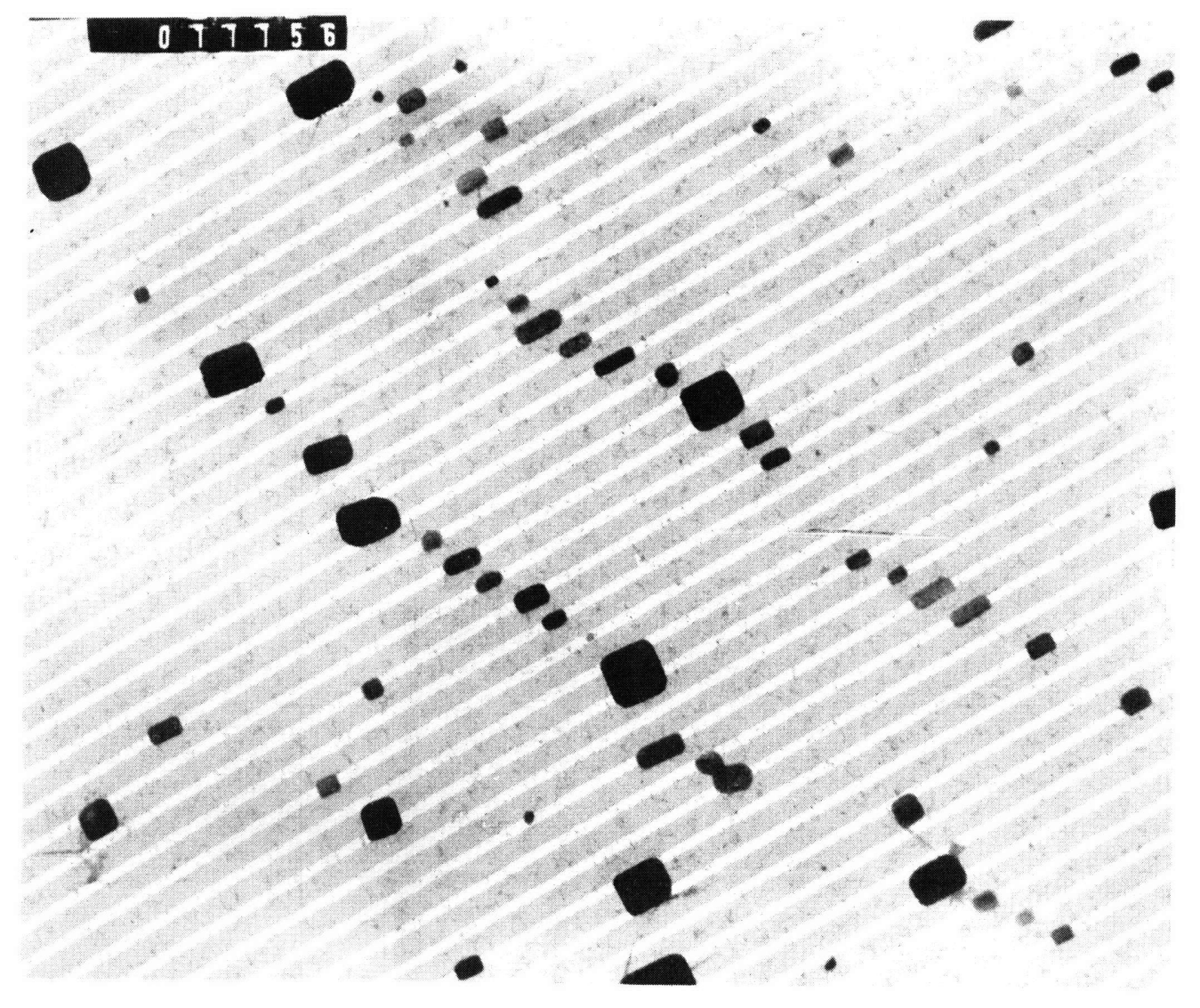

Fig. 5-25. Three transmission electron micrographs of different areas on the same substrate are shown of $\mathrm{KCl}$ crystallites grown on a $320 \mathrm{~nm}$ spatial-period square-wave surface-relief grating in amorphous $\mathrm{SiO}_{2}$. The grooves are $25 \mathrm{~nm}$ deep and have the lighter shading. a.) The first area viewed. 


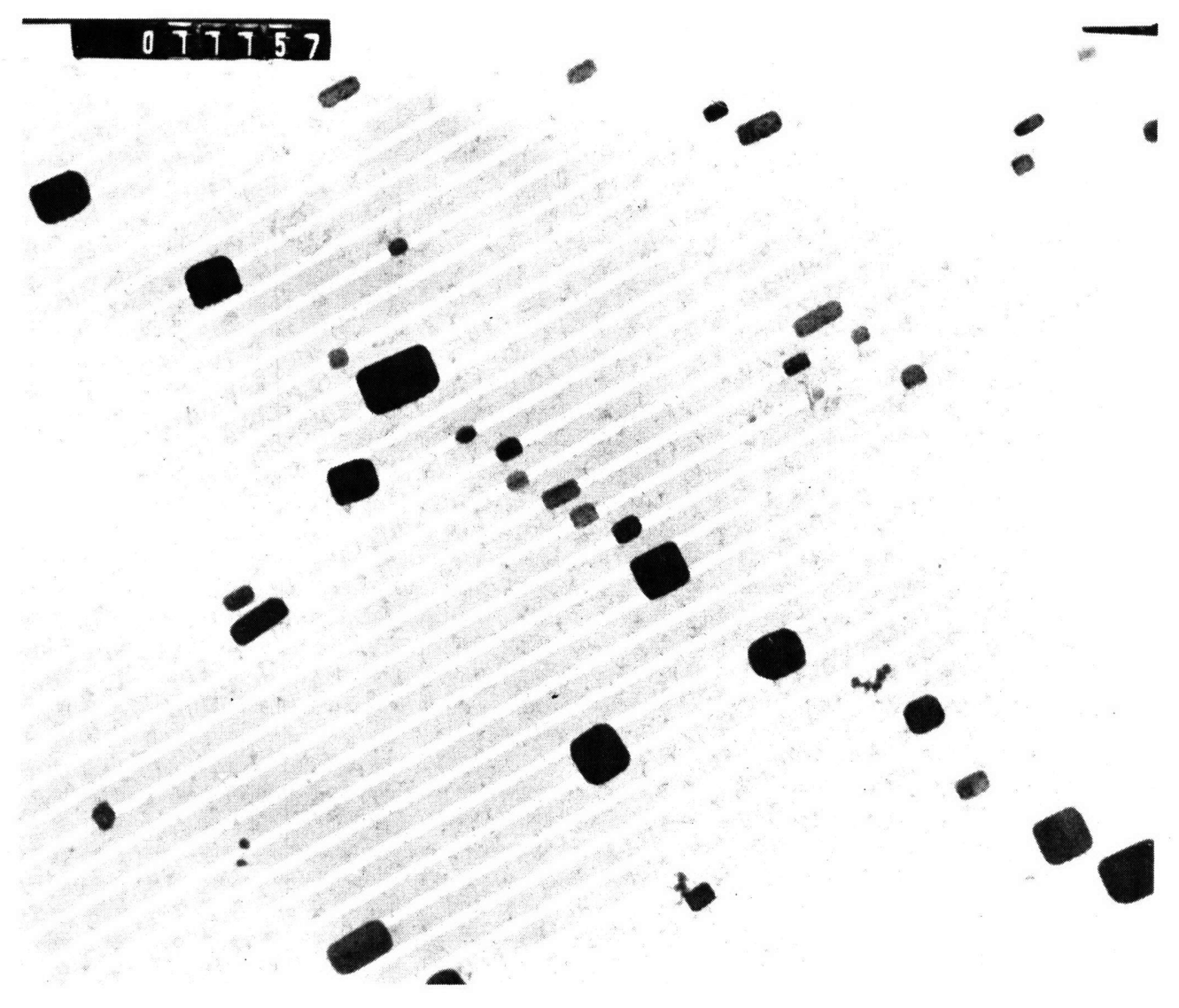

Fig. 5-25. Three transmission electron micrographs of different areas on the same substrate are shown of $\mathrm{KCl}$ crystallites grown on a $320 \mathrm{~nm}$ spatial-period square-wave surface-relief grating in amorphous $\mathrm{SiO}_{2}$. The grooves are $25 \mathrm{~nm}$ deep and have the lighter shading. b.) The second area viewed. 


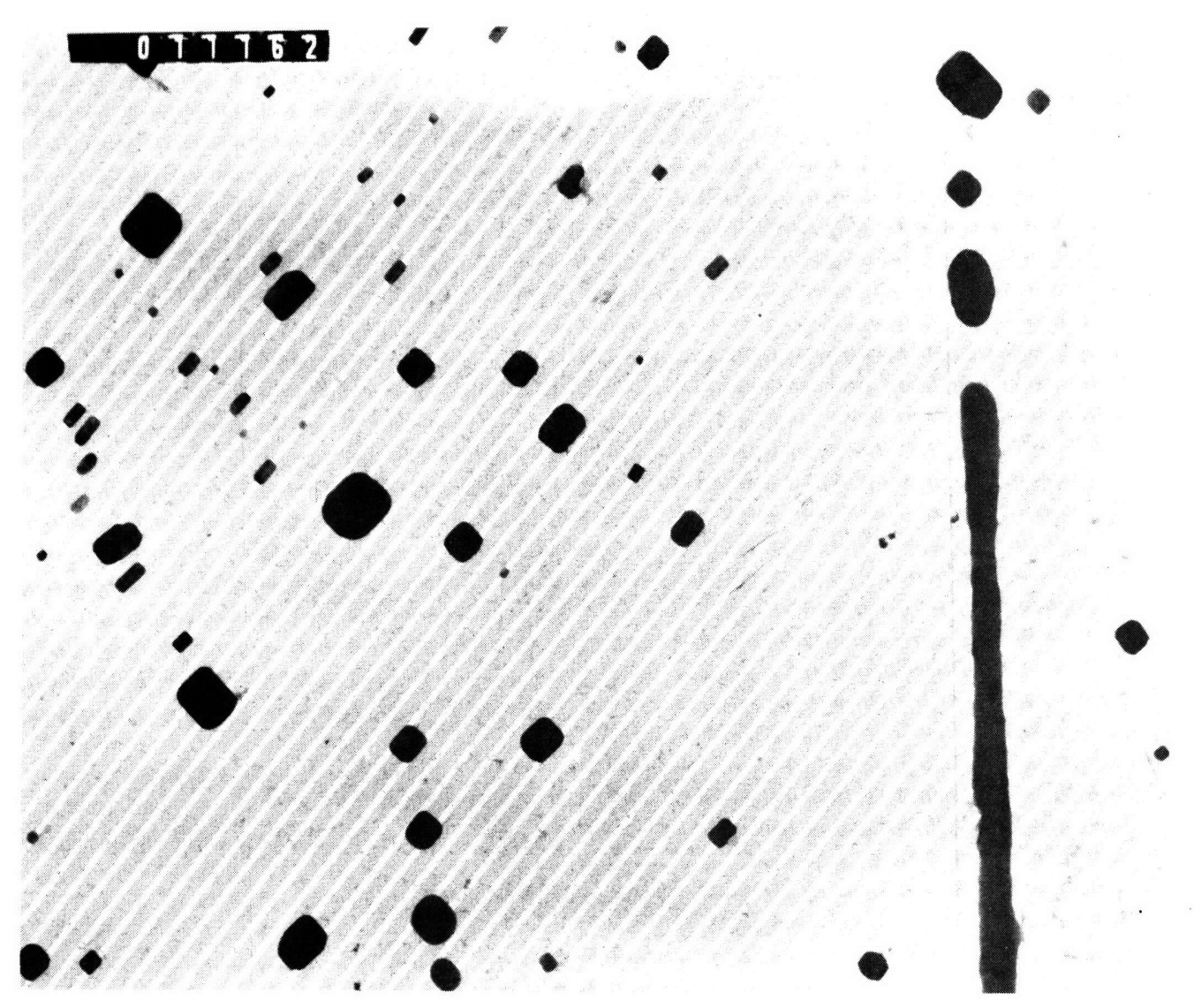

Fig. 5-25. Three transmission electron micrographs of different areas on the same substrate are shown of $\mathrm{KCl}$ crystallites grown on a $320 \mathrm{~nm}$ spatial-period square-wave surface-relief grating in amorphous $\mathrm{SiO}_{2}$. The grooves are $25 \mathrm{~nm}$ deep and have the lighter shading.

c.) The third area viewed. Very long "dendritic" crystallites such as seen here in (c.) tend to break up into small crystallites during annealing. 


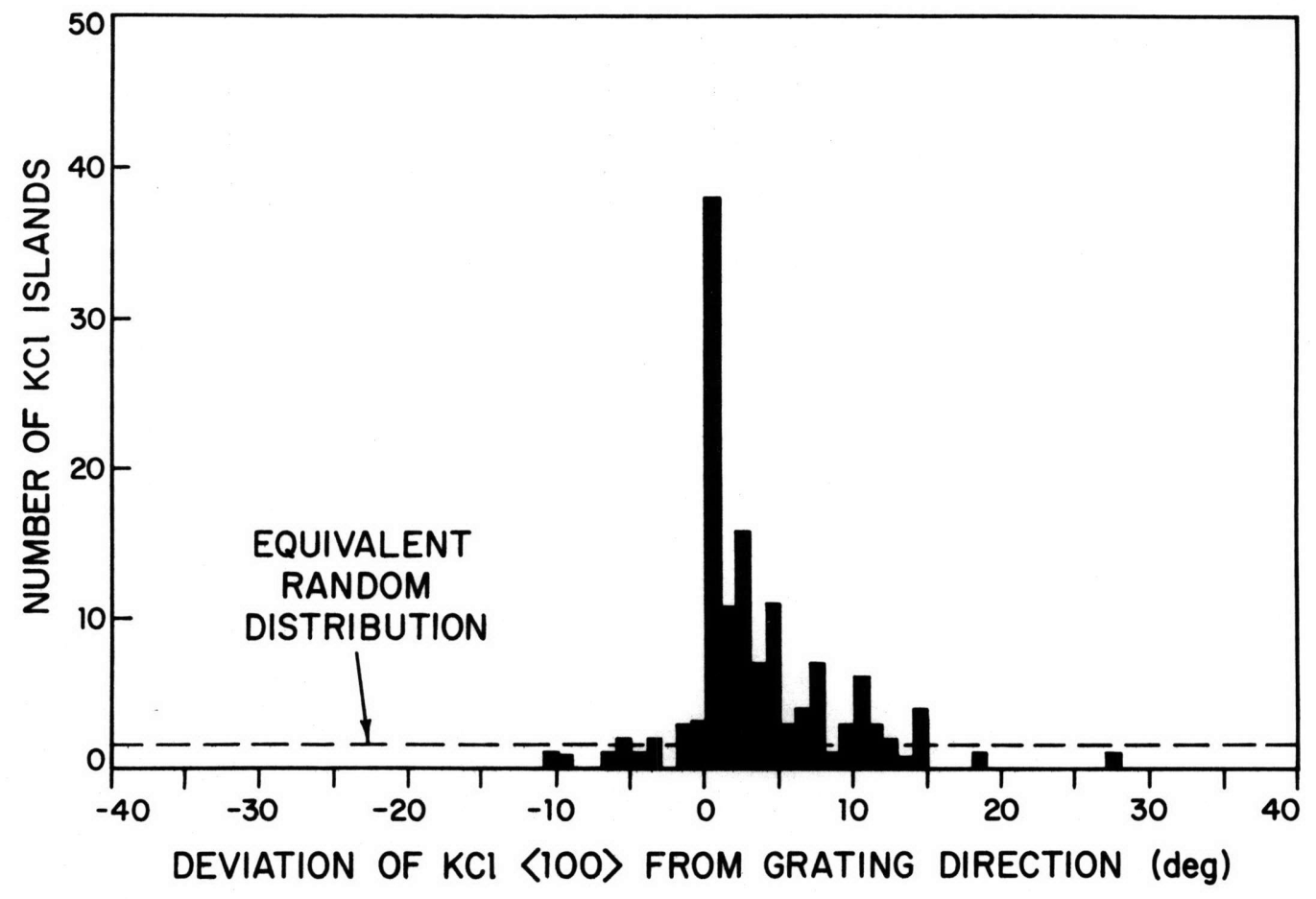

Fig. 5-26. A plot is shown of the measured deviation of the $\langle 100\rangle$ direction of the $\mathrm{KCl}$ crystallites from the grating groove direction for the microaraphs shown in Fig. 5-25. 
groove corners. (The smallest $\mathrm{KCl}$ islands seen in micrographs of depositions have been $\approx 200 \AA$ in length.) Depositions performed on surface-relief structures where $25 \mathrm{~nm}$ high steps were spaced 1-3 $\mu \mathrm{m}$ apart versus the $0.16 \mu \mathrm{m}$ spacing of the structure in Fig. 5-23 exhibited "decoration" of the steps where essentially all of the KCl crystallites were located at the steps. An optical micrograph of such an experiment is shown in Fig. 5-27.

An attempt was made to determine if the observed "decoration" effect where virtually all of the KCl islands occur at steps could be destroyed by increasing the radius of the groove corners of the gratings structure. The original square profile of the substrate used for the deposition shown in Fig. 5-23 was degraded by removing $15 \mathrm{~nm}$ of $\mathrm{SiO}_{2}$ with an isotropic aqueous chemical etch. ("P-etch" $15 \mathrm{ml} \mathrm{HF}, 10 \mathrm{ml} \mathrm{\textrm {HNO } _ { 3 }}$ and $300 \mathrm{ml} \mathrm{H}_{2} \mathrm{O}$ for $60 \mathrm{sec}$. was used) ${ }^{1}$. The new radius $\mathrm{Rg}$ could not be measured directly but is expected to be $>15 \mathrm{~nm}$. A KCl deposition was performed on the degraded structure. Micrographs of the result are shown in Fig. 5-28. A strong "decoration" effect is still present and nearly all of the $\mathrm{KCl}$ islands occur at the steps. However, there was a major change in morphology relative to Fig. $5-23$, the orientation effect appears to have been seriously degraded. Few of the $\mathrm{KCl}$ crystallites are oriented with the grating groove direction. This lack of strong orientation was observed over the entire sample. The sample was "annealed" by placing it in a humid atmosphere for several hours. The sample was then reexamined in the STEM. A micrograph of the annealed sample is shown in Fig. 5-28(b). During the annealing the crystallographic shape of the $\mathrm{KCl}$ islands became better defined but 


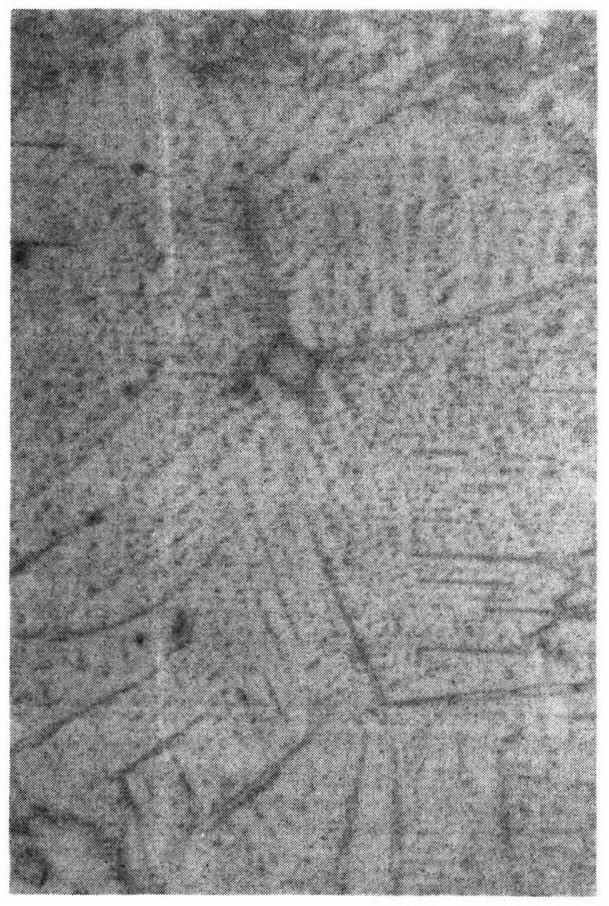

Fig. 5-27. Optical micrograph at 1000X of a $\mathrm{KCl}$ deposition on a squarewave surface-relief structure in $\mathrm{SiO}_{2}$ with an irregular step spacing which in some areas is as large as $3 \mu \mathrm{m}$ Close examination reveals that nearly all of the small nuclei are confined to the steps. 


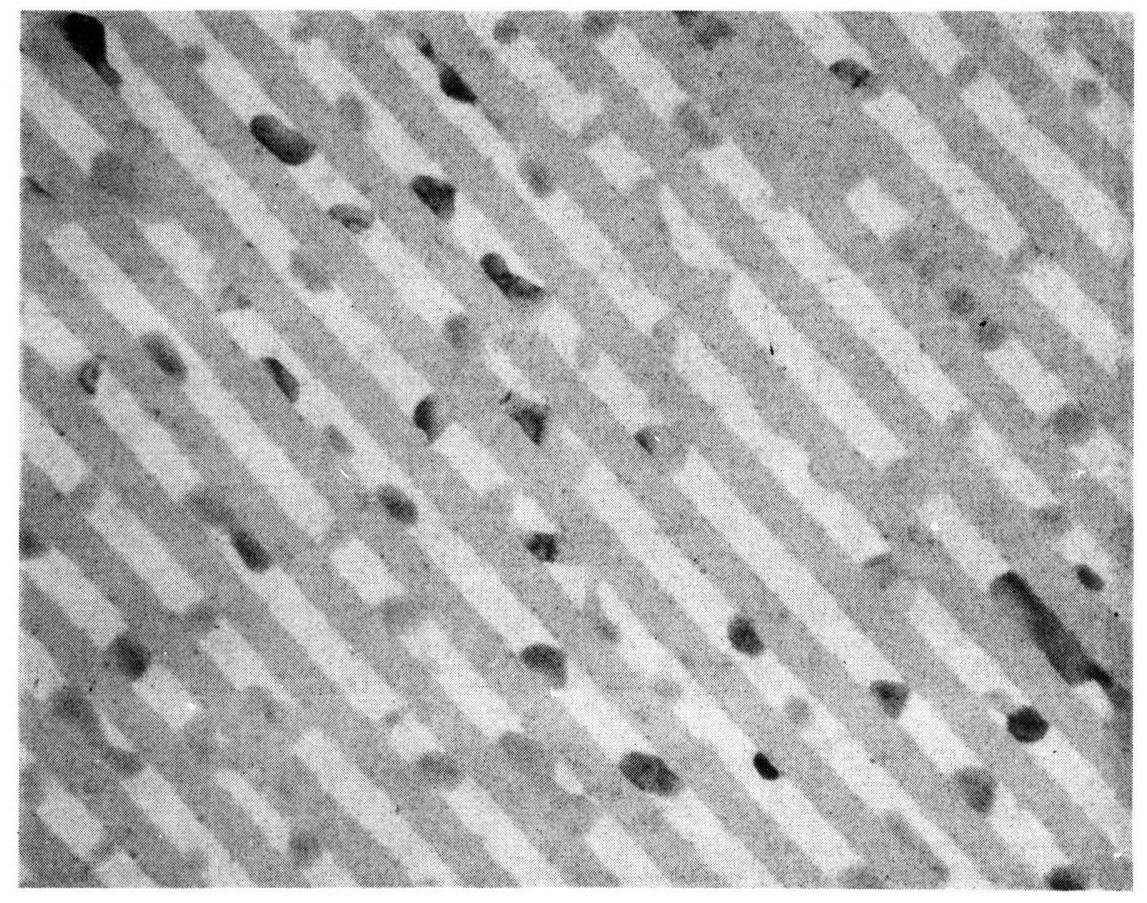

Fig. 5-28. Scanningatransmission-electron micrographs of a $\mathrm{KCl}$ deposition on a square-wave structure which has been degraded by isotropically etching $150 \AA$ of $\mathrm{SiO}_{2}$ from the structure shown in Fig.5-23.

a.) Immediately after deposition. 


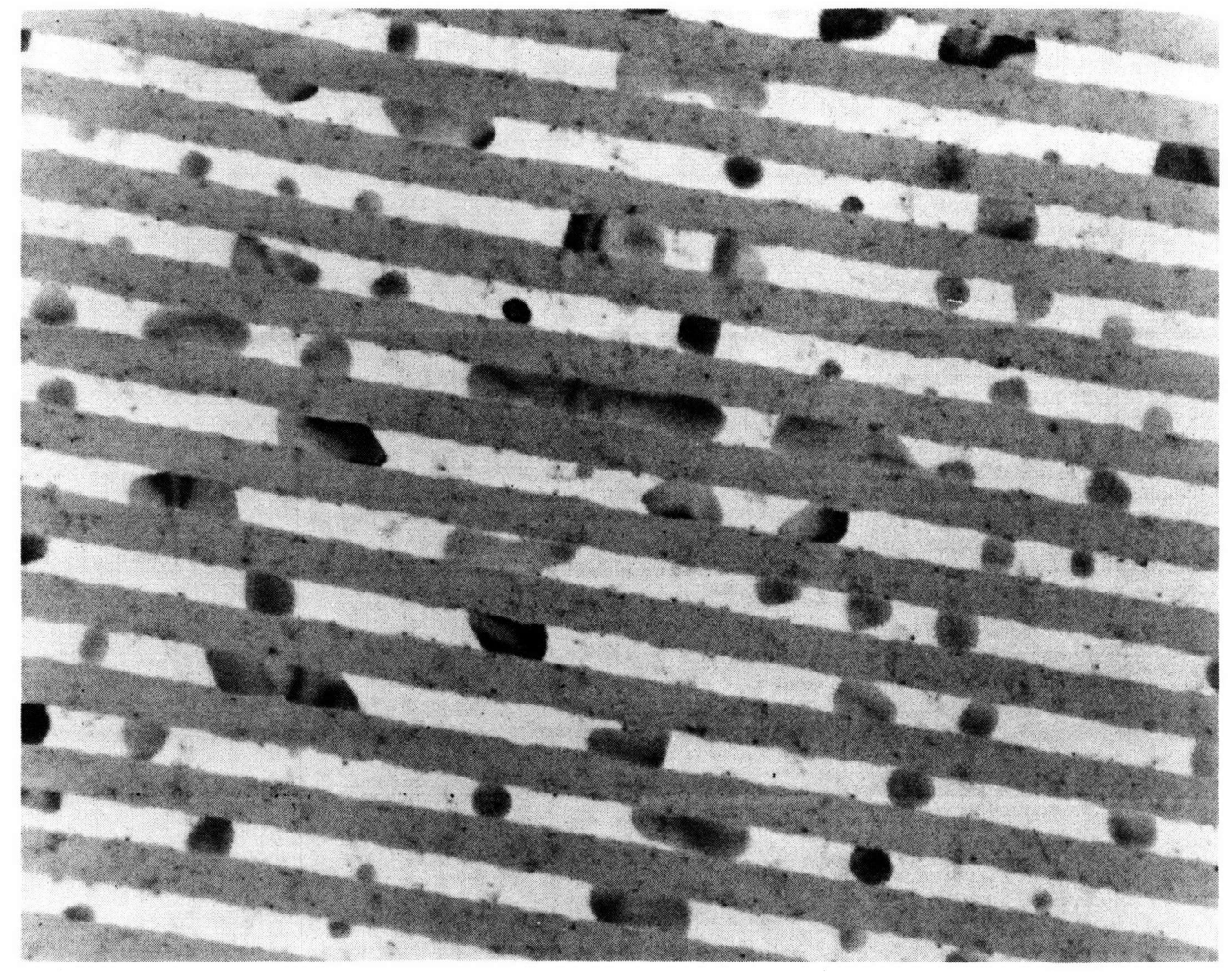

Fig. 5-28. Scanning-transmission-electron micrographs of a $\mathrm{KCl}$ deposition on a square-wave structure which has been degraded by isotropically etching $150 \AA$ of $\mathrm{SiO}_{2}$ from the structure shown in Fig. 5-23.

b.) After a 2 hour "anneal" in a humid atmosphere. 
it appears that the orientation did not significantly improve. Attempts to further degrade the square profiles by removing more $\mathrm{SiO}_{2}$ by isotropic chemical etching failed because further reduction in the thickness of the $\mathrm{SiO}_{2}$ layer weakened the thin samples to the point where they broke.

It appears that the degraded $\mathrm{SiO}_{2}$ structure induces some sort of "decoration" effect but does not induce oriented growth. This would be expected if the curvature of the grooves was still small enough to induce decoration but the structure did not provide sufficiently well defined facets such that a strong minimum in the interfacial tension was obtained at the $<100>$ orientation.

It was observed that large crystallites which are sometimes formed during $\mathrm{KCl}$ deposition tend not to be oriented with respect to the grating structure. This phenomenon can be seen in Fig. 5-27 where the large $(\approx 10 \mu \mathrm{m})$ crystallite in the center of the micrograph is not oriented. The large crystallites are most likely the first ones which nucleate. It is possible that these larger islands are not oriented because they nucleate on dirt particles in the solution or on the substrate at relatively low supersaturations early in the deposition. Another possibility is that all islands large and small nucleate randomly and acquire their orientation at a later stage. The large crystallites would be less likely to reorient than the small crystallites. Significant improvement of the orientation of $\mathrm{KCl}$ deposits by annealing has not been observed. However, there is an unavoidable delay between the time of deposition and the time of observation and annealing may occur spontaneously before the deposit is observed. 
In conclusion the available data strongly indicates that oriented nucleation and growth of $\mathrm{KCl}$ deposited from aqueous solution occurs on square-wave grating surface relief structures on amorphous $\mathrm{SiO}_{2}$ substrates. Preferential nucleation appears to occur on degraded square wave structures but oriented nucleation and growth does not appear to occur. Some questions remain about the role of unavoidable annealing of the deposit. Irrespective of the exact details of the mechanisms which lead to orientation the fact remains that a $\mathrm{KCl}$ deposit has been oriented by a square-wave grating on an amorphous substrate with the minimum free energy orientation predicted by the simple models of Chapter II and section 5.3.2. In effect, heteroepitaxy has been induced on an amorphous substrate by the introduction of an "anisotropic" surface structure. 


\subsubsection{TIN DEPOSITIONS}

The alkali halides can be deposited by thermal evaporation in a high vacuum. Because of the success which was achieved with $\mathrm{KCl}$ deposited from solution it would be a natural choice for thermal evaporation deposition experiments. Unfortunately this could not be done because it was believed that the presence of $\mathrm{KCl}$, which is hydroscopic, in a vacuum system would lead to its deterioration. For this reason thermal evaporation deposition experiments were done with tin which is more compatible with good vacuum practice.

Tin has a tetragonal structure (at room temperature) which exhibits a very strong fiber texture when deposited on smooth $\mathrm{SiO}_{2}$ substrates where $<100>$ is normal to the substrate surface. ${ }^{78}$ (This orientation represents the most densely packed plane parallel to the substrate). A tetragonal material which exhibits such a texture is ideally suited for orientation by a square-wave grating because the equilibrium orientation is unique and should occur for the $<001>$ direction parallel to the groove direction.

To study the growth of tin a series of depositions by electron beam evaporation in high vacuum onto square-wave gratings in $\mathrm{SiO}_{2}$ were performed. The structures were fabricated in precisely the same way as those used for the $\mathrm{KCl}$ deposition experiments discussed in section 5.4.3 Depositions were done in which the average tin thickness was $0.8 \mathrm{~nm}, 4 \mathrm{~nm}, 40 \mathrm{~nm}$ and $80 \mathrm{~nm}$. Micrographs of the results are shown in Figures 5-29,5-30,5-31 and 5-32 respectively. The deposition rate was $\approx 1 \AA$ $/ \mathrm{sec}$. for the $0.8 \mathrm{~nm}$ and $4 \mathrm{~nm}$ 


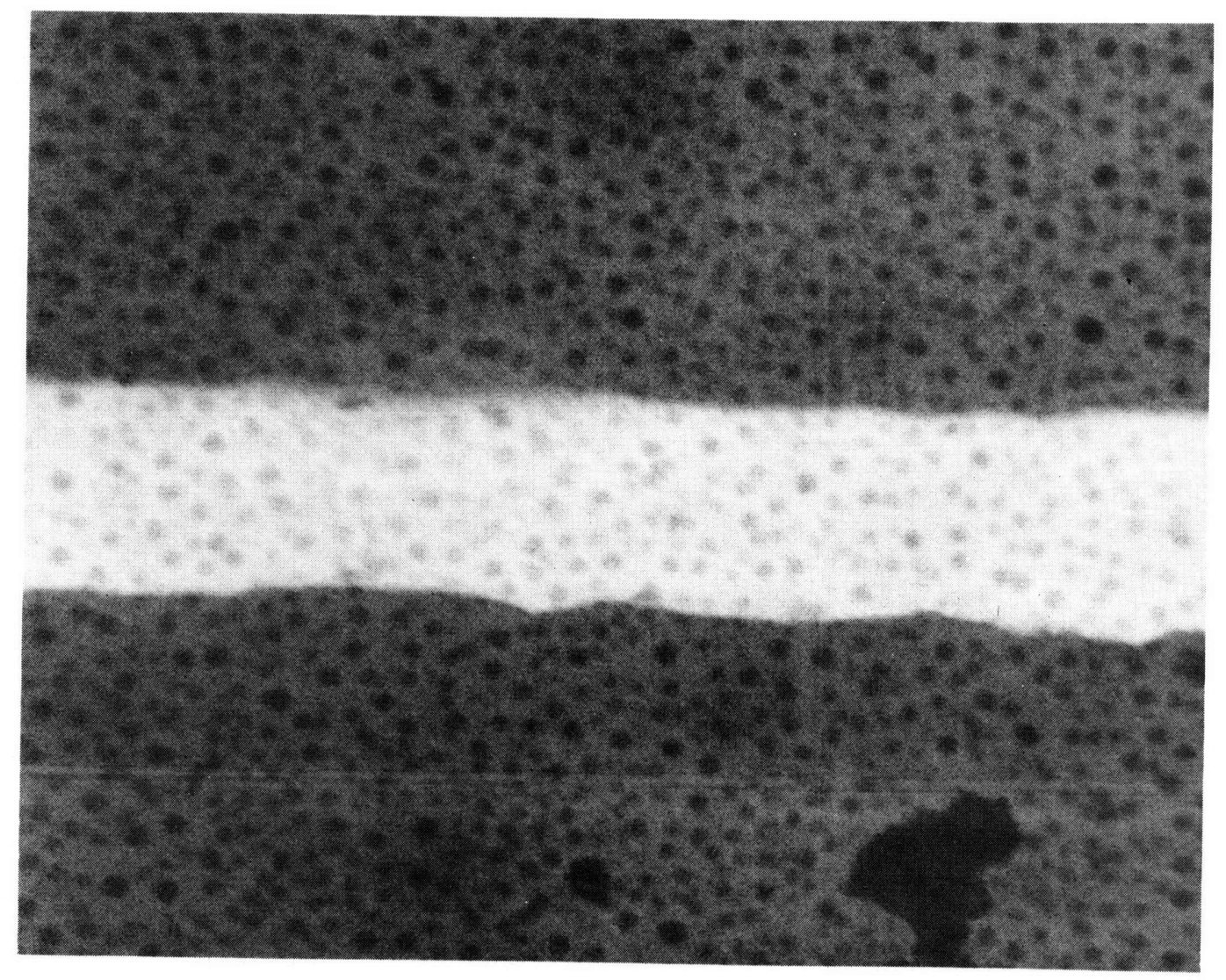

Fig. 5-29. A scanning-transmission-electron micrograph of an $0.8 \mathrm{~nm}$ average thickness tin deposit on a $50 \mathrm{~nm}$ deep $320 \mathrm{~nm}$ period square-wave grating in amorphous $\mathrm{SiO}_{2}$. The lighter area is a grating groove. The
magnification is $\approx 215 \mathrm{KX}^{2}$. 

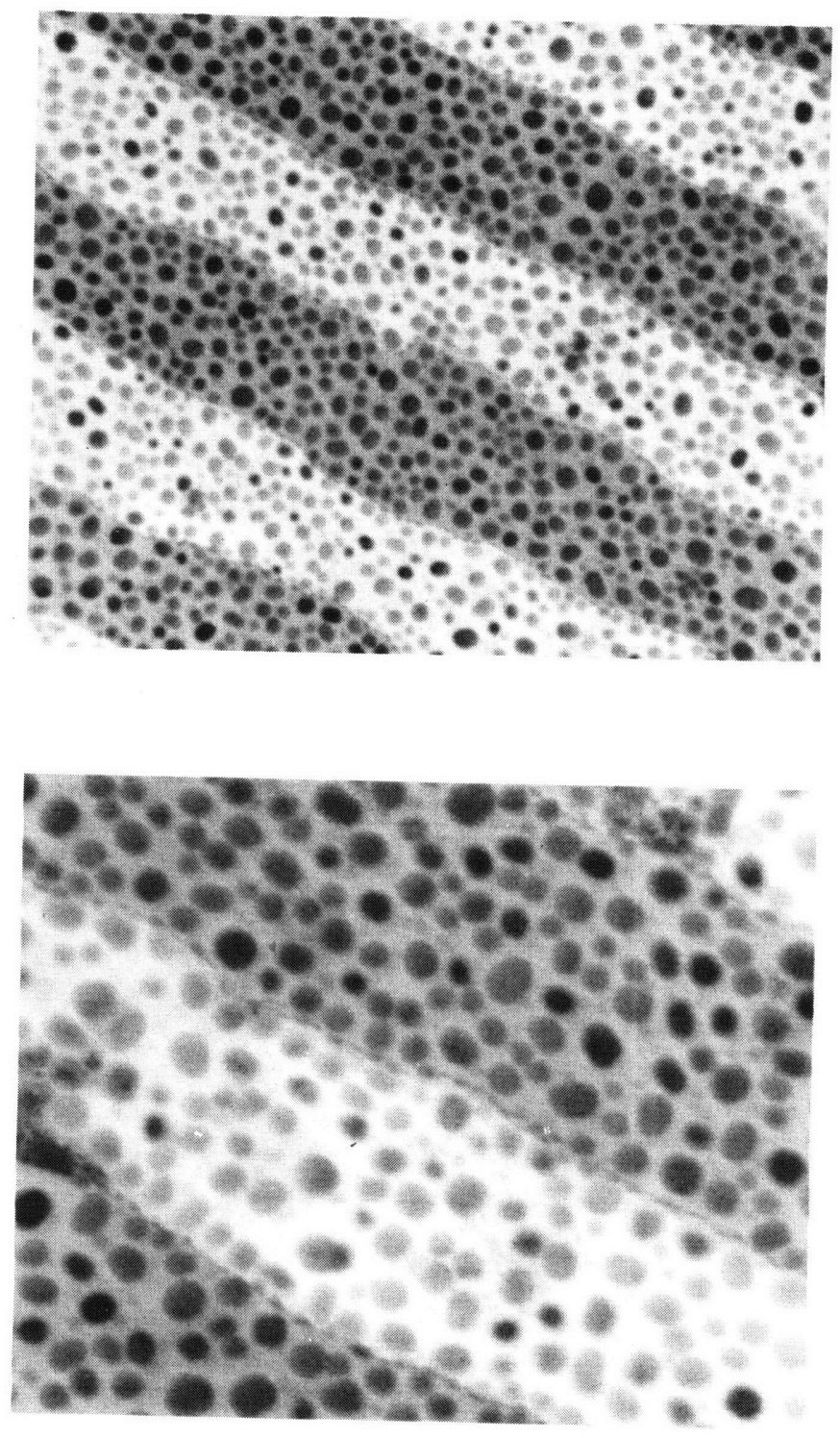

Fig. 5-30. Scanning transmission electron micrographs of a $4 \mathrm{~nm}$ average thickness tin deposit on a $50 \mathrm{~nm}$ deep, $320 \mathrm{~nm}$ period square-wave grating in amorphous $\mathrm{SiO}_{2}$. Some perferential nucleation appears to have occured at the steps. The magnification is $\approx 150 \mathrm{KX}$ for the upper micrograph and $\approx 300 \mathrm{KX}$ for the lower micrograph. 


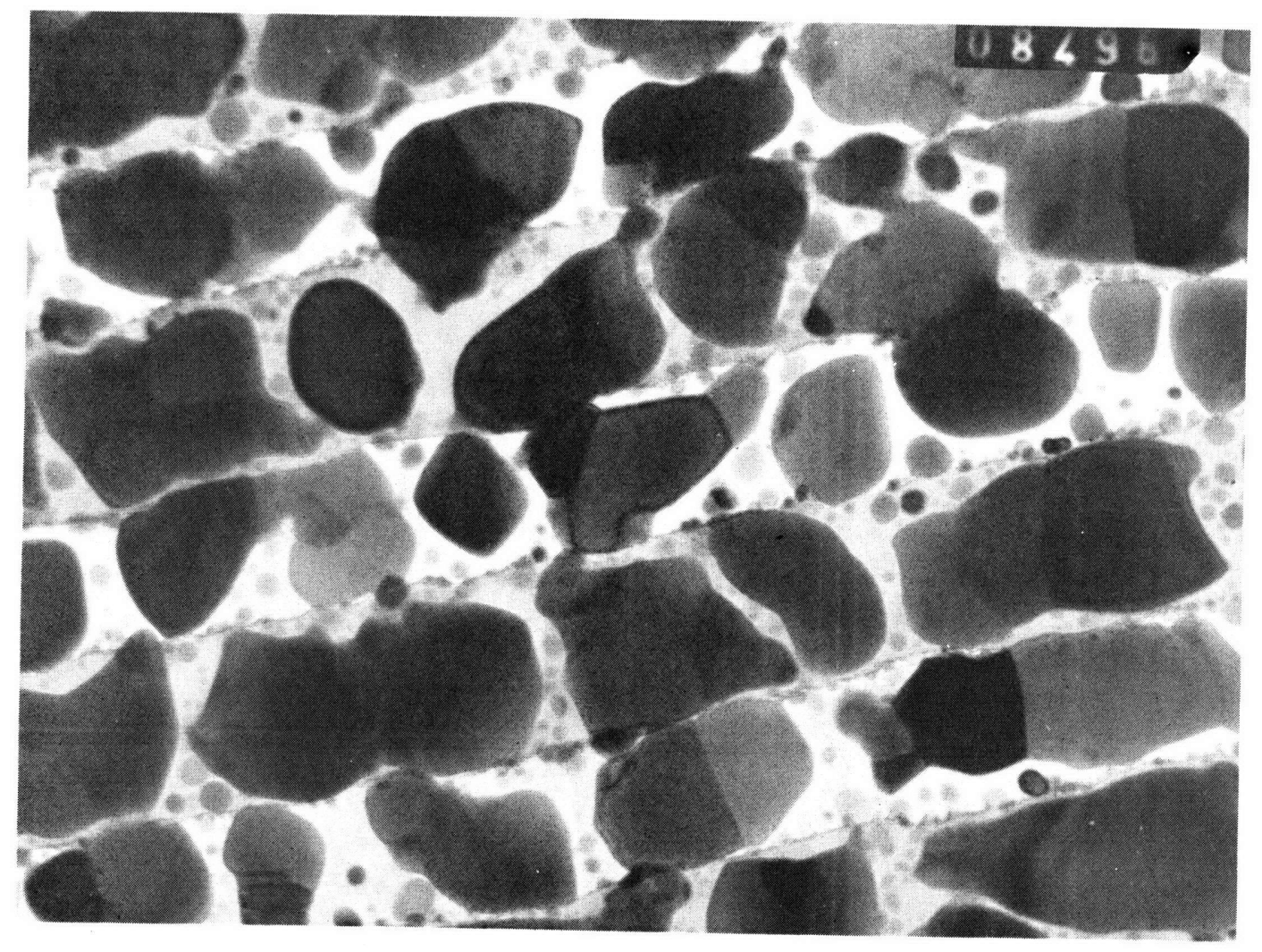

Fig. 5-31. A transmission electron micrograph of a $40 \mathrm{~nm}$ average thickness tin deposit on a $50 \mathrm{~nm}$ deep $320 \mathrm{~nm}$ period square-wave grating in amorphous $\mathrm{SiO}_{2}$. The magnification is $\approx 120 \mathrm{KX}$. 


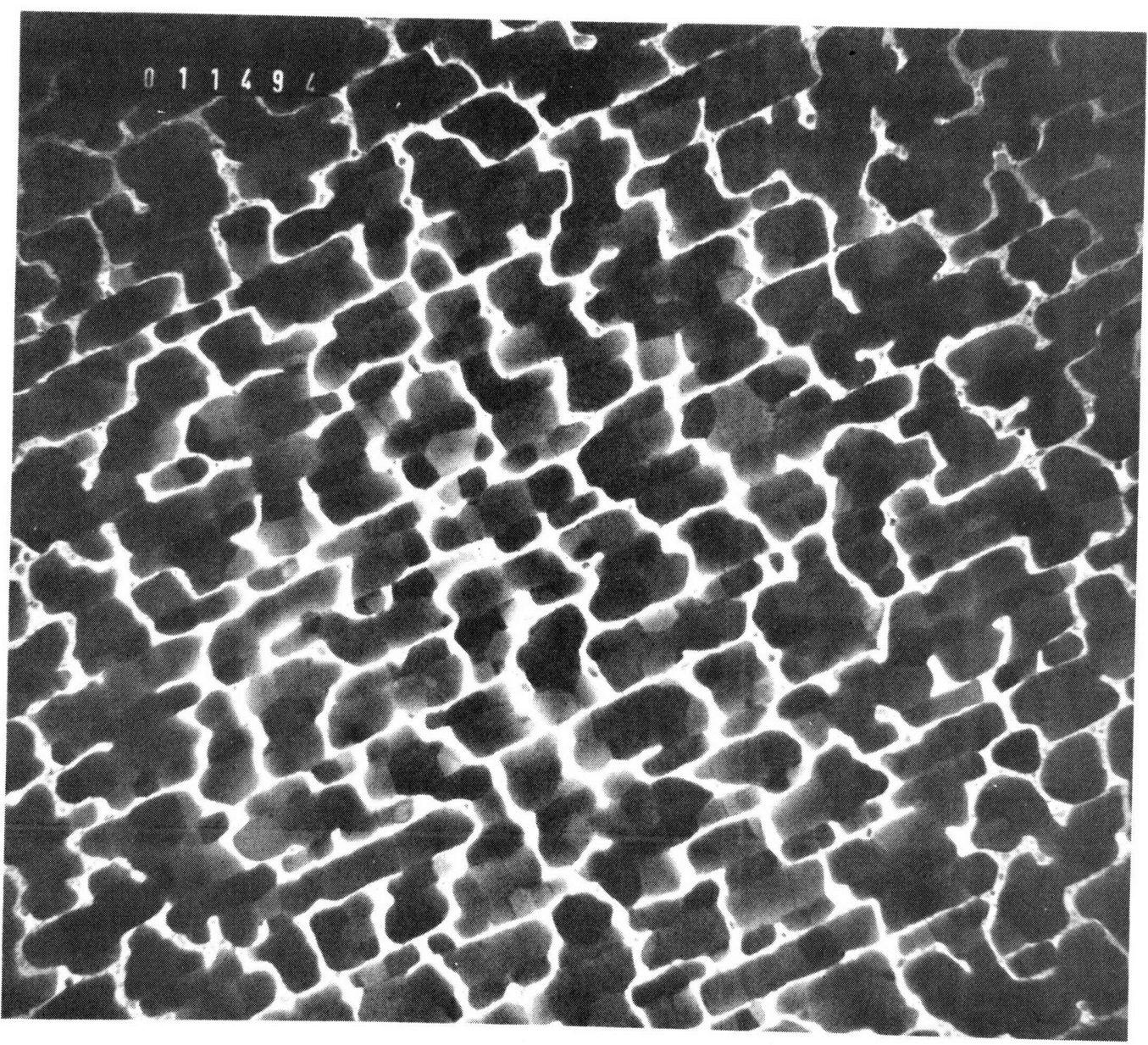

Fig. 5-32, A transmission electron micrograph of an $80 \mathrm{~nm}$ average thickness tin deposit on a $50 \mathrm{~nm}$ deep, $320 \mathrm{~nm}$ period square-wave grating in amorphous $\mathrm{SiO}_{2}$. The film has entered the early network stage of film growth and recrystallization appears to be slow. 
thick depositions and (1-5) $\AA$ /sec for the $40 \mathrm{~nm}$ and $80 \mathrm{~nm}$ thick depositions. The depositions were performed with the substrate at room temperature.

Growth appears to proceed by the sequence described in section 5.3.2 and depicted in Fig. 5-15 or Fig. 5-16. As seen in Figures 5-29 and 5-30 significant decoration of the steps does not occur at the early stages of growth. (A weak decoration effect can be detected in Fig. 5-30.) This is consistent with the results obtained with gold since the size of the critical nuclei should be comparable for gold and tin deposited in vacuum by thermal evaporation. As growth proceeds the islands tend to recede from the steps by the coalescence phenomena discussed in section 5.3 .2 and illustrated in Fig. 5-16. For this reason the islands do not experience the orienting influence of the steps until they have grown large enough to touch the step walls. Examination of the micrographs shown in Figures 5-31 and 5-32 indicates that the network stage of film growth occurs before the islands grow to touch the walls and the orientation of the islands has been strongly affected. This situation, depicted in Fig. 5-16(e), makes the appearance of a single crystal film unlikely because reorientation does not appear to occur for the large islands resulting from the network formation. However, the morphology of the islands which do touch the walls in the $80 \mathrm{~nm}$ thick film shown in Fig. 5-32 indicates that the tin film may have asquired some preferred orientation. Reflection electron diffraction (RHEED) of the deposit revealed that the $\{100\}$ planes of the crystallites are parallel to the substrate (as expected) but no in plane orientation was found. Because of the grazing incidence of the electron beam used, 
RHEED only samples the uppermost layers of the deposit. Oriented islands between the grooves might not be detected. Selected area electron diffraction of the $40 \mathrm{~nm}$ thick deposition where few islands touch the walls showed no preferred in plane orientation. Selected area diffraction of the $80 \mathrm{~nm}$ thick deposit where most of the islands do touch the step walls was difficult because of its high electron attenuation. Some of the diffraction patterns which were obtained indicated that a major crystallographic direction of some of the islands of the deposit was aligned with the grating direction. An example of a selected area and the corresponding diffraction pattern are shown in Fig. 5-33. Because only a partial spot pattern was obtained and other spots are present unambiguous determination of the deposit orientation from the diffration pattern is difficult. The spots in Fig 5-33(b) which are indicated correspond to $\{100\}$ parallel to the substrate and <001> parallel to the grating direction. This incomplete spot pattern can be compared with the complete pattern shown in Fig. 5-9 for the same orientation of tin. The diffration patterns obtained from the $80 \mathrm{~nm}$ thick tin deposition sugqest that some weak preferred orientation has been induced by the square wave structure.

If the square-wave grating structure is to exert a stronger orienting influence, the grating must orient the tin islands before the network stage is reached. Thus, the grating period should be smaller and/or the corners of the structure should be sharper so as to induce strong decoration effects. It is also possible that careful control of deposition parameters and/or annealing could lead to orientation using the 

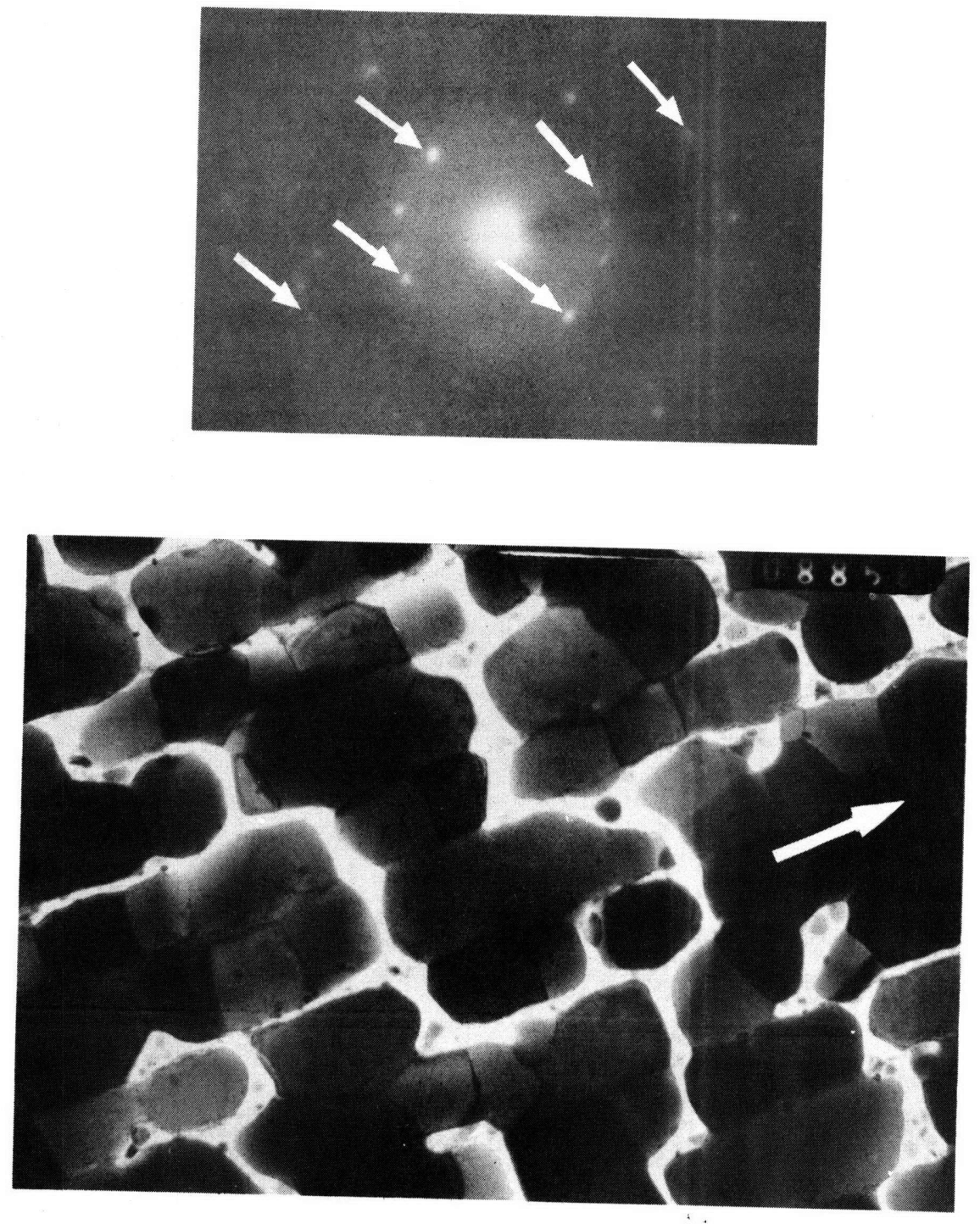

Fig. 5-33. A TEM micrograph of the $80 \mathrm{~nm}$ average thickness tin deposition and the corresponding selected area electron diffraction pattern are shown. The arrows on the diffraction pattern indicate spots which could originate from crystallites with $\{100\}$ parallel to the substrate and $<001>$ parallel to the grating groove direction. The arrow on the image indicates the grating groove direction. 
existing structures or that grid structure such as the one depicted in

Fig. 5-13 could be used to avoid onset of the network stage before the steps induced reorientation of the growing islands. 
VI. CONCLUSION

\subsection{SUMMARY OF WORK}

A new method of orienting crystalline overlayers on amorphous substrates by artificially-produced surface-relief structures was investigated. A simple model indicated that demonstration of the predicted orientation effects would require submicrometer-dimension surface-relief structures with well controlled profiles. Existing fabrication technology was found to be inadequate to define such structures. To solve this problem new fabrication techniques were developed inclüding; very soft $X$-ray 1 ithographic techniques and reactive-ion-etching. With these new techniques $320 \mathrm{~nm}$ period square-wave gratings in $\mathrm{SiO}_{2}$ were fabricated. These simple structures were used to investigate orientation effects in several overlayer systems. Uniform single crystal orientation was induced in nematic and smectic A liquid crystals. The observed orientation of the liquid crystals agreed with the model presented here, and with an earlier model of Berreman's. Potassium chloride (KCI) crystallites deposited from aqueous solution on square-wave gratings were aligned with $\langle 100\rangle$ directions parallel to the groove directions and perpendicular to the substrate as predicted by a detailed model of the thin film growth process. Potassium chloride (KCl) crystallites appear to have preferentially nucleated at vertical steps in the grating. Square-wave grating structures which had been degraded by isotropic etching still induced "decoration" effects but failed to induce orientation of $\mathrm{KCl}$ crystallites. Depositions of gold and tin by vacuum evaporation onto the square-wave grating structures exhibited only 
very weak decoration effects. This could be explained by the fact that the radii of the corners of the square-wave structures are larger than the radii of the critical nuclei of gold and tin. A series of tin depositions of increasing thickness were done on a square-wave structure. The growth proceeds by random nucleation followed by a coalescence stage where the tin islands recede from the steps. Eventually, the islands grow to the point where they contact the steps, at this stage some weak orientation appears to have been induced. Before large scale orientation of the tin is achieved, a network of islands is formed and reorientation is inhibited. This result is explained by a detailed model of thin film growth on square-wave structures. It is proposed that improved surface relief structures are needed to achieve strong orientation effects for vacuum evaporated films.

These experiments show that the concept of orienting crystalline overlayers on amorphous substrates by surface relief structures has been clearly demonstrated in the cases of the liquid crystals MBBA and M-24, and for potassium chloride deposited from aqueous solutions. A series of experiments with gold and tin have yielded very useful information about the sequence of nucleation, growth, and coalescence on surface relief structures. The results with tin indicate that the orientation of tin islands has been weakly affected and that by reducing the period of the surface relief structure single crystal orientation should be achieved. 


\subsection{PROPOSALS FOR FUTURE WORK}

It is believed that this new approach of manipulating overlayers with an artificially produced substrate surface-relief structure should open a new field of investigation. A few new experiments which are suggested by the results obtained in this thesis research are presented here.

Many more materials and deposition techniques could be employed in further orientation experiments on the simple square-wave gratings in $\mathrm{SiO}_{2}$ used for the orientation effects reported here. A great many cubic and tetragonal crystalline materials are known to exhibit $\{100\}$ and $\{110\}$ $1,2,3$

nucleation deposition and annealing fiber textures. These are obvious candidates for further experiments with square-wave gratings. Of particular interest may be the study of annealing of overlayers of these materials deposited under conditions such that the overlayer is initially amorphous or very small grained polycrystalline.

The fabrication techniques already developed are very versatile and immediately have a wide area of possible applications including integrated optics, integrated circuits, and a host of planar devices requiring submicrometer structures. Crystalline overlayer orientation experiments provide an excellent framework for developing and improving fabrication techniques because the structures required for orienting crystalline overlayers provide very stringent and well defined goals for technology improvement.

If smaller structures are required to orient vapor deposited overlayers there appears to be no fundamental problem. $83.5 \mathrm{~nm}$ period gratings in PMMA have already been defined by Bjorklund using holographic lithography. 
Extensions of the holographic lithography mask pattern generation technique or recently developed contamination electron beam writing should yield grating type mask patterns with linewidths of less than $10 \mathrm{~nm}$. As discussed in Chapter III, $C_{k} 4.48 \mathrm{~nm} X$-ray $1 \mathrm{ithography}$ using PMMA should have the resolution required to replicate such patterns. Before such linewidths are used a great deal can be done to improve the quality of $160 \mathrm{~nm}$ linewidth square wave gratings in amorphous $\mathrm{SiO}_{2}$. Grating sidewall smoothness and groove straightness could be improved by improvements in X-ray mask quality, improvements in the liftoff process, and a better understanding of the reactive-ionetching process.

Obviously, the ability to fabricate structures other than square wave gratings is desirable. The extension of the techniques presented here to the fabrication of grids and other patterns which are superpositions of gratings is straightforward. Techniques for fabricating sawtooth structures with smooth facets at specified angles are less obvious. At the present there is a great deal of interest in the development of fabrication technology for small structures. This new application should provide an important testing ground.

A goal of this work has been to produce large area single crystal films, for some applications, notably integrated circuits, a large area single crystal film is not required. The individual components of an integrated circuit actually occupy very small volumes of semiconductor and must be electrically isolated from one another. If isolated single crystal islands several square micrometers in area could be deposited on an insulating 
substrate, this should be sufficient to fabricate an integrated circuit. A possible way of achieving this is illustrated in Fig. 6-1. Square pits could be etched into an amorphous substrate using a chrome etch mask which has been defined using X-ray lithography and liftoff. Silicon could then be evaporated or sputtered over the entire substrate. The silicon in the pits should assume a single crystal orientation within each pit under the proper deposition conditions as discussed in Chapter $V$. Subsequently the superfluous silicon could be 1 ifted off by chemically removing the chrome.

The remarkable success achieved in orienting liquid crystals makes possible a wide variety of experiments. It has been estimated that approximately $0.5 \%$ of the organic molecules exhibit liquid crystal mesophases. A tremendous number of materials are thus available for experimentation. Since the geometry of the structures can be directly controlled and the orientation mechanisms are understood, one could possibly use surface relief structures to organize liquid crystal molecules for some purpose. As an example, one might want to study a uniform array of disclinations in a nematic liquid crystal or an array of "myelinic" textures in a material exhibiting the smectic A phase. Remembering that bend and twist are forbidden in a smectic $A$ and that distortions must be pure splay, one sees that a sandwich of substrates with an array of a "spoke type" surface relief structures sich as shown in Fig. 6-2 should result in an array of myelinic (from the myelin sheaths of human nerves which exhibit this structure)arrangements of the smectic A phase, if the minimum interfacial tension corresponds to the molecules parallel to a flat surface. A schematic representation of 

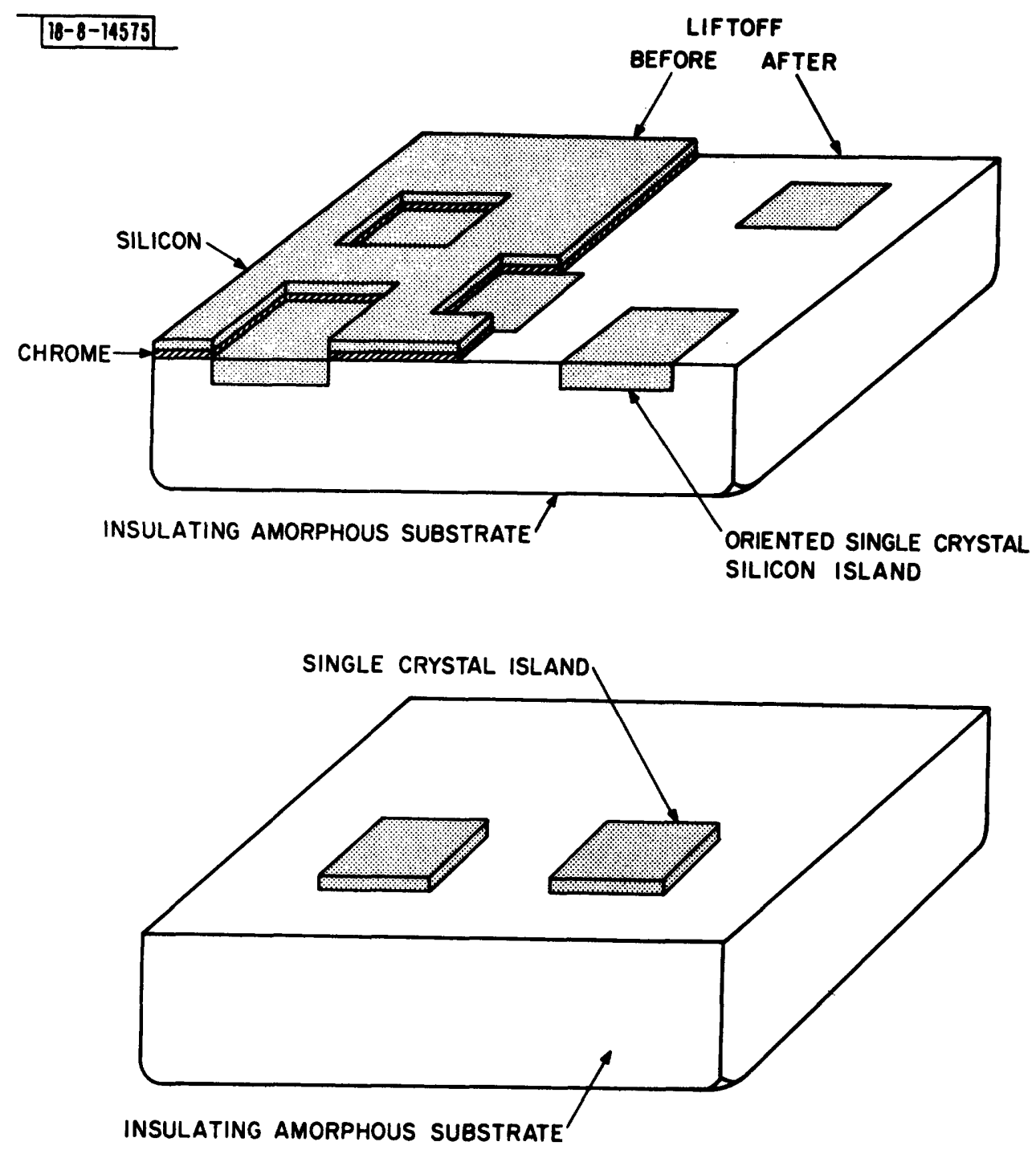

Fig. 6-1. A schematic representation of two proposed methods for producing small areas of single crystal semiconductor for integrated circuit devices. In the upper illustration the semiconductor islands are confined and oriented by a square pit in the amorphous substrate. In the lower illustration the semiconductor islands are first defined by etching a continuous $f i l m$, and are then annealed to yield single crystal islands. 


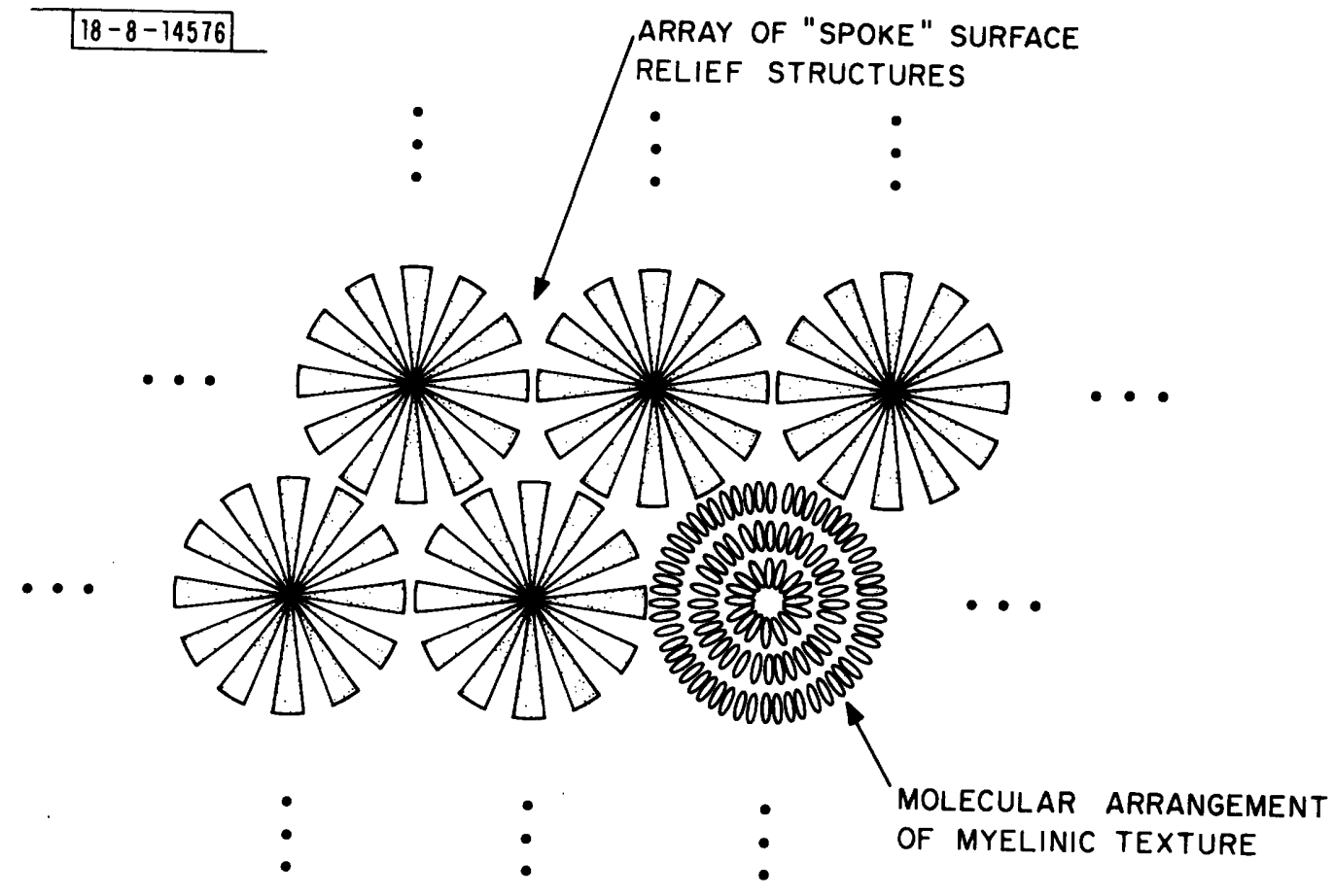

Fig. 6-2. A hexagonal-close-packed array of "spoke" surface relief structures is shown. A "sandwich" such as shown in Fig. 4-4 of two of these structures and a smectic A liquid crystal should lead to an array of "myelinic" structures. The molecular arrangement in the myelinic structure is shown schematically. The same configuration would yield an array of $360^{\circ}$ and $180^{\circ}$ wedge disclinations for a nematic liquid crystal. 
the predicted texture is also shown in Fig. 6-2. The same array of "spoke" structureswould produce an array of $360^{\circ}$ and $180^{\circ}$ wedge disclinations in a nematic material. Perhaps such manipulations of organic molecules could lead to methods for making useful molecular assemblies.

This example of inducing an array of defect structures illustrates one of the unique capabilities of the method of orienting crystalline overlayers by artificial surface-relief structures. Because the orienting surface-relief structures can be directly controlled one is not restricted to the production of uniformly oriented overlayers. It should be possible to produce overlayers with rapid variations in crystallographic direction which could be obtained in no other way.

Other ideas too numerous to be recorded here have been stimulated by this work. In essence a new degree of freedom has been introduced in the science and technology of surfaces. It is believed that the models and demonstrations of overlayer orientation presented here are but a first example of what will be an exciting new field of investigation. 


\section{APPENDIX A - ANISOTROPIC ETCHING OF SILICON}

The anisotropic etching of silicon is the key step in the preparation of $X$-ray masks with silicon nitride - silicon dioxide $\left(\mathrm{Si}_{3} \mathrm{~N}_{4}-\mathrm{SiO}_{2}\right)$ transmitter membranes. It is also the key step in the preparation of samples for viewing in transmission electron microscopes.

The anisotropic etch used is a solution of ethlylene diamine pyro83

catachol (EDP) in water. The solution is prepared by mixing in a nitrogen atmosphere 45 grams of pyrocatochol $\left(\mathrm{C}_{6} \mathrm{H}_{4}\left(\mathrm{OH}_{2}\right)\right), 255 \mathrm{ml}$ of ethylenediamine $\left(\mathrm{NH}_{2}\left(\mathrm{CH}_{2}\right)_{2} \mathrm{NH}_{2}\right)$ and $120 \mathrm{ml}$ of water. This solution etches the $\{111\}$ planes of silicon $\approx 16$ times slower than the' $\{100\}$ planes.

\section{$X$-RAY MASK FABRICATION}

The cross-section of a $\mathrm{Si}_{3} \mathrm{~N}_{4}-\mathrm{SiO}_{2} \mathrm{X}$-ray mask is shown in Fig. A-1. The mask is fabricated by first depositing films of $100 \mathrm{~nm}$ of $\mathrm{Si}_{3} \mathrm{~N}_{4}$ and $100 \mathrm{~nm}$ of $\mathrm{SiO}_{2}$ on the front side of a $\{100\}$ oriented silicon wafer. A film of $500 \mathrm{~nm}$ of $\mathrm{SiO}_{2}$ is then deposited on the back of the silicon wafer. The $\mathrm{Si}_{3} \mathrm{~N}_{4}$ and $\mathrm{SiO}_{2}$ layers are deposited by a commercial chemical vapor deposition CVD process. After the films have been deposited a "window" or a pattern of "windows" is etched into the silicon dioxide on the back of the wafer. Because large area masks are fragile, the windows should be smaller than $2 \mathrm{~mm} \times 2 \mathrm{~mm}$. The straight edges of the windows are aligned with the $\langle 110\rangle$ direction in the plane of the wafer. The silicon dioxide "window" pattern acts as a mask during anisotropic etching of the silicon. The gold absorber pattern is defined on the front side of the sample using any pattern 


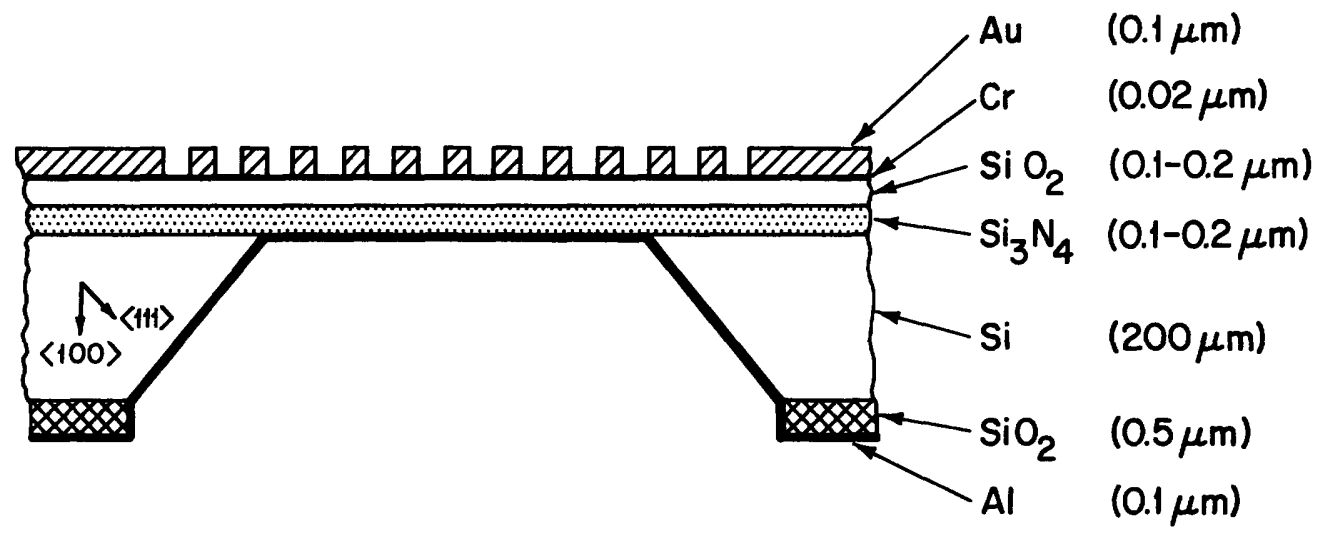

Fig. A-1. Schematic cross-section of a $\mathrm{Si}_{3} \mathrm{~N}_{4}$ membrane X-ray mask. 
fabrication method compatible with silicon processing. As a final step the silicon is anisotropically etched away beneath the absorber pattern to yield a $200 \mathrm{~nm}$ thick membrane supported on a silicon frame.

During anisotropic etching the silicon wafer is held in a special holder to protect the front side. The front must be protected because the EDP etch slowly attacks silicon dioxide. The special holder and the etching configurations are shown schematically in Fig. A-2. The holder is made from glass or fuzed quartz. It consists simply of a flat plate with a passage provided to a vent tube. The front side of the silicon wafer is held against a Buna-N rubber 0-ring by a teflon retaining ring. The cavity between the silicon and the glass holder can be filled with glycerol or it can remain empty. (If any leaks should develope around the 0-ring the glycerol serves to dilute the etchant). Glycerol is used rather than water because its boiling point is higher than the $105{ }^{\circ} \mathrm{C}$ etchant temperature required to achieve optimum etch rate. (The etch rate for EDP at $105{ }^{\circ} \mathrm{C}$ is $\approx 1 / \mu \mathrm{mi} / \mathrm{min}$. When all the silicon has been removed from the window areas etching ceases because $\mathrm{Si}_{3} \mathrm{~N}_{4}$ is not attacked by EDP. The X-ray mask is removed from the etching holder and rinsed in methanol and water. The mask is completed by evaporating a layer of aluminum on the backside. SAMPLE THINNING FOR MICROSCOPY

Samples are prepared and etched for examination by transmission electron microscopy using procedures similar to those used for X-ray mask fabrication. 


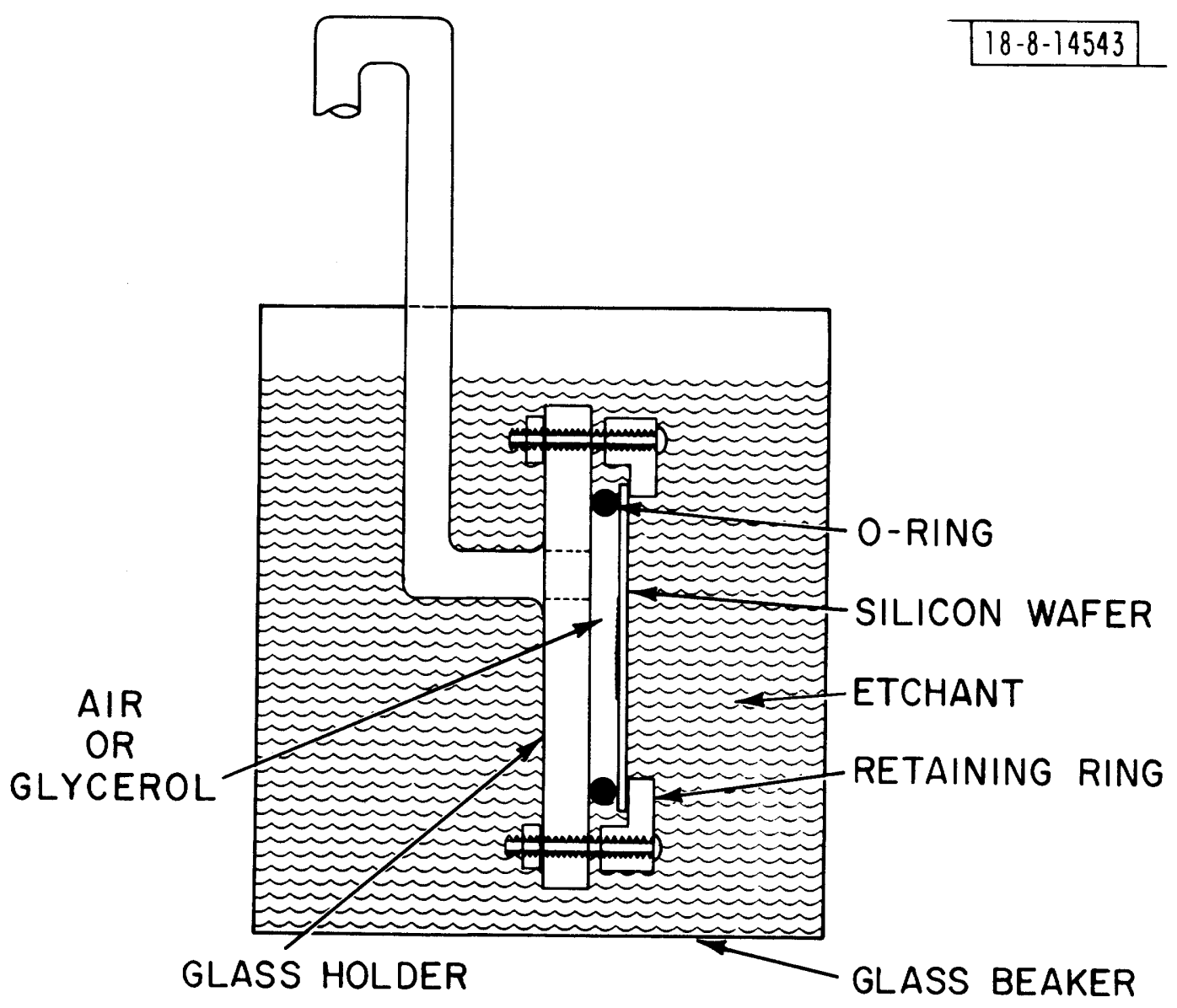

Fig. A-2 Schematic diagram of the sample holder used during anisotropic etching of silicon wafers. 
A film of CVD Si ${ }_{3} \mathrm{~N}_{4}$ and $\mathrm{SiO}_{2}$ or thermally grown $\mathrm{SiO}_{2}$ is deposited on the front side of the silicon wafer to a total thickness of less than $200 \mathrm{~nm}$. $500 \mathrm{~nm}$ of $\mathrm{SiO}_{2}$ is deposited on the backside of the wafer by CVD. A window pattern is etched into the $\mathrm{SiO}_{2}$ on the backside of the wafer. In this case the open areas in the center of the windows are $1 \mathrm{~mm} ; x 1 \mathrm{~mm}$. Outside the open area there is another open area which defines a "frame" for the window. The outside dimensions of the frame are $2 \mathrm{~mm} \times 2 \mathrm{~mm}$. After the windows have been etched into the $\mathrm{SiO}_{2}$ on the back of the wafer a surfacerelief structure is defined on the front side of the wafer and a deposition experiment is performed. The silicon is then anisotropically etched away from the windows using EDP. During etching the front: side is protected in the same holder which is used for X-ray mask fabrication. If $\mathrm{SiO}_{2}$ alone is used on the front side the etching must be watched carefully and terminated as soon as all of the silicon has been removed because the $\mathrm{SiO}_{2}$ is attacked by EDP. Since the window pattern incorporates a "frame" the small windows can be removed from the larger (1.5" diameter) silicon wafer by breaking the thin membrane outside the frame. The resulting $2 \mathrm{~mm} \times 2 \mathrm{~mm}$ substrates are thus square silicon frames supporting a $<200 \mathrm{~nm}$ thick $1 \mathrm{~mm} x$ $1 \mathrm{~mm}$ membrane. This sample size is directly compatible with most electron microscope sample holders which are usually limited to a maximum sample size of $3 \mathrm{~mm}$ diameter. 


\section{APPENDIX B POLYIMIDE X-RAY MASK FABRICATION PROCEDURE}

The fabrication of polyimide X-ray masks can be divided into four major tasks.

B. 1 Preparation of a polyimide film on a glass substrate.

B.2 Definition of a gold absorber pattern on the polyimide film.

B.3 Removal of the glass substrate.

B.4 Mounting of the $x$-ray mask on a ring holder. 


\section{B. 1 PREPARATION OF A POLYIMIDE FILM ON A GLASS SUBSTRATE}

Any smooth glass substrate which can be completely dissolved in hydroflouric acid is satisfactory as a substrate. Corning 0211 glass $2 " \times 2 "(6-8$ mils. thick) was used for the masks fabricated for the thesis work. Dupont Product PI-2530, a polyimide plastic precursor, is used in the fabrication process. The properties of PI-2530 are discussed at the end of this appendix. The process steps for forming a polyimide film on a glass substrate are as follows:

1. The glass substrates must be free of all organic films and particulate contamination. An effective cleaning procedure for 0211 glass is:

a. Immerse for $10 \mathrm{~min}$. in trichlorethylene.

b. Immerse for $10 \mathrm{~min}$. in acetone.

c. Immerse for $10 \mathrm{~min}$. in methanol.

d. Rinse in dionized (DI) water.

e. Immerse in concentrated sulfuric acid for $10 \mathrm{~min}$.

f. Rinse in DI water.

g. Immerse in semiconductor grade detergent in water with ultrasonic agitation for $10 \mathrm{~min}$.

h. Rinse in DI water.

i. Immerse in hot flowing particulate free DI water for 60 min. 
2. The substrate surface should be examined to confirm that it is free of particles and organic residue.

3. The glass must be thoroughly dry. It can be dried using an infrared lamp or in a clean oven.

4. The glass substrate is coated with a film of polyimide precursor by spinning a diluted solution of PI-2530. A solution of 4 parts of PI-2530, 1 part N-Methyl - 2 - Pyrollidone, and 1 part acetone is applied to the substrate through a $0.2 \mu \mathrm{m}$ sintered silver filter. The substrate is spun for $120 \mathrm{sec}$. or until the film is dry. Spin speeds of $3 \mathrm{krpm}$ and $8 \mathrm{krpm} . y i e l d$ final polyimide film thicknesses of $1.4 \mu \mathrm{m}$ and $0.9 \mu \mathrm{m}$ respectively. A vented spinner must be used because the solvents are toxic. 5. The substrate is baked at $150^{\circ} \mathrm{C}$ for $15 \mathrm{~min}$. in a clean vented oven to thoroughly dry the film.

6. The film of polyimide precursor is polymerized to yield polyimide plastic by curing at $250^{\circ} \mathrm{C}$ for $60 \mathrm{~min}$. in a clean vented oven. Thus prepared, these substrates can be stored indefinitely while awaiting further processing. 


\section{B.2 DEFINITION OF A GOLD ABSORBER PATTERN ON THE POLYIMIDE FILM}

Polyimide is not seriously degraded by heating to temperatures as high as $400{ }^{\circ} \mathrm{C}$ or by the chemicals used in most microfabrication processes. It is compatible with most pattern definition techniques. The grating patterns used for the thesis work were defined by holographic lithography and ion-beam etching. The process steps are as follows:

1. To insure good adhesion between the polyimide and gold overlayer the polyimide coated glass substrates are cleaned in an oxygen plasma. About $15 \mathrm{~nm}$ of polyimide is removed by subjecting the substrates to a $30 \mathrm{sec}$. clean at 300 watts of input power using an LFE plasma asher.

2. Immediately after cleaning in the plasma asher $10 \mathrm{~nm}$ of chromium, followed by $100 \mathrm{~nm}$ of gold are deposited on the polyimide by electron beam evaporation in a high vacuum.

3. The gold is coated with $100 \mathrm{~nm}$ of Shipley AZ-1350J photoresist. A solution of 7 parts of AZ-thinner and 2 parts of AZ-1350J spun at $5 \mathrm{k}$ rpm for $30 \mathrm{sec}$. yields a film $\approx 100 \mathrm{~nm}$ thick.

4. The sample is baked at $80^{\circ} \mathrm{C}$ to drive off the photoresist solvents.

5. The photoresist outside a $1.25 \mathrm{~cm} \times 1.25 \mathrm{~cm}$ square area in the center of the sample is exposed to ultraviolet light and developed in a solution of 1 part AZ-developer to 1 part water. This leaves a square of unexposed resist in the center of the substrate.

6. A grating is exposed and developed in the remaining photoresist square using the holographic lithography process described in Section 3.2. 
7. The substrates are mounted on a water cooled flat aluminum plate which acts as a heat sink during ion-beam etching. The aluminum plate is heated to $\approx 80^{\circ} \mathrm{C}$ to liquify the Apiezon-N vacuum grease which is used to provide thermal contact between the plate and the substrate.

8. The samples are ion-beam etched using a neutralized $500 \mathrm{ev}$ argon ion beam with a current density of $0.65 \mathrm{ma} / \mathrm{cm}^{2}$. Etching is terminated when all of the gold has been removed outside the grating area. ( $\approx 90 \mathrm{sec}$ for $100 \mathrm{~nm}$ of gold.)

9. The substrates are removed from the aluminum plate by heating the plate to liquify the grease. The grease is cleaned from the substrate using trichlorethylene. 


\section{B.3 REMOVAL OF THE GLASS SUBSTRATE}

1. A mixture of Devcon 5 minute epoxy is prepared.

2. A uniform layer of the epoxy is applied to the end of a 1.5 " diameter copper tube etching holder. (See Fig. 3-12 (a).)

3. The epoxy coated copper holder is pressed to the polyimide side of the substrate and allowed to cure for $\approx 2 \mathrm{hrs}$ at room temperature.

4. The substrate and end of the holder are immersed in a solution of 2 parts Conc. HF and 1 part $\mathrm{H}_{2} \mathrm{O} .8 \mathrm{mils}$ of glass are etched in $\approx 16 \mathrm{~min}$. (See Fig. $3-12$ (b).)

5. When the glass has been completely etched away, the polyimide membrane and end of the holder are dipped into $\mathrm{H}_{2} \mathrm{O}$ and then into isopropyl alcohol. (Water will "bead up" on polyimide while it will wet glass, thus it is possible to monitor the presence of glass on the membranes by the wetting of the membrane.)

6. After a final rinse in isopropyl alcohol the polyimide membrane is blown dry with nitrogen. 


\section{B.4 MOUNTING OF THE X-RAY MASK ON A RING HOLDER}

A flat ring can be epoxied to the polyimide side of the substrate before the glass is etched away as shown in Fig. 3-12 (a). This would be done at step B.3-4. If a ring was not bonded to the polyimide at that point a support ring must be bonded after the glass has been etched away. Aluminum rings with $1 " 0 . D$. and $0.75 "$ I.D. and $0.1 "$ thickness were used for the masks fabricated for the thesis work. One surface of the ring was beveled at an angle of $5^{\circ}$. The membrane is mounted on the ring by coating the beveled surface of the ring with a low-viscosity hightemperature epoxy (Tracon 2115) 35 and gently pressing the ring to the polyimide membrane which is still attached to the copper tube etching holder. After the epoxy has cured at room temperature the superfluous membrane outside the aluminum ring is cut away with a knife.

An aluminum film for use in the electrostatic hold down scheme described in section 3.3.5 can be evaporated onto either side of the $x$-ray mask. The resulting mask configurations are shown in Figures 3-13 and $3-14$. 
PI-2530 is a polyimide precursor which consists of a solution of polyamic acid in N-Methyl - 2 - Pyrollidone and cellusolve. Upon heating, the polyamic acid polymerizes to form polyimide plastic. Polyamic acid is soluble in a very limited number of solvents. It is not soluble in any of the common solvents such as acetone, trichlorethylene or methanol. Water reacts with Polyamic acid to form a white resin which is completely insoluble in any solvent. PI-2530 has a very limited shelf life at room temperature and must be stored at a temperature of $<40{ }^{\circ} \mathrm{F}$ at all times. Because of these properties, the following precautions must be observed:

1. Water reacts with PI-2530 to form an intractable residue. Anything which touches the solution must be strictly dry.

2. Solutions of PI-2530 should be allowed to warm to room temperature in a closed container before use to avoid condensation of water on the surface of the liquid, also cold solutions of PI-2530 are very viscous and are difficult to force through $0.2 \mu$ filters.

3. Use only sintered silver filters for filtering the solution because of the corrosive nature of the PI-2530 solvents. 4. Always use a vented spinner when spinning PI-2530. The fumes are toxic.

5. Always use a vented oven for baking the PI-2530 coated substrates. Poisonous amine fumes are produced during curing. 
6. When preparing solutions of PI-2530 and 1:1 Acetone: NMP, use only fresh acetone since acetone absorbs water when exposed to air.

7. PI-2530 is very difficult to clean up and its residue can easily contaminate glassware. Discard any beakers or other containers which become coated with the solution. NMP will remove uncured PI-2530 but it is very expensive $\approx \$ 50.00 / 1$ iter. 
APPENDIX C - PREPARATION AND SPINNING OF POLYMETHLY METHACRYLATE (PMMA)

PMMA of 950,000 molecular weight was obtained from Esschem Corporation. 84 To insure that the unexposed PMMA had the minimum solubility in the developer of $60 \%$ isopropyl alcohol and $40 \%$ methyl isobutyl ketone, the 950,000 molecular weight product is "leached" in the developer. Ten grams of PMMA is mixed with $1000 \mathrm{cc}$ of developer and stirred for 24 hours, at which time the remaining PMMA is separated from the developer by vacuum filtration with a fritted glass filter. Any remaining solvent is allowed to evaporate. The "leached" PMMA is then dissolved in monochlorobenzene $\mathrm{C}_{6} \mathrm{H}_{5} \mathrm{Cl}$. Concentrations as great as $16 \%$ by weight are possible at room temperature.

Spin coating samples with solutions of PMMA in chlorobenzene requires some special precautions. The solution must be filtered to remove any suspended particles. Sintered silver filters 85 wich filter to $0.2 \mu \mathrm{m}$ have been found to be satisfactory. A vented spinner should be used because the monochlorobenzene is a toxic substance, and a moderate air velocity normal to the sample surface helps to prevent any PMMA "cobwebs", which may form at the edge of the sample during spinning, from falling on the substrate surface. After spinning (spin time 60 seconds) the PMMA film is baked at $180^{\circ} \mathrm{C}$ in flowing dry $\mathrm{N}_{2}$ for 30 minutes to drive off the solvent and to remove residual stress in the film.

A table is given below of measured PMMA film thickness for PMMA concentration of $4 \%$ and $8 \%$ by weight in $\mathrm{C}_{6} \mathrm{H}_{5} \mathrm{Cl}$ and spin speeds of $3 \mathrm{~K}, 5 \mathrm{~K}$, and 
$7 \mathrm{~K}$ rpm. The solutions are prepared with the following quantities of $\mathrm{C}_{6} \mathrm{H}_{5} \mathrm{Cl}$ and PMMA.

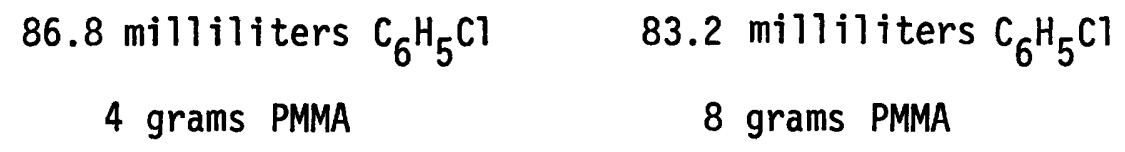

100 grams of $4 \%$ solution

\section{0 grams of $8 \%$ solution}

Table D-1 PMMA thickness versus spin speed and concentration in $\mathrm{C}_{6} \mathrm{H}_{5} \mathrm{Cl}$ by weight

\begin{tabular}{|c|c|c|c|}
\hline & $3 K_{,}, \mathrm{rpm}$ & $5 \mathrm{~K} . \mathrm{rpm}$ & $7 \mathrm{~K} \mathrm{rpm}$ \\
\hline $\begin{array}{c}4 \% \\
\text { Solution }\end{array}$ & $330 \mathrm{~nm}$ & $240 \mathrm{~nm}$ & $210 \mathrm{~nm}$ \\
\hline $\begin{array}{c}8 \% \\
\text { Solution }\end{array}$ & $1250 \mathrm{~nm}$ & $1000 \mathrm{~nm}$ & $860 \mathrm{~nm}$ \\
\hline
\end{tabular}




\section{APPENDIX D PROPERTIES OF MBBA AND M-24}

\section{1 MBBA}

MBBA is an abreviation for $N$-Cp-methoxybenzylidene -p-butylaniline with the formula:

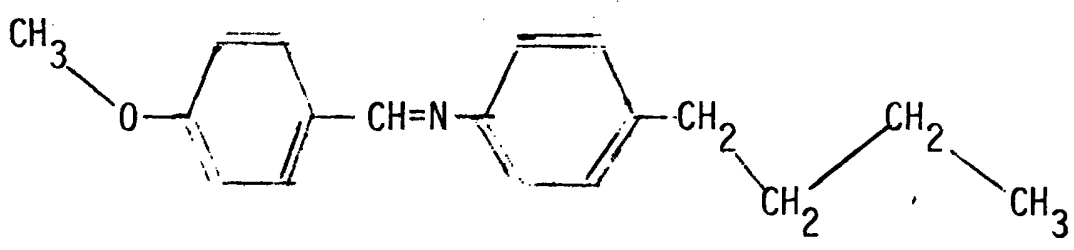

MBBA is a "thermotropic" liquid crystal which changes its phase as the temperature varies. It exhibits a nematic phase from $20{ }^{\circ} \mathrm{C}$ to $47{ }^{\circ} \mathrm{C}$. Above $47{ }^{\circ} \mathrm{C}$ MBBA is a conventional isotropic liquid.

The elastic constants which characterize MBBA are as follows:

$$
\begin{aligned}
& \text { (Splay) } k_{1}=5.3 \pm 0.5 \times 10^{-7} \text { dynes } \\
& \text { (Twist) } K_{2}=2.2 \pm 0.7 \times 10^{-7} \text { dynes } \\
& \text { (Bend) } \quad K_{3}=7.45 \pm 1.1 \times 10^{-7} \text { dynes }
\end{aligned}
$$

The indices of refraction are as follows:

$$
\begin{aligned}
& 1.5443 \text { ordinary } \\
& 1.7582 \text { extraordinary }
\end{aligned}
$$

\section{D. $2 \quad M-24$}

M-24 is an abbreviation for 4-cyano-4 ' $n$-octoxy biphenyl. M-24 
is a thermotropic liquid crystal. It exhibits isotropic, nematic, smectic $A$, and crystalline phases over the following temperature ranges:

$$
\begin{aligned}
& \text { Isotropic }-\mathrm{T} \text { above } 78.3{ }^{\circ} \mathrm{C} \\
& \text { Nematic }-66.5{ }^{\circ} \mathrm{C}<\mathrm{T}<78.3{ }^{\circ} \mathrm{C} \\
& \text { Smectic A }-54.5{ }^{\circ} \mathrm{C}<\mathrm{T}<66.5{ }^{\circ} \mathrm{C} \\
& \text { Crystalline }-\mathrm{T}<54.5{ }^{\circ} \mathrm{C}
\end{aligned}
$$




\section{REFERENCES}

1. Maissel and Glang, Handbook of Thin Film Technology, New York: McGraw Hi11, 1970.

2. K. L. Chopra, Thin Film Phenomena, New York: McGraw Hill, 1969.

3. E. Bauer, "Fiber Texture", Trans. of the Ninth Nat. Vac. Sym. of the A.V.S., New York: The MacMillan Co., pp. 35-44, 1962.

E. Bauer, "Growth of Oriented Films on Amorphous Surfaces", in Single Crystal Films eds. M. H. Francombe and H. Sato, London: Pergamon, pp. 43-67, 1964.

4. Henry I. Smith, "Fabrication Techniques for Surface Acoustic Wave and Thin Film Optical Devices", Proc. IEEE, Vol. 62, pp. 1361-1387 oct. 1974 .

5. P. G. deGennes, The Physics of Liquid Crystals, Oxford: Clarendon Press, 1974.

6. J. M. Blakely, Introduction to the Properties of Crystal Surfaces 0xford: Pergamon Press, 1973.

7. Conyers Herring, "Some Theorems on the Free Energies of Crystal Surfaces", Phys. Rev., Vo1. 82, pp. 87-93, Apr. 1951.

8. Conyers Herring, "The Use of Classical Macroscopic Concepts in Surface-Energy Problems", in Structure and Properties of Solid Surfaces ed. by Gomer and Smith, Chicago: University of Chicago Press, 1953, pp. 5-82.

9. R. J. Hawryluk, "Energy Dissipation by Electron Beam Scattering in Thin Polymer Films", Ph.D. dissertation, Mass. Inst. Technol., May, 1974.

10. D. Rudolph and G. Schmahl, "Verfahren sur Herstellung von Pontgenlinser and Beugungsgittern", Umschan Wissi. Tech., vol. 67, pg. 225, 1967.

11. Shipley Co.: Newton, Mass.

12. F. H. Dill, W. P. Hornberger, P.S. Hauge, and J. M. Shaw, "Characterization of Positive Photoresists", IEEE Trans. Elec. Dev., Vol. ED-22, pp. 445-452, July, 1975.

13. W. T. Tsang and S. Wang, "Simultaneous Exposure and Development Technique for Making Gratings on Positive Photoresist", Appl. Phys. Lett., vol. 24, p. 519, 1974. 
14. W. T. Tsang, "Theoretical Modeling of the Simultaneous Exposure and Development (SED) process of a Positive Photoresist", App1. Opt., vol. 16, pp. 1918-1930.

15. R. A. Harris, "Polymethyl Methacrylate as an Electron Sensitive Resist", J. Electrochem. Soc., vol. 120 pp. 270-274, Feb. 1973.

16. E. Spiller, R. Feder, J. Topalian, D. Eastman, W. Gudat and D. Sayre, "X-ray Microscopy of Biological Objects with Carbon $K$ and with Synchrotron Radiation", IBM Research Report, RC 5675 ( $\left.{ }^{\alpha} 24437\right)$ Physics (General), Oct. 1975.

17. H. Hiraoka, "Radiation Chemistry of Poly(methacrylates)", IBM J. Res. Dev., vol. 21, pp. 121-131, March 1977. (See the same issue for other papers.)

18. Stephen E. Bernacki, and Henry I. Smith, "X-ray Lithography Applied to Silicon Device Fabrication", Proceedings Sixth International Conference on Electron and Ion Beam Science and Technology, R. Bakish, ed., Princeton, N. J.: The Electrochemical Society, 1974, pp. 34-46.

19. C. A. Andersen, in The Electron Mircoprobe, T. P. McKinley, K. F. J. Heinrich, and D. B. Wittry, Eds., New York: Wiley, 1966, pg. 58.

20. R. Feder, E. Spiller, and J. Topalian, "Replication of $0.1 \mu \mathrm{m}$ Geometries with X-ray Lithography", J. Vac. Sci. Technol., vol. 12, pp. 1332-1335, Nova/Dec. 1975.

21. B. L. Henke and E. S. Ebisu, "Low Energy X-ray and Electron Absorption in Solids," Advances in X-ray Analysis, New York: Plenum Press, vol. 17, p. 150, 1974.

22. J. B. Woodhouse, A. L. A. Fields, and I. A. Bucklow, "X-ray Mass Absorption Coefficients for Gold, Lead, and Bismuth in the Range 1-10 A," J. Phys. D. Appl. Phys. vol. 7, pp. 483-489, 1974.

23. D. C. Flanders and Henry I. Smith, "Surface Relief Gratings of 3200 A Period-Fabrication and Influence on Thin Film Growth," J. Vac. Sci. Technol. vol. 15, March/April 1978.

24. Vacuum Generators Limited; Sussex, England.

25. H. I. Smith, "X-ray Lithography: A complementary Technique to Electron Beam Lithography," J. Vac. Sci. Technol., vol. 10, pp. 913-917, Nov./Dec. 1973. 
26. D. L. Spears, H. I. Smith, and E. Stern, "X-ray Replication of Scanning Electron Microscope Generated Patterns," in Proc. 5th Int. Conf. on Electron and Ion Beam Science and Technology, R. Bakish, Ed. (Houston, Tex., May 7-11, 1972) published by The Electrochem. Soc. 1972.

27. D. Maydan, G. H. Coquin, V. R. Maldonado, S. Somekh, D. Y. Lou, and G. N. Taylor, "High Speed Replication of Submicron Features on Large Areas by X-ray Lithography," IEEE Trans. Electron Devices, vol. ED-22 pg. 429 (1975).

28. J. S. Greeneich, "X-ray Lithography: Part I - Design Criteria for Optimizing Resist Energy Absorption; Part II - Pattern Replication with Polymer Masks," IEEE Trans. Electron Devices vol. ED-22, pg. 434 (1975).

29. E. Bassous, R. Feder, E. Spiller, and J. Topalian, "High Transmission X-ray Masks for Lithographic Applications," Solid State Technol., pp. 55-58, Sept. 1976.

30. Henry I. Smith and D. C. Flanders, "X-ray Lithography," Proc. 18th Conf. on Solid State Dev., Tokyo 1976, Jap. J. App1. Phys., vol. 16, Sup. 16-1, pp. 67-65, 1977.

31. D. C. Flanders, and Henry I. Smith, "Polyimide Membrane X-ray Lithography Mask -Fabrication and Distortion Measurements," J. Vac. Sci. Technol., vol. 15, March/April 1978.

32. E. I. DuPont DeNemours and Co., Fabrics and Finishes Dept., Wilmington, Delaware 19898.

33. Corning Glass Works, Danville, Virginia.

34. Devcon 5 min. epoxy, Devcon Corp., Danvers, Mass.

35. Tra-Con 2115 epoxy, Tra-Con, Medford, Mass.

36. H. I. Smith, "Ion Beam Etching," Proc. Symp. on Etching for Pattern Definition, Eds. H. G. Hughes, M. J. Rand, The Electrochemical Society, 1976.

37. H. W. Lehmann and R. Widmer, "Profile Control by Reactive Sputter Etching," J. Vac. Sci. and Technol. Jan./Feb. 1978.

38. H. W. Lehmann and R. Widmer, "Fabrication of Deep Square Wave Structures with Micron Dimensions by Reactive Sputter Etching," App1. Phys. Lett. vol. 32, Feb. 1978. 
39. D. C. Flanders, H. I. Smith, H. W. Lehmann, R. Widmer and D. C.oShaver, "Surface Relief Structures with Linewidths below 2000 A," Appi. Phys. Lett., vol. 32, pp. 112-114, Jan. (1978).

40. H. W. Lehmann, L. Krausbauer, and R. Widmer, "Redeposition - A Serious Problem in Rf-Sputter Etching of Structures with Micron Dimensions," J. Vac. Sci. Technol., vol. 14, 281-284 (1977).

41. P. Gloersen, "Masking for Ion-Beam Etching," Solid State Technol.; April 1976.

42. K. Knop, "Color Pictures Using the Zero Diffraction Order of Phase Grating Structures," Optics Comm., vol. 18, p. 298, 1976.

43. J. W. Coburn, H. F. Winters, and T. J. Chuang, "Ion-Surface Interactions in Plasma Etching," J. Appl. Phys., vol. 48, pp. 3532-3540, Aug. 1977.

44. John R. Vig., "U.V/Ozone Cleaning of Surfaces," IEEE Trans. Pts., hyb., Pack., vol. PHP-12, pp. 365-370, Dec. 1976.

45. Model R-52; Ultra-Violet Products Inc., San Gabriel, Calif.

46. E. B. Priestley, Peter J. Wajtowicz, and Ping Sheng, Introduction to Liquid Crystals, New York: Plenum Press, 1975, pp. 222-233.

47. Ralph Schaetzing, M.I.T. Material Science Center.

48. Frederic J. Kahn, Gary N. Taylor, and Harold Schonhorn, "Surface-Produced Alignment of Liquid Crystals," Proc. IEEE vol. 61, pp. 823-828, July 1973.

49. J. F. Dryer, "Epitaxy of Nematic Liquid Crystals," in Liquid Crystals 3 , Eds. C. H. Brown, and M. Labes, London: Gordon and Breach, $1973, \mathrm{pg} .1113$.

50. L. T. Creagh and A. R. Kmetz, "Mechanism of Surface Alignment in Nematic Liquid Crystals," Mo1. Cryst. and Liq. Cryst. vol. 24, pg. 59, 1973.

51. William F. Harris, "Disclinations," Scientific American, vo1. 237, pp. 130-145, Dec. 1977.

52. F. Grandjean, Bu11. Soc. Fr. Miner., vol. 29, p. 164 (1916).

53. W. Urbach, M. Boix, and E. Guyon, "Alignment of Nematics and Smectics on Evaporated Films," Appl. Phys. Lett., vol. 25, pp. 479-481, Nov. 1974. 
54. G. D. Dixon, T. P. Brody, and W. A. Hester, "Alignment Mechanism in Twisted Nematic Layers," Appl. Phys. Lett., vol. 24, p. 47, 1974 .

55. J. E. Proust, L. Ter-Minassian-Saraga, and E. Gayon, "Orientation of a Nematic Liquid Crystal by Suitable Boundary Conditions," Sol. St. Commun., vol. 11, p. 1227, 1972.

56. Dwight W. Berreman, "Solid Surface Shape and the Alignment on an Adjacent Nematic Liquid Crystal," Phys. Rev. Lett., vol. 28, pp. 1682-1686, June 1972.

57. Lawrence Goodman, J. T. McGinn, Charles H. Anderson, and Frank Digeronimo, "Topography of Obliquely Evaporated Silicon 0xide Films and its Effect on Liquid Crystal Orientation," IEEE Trans. Electron Devices, vol. ED-24, pp. 795-804, July 1977.

58. P. Chatelain, Bu11. Soc. Franc. Miner. Christ., vol. 66, p. 105, 1943.

59. Opto-Sil 2, Amersil Inc., Hillside, N. J.

60. D. C. Flanders and Henry I. Smith, "A New Interferometric Alignment Technique," Appl. Phys. Lett., vol. 31, pp. 426-428, 0ct. 1977.

61. J. F. Nye, Physical Properties of Crystals, Oxford: Clarendon Press, 1957, pg. 235.

62. DODCI - Kodak; Rochester, N. Y. - This technique of determining the orientation of the nematic director was suggested by David Shaver at M.I.T. Lincoln Laboratory.

63. J. W. Matthews, Epitaxial Growth, New York: Academic Press, 1975.

64. G. A. Bassett, "A New Technique for Decoration of Cleavage and Slip Steps on Ionic Crystal Surfaces," Phil. Mag., vol. 3, p. 1042, 1958.

65. D. W. Pashley, M. J. Stowe11, M. H. Jacobs, and T. J. Law, "The Growth and Structure of Gold and Silver Deposits Formed by Evaporation Inside an Electron Microscope," Phil. Mag., vol. 10, pp. 127-158, 1964.

66. G. I. Distler, V. P. Vlasov and V. M. Kanevsky, "Orientational and Long Range Effects in Epitaxy," Thin Solid Films, vol. 33, pp. 287-300, 1976. (For a critique of this work see: K. L. Chopra, 
"Epitaxial Growth of Films on Substrates Coated with Amorphous Deposits," J. Appl. Phys., vol. 40, pp. 906-907, 1969.)

67. G. I. Distler, "Epitaxy as a Matrix Replicating Process," Thin Solid Films, vol. 32, pp. 157-162, 1976.

68. D. L. Rode, W. R. Wagner, and N. E. Schumaker, "Singular Instabilities on LPE GaAs, CVD Si, and MBE InP Growth Surfaces," App1. Phys. Lett., vol. 30, pp. 75-78, Jan. 1977.

69. W. L. Winterbottom. "Equilibrium Shape of a Small Particle in Contact with a Foreign Substrate," Acta. Met., vol. 15, pp. 303310, Feb. 1967.

70. Landau and Lifshitz, Statistical Physics, New York: Pergamon, pp. 460-463 1969.

71. David Robertson and G. M. Pound, "Heterogenous Nucleation and Film Growth," CRC Critical Reviews in Solid State Science, pp. 163-204, Feb. 1974.

72. J. L. Kenty and J. P. Hirth, "Epitaxy and Heterogeneous Nucleation Theory," Surface Sci., vo1. 15, pp. 403-424, 1969.

73. B. K. Chakraverty and G. M. Pound, "Heterogeneous Nucleation at Macroscopic Steps," Acta. Met. vol. 12, p. 851, 1961.

74. G. R. Hennig, "Decoration of Graphite Surfaces for Electron Microscopy," Appl. Phys. Lett. vol. 4, p. 52, 1964.

75. K. L. Chopra, "Growth of Sputtered-vs-Evaporated Films," J. Appl. Phys. vol. 9, p. 3405, 1966.

76. G. Shinaoka and G. Komoriya, "The Structure of Tin Thin Films Evaporated Onto a Clean $\mathrm{NaCl}$ Substrate," J. Vac. Sci. and Technol., vo1. 7, pp. 178-181, 1970.

77. M. Adamov, B. Perovic, and T. Nenadovic, "Electrical and Structural Properties of Thin Gold Films Obtained by Vacuum Evaporation and Sputtering," Thin Solid Films, vol. 24, pp. 89-100, 1974.

78. J. B. Preece, H. Wilman, and C. T. H. Stoddart, "The Effect of Low-Pressure 0xygen, Present During Condensation, on the Structure of Tin Films," Phil. Mag., vol. 16, pp. 447-457, 1967.

79. J. D. Filby and S. Nielsen, "Progress Toward Single Crystal Silicon Films on Amorphous Substrates," J. Electro. Chem. Soc., vol. pp. 957- 58, Sept. 1965. 
80. L. G. Schu1z, Acta Cryst. vol. 4, p. 483 (1951).

81. A. N. Broers et al, "Electron Beam Fabrication of $80 \AA$ Metal Structure," Appl. Phys. Lett. vol. 29, p. 596, 1976.

82. H. P. Zingsheim, "STEM as a Tool in the Construction of TwoDimensional Molecular Assemblies," Proc. of the 10th Ann. S.E.M. Symposium, 1977.

83. R. M. Finne and D. L. Klein, "A Water-Amine Complexing Agent System for Etching Silicon," J. Electrochem. Soc., vol. 114, pp. 965-970, Sept. 1967.

84. Esschem Corp., Essington, Pa.

85. Flotronics, Selas Flotronics, Spring House, Pa.

86. BDH 